University of Tennessee Health Science Center UTHSC Digital Commons

$12-2018$

\title{
Effect of Comorbidities on HIV-1 Pathogenesis in Monocytic Cells
}

Sabina Ranjit

Follow this and additional works at: https://dc.uthsc.edu/dissertations

\section{Recommended Citation}

Ranjit, Sabina (http://orcid.org/0000-0001-5607-8403), "Effect of Comorbidities on HIV-1 Pathogenesis in Monocytic Cells" (2018). Theses and Dissertations (ETD). Paper 472. http://dx.doi.org/10.21007/ etd.cghs.2018.0467.

This Dissertation is brought to you for free and open access by the College of Graduate Health Sciences at UTHSC Digital Commons. It has been accepted for inclusion in Theses and Dissertations (ETD) by an authorized administrator of UTHSC Digital Commons. For more information, please contact jwelch30@uthsc.edu. 


\title{
Effect of Comorbidities on HIV-1 Pathogenesis in Monocytic Cells
}

\begin{abstract}
The advent of antiretroviral therapy (ART) has substantially increased the life span of people living with HIV-1 (PLWH). However, the presence of comorbid conditions such as high prevalence of drug abuse and coinfections have deteriorated the health outcomes in PLWH and progression of HIV-1 infection to AIDS. In this study, we will be focusing on the independent effect of two major comorbidities: cigarette smoking and human papilloma virus (HPV) coinfection on HIV-1 replication and pathogenesis in monocytic cells.
\end{abstract}

The prevalence of smoking is approximately 3-times higher in PLWH compared to the general population. Scientific evidences suggest that smoking aggravates HIV-1 pathogenesis and leads to decreased responses to ART. However, the exact mechanism by which smoking enhances HIV-1 replication is still not clear. The Kumar group has demonstrated the role of nicotine, at least in part, on HIV-1 replication in monocytic cells via cytochrome P450 (CYP)-mediated oxidative stress pathway. They have also shown the role of cigarette smoke condensate (CSC), which contains numerous organic compounds such as polyaryl hydrocarbons (PAHs), on cytotoxicity and HIV-1 replication in monocytic cells. Therefore, the goal in this study was to identify active ingredient of CSC, especially PAHs, which is responsible for cytotoxicity and HIV-1 replication in monocytic cells, and their underlying mechanism. PAHs such as benzo(a)pyrene $(\mathrm{BaP})$, naphthalene (NPh), phenanthrene (Phe), benzo(a)antharacene (BeA), and benzo(b)fluoranthene (BeF) are known carcinogens present in cigarette smoke. As a first part of our study, we examined the relative effect of these constituents on the cytotoxicity of monocytic cells and the possible mechanism of PAH-mediated cytotoxicity. We examined the acute (6-24 hours) and chronic (7 days) effects of these PAHs on the expression of cytochromes P450 (CYPs), oxidative stress, and cytotoxicity. The treated cells were examined for mRNA and protein levels of CYPs (1A1 and 3A4) and antioxidants enzymes (AOEs) superoxide dismutase-1 (SOD1) and catalase. Further, we assessed the levels of reactive oxygen species (ROS), caspase-3 cleavage activity, and cell viability. We performed these experiments in U937 cell lines and/or primary monocytic cells. Of the five PAHs tested, after chronic treatment only BaP (100 nM) showed a significant increase in the expression of CYP1A1, AOEs (SOD1 and catalase), ROS generation, caspase-3 cleavage activity, and cytotoxicity. However, acute treatment with BaP showed only an increase in the mRNA expression of CYP1A1. These results suggested that of the five PAHs tested, BaP is the major contributor to the toxic effect of PAHs in monocytic cells, which is likely to occur through CYP and oxidative stress pathways.

Secondly, we investigated a molecular mechanism that would explain BaPinduced HIV-1 replication. We hypothesized that CYP-mediated BaP metabolism generates ROS, and the resultant oxidative stress aggravates HIV-1 replication. As expected, we observed $~ 4$-fold increase in HIV-1 replication in differentiated U1 (HIV-1- infected U937) monocytic cell lines and human primary macrophages after chronic BaP exposure. We also observed 30-fold increase in the expression of CYP1A1 at mRNA level, 2-fold increase in its enzymatic activity, as well as, an elevated ROS level and cytotoxicity in U1 cells. The knock-down of the CYP1A1 gene using siRNA and treatment with selective CYP inhibitors and antioxidants significantly reduced HIV-1 replication. Further, we observed a nuclear translocation of NF-KB subunits ( $\mathrm{p} 50$ and p65) after chronic BaP exposure, which was reduced by treatment with siRNA/ inhibitors of CYP and antioxidants. Suppression of NF-KB pathway using specific NF-KB inhibitors also significantly reduced HIV-1 replication. Together, our results suggest that BaP enhances the HIV-1 replication in macrophages by CYP-mediated oxidative stress pathway via NF-KB signaling cascade.

Thirdly, we explored the underlying mechanism by which HPV increases HIV-1 pathogenesis. HPV infection is one of the major factors that contribute to a reduced suppression of the virus in HIV-1 patients. There is a high prevalence of comorbidity of HIV-1 and HPV (which leads to cervical cancer) among HIV-1-infected population. We proposed that exosomes secreted from HPV-infected cervical 
cancer cells exacerbate HIV-1 replication in HIV-1-infected macrophages. To test the hypothesis, we treated U1 cells (HIV-1-infected monocytic cell line) with the cell culture supernatant (CCS) obtained from caski cells (HPV-infected cervical cancer cells). We observed an $\sim 2$-fold increase in HIV-1 replication in the treated $\mathrm{U} 1$ cell. We also observed a significant increase in the expression of CYPs (CYP $1 A 1$ and 2A6) at the mRNA level. However, we did not observe any significant change in the expression of CYPs as well as antioxidants enzymes (catalase, PRDX6) at the protein level. Furthermore, we isolated exosomes from the caski cell culture supernatant and observed the presence of CYPs (1A1, 2A6), SOD1 and HPV protein HPV16 E6 in caski exosomes. The exosomes derived from caski cells (CCS-Exo) significantly reduced cytotoxicity, while it increased HIV-1 replication in U1 cells. Treatment of antioxidant such as resveratrol, CYP1A1 inhibitor (ellipticine) and CYP2A6 inhibitor (Tryptamine) including chemodietary agents such as curcumin $(20 \mu \mathrm{M})$ and curcubitacin-D $(0.1 \mu \mathrm{M})$, significantly reduced the CCS and CCS-Exo mediated HIV-1 replication in U1 cells. These results suggest the role of specific CYP-induced oxidative stress pathway in HIV-1 replication. Altogether, we demonstrated that cervical cancer cells exacerbate HIV-1 replication in monocytic cells via transferring oxidative stress factors such as CYPs and HPV oncoproteins through exosomes. We also showed that the viral replication undergoes via a CYP-mediated oxidative stress pathway and it can be reduced by treatment of chemodietary agents like curcumin and Cucurbitacin-D. The present study therefore, illustrates that comorbidities such as smoking and HPV coinfection contribute to HIV-1 replication and pathogenesis in monocytic cells via a CYP-mediated oxidative stress pathway. The study also provides scientific rationale for the development of novel therapeutic treatment for PLWHs with these comorbidities by targeting CYP and specific oxidative stress pathway.

\section{Document Type}

Dissertation

Degree Name

Doctor of Philosophy (PhD)

\section{Program}

Pharmaceutical Sciences

\section{Research Advisor}

Santosh Kumar, Ph.D.

\section{Keywords}

Comorbidities, Cytochrome P450, HIV, HPV, Oxidative Stress, Smoking 


\title{
Effect of Comorbidities on HIV-1 Pathogenesis in Monocytic Cells
}

\author{
A Dissertation \\ Presented for \\ The Graduate Studies Council \\ The University of Tennessee \\ Health Science Center
}

\author{
In Partial Fulfillment \\ Of the Requirements for the Degree \\ Doctor of Philosophy \\ From The University of Tennessee
}

By

Sabina Ranjit

December 2018 
Portions of Chapters 1 and 2 (C) 2018 by F1000 Research Ltd. Portions of Chapter 2 (C) 2018 by Informa. Chapter 4 (C) 2016 by Public Library of Science. Chapter 5 (C) 2018 by Nature Publishing Group. All other material (C) 2018 by Sabina Ranjit. All rights reserved. 


\section{DEDICATION}

I dedicate this dissertation to my beloved parents, Mr. Krishna Prasad Ranjitkar and Mrs. Sanu Maiya Ranjit, for their unconditional love and support. Thank you for believing in me and encouraging me to achieve my dreams. 


\section{ACKNOWLEDGEMENTS}

I would like to express my sincere gratitude to my mentor, Dr. Santosh Kumar for his guidance and endless support to make this project a success. The graduate training with him, has not only taught me science but also the morals and ethics to become a good human being. His teachings will always motivate me to move forward in my career and my life as a whole.

I am also grateful to my committee members, Dr. Sarka Beranova-Giorgianni, Dr. Theodore J. Cory, Dr. Subhash C. Chauhan, Dr. Anil Kumar and Dr. P. David Rogers for their time, valuable inputs and suggestions to complete my project and dissertation. I would also like to acknowledge F1000 Research Ltd, Informa, Public Library of Science and Nature Publishing Group for allowing me to reprint and modify the contents from the respective manuscripts into my dissertation.

My sincere appreciation to department of Pharmaceutical Sciences for the administrative and financial support to conduct my graduate study and project. I would also like to thank National Institutes of Health for providing grants: R01: DA047178, R21: DA042374 and R01: AA022063 to fund my project.

I also appreciate the support and help from my lab mates past and present, without whom my project as well as my graduate school journey would be incomplete. Lastly, I do not have words to express my heartfelt appreciation to my parents for their continuous support and endless motivation to become a person, I am today. I am also grateful to all my family members, friends and teachers for all the love and support. Thank you god for everything. 


\begin{abstract}
The advent of antiretroviral therapy (ART) has substantially increased the life span of people living with HIV-1 (PLWH). However, the presence of comorbid conditions such as high prevalence of drug abuse and coinfections have deteriorated the health outcomes in PLWH and progression of HIV-1 infection to AIDS. In this study, we will be focusing on the independent effect of two major comorbidities: cigarette smoking and human papilloma virus (HPV) coinfection on HIV-1 replication and pathogenesis in monocytic cells.
\end{abstract}

The prevalence of smoking is approximately 3-times higher in PLWH compared to the general population. Scientific evidences suggest that smoking aggravates HIV-1 pathogenesis and leads to decreased responses to ART. However, the exact mechanism by which smoking enhances HIV-1 replication is still not clear. The Kumar group has demonstrated the role of nicotine, at least in part, on HIV-1 replication in monocytic cells via cytochrome P450 (CYP)-mediated oxidative stress pathway. They have also shown the role of cigarette smoke condensate (CSC), which contains numerous organic compounds such as polyaryl hydrocarbons (PAHs), on cytotoxicity and HIV-1 replication in monocytic cells. Therefore, the goal in this study was to identify active ingredient of CSC, especially PAHs, which is responsible for cytotoxicity and HIV-1 replication in monocytic cells, and their underlying mechanism. PAHs such as benzo(a)pyrene (BaP), naphthalene $(\mathrm{NPh})$, phenanthrene (Phe), benzo(a)antharacene (BeA), and benzo(b)fluoranthene $(\mathrm{BeF})$ are known carcinogens present in cigarette smoke. As a first part of our study, we examined the relative effect of these constituents on the cytotoxicity of monocytic cells and the possible mechanism of PAH-mediated cytotoxicity. We examined the acute (6-24 hours) and chronic (7 days) effects of these PAHs on the expression of cytochromes P450 (CYPs), oxidative stress, and cytotoxicity. The treated cells were examined for mRNA and protein levels of CYPs (1A1 and 3A4) and antioxidants enzymes (AOEs) superoxide dismutase-1 (SOD1) and catalase. Further, we assessed the levels of reactive oxygen species (ROS), caspase-3 cleavage activity, and cell viability. We performed these experiments in U937 cell lines and/or primary monocytic cells. Of the five PAHs tested, after chronic treatment only BaP (100 nM) showed a significant increase in the expression of CYP1A1, AOEs (SOD1 and catalase), ROS generation, caspase-3 cleavage activity, and cytotoxicity. However, acute treatment with $\mathrm{BaP}$ showed only an increase in the mRNA expression of CYP1A1. These results suggested that of the five PAHs tested, $\mathrm{BaP}$ is the major contributor to the toxic effect of PAHs in monocytic cells, which is likely to occur through CYP and oxidative stress pathways.

Secondly, we investigated a molecular mechanism that would explain BaPinduced HIV-1 replication. We hypothesized that CYP-mediated BaP metabolism generates ROS, and the resultant oxidative stress aggravates HIV-1 replication. As expected, we observed $\sim$-fold increase in HIV-1 replication in differentiated U1 (HIV-1infected U937) monocytic cell lines and human primary macrophages after chronic BaP exposure. We also observed $\sim 30$-fold increase in the expression of CYP1A1 at mRNA 
level, $\sim 2$-fold increase in its enzymatic activity, as well as, an elevated ROS level and cytotoxicity in U1 cells. The knock-down of the CYP1A1 gene using siRNA and treatment with selective CYP inhibitors and antioxidants significantly reduced HIV-1 replication. Further, we observed a nuclear translocation of NF- $\mathrm{kB}$ subunits ( $\mathrm{p} 50$ and p65) after chronic BaP exposure, which was reduced by treatment with siRNA/inhibitors of CYP and antioxidants. Suppression of NF- $\mathrm{kB}$ pathway using specific NF- $\mathrm{kB}$ inhibitors also significantly reduced HIV-1 replication. Together, our results suggest that $\mathrm{BaP}$ enhances the HIV-1 replication in macrophages by CYP-mediated oxidative stress pathway via NF- $\mathrm{kB}$ signaling cascade.

Thirdly, we explored the underlying mechanism by which HPV increases HIV-1 pathogenesis. HPV infection is one of the major factors that contribute to a reduced suppression of the virus in HIV-1 patients. There is a high prevalence of comorbidity of HIV-1 and HPV (which leads to cervical cancer) among HIV-1-infected population. We proposed that exosomes secreted from HPV-infected cervical cancer cells exacerbate HIV-1 replication in HIV-1-infected macrophages. To test the hypothesis, we treated U1 cells (HIV-1-infected monocytic cell line) with the cell culture supernatant (CCS) obtained from caski cells (HPV-infected cervical cancer cells). We observed an $\sim 2$-fold increase in HIV-1 replication in the treated U1 cell. We also observed a significant increase in the expression of CYPs (CYP 1A1 and 2A6) at the mRNA level. However, we did not observe any significant change in the expression of CYPs as well as antioxidants enzymes (catalase, PRDX6) at the protein level. Furthermore, we isolated exosomes from the caski cell culture supernatant and observed the presence of CYPs (1A1, 2A6), SOD1 and HPV protein HPV16 E6 in caski exosomes. The exosomes derived from caski cells (CCS-Exo) significantly reduced cytotoxicity, while it increased HIV-1 replication in U1 cells. Treatment of antioxidant such as resveratrol, CYP1A1 inhibitor (ellipticine) and CYP2A6 inhibitor (Tryptamine) including chemodietary agents such as curcumin $(20 \mu \mathrm{M})$ and curcubitacin-D $(0.1 \mu \mathrm{M})$, significantly reduced the CCS and CCS-Exo mediated HIV-1 replication in U1 cells. These results suggest the role of specific CYP-induced oxidative stress pathway in HIV-1 replication. Altogether, we demonstrated that cervical cancer cells exacerbate HIV-1 replication in monocytic cells via transferring oxidative stress factors such as CYPs and HPV oncoproteins through exosomes. We also showed that the viral replication undergoes via a CYP-mediated oxidative stress pathway and it can be reduced by treatment of chemodietary agents like curcumin and Cucurbitacin-D.

The present study therefore, illustrates that comorbidities such as smoking and HPV coinfection contribute to HIV-1 replication and pathogenesis in monocytic cells via a CYP-mediated oxidative stress pathway. The study also provides scientific rationale for the development of novel therapeutic treatment for PLWHs with these comorbidities by targeting CYP and specific oxidative stress pathway. 


\section{TABLE OF CONTENTS}

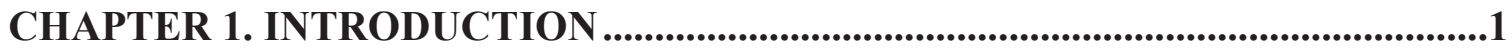

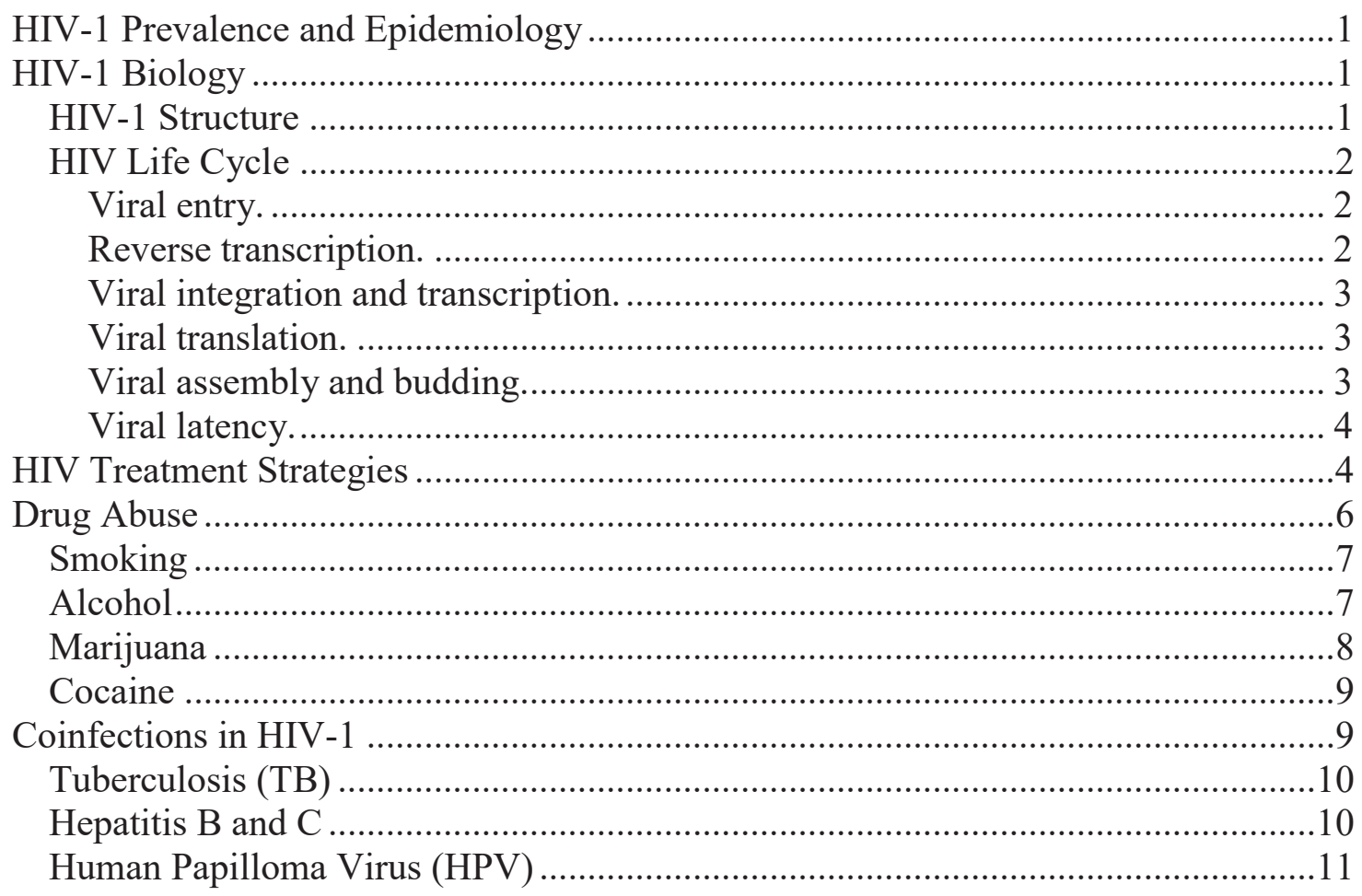

\section{CHAPTER 2. INTERACTION OF HIV-1 WITH TOBACCO SMOKING AND}

HPV INFECTION ..................................................................................................13

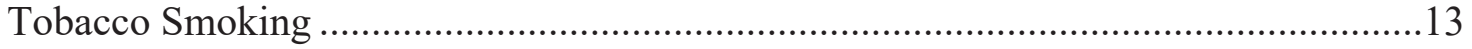

Tobacco Prevalence in HIV-1 Patients ……………………………………….....13

Effect of Tobacco on HIV-1 Pathogenesis ...............................................................13

Effect of Tobacco on HIV-1 Treatment Outcomes....................................................15

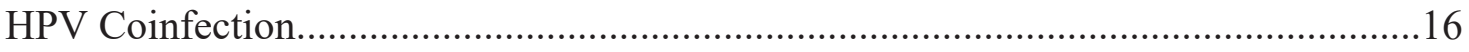

HPV Prevalence in HIV-1-infected Patients..........................................................16

Effect of HPV on HIV-1 Pathogenesis .................................................................18

Effect of Antiretroviral Therapy on HPV ………………….................................2

CHAPTER 3. SPECIFIC AIMS OF THE STUDY ......................................................22

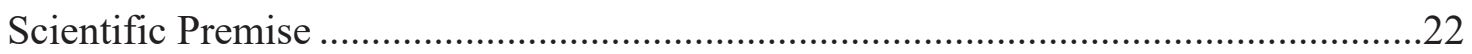

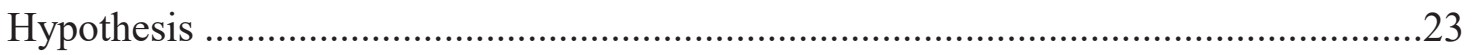

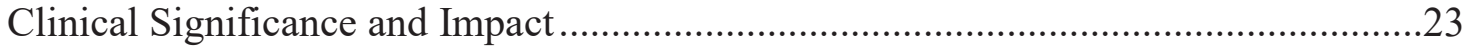

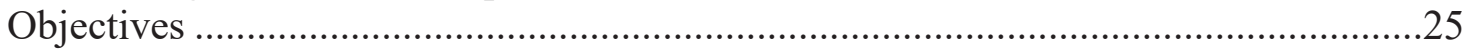

Aim 1: Study of Contribution of CYP Enzymes in PAH-enhanced Cytotoxicity

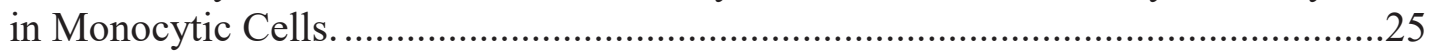

Aim 2: Study of Contribution of CYP Enzymes in BaP-enhanced HIV-1

Replication in Monocytic Cells Latently Infected with HIV-1 
Aim 3: Study of Contribution of Exosomes Derived from HPV-infected Cervical

Cancer Cells in Enhancing HIV-1 Replication in U1 cells. .26

\section{CHAPTER 4. EFFECT OF POLYARYL HYDROCARBONS ON} CYTOTOXICITY IN MONOCYTIC CELLS .....................................................................27

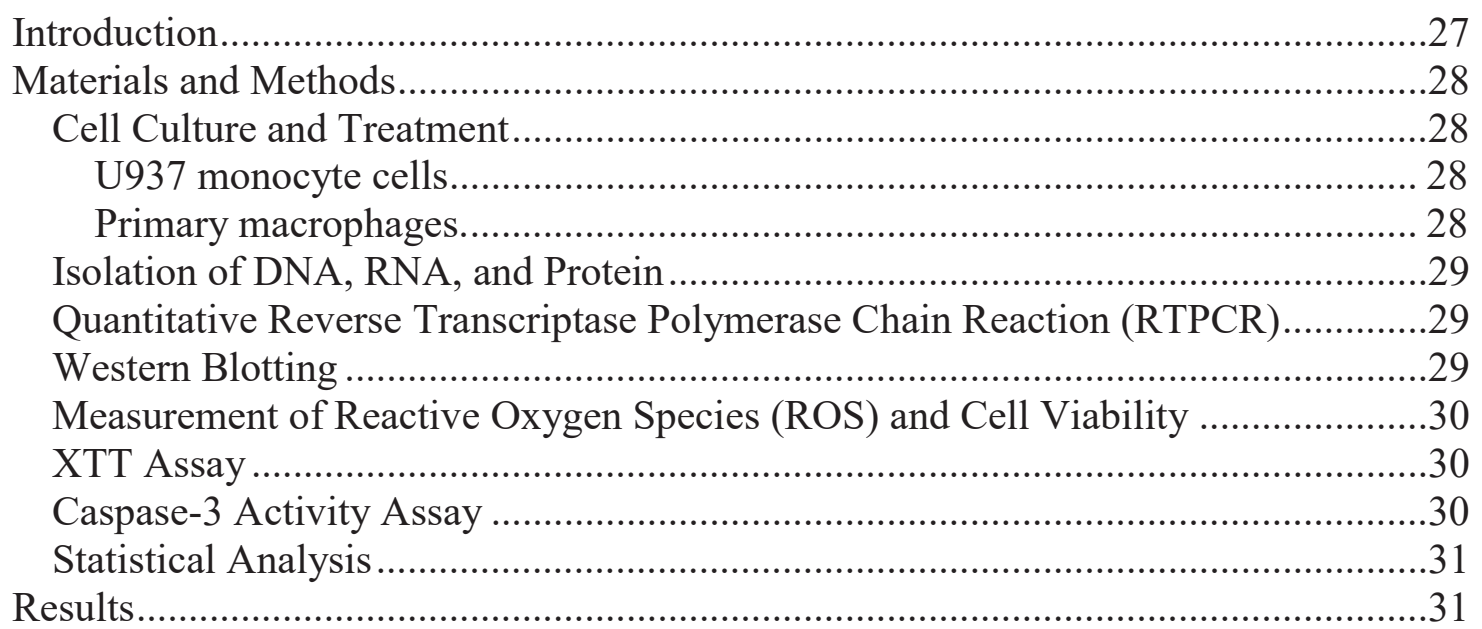

Effect of Acute Treatment of BaP, NPh, and Phe on CYP and AOE Expression, ROS and Cell Viability in U937 Cells ...................................................................... 31

Effect of Chronic Treatment of BaP, NPh, and Phe on ROS and Cell Viability .......31

Effect of Chronic Treatment of BaP, NPh, and Phe on Caspase-3 Activity ...............37

Effect of Chronic Treatment of BaP, NPh, and Phe on the Expression of CYPs at

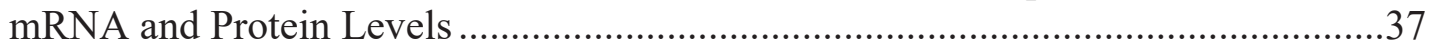

Effect of Chronic Treatment of BaP, NPh, and Phe on the Expression of AOEs at

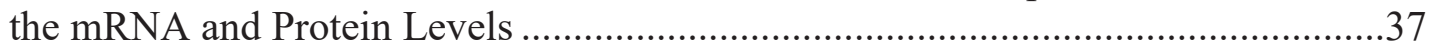

Effect of Chronic Treatment of BeA and BeF on ROS, Cell Viability, and

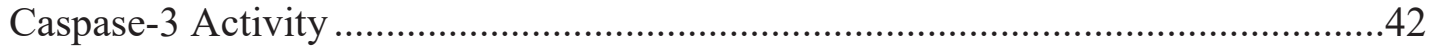

Effect of Chronic Treatment of BaP on CYP Expression, ROS and Cell Viability in Primary Macrophages .....................................................................................42

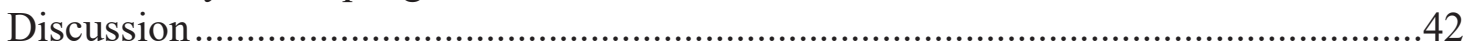

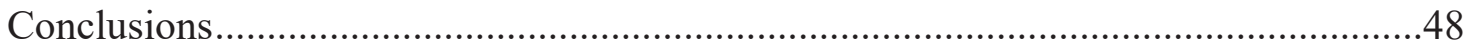

\section{CHAPTER 5. BENZO(A)PYRENE ENHANCES HIV-1 REPLICATION IN} MONOCYTIC CELLS .......................................................................................49

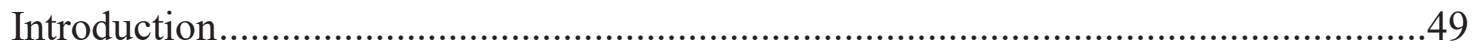

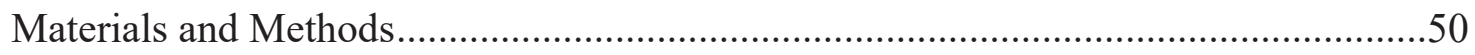

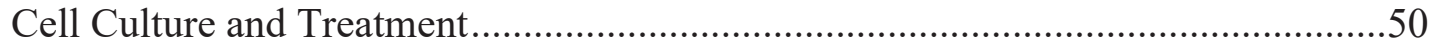

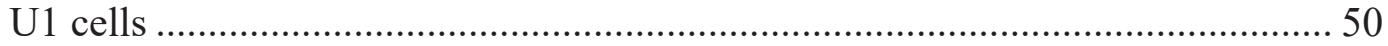

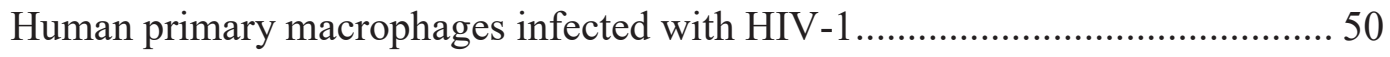

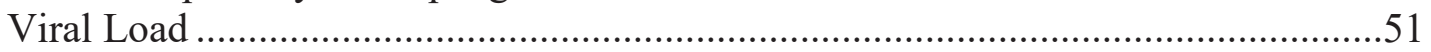

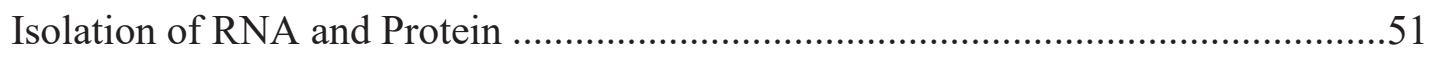

Quantitative Reverse Transcriptase Polymerase Chain Reaction (RTPCR)..............51

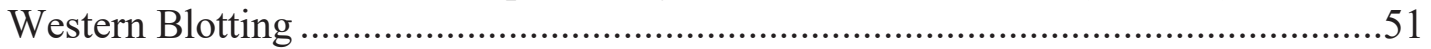

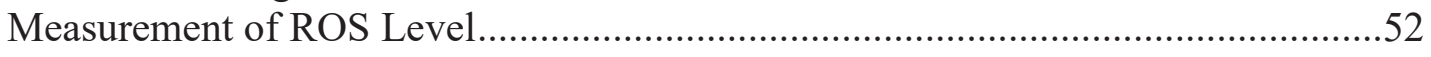

7-Ethoxy-Resorufin-O-Deethylase (EROD) Assay …..........................................52 


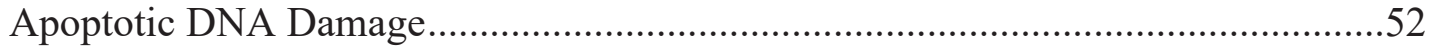

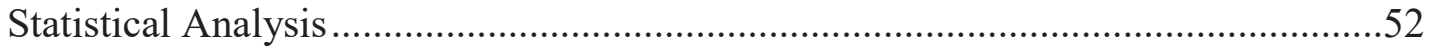

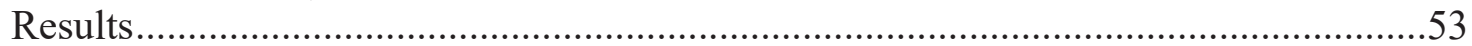

BaP Induces HIV-1 Replication in U1 Cells and HIV-1-infected Human Primary

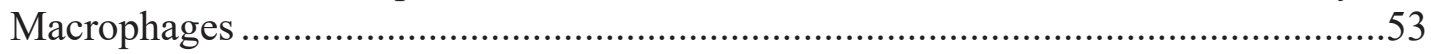

BaP Induces the Expression of CYP1A1 ..........................................................53

$\mathrm{BaP}$ Does Not Alter the Expression of AOEs ..........................................................53

Role of CYP1A1 on BaP-induced ROS Generation................................................57

Role of CYP1A1 and Oxidative Stress Pathways on BaP-induced HIV-1

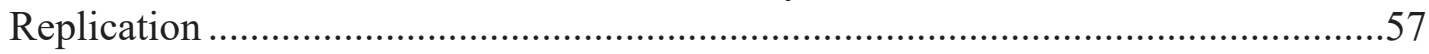

Nuclear Translocation of NF-кB Subunits upon BaP Exposure.................................57

Role of NF-кB Pathway in BaP-induced HIV-1 Replication .....................................57

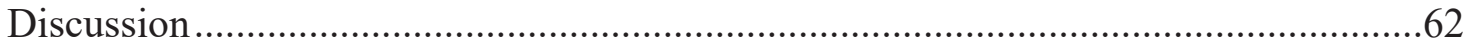

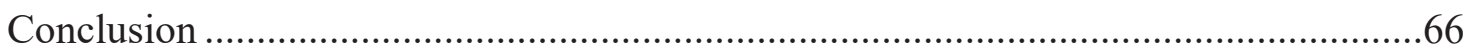

\section{CHAPTER 6. EXOSOMES FROM HUMAN PAPILLOMA VIRUS-INFECTED CERVICAL CANCER CELLS ENHANCE HIV-1 REPLICATION IN

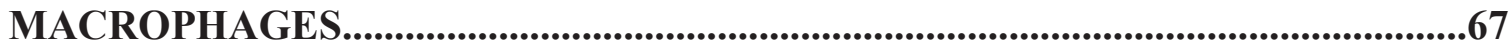

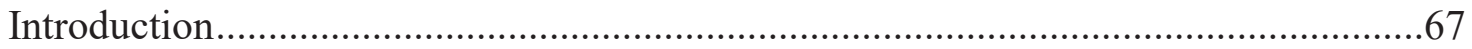

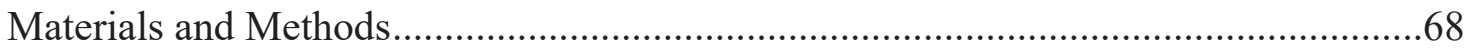

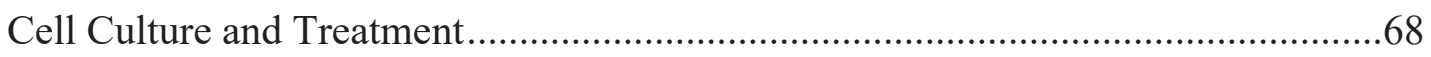

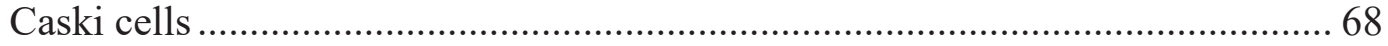

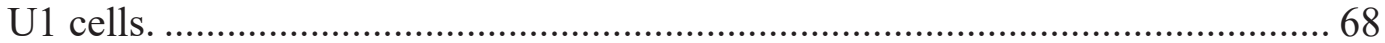

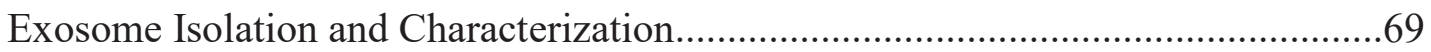

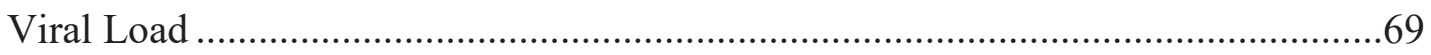

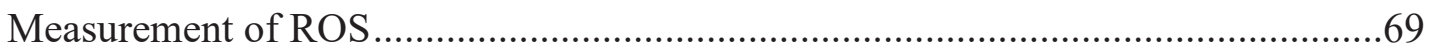

Total Antioxidant Capacity ...............................................................................

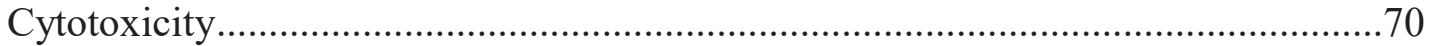

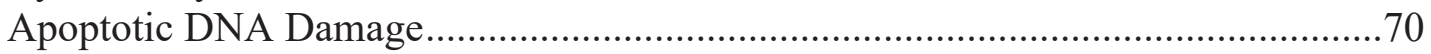

RNA and Protein Isolation.................................................................................

Quantitative Reverse Transcriptase Polymerase Chain Reaction (RTPCR)...............70

Western Blotting ............................................................................................

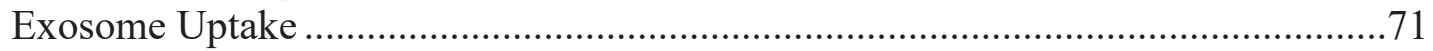

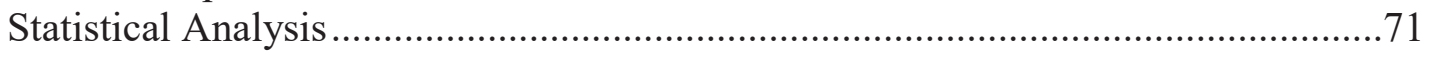

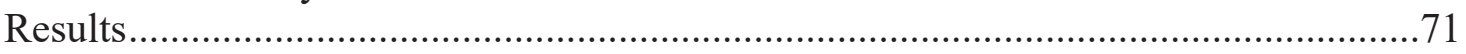

Cell Culture Supernatant from Caski Cells Enhances Oxidative Stress and Viral

Load in U1 Cells ..........................................................................................

Cell Culture Supernatant from Caski Cells Induces CYP Expression in U1 Cells ...74

Cell Culture Supernatant from Caski Cells Have No Significant Effect on AOEs

Expression in U1 Cells.........................................................................................

U1 Cells Uptake CCS-derived Exosomes Containing Oxidative Stress Factors........76

CCS-derived Exosomes Enhance Oxidative Stress and Viral Load in U1 Cells.......76

Treatment of Antioxidants, CYP-inhibitors, and Chemodietary Agents Reduce

Viral Load in Caski Exosomes-treated U1 Cells .......................................................82

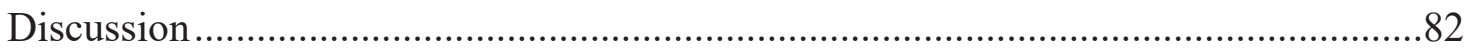

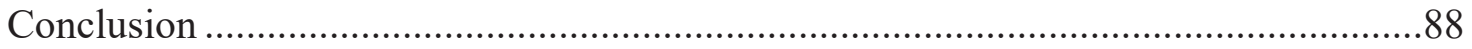


CHAPTER 7. CONCLUSIONS AND FUTURE DIRECTIONS.

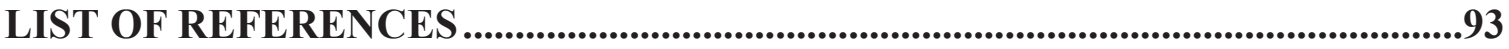

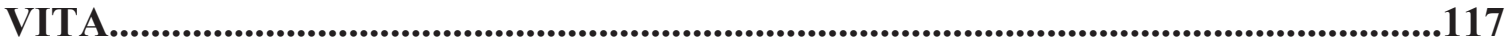




\section{LIST OF TABLES}

Table 2-1. Prevalence of smoking among HIV-1-infected population. .........................14

Table 2-2. Standard incidence ratio (SIR) for HPV-induced cervical cancer among women living with HIV-1 worldwide. ...................................................17

Table 2-3. HPV genotypes observed in HIV-1-postive women with cervical cancer. ..19 


\section{LIST OF FIGURES}

Figure 1-1. HIV-1 life cycle and antiretroviral drugs that act on its various stages.........5

Figure 3-1. Hypothesis: Polyaryl hydrocarbons in cigarette smoke and HPV-infected cervical cancer cells exacerbate HIV-1 replication via CYP-mediated oxidative stress pathway.

Figure 4-1. Effect of acute treatment of BaP, NPh, and Phe on ROS level (A) and cell viability (B) in U937 cells.

Figure 4-2. Effect of acute treatment of BaP (A), NPh (B), and Phe (C) on mRNA and protein expression of CYP1A1 in U937 cells.

Figure 4-3. Effect of acute treatment of BaP (A), NPh (B), and Phe (C) on mRNA and protein expression of CYP3A4 in U937 cells.

Figure 4-4. Effect of acute treatment of $\mathrm{BaP}(\mathrm{A}), \mathrm{NPh}(\mathrm{B})$, and Phe (C) on mRNA and protein expression of SOD1 in U937 cells.

Figure 4-5. Effect of acute treatment of BaP (A), NPh (B), and Phe (C) on mRNA and protein expression of catalase in U937 cells.

Figure 4-6. Effect of chronic (7 days) treatment of $\mathrm{BaP}, \mathrm{NPh}$, and Phe on reactive oxygen species (ROS) level (A-B) and caspase-3 activity (C) in U937 cells

Figure 4-7. Cell viability upon chronic treatment by $\mathrm{BaP}, \mathrm{NPh}$, and Phe measured by flow cytometry (A-B) and XTT assay (C).

Figure 4-8. Effect of chronic (7 days) treatment of BaP, NPh, and Phe on mRNA and protein expression of CYP1A1 (A) and CYP3A4 (B) in U937 cells...40

Figure 4-9. Effect of chronic (7 days) treatment of $\mathrm{BaP}, \mathrm{NPh}$, and Phe on mRNA and protein expression of AOEs SOD1 (A) and catalase (B) in U937 cells.

Figure 4-10. Cell viability (A), ROS level (B) and caspase-3 activity (C) upon chronic treatment by BeA and BeF in U937 cells.

Figure 4-11. Cell viability (A), ROS level (B) and mRNA expression level of CYP1A1 (C) and CYP3A4 (D) upon chronic treatment by BaP in primary macrophages.

Figure 5-1. Chronic treatment of BaP induces HIV-1 replication and apoptotic DNA damage in HIV-1-infected macrophages. 
Figure 5-2. Chronic treatment of BaP induces expression of CYPs in U1 cells.

Figure 5-3. Chronic treatments of BaP have no significant effect on the expression of AOEs in U1 cells.

Figure 5-4. Treatment of antioxidants and CYP1A1 inhibitors reduce ROS in U1 cells due to BaP exposure.

Figure 5-5. Treatment of antioxidants and CYP1A1 inhibitors reduce HIV-1 replication in $\mathrm{U} 1$ cells due to $\mathrm{BaP}$ exposure.

Figure 5-6. Translocation of NF-kB subunits from cytoplasm to nucleus upon BaP exposure.

Figure 5-7. Treatment of NFא-B inhibitors reduce HIV-1 replication in U1 cells due to $\mathrm{BaP}$ exposure.

Figure 5-8. Schematic diagram showing the mechanism of BaP-induced -1-1 replication in macrophages.

Figure 6-1. Caski cell culture supernatant (CCS) increases HIV-1 replication and oxidative stress in U1 macrophages.

Figure 6-2. Caski cell culture supernatant (CCS) enhances CYP expression in U1 macrophages.

Figure 6-3. Caski cell culture supernatant (CCS) alters antioxidant enzyme (AOE) expression in U1 macrophages.

Figure 6-4. U1 macrophages uptake CCS-derived exosomes (CCS-Exo) that contain oxidative stress factors.

Figure 6-5. CCS-derived exosomes (CCS-Exo) increases HIV-1 replication and oxidative stress in U1 macrophages.

Figure 6-6. Treatment of antioxidant, CYP inhibitors, and chemodietary agents reduce CCS or CCS-derived exosomes (CCS-Exo)-induced HIV-1 replication in U1 macrophages.

Figure 6-7. Proposed model for HPV-HIV-1 interaction via exosomes. .85 


\section{LIST OF ABBREVIATIONS}

\begin{tabular}{|c|c|}
\hline AHR & Aryl Hydrocarbon Receptor \\
\hline AIDS & Acquired Immunodeficiency Syndrome \\
\hline ART & Antiretroviral Therapy \\
\hline APOBEC & Apolipoprotein B mRNA Editing Enzyme, Catalytic Polypeptide-like \\
\hline Arnt & AHR Nuclear Translocator \\
\hline $\mathrm{AOE}$ & Antioxidant Enzymes \\
\hline ASK1 & Apoptosis Signal-regulating Kinase 1 \\
\hline $\mathrm{BaP}$ & Benzo(a)pyrene \\
\hline $\mathrm{BeA}$ & Benzo(a)antharacene \\
\hline $\mathrm{BeF}$ & Benzo(b)fluoranthene \\
\hline BHT & Butylatedhydroxy Toluene \\
\hline BHA & Butylatedhydroxy Anisole \\
\hline $\mathrm{CCS}$ & Cell Culture Supernatant from Caski Cells \\
\hline CCS-Exo & Exosomes Derived from Caski Cell Culture Supernatant \\
\hline CCR5 & C-C Chemokine Receptor Type 5 \\
\hline $\mathrm{CD} 4 / 8$ & Cluster of Differentiation $4 / 8$ \\
\hline $\mathrm{CDC}$ & Center for Disease Control and Prevention \\
\hline $\mathrm{C} / \mathrm{EBP} \beta$ & CCAAT Enhancer Binding Protein $\beta$ \\
\hline $\mathrm{CSC}$ & Cigarette Smoke Condensate \\
\hline CXCR4 & C-X-C Chemokine Receptor Type 4 \\
\hline CYP & Cytochrome P450 \\
\hline DC-SIGN & $\begin{array}{l}\text { Dendritic Cell-Specific Intercellular Adhesion Molecule-3-Grabbing Non- } \\
\text { Integrin }\end{array}$ \\
\hline Epi & Ellipticine \\
\hline ESCRT & Endosomal Sorting Complex Required for Transport \\
\hline gp120 & Glycoprotein 120 \\
\hline HIV-1 & Human Immunodeficiency Virus-1 \\
\hline $\mathrm{HPV}$ & Human Papilloma Virus \\
\hline HSIL & High-grade Squamous Epithelial Lesions \\
\hline IL $1-\beta$ & Interleukin 1-beta \\
\hline IKK-16 & IkB Kinase 16 \\
\hline IARC & International Agency for Research on Cancer \\
\hline LTR & Long Terminal Repeat \\
\hline MAPK & Mitogen-Activated Protein Kinases \\
\hline MHC I & Major Histocompatibility Complex \\
\hline MIP-1a & Macrophage Inflammatory Protein \\
\hline Nef & Negative Regulatory Factor \\
\hline NFAT & Nuclear Factor of Activated T cells \\
\hline $\mathrm{NF}-\kappa \mathrm{B}$ & Nuclear Factor Kappa-Light-Chain-Enhancer of Activated B Cells \\
\hline NMDA & N-Methyl-D-Aspartate \\
\hline $\mathrm{NPh}$ & Naphthalene \\
\hline Nrf-2 & Nuclear Factor Erythroid 2- related Factor \\
\hline NRTI & Nucleoside Reverse Transcriptase Inhibitor \\
\hline
\end{tabular}




$\begin{array}{ll}\text { NNRTI } & \text { Non- Nucleoside Reverse Transcriptase Inhibitor } \\ \text { PAH } & \text { Polyaryl Hydrocarbon } \\ \text { PBMC } & \text { Peripheral Blood Mononuclear Cells } \\ \text { PLWH } & \text { People Living with HIV-1 } \\ \text { PLWHA } & \text { People Living with HIV-1/AIDS } \\ \text { Phe } & \text { Phenanthrene } \\ \text { PI } & \text { Protease Inhibitors } \\ \text { PIC } & \text { Preintegration Complex } \\ \text { Pin } & \text { Pinnostilbene } \\ \text { P-TEFb } & \text { Positive Transcription Elongation Factor b } \\ \text { RANTES } & \text { Regulated on Activation, Normal T cell Expressed and Secreted } \\ \text { Res } & \text { Resveretrol } \\ \text { RT } & \text { Reverse Transcriptase } \\ \text { ROS } & \text { Reactive Oxygen Species } \\ \text { SOD1/2 } & \text { Superoxide Dismutase 1/2 } \\ \text { SiRNA } & \text { Small Interfering RNA } \\ \text { TAC } & \text { Total Antioxidant Capacity } \\ \text { TAR } & \text { Transactivation Response } \\ \text { Tat } & \text { Transactivator of Transcription } \\ \text { TB } & \text { Tuberculosis } \\ \text { TNF- } \beta & \text { Tumor Necrosis Factor-beta } \\ \text { UNAIDS } & \text { Joint United Nations Programme on HIV/AIDS } \\ \text { Vif } & \text { Viral Infectivity Factor } \\ \text { Vpr } & \text { Viral Protein R } \\ \text { Vpu } & \text { Viral Protein U } \\ \text { Vit C } & \text { Vitamin C } \\ \text { Vit E } & \text { Vitamin E } \\ \text { XRE } & \text { Xenobiotic Responsive Element } \\ \end{array}$




\section{CHAPTER 1. INTRODUCTION*}

\section{HIV-1 Prevalence and Epidemiology}

According to the surveillance report by Center for Disease Control and Prevention (CDC), 36.7 million people are currently living with HIV-1 worldwide and about the same number of people (35 million) have died since the start of the epidemic. The Joint United Nations Program on HIV-1/AIDS (UNAIDS) published a report in 2017, which revealed that the global prevalence of HIV-1 is $0.8 \%$ in adults. The report also revealed that, approximately $69.4 \%$ of the people living with HIV/AIDS (PLWHA) reside in the Sub-Saharan Africa, $13.8 \%$ in Asia and Pacific, 5.7\% in West and Central Europe and North America, 5.7\% in Latin America and Caribbean, 4.3\% in East Europe and Central Asia, and $0.6 \%$ in Middle East and North Africa [1]. In the United States alone, there were around 1.1 million PLWHA with 38,500 new infections and 6465 deaths due to HIV infection in the year 2015 (CDC report). However, the deaths resulting from HIV-1 infection dropped by 45\% between 2005 and 2015 (UNAIDS 2015 report). The decrease in HIV-1-related deaths may be attributed to the advancements in antiretroviral therapy (ART). It was reported that $62 \%$ of the PLWH received ART, $48 \%$ retained continuous treatment and 49\% achieved viral suppression in 2014 [2]. Although, there was a substantial decrease in deaths due to HIV-1, the rate of incidence of HIV-1 decreased only by $8 \%$ from year 2010 to 2015 . The incidence rate was observed highest among males $(I R=24.1)$, persons aged 25-34 $(I R=31.3)$ and black/African Americans $(I R=49 \%)$. The introduction of ART has indeed elongated the life span of PLWHA, however, they are now vulnerable to various HIV-1-associated comorbidities like cancer, tuberculosis and HIV-associated neurodegenerative diseases (HAND). The burden of cancer (especially non-Hodgkin's lymphoma, Kaposi's sarcoma, anal cancer and lung cancer) has substantially increased in PLWHA by about $50 \%$ in 2010 [3]. Cancer-related deaths in PLWHA have also increased by $8 \%$ within a decade from 2000 to 2010 [4]. Similarly, in a study conducted by Sactor et al. (2016), they observed that the frequency of HAND increased in PLWHA by 6\% from 2009 to 2012 [5].

\section{HIV-1 Biology}

\section{HIV-1 Structure}

HIV-1 is a primate lentivirus that belongs to a family of retrovirus. Retroviruses are capable to reverse-transcribe their RNA into DNA and incorporate the viral DNA into

\footnotetext{
* Portions of chapter 1 modified with permission. Ranjit, S., Kumar, S. Recent advances in cancer outcomes in HIV-positive smokers. F1000 Faculty Rev, 2018. 718

(doi:10.12688/f1000research.12068.1)
} 
the host genome, which is an important mechanism of sustenance for the viruses belonging to this category Lentivirus is a type of retroviruses with longer incubation period. HIV-1 is spherical in shape with a diameter of approximately $100 \mathrm{~nm}$. The virus comprises of two single-stranded RNA enclosed within a cone shaped capsid made of p24 proteins. The RNA comprises of three essential genes (gap, pol and env), two regulatory genes (tat and rev) and four accessory genes (vif, vpr, vpu, and nef) that encodes for altogether 15 proteins. Within the capsid, there are other proteins and enzymes such as reverse transcriptase, integrase, protease, vif vpr, nef, and p7, which are crucial for viral development. Surrounding the capsid is a matrix made of p17 protein, whose integrity is maintained by bilayered phospholipid membrane. The phospholipid membrane, which forms the viral envelope is embedded with 72 Env knobs, each comprised of trimer of gp41 topped with trimer of gp120. The gp41 trimer form a stem of Env protein, which is embedded into the lipid membrane, while gp120 forms a cap that protrudes out of the membrane [6].

\section{HIV Life Cycle}

Viral entry. HIV-1 entry to the host cells is initiated by binding of viral envelope protein, gp120 with CD4 receptors on the surface of the host cell membrane. Binding of gp120 with CD4 receptor changes the conformation of gp120, which facilitates its binding to host cell co-receptors such as CCR5 and CXCR4. This interaction exposes the gp41 subunit of the viral Env protein, which causes fusion of viral envelop and host cell membrane. The viral matrix and capsid are then phosphorylated by cellular kinases (e.g. MAPK kinases), which uncoats the capsid and releases its contents including the two single-stranded RNA, into the host cytoplasm [7]. HIV-1 enters the host cell through CCR5 coreceptors during the early stages of viral infection, while during the later stages of developing immunodeficiency, the virus tends to enter via CXCR4 coreceptors [8]. HIV-1 also enters macrophages through endocytic pathway in a CD4 and CCR5independent manner, especially via clathrin-mediated micropinocytosis. However, the viruses that enter the host cells via endocytosis have lesser infectivity, which is most likely due to the inactivation of viral particles within the endosomes and lysosomes [9]. Another mechanism of HIV-1 entry is via DC-SIGN (a C-type lectin expressed in the surface of dendritic cells), independent of CD4 receptor. Dendritic cells are present in peripheral mucosal tissues and have a major function as antigen- presenting cells in lymphoid organs. HIV-1 gp120 proteins binds to DC-SIGN lectins on the immatured dendritic cells. Upon maturation, the dendritic cells transport the DC-SIGN-bound viruses to the T-cells in the lymphatic organs [8].

Reverse transcription. Shortly after the virus fuses to the host plasma membrane, there occurs the formation of reverse transcription complex into the host cytoplasm, which comprises two single-stranded viral RNA, cellular lysine transfer RNA (tRNA Lys), reverse transcriptase (RT), integrase, capsid and cellular proteins and viral matrix. RT enzymes comprise of two components: DNA polymerase and RNase H. DNA polymerase uses cellular nucleotides to synthesize DNA strands. Cellular tRNA Lys binds to the primer binding site near the 5' terminal end of the viral RNA genome and 
serves as a primer for the synthesis of (-)-strand DNA by adding complementary nucleotides along the 5' end of the viral RNA genome. RNase H degrades sections of RNA from the RNA/DNA duplex, except for the purine-rich segments. These purinerich segments serve as primers for the synthesis of (+)-strand DNA using tRNA Lys bound (-)-strand DNA as a template [10]. After the formation of linear double stranded DNA, it forms a preintegration complex (PIC) with viral proteins (integrase, matrix, Vpr and capsid), which is translocated into the host nucleus via microtubule network [11].

Viral integration and transcription. Within the host nucleus, the preintegration complex is integrated into the host DNA by viral protein, integrase, preferentially near the DNAse-hypersensitive sites [12]. As the host cell is unable to distinguish between the proviral DNA and the DNA of its own, the host nuclear enzymes transcribe proviral DNA into viral messenger and genomic RNA. The LTR region of the proviral DNA contains binding sites for host cell nuclear transcription factors such as nuclear factor kappa B (NF- $\kappa \mathrm{B})$ and nuclear factor of activated T cells (NFAT) and RNA polymerase II. NF- $\kappa \mathrm{B}$ and NFAT are present in the cytoplasm in their inactive state, which translocate into the nucleus after activation by ROS, various cytokines or antigens. Binding of these transcription factors and RNA polymerase II at the proviral LTR region leads to the initiation of viral DNA transcription, forming short HIV-1 transcripts with RNA stem loop called transactivation response (TAR) element. The elongation of these short transcripts is facilitated by the binding of a viral transactivator protein, Tat and positive transcription elongation factor $\mathrm{b}(\mathrm{P}-\mathrm{TEFb})$ at the TAR element. The complex of Tat and $\mathrm{P}-\mathrm{TEFb}$ activates RNA polymerase II to elongate the viral transcripts. The viral mRNA and genomic RNA thus formed are exported from the host nucleus to the host cytoplasm.

Viral translation. Within the cytoplasm, the cellular enzymes translate the viral mRNA into viral proteins. Unspliced transcripts are used to synthesize Gag and Gag-ProPol proteins and spliced transcripts are used to synthesize Env, Nef, Tat, and Vpr. Larger polypeptides are cleaved by viral protein, protease into small functional proteins. Viral proteins such as Tat and Rev have functions in the transcription of proviral DNA within the nucleus, while viral Nef and Vpr alter the cellular environment for efficient viral replication. Nef downregulates the expression of CD4 and MHC I determinants on the surface of the infected cells, thereby protecting them from being engulfed by CD8 cytotoxic T cells. Nef also inhibits p53-mediated apoptosis of the infected cells [13] and promotes viral morphogenesis, budding, and infectivity [14]. During the budding stage, vif protects the virion from the antiviral activity of a cellular protein, APOBEC through polyubiquitination and proteosomal degradation [15].

Viral assembly and budding. All viral structural proteins can be derived from three polyproteins: Gag, Gag-Pro-Pol, and Env. Viral assembly occurs by clustering of 1500-2000 Gag proteins, 200 Gag-Pol-Pro, and 2 copies of viral RNA genome at the inner surface of the plasma membrane. Env proteins synthesized at the rough endoplasmic reticulum also translocate towards the viral budding site of the plasma membrane and package on the surface of the Gag after budding of the virion. The immature virion buds from the plasma membrane by hijacking the host cell endosomal sorting complex required for transport (ESCRT) [16]. After budding, the viral protease 
cleaves Gag and Gag-Pol-Pro proteins into functional proteins of a mature, infectious virion $[17,18]$. The schematic diagram for the HIV-1 life cycle and antiretroviral drugs that act on its various stages has been shown in Figure 1-1.

Viral latency. HIV-1 particles can reside within the cells in a dormant state without replicating, which is termed as viral latency. Viral latency may occur either via failure of PIC integration into the host gene or inhibition of the proviral DNA transcription. PIC integration may be interrupted if the nuclear transport of the complex is inhibited or if a weak PIC complex is synthesized due to poor reserve transcription [19]. Unintegrated PICs are often observed in viral reservoirs such as macrophages and they are capable of transcribing viral genes such as Nef and Tat [20]. If PIC is able to reach the nucleus, but binds to transcriptionally inactive heterochromatins, proviral DNA transcription does not occur [19]. Some cells lack transcription factors (NFKB, NFAT) and viral proteins (Tat), which impedes the proviral DNA transcription within them. While some cells, for example, monocytes contain high levels of anti-HIV-1 miRNA (miRNA-382, miRNA-150, miRNA-28, miRNA-223) that also inhibit the proviral DNA transcription [21].

\section{HIV Treatment Strategies}

HIV-1 mainly targets the immune cells with CD4 receptors such as T lymphocytes, monocytes, macrophages and dendritic cells [22]. After infection, the virus replicates within the cells leading to progressive loss of $\mathrm{CD} 4^{+} \mathrm{T}$ cells and immunosuppression. Over the time, the immune deficiency develops characteristic opportunistic infections and/or cancerous conditions called Acquired Immunodeficiency Syndrome (AIDS). With the introduction of antiretroviral therapy (ART), HIV-1 is no longer a life-threating disease but a chronic manageable disease. According to the recent

guidelines by the U.S. department of health and human services, ART treatment for HIV1 is recommended for all individuals with HIV-1, regardless of CD4 $4^{+}$cell counts [23].

ART does not cure or eradicate HIV-1, but suppresses the viral replication and minimizes the risk of viral transmission. There are 5 different classes of antiretroviral (ART) drugs approved by Food and Drug Administration (FDA) that are currently available commercially: 1. Nucleoside reverse transcriptase inhibitors (NRTIs) 2. Non-nucleoside reverse transcriptase inhibitors (NNRTIs) 3. Protease inhibitors (PIs) 4. Entry inhibitors, and 5. Integrase inhibitors [24].

NRTIs are the first FDA approved ARTs for HIV-1 treatment. As they have structural similarity to the cellular nucleosides, they compete with these natural nucleosides to make up viral DNA. Since, they do not have a 3'-hydroxyl group at the sugar moiety, the drugs targeting this hinder the formation of 3, 5'-phosphodiester bond between a NRTI and incoming 5'-nucleosides, thus interrupting the formation of viral DNA chain. On the other hand, NNRTIs binds to the noncatalytic allosteric site of the reverse transcriptase enzyme, which brings about a conformational change in its substrate binding site, leading to its reduced polymerase activity. NRTIs are administered as prodrugs, which require activation by cellular kinases to mediate its action, while 


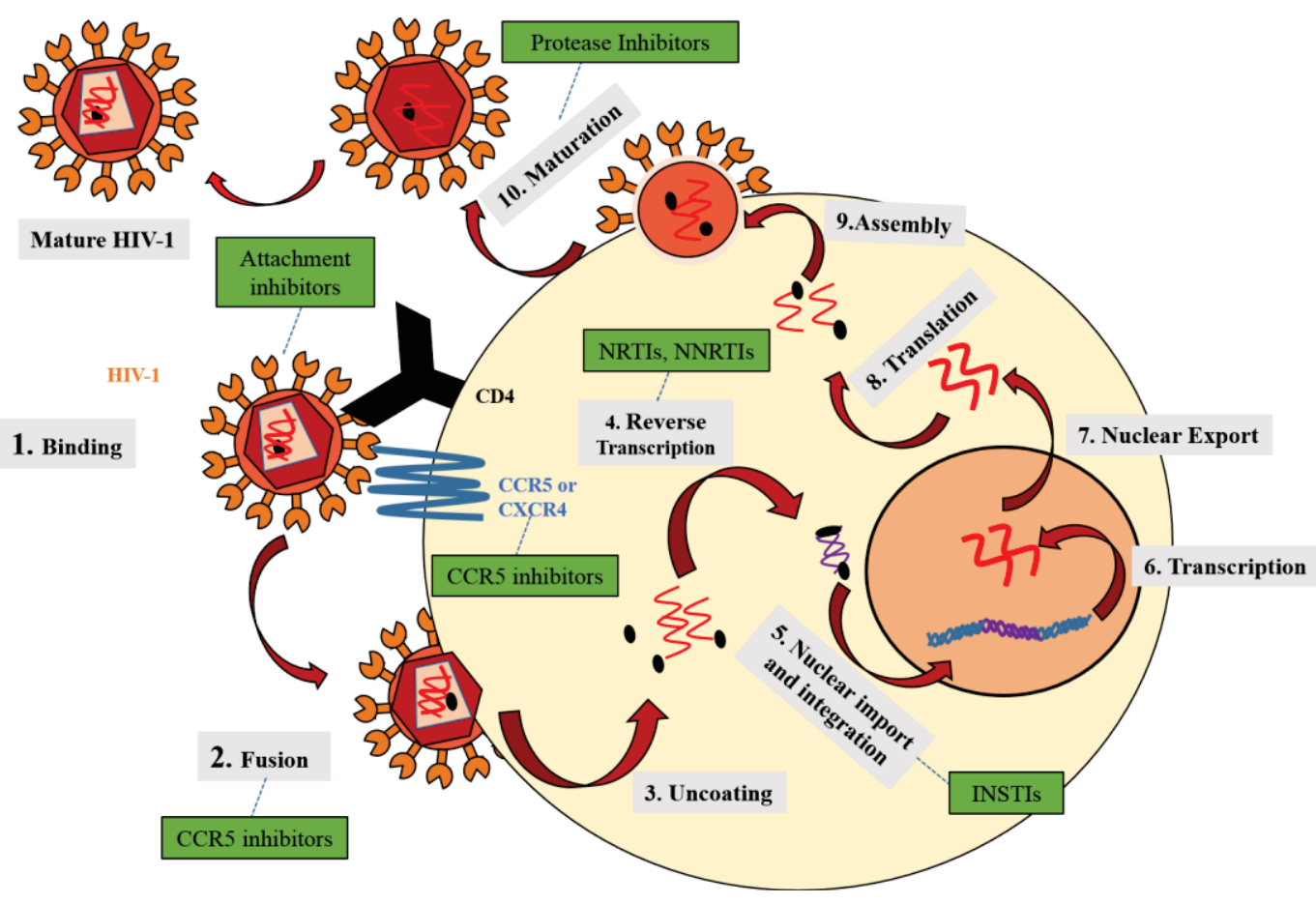

Figure 1-1. HIV-1 life cycle and antiretroviral drugs that act on its various stages. 
NNRTIs are administered in their active forms. NRTIs and NNRTIs include $50 \%$ of the commercially available ART drugs [25]. Integrase inhibitors acts by inhibiting the HIV-1 DNA strand transfer and integration into the cellular DNA. Protease inhibitors interfere with viral particle synthesis by blocking proteolysis of essential viral polyproteins, gag and gag-pol. Entry inhibitors are relatively new class of ART drugs that are subclassified into Fusion inhibitors, CCR5 antagonists, and post attachment inhibitors. By binding to the viral envelop proteins, fusion inhibitors block the formation of six helical bundle structure, which provides the force necessary for fusion pore formation in the cell membrane. CCR5 antagonists block viral entry by binding to the allosteric sites of CCR5 coreceptors thereby, changing its structural conformation such that the HIV-1 envelop protein, gp41 cannot recognize the binding site [26]. Post attachment inhibitors act after gp120 binds to CD4 receptors. Binding of these inhibitors changes the conformation of gp120-bound CD4 receptors such that it impedes the interaction of the later with the CCR5 and CXCR4 coreceptors, which is an important step for viral entry into the host cells [27]. Pharmacokinetic enhancer drugs such as cobicistat and ritonavir are also used in combination with other ART drugs. Although ritonavir has some antiviral activity, cobicistat does not have antiviral activity but it boosts the activity of other antiviral drugs (such as PIs and elvitegravir) by inhibiting CYP3A4 enzymes, which metabolize majority of the antiretroviral drugs [28].

\section{Drug Abuse}

Drug abuse is a major problem among PLWHA with approximately three-fold higher prevalence compared to the general population [29]. A study done by the NIH during 2005-2009 revealed that about one-third of the HIV-1 population were current drug users or alcoholics and $24 \%$ of them received medical treatment for drug abuse [30]. A cross sectional analysis study from the data obtained from 16 studies conducted at 14 sites across United States revealed that $47.7 \%$ of PLWHA are involved in alcohol use and $38.1 \%$ involved in illicit drug use. The report also suggested that $22.1 \%$ of the participants used more than one drugs of abuse [31]. Another report published by Gamarel et al. (2016) suggests that out of 2216 PLWHA they surveyed, 32.9\% frequently use tobacco, $27.5 \%$ use marijuana, $21.3 \%$ use alcohol, and $22.5 \%$ use other illicit drugs [32]. These drugs directly affect the pathogenesis of HIV-1/AIDS by enhancing immunosuppression or by activating cellular signaling pathways that enhance the viral replication [33]. As an indirect effect, substance abuse alters the mood, behavior, and capacity of judgement and decision making in PLWHA. This behavioral change indulges them in activities such as sharing needles, unprotected sex, and sex with multiple partners, which highly increase the risk of exposure to blood and other fluids of infected individuals and therefore HIV-1 transmission. The report by the NIH also showed that $16 \%$ of HIV - 1 transmission occurred through intravenous drug use and the remaining $84 \%$ via sexual contact, especially men having sex with men (MSM). Drug abuse also causes non-adherence to ART and drug-drug interactions resulting in reduced efficacy and increased toxicity of ART $[34,35]$. Moreover, PLWHA are usually involved in the use of more than one drugs of abuse [35], which directly act on central nervous system, causing neuroinflammation and neurotoxicity. The neurotoxic effects from multiple drug 
use further accentuates the neuronal damage caused by HIV-1 and increase risk of HIV1 -associated neurodegenerative diseases.

\title{
Smoking
}

The frequency of tobacco smoking is more than twice as high among PLWHA as it is in the general population [36]. Due to this increased prevalence, the deleterious effects of smoking on treatment outcomes for PLWHA have been investigated in several studies $[37,38]$. While smoking in the general population is known to enhance the risk of cancers and various respiratory and cardiovascular diseases, PLWHA face an even greater likelihood of developing life-threatening conditions related to tobacco use [39, 40]. Additionally, an analysis of patient populations provided with sufficient access to ART and quality healthcare has demonstrated that HIV-1-infected smokers display the highest rate of all-cause mortality compared to other HIV-1-infected individuals and population controls [41]. The explanation about how tobacco plays role in HIV-1 replication and pathogenesis will be discussed in detail in later sections.

\begin{abstract}
Alcohol
The prevalence of alcohol abuse among PLWHA is 2-4 times higher than the general population [42]. Approximately 40-50\% PLWHA are reported to have used alcohol, among which $\sim 28.8 \%$ and $\sim 10 \%$ are involved in harmful and hazardous drinking habits respectively [43-46]. High prevalence of heavy alcohol drinking is observed more among PLWHA who use one or more kinds of illicit drugs but the opposite has been observed in case of patients on ART with low CD4 counts [44, 47]. PLWH involved in hazardous drinking are $35 \%$ less likely to show adherence to ART, and the viral suppression among these patients is also $25 \%$ lesser than their non-drinking counter parts [44]. Alcohol also enhances the metabolism of ART drugs (such as PIs and NNRTIs) by upregulating the expression of drug metabolizing enzyme CYP3A4. The enhanced metabolism prevents ART to attain optimal concentrations at the target tissues and therefore reduces their efficacy [29]. Alcohol induces oxidative stress by upregulating the expression of CYP2E1, NAPDH oxidase, chemokines, and cytokines [29, 48-50] in hepatic cells, monocytes/macrophages, astrocytes, and neurons. The alcohol-induced oxidative stress causes liver damage and aggravates other comorbid conditions (like Hepatitis B and C infections, hypertension, and diabetes) in PLWH. Within the brain, the oxidative stress compromises the integrity of the blood-brain-barrier, making the brain tissues more vulnerable to neurotoxic effects of HIV-1 and other toxic substances. The alcohol-induced oxidative stress adds to the effect of HIV-1-induced oxidative stress, which further aggravate viral replication and apoptosis in brain cells. By enhancing the expression of various pro-inflammatory cytokines and chemokines within the brain tissues, alcohol promotes neuroinflammation and further elevate ROS generation. Alcohol exposure is also reported to cause glutamate toxicity in neurons through overstimulation of NMDA receptors [51]. Moreover, alcohol exposure is shown to potentiate the neurotoxic effects of HIV-1 proteins such as Tat, Nef, Vpr and gp120 [52,
\end{abstract}


53]. Several clinical studies have shown that alcohol use has both additive and interactive effects on mediating neuropathological conditions in HIV-1 patients, including HAND $[54,55]$.

\section{Marijuana}

Marijuana is the most commonly used illicit drug among PLWHA. Recent studies conducted on a cohort of HIV-1-infected patients receiving ART report that approximately $20-30 \%$ of the infected population are addicted to marijuana [32, 56-59]. A study conducted by Fogarty et al. (2007) reported that $\sim 60 \%$ of 408 HIV-1 infected patients in their study used marijuana; $55.7 \%$ of which reported to use it for recreational purpose, while $44.3 \%$ used marijuana for both recreational and medicinal purpose [60]. The effect of marijuana on adherence to ART and viral suppression in PLWH under care, is a disputed subject due to the mixed findings obtained in different cohorts of PLWH depending upon its use as illicit, medicinal, or recreational purpose. Some studies have revealed that non-adherence to ART is frequently observed in PLWH who are addicted to marijuana $[61,62]$. In contrast, some studies suggest that use of marijuana can have a positive effect on ART adherence through management of HIV symptoms and side effects of ART like anxiety, depression, fatigue, nausea, diarrhea, and peripheral neuropathy $[58,59,63]$. Interestingly, a recent study has revealed no significant association between the high-intensity marijuana use and ART adherence, suggesting that its use can rather be clinically beneficial for HIV symptom management [64]. Similarly, there is also a discrepancy on whether marijuana use affect the viral suppression in PLWH, with different studies showing contrast results [57, 61, 65]. However, a significant decrease in neurocognitive performance, learning, memory, and poor mental quality of life have been observed in PLWH who use marijuana in a moderate to heavy range, compared to those who used lightly or none [65-68]. Moreover, higher impact on learning and memory impairment have been observed among PLWH with early onset of marijuana (regular use prior to age 18) compared to the non-marijuana users or those with late onset of marijuana (regular use at 18 or later) [69]. Use of marijuana also leads to behavioral changes like involving in unprotected sex, which increases the probability of HIV-1 transmission [70].

FDA has approved $\Delta 9$-tetrahydrocannabinol $(\Delta 9$-THC), a major psychoactive component in marijuana, for the treatment of anorexia in PLWH. Clinical studies have also shown that its administration have minimal or very less effect on the viral load, $\mathrm{CD}^{+}$and $\mathrm{CD} 8^{+}$cell counts and protease inhibitor levels over a short-term treatment [71]. Chronic exposure of $\triangle 9$-THC in SIV-infected rhesus macaques showed that $\triangle 9$-THC does not enhance viral load or morbidity but attenuates the SIV progression through reduction of viral levels and inflammation [72]. However, the effect of $\Delta 9$-THC in SIVinfected rhesus macaques is sex-dependent. In case of female rhesus macaques, $\triangle 9$-THC did not show any reduction in viral load or $\mathrm{CD} 4^{+} / \mathrm{CD} 8^{+}$ratio; instead, it increased the expression of CXCR 4 in $\mathrm{CD}^{+} / \mathrm{CD}^{+}$lymphocytes prior to SIV inoculation [73]. With these mixed results, it is not clear if the use of marijuana can actually attenuate HIV-1 
progression in human, which highlights the need of further clinical investigation in this regard.

\section{Cocaine}

After Marijuana, cocaine is the second most used illicit drugs among PLWH with $8-15 \%$ PLWH involved in cocaine abuse $[31,74,75]$. Unlike marijuana, cocaine use has a negative impact on ART adherence. The adherence to ART is observed to be $\sim 3$-fold lower in cocaine users compared to non-users [76]. In addition, decreased ART response, increased viral load and $\mathrm{CD}^{+} \mathrm{T}$ cell depletion, and increased progression of AIDS have also been observed in PLWH who used cocaine [77, 78]. Furthermore, patients under ART are more prone to show increased cocaine toxicity through cocaine-ART interactions. Since, ART drugs such as PIs and NNRTIs are inducers/inhibitors of CYP3A4, they alter the CY3A4-mediated metabolism of cocaine. Enhanced formation of norcocaine, a hepatotoxic metabolite of cocaine leads to severe liver injury [34].

Cocaine abuse directly impacts HIV-1 replication in various immune cells by enhancing the viral entry into the host cells through upregulation of CCR5 and CXCR4 coreceptors and downregulation of chemokines (RANTES, MIP-1a and MIP-1b) that suppress HIV-1 entry into the host cell membrane [79, 80]. Cocaine also induces viral replication by enhancing the integration of PIC into host cell DNA [81]. Studies have also shown that cocaine negatively modulates anti-HIV miRNAs (such as miRNA-125b), which suppress HIV-1 replication in monocyte-derived dendritic cells by inhibiting the translation of HIV-1 transcripts [82]. In addition to its role in viral replication, cocaine exposure has also shown to cause apoptosis of $\mathrm{CD}^{+}$cells by inducing oxidative stress and mitochondrial depolarization [83].

Moreover, cocaine also plays a major role in the progression of HAND in PLWH. Higher extent of neurocognitive impairment and neurodegeneration, and faster progression to NeuroAIDS have been observed in PLWH who use cocaine compared to the non-drug users [84]. By compromising the integrity of BBB, cocaine increases the viral invasion into the brain tissues. More importantly, cocaine exposure not only enhances viral replication in macrophages and microglia but also in astrocytes, which are normally resistance to viral replication [85]. Cocaine also forms platelet-monocyte complex in the blood, which can easily cross the BBB and cause neuroinflammation. In addition, cocaine activates viral proteins such as Tat and gp120, which causes neurotoxicity by inducing oxidative stress, loss of mitochondrial potential, and neuronal apoptosis $[86,87]$

\section{Coinfections in HIV-1}

As HIV-1 weakens the immune system in HIV-1-infected individuals, they are susceptible to infections by various pathogens, including bacteria, fungus, protozoa, and

virus. The coinfection with these pathogens is also common in HIV-1 patients who are on 
ART because ART does not completely restore the immune response to normal levels. The most common types of coinfections as reported by National Institute of health (NIH) are tuberculosis, hepatitis B and C, and human papilloma virus (HPV) coinfections [88].

\section{Tuberculosis (TB)}

According to epidemiological data reported by WHO, the prevalence of TB is about 16-27 times higher among PLWH compared to general population [89]. The report also indicated TB to be the most common coinfection in PLWH and one of the major causes of HIV-1-related deaths [90]. The risk of TB is observed to be high among PLWH with higher viral load, lower $\mathrm{CD}^{+}$cell counts, and prior history of $\mathrm{TB}$, however, the risk is observed to be lower in patients who are under ART [91]. CD4 ${ }^{+} \mathrm{T}$ cells protect against M. tuberculosis by releasing proinflammatory cytokines; macrophages engulf $\mathrm{M}$. tuberculosis by phagocytosis and autophagy; $\mathrm{CD} 8^{+} \mathrm{T}$ cells inactivate $\mathrm{TB}$ antigen by lysing the antigen-presenting cells [92]. The number of these cells, (especially CD4 ${ }^{+} \mathrm{T}$ cells which are major cells for protecting against M. tuberculosis) are reduced in PLWH, which increase the risk and pathogenesis of TB in this population compared to the general population. Further, HIV-1 proteins such as Nef inhibits apoptotic response of macrophages against M. tuberculosis [93, 94].

HIV-1 viral load in lung tissues has been observed to be increased by 4-fold compared to the plasma viral load in PLWH coinfected with TB [95]. Similarly, macrophage cell lines (U937 and THP-1) coinfected with HIV-1 and M. tuberculosis also exhibited $\sim 3$-fold increase in viral load compared to their counterparts without TB infection [96]. Innate and adaptive immune response to M. tuberculosis release different proinflammatory cytokines (IL1- $\beta$, IL- 6 and TNF- $\beta$ ). These cytokines stimulate the nuclear transcription factors that cause the activation of HIV-1 LTR, thereby enhancing the transcription of HIV-1 genome [97-99]. Activated lymphocytes are shown to increase HIV-1 replication in HIV-1/TB coinfected alveolar macrophages through downregulation of CCAAT enhancer binding protein $\beta(\mathrm{C} / \mathrm{EBP} \beta)$ and upregulation of $\mathrm{NF}-\kappa \mathrm{B}$ in infected macrophages. These are nuclear transcription factors that inhibit and promote activation of HIV-1 long terminal repeat (LTR), respectively [97]. Innate immune response to TB has also been shown to increase the gene and protein expression of another nuclear transcription factor, nuclear factor of activated T cells 5 (NFAT 5), which also act on HIV-1 LTR leading to enhanced HIV-1 gene transcription [98]. Furthermore, release of cytokines in response to TB causes accumulation of T cells, which are major targets for HIV-1 infection $[100,101]$

\section{Hepatitis B and C}

Coinfection with hepatitis B virus (HBV) and hepatitis C virus (HCV) are common in PLWH. The prevalence of hepatitis B and $\mathrm{C}$ are 10-times and 6-times, respectively, higher in PLWH compared to the general population [102]. However, CDC reported that the incidence of $\mathrm{HCV}$ is approximately 3-times higher than hepatitis $\mathrm{B}$ 
among PLWH in USA [103]. The higher prevalence of HCV and HBV coinfection in PLWH is perhaps due to the common mode of transmission for these viruses through sharing of infected needles and sexual contact. However, HCV is more prevalent among PLWH who use intravenous drugs (72-95\%) compared to those who are MSM (1-12\%) or heterosexuals (9-27\%) [102]. With the depletion of CD4 ${ }^{+} \mathrm{T}$ cells in HIV-1 infection, there is further loss of HCV specific $\mathrm{CD}^{+}$and $\mathrm{CD} 8^{+} \mathrm{T}$ cell responses in HIV-1/hepatitis coinfection, which provides a favorable environment for $\mathrm{HCV}$ replication and pathogenesis [104, 105]. Further, HIV-1 envelop proteins such as gp120 directly enhance HCV replication by binding to CCR5 and CXCR4 coreceptors in hepatocytes and subsequently by upregulating cytokines like TGF- $\beta 1$, which have a stimulatory effect on HCV infection [106].

The effect of HIV-1/hepatitis virus coinfection in the progression of HIV-1 disease is not clear due to the conflicting evidences presented by different studies. Several studies conducted before ART era reported that HIV-1/hepatitis coinfection does not impact the progression of HIV-1 disease [107-110]. A study done in 9802 European HIV-1 cohorts revealed that HIV-1/HBV coinfection did significantly increase the liverrelated mortalities, but it did not have any effect on the progression of HIV-1/AIDS [111]. Another study done in a cohort of $328 \mathrm{HIV}-1$ patients under antiretroviral therapy also indicated no association of HCV on the mortality observed in PLWH [108]. In contrast, many studies conducted in ART era reported increased morbidity and mortality in HIV-1/hepatitis coinfected patients compared to patients infected with HIV-1 alone $[112,113]$. For example, a study conducted in 3111 Swiss HIV-1 cohorts under potent ART showed that HCV coinfection increased the clinical progression to AIDS and death rate in HIV-1/HCV coinfected patients, possibly via impaired CD4-cell recovery [112]. HIV-1 envelop proteins and the use of ART are also associated with severe hepatotoxicity, which could have increased the risk of hepatitis infections and subsequent morbidity and mortality in the HIV-1/hepatitis coinfected patients [106, 114]. Further, several studies have suggested the role of $\mathrm{HBV}$ protein $\mathrm{X}(\mathrm{pX})$ as transcriptional transactivator that lead to $\mathrm{HIV}-1$ replication in $\mathrm{CD}^{+} \mathrm{T}$ cells. HBV pX does not directly bind to HIV-1 LTR, but it activates HIV-1 transcription by stimulating the signaling pathways that activate transcription factors involved in $\mathrm{HIV}-1$ transcription (NF- $\kappa \mathrm{B}$, NFAAT) or by interacting with the basal transcriptional machinery [115].

\section{Human Papilloma Virus (HPV)}

Infection with low-risk HPV strains (type 6 and 11) only causes benign warts and condylomas, whereas infection with high risk HPV strains (type 16 and 18) leads to malignant diseases. The incidence of HPV among PLWHA is 1.5-times higher compared to general population. Both HPV 16 and 18 genotypes, which cause cervical cancer, are highly prevalent among PLWHA, with the predominance of the later genotype [116]. The incidence rate high risk HPV infection of cervix is approximately 3-times higher in HIV1-positive woman compared to HIV-1-negative women. Similarly, the progression of HPV to high-grade squamous epithelial lesions (HSIL) was also 2.55 times higher among HIV-1-positive women compared to HIV-1-negative women [117]. Several 
studies showed that progression of invasive cervical cancer in these patients increased with the decrease in $\mathrm{CD}^{+}$cell counts, suggesting a strong association of immunosuppression in the progression of the cancer [118-120]. Furthermore, many studies suggested that HIV-1 infection promotes HPV acquisition either by disrupting the mucosal epithelium or weakening the mucosal immune system, which facilitates HPV entry [121]. In addition to increased HPV acquisition, increased reactivation of latent HPV has also been observed in HIV-1-positive women [122]. HIV-1 proteins like Tat has also been reported to enhance transcription of HPV genome, leading to increased HPV replication [123].

On the other hand, several studies have indicated higher risk of HIV-1 acquisition in HPV-infected patients compared to uninfected individuals [124-126]. A study conducted by Averbach et al. (2010) indicated that HPV-infected women were 2.4-times more susceptible to HIV-1 infection compared to uninfected women [126]. Furthermore, another study suggested the involvement of specific strains of HPV, especially oncogenic HPV Type 16 and 18 in increasing HIV-1 acquisition among PLWHA [127]. However, the actual mechanism by which HPV induces the progression of HIV-1 disease is still unclear. HPV 16 oncoprotein E7 downregulates E-cadherin, an adhesion molecule in the genital epithelial layer [128]. HPV could possibly increase HIV-1 acquisition by interfering with the expression of E-cadherin. Immunological response to HPV accumulates $\mathrm{CD}^{+} \mathrm{T}$ cells at the site of infection, which are primary targets for HIV-1 infection [129]. More than 10-fold increase in the level of the cytokines (IL-6, IL-8, TNF- $\alpha$, MIP-1 $\alpha$ ) has also been observed during HPV infection [130]. Several of these cytokines including IL-6, IL-8, and TNF- $\alpha$ are shown to increase the expression of HIV-1 in host immune cells [131-133]. Massive oxidative stress occurs during HPV infection. HPV 16 oncoproteins (E6 and E7) and HPV 18 E2 proteins are known to cause chronic oxidative stress by a NADPH oxidase pathway or by increasing the mitochondrial ROS, respectively [134]. This increase in ROS could possibly activate ROS-sensitive nuclear transcription factors such as NF- $\kappa \mathrm{B}$, leading to the enhanced expression of HIV-1. 


\title{
CHAPTER 2. INTERACTION OF HIV-1 WITH TOBACCO SMOKING AND HPV INFECTION*
}

\author{
Tobacco Smoking
}

\section{Tobacco Prevalence in HIV-1 Patients}

In a cross-sectional survey conducted on 419,945 patients with HIV-1 in the US in $2015,42.4 \%$ were current smokers, $20.3 \%$ were former smokers, and $37.3 \%$ were nonsmokers [36]. According to the study, the prevalence of smoking among patients with HIV-1 (42.4\%) was more than two times higher than the uninfected US adult population $(20.6 \%)$. A similar trend of smoking prevalence was observed in a study that surveyed HIV-1-infected patients in New York in 2016, which found the association of smoking with poor health outcomes among PLWHA [135]. A study conducted in Ontario, Canada, in 2008 reported that $39.3 \%$ of the patients with HIV-1 were smokers, which declined by $1.6 \%$ by 2014 . However, the rate of smoking remained higher among HIV-1 population than the general population [136]. About 33\% of patients with HIV-1 in a study conducted on South Africans were also found to be smokers [136]. Furthermore, the prevalence of smoking is much higher among male HIV-1 patients who have sex with men (MSM) than it is among uninfected men [137-140]. A meta-analysis conducted by Jin et al. on Chinese PLWHA reported the prevalence of smoking to be $41.1 \%$ among the study population [141]. Table 2-1. summarizes the prevalence of smoking among patients with HIV-1 in different countries (most of the patients are from the US) from 2014 to 2017 [142-144], showing that smoking is highly prevalent in HIV-1-infected populations.

\section{Effect of Tobacco on HIV-1 Pathogenesis}

Combustion of cigarette smoke constituents generates large quantity of reactive oxygen species (ROS) such as superoxide, hydrogen peroxide, hydroxyl radical, and peroxynitrite, which are circulated through the blood to various tissues [145]. Excessive

\footnotetext{
* Portions of chapter 2 modified with permission. Ranjit, S., Kumar, S. Recent advances in cancer outcomes in HIV-positive smokers. F1000 Faculty Rev, 2018. 718 (doi:10.12688/f1000research.12068.1)

* Portions of chapter 2 modified with permission. Ranjit, S., Patters, B.J., Gerth, K.A., Haque, S., Choudhary, S., Kumar, S. Potential neuroprotective role of astroglial exosomes against smoking-induced oxidative stress and HIV-1 replication in the central nervous system. Expert Opinion on Therapeutic Targets, 2018. 22 (8)

(doi.org/10.1080/14728222.2018.1501473)
} 
Table 2-1. Prevalence of smoking among HIV-1-infected population.

\begin{tabular}{lclcc}
\hline \multicolumn{1}{c}{ Authors } & $\begin{array}{c}\text { Date of } \\
\text { publication }\end{array}$ & Location & $\begin{array}{c}\text { HIV-1 } \\
\text { patients (n) }\end{array}$ & $\begin{array}{c}\text { HIV-1 } \\
\text { smokers (\%) }\end{array}$ \\
\hline Mdodo et al. [36] & 2015 & United States & 419,945 & 42.4 \\
Hile et al. [135] & 2016 & United States & 14,713 & 40 \\
Bekele et al. [136] & 2017 & Canada & 4473 & 39.3 \\
Akhtar-Khaleel et & 2016 & United States & 6577 & 36.9 \\
al. [138] & 2014 & United States & 358 & 75 \\
Pacek et al. [142] & 2017 & Vietnam & 636 & 36.3 \\
Pollack et al. [144] & 2016 & South Africa & 146 & 33 \\
Murrison et al. & & & & \\
[146] & 2016 & Austria and & 447 & 49.4 \\
Brath et al. [143] & & Germany & \\
\hline
\end{tabular}


ROS causes oxidative stress that leads to oxidation of vital cellular components such as DNA, RNA, proteins, and lipids causing cytotoxicity and cell death. Furthermore, ROS also triggers the expression of pro-inflammatory cytokines and chemokines by activating the redox-sensitive nuclear transcription factor, NF- $\kappa \mathrm{B}$ [147]. The surge of proinflammatory cytokines and chemokines causes the recruitment of leukocytes, primarily monocytes and neutrophils, to the inflamed vascular endothelial cells [148]. Free radicals, elastase, and collagenase released from leukocytes, especially neutrophils, cause endothelial injury contributing to the development of atherosclerotic plaques. Oxidative stress induced by smoking and the subsequent complex pro-inflammatory cascade and atherosclerosis is linked to the pathogenesis of various chronic cardiovascular and respiratory disorders in smokers. As HIV-1 also causes oxidative stress [149-151], the oxidative damage is more pronounced in HIV-1 smokers, which increases the severity of the smoking-induced chronic diseases in PLWH. Higher incidence and severe forms of respiratory infections have been observed in HIV-1 smokers compared to HIV-1 nonsmokers and uninfected smokers [152]. The meta-analysis study done by Marshall et al. (2009) suggested that there was high incidence of respiratory infections and non-AIDSrelated mortality in HIV-1 smokers compared to HIV-1 non-smokers [140]. HIV-1 smokers mainly suffered death due to smoking-induced chronic diseases like stroke, asthma, COPD, coronary artery disease, peripheral vascular diseases, or cancers of organs like oropharynx, larynx, esophagus, lungs, cervix, stomach, bladder or kidney [37].

Studies have shown that smoking enhances HIV-1 replication in immune cells $[153,154]$. Recent studies from Kumar's lab demonstrated higher nicotine metabolism, HIV-1 viral load, ROS level and oxidative damage in HIV-1-positive smokers relative to HIV-1-positive non-smokers and uninfected smokers [153, 155]. Similar increase in HIV-1 viral load and oxidative stress have been reported in vitro in cigarette smoke condensate (CSC)-treated HIV-1-infected monocytic cells (U1 cells) or HIV-1-infected human primary cells [154]. We also observed significant increase in CYP enzyme expression: CYP2A6 and CYP3A4 in HIV-1 patients and HIV-1 smokers respectively; CYP1A1 and CYP1B1 in HIV-1-infected monocytic cells. When we inhibited the activity of these CYPs using specific CYP inhibitors or by specific siRNA knockdown, we observed a reduction in oxidative stress and viral load in CSC-treated cells, indicating the role of CYP enzymes in smoking-mediated oxidative stress and HIV-1 replication. Similarly, other studies have also revealed that CYP enzymes metabolize polyaromatic hydrocarbons in cigarette smoke into toxic metabolites that enhances HIV-1 gene expression by forming DNA adducts [156]. Another study showed that, tobacco enhances HIV-1 infectivity by up-regulating the expression of genes involved in viral replication (e.g. CD59 and TAX1BP1) as well as by down-regulating the genes that modulate cellular defense (e.g. TXNIP and PHGPX) and antigen presentation (e.g. MHC) [157].

\section{Effect of Tobacco on HIV-1 Treatment Outcomes}

Several studies have indicated that cigarette smoke decreases the response to ART $[158,159]$. However, it is not clear how tobacco use in PLWH impact ART efficacy. A 
study done by Feldman et al. (2006) demonstrated that there is higher rate of mortality $(53 \%)$ and morbidity (AIDS-defining cancer $=36 \%)$ among PLWH on ART who smoked compared to those who did not smoke [158]. The study findings emphasize nonadherence to ART as a possible cause for decreased response to ART among HIV-1 smokers. Another report by Miguez-Burbano et al. (2003) also showed that the immune and virologic response to ART decreased by $40 \%$ in HIV-1 smokers compared to HIV-1 non-smokers [160]. This study suggested that tobacco use may reduce ART efficacy by interfering with the metabolism of ART drugs. Tobacco induces the expression of various CYP enzymes such as CYP 1A1, 1B1, 2A6, and 3A4 in different cell types including monocytes and macrophages $[153,154]$. CYP3A4, which is a major drug metabolizing enzyme, metabolizes different ART drugs especially, NNRTIs and PIs [161]. Induction of CYP3A4 by tobacco may result in the enhanced metabolism of ARTs and consequently its reduced efficacy [159]. On the other hand, inhibition of CYPs by tobacco can also lead to decreased metabolism of the drugs, resulting in drug toxicity. Nicotine, an addictive substance in tobacco, also alter the expression of drug efflux transporters such as P-gp, MRP, and BCRP in the intestinal cells [162]. Alteration in the expression of these efflux transporters can have a direct impact on the absorption of orally administered ART drugs. As ART drugs are taken in combination, the drug-drug interactions with concomitant tobacco use can be even more complex.

\section{HPV Coinfection}

\section{HPV Prevalence in HIV-1-infected Patients}

The prevalence of any kinds of HPV infection is approximately 20-25\% higher in PLWH compared to general population [163, 164]. A survey conducted among African women with or without HIV-1 reported that the prevalence of any kind of HPV infection was about $72.2 \%$ in HIV-1-positive women, which was about $25 \%$ higher than the prevalence in uninfected women (47\%) [164]. Similarly, another study conducted on Italian women by Tartaglia et al. (2017) revealed that $48 \%$ of HIV-1-positive women had HPV compared to $28 \%$ of HIV-1-negative women [163].

High risk HPV that causes malignant diseases are more common compared to low risk HPV among HIV-1-infected individuals [165, 166]. High risk HPV type 16 and 18 are responsible for most of the cervical carcinoma in general population [166]. Table 2-2. shows the standard incidence ratio (SIR) for HPV-induced cervical cancer among HIV-1positive women all over the world, mainly in USA. The data in the table suggests that the average SIR for cervical cancer in HIV-1-positive women compared to uninfected women is 4.7 , which has remained fairly constant for almost the last two decades. Several studies have mentioned that the higher incidence and severity of cervical cancer in HIV1-positive women is associated with the decrease in the rate of $\mathrm{CD}^{+}$cell counts [119].

Joshi et al. (2014) reported that, the most common HPV genotypes causing

cervical intraepithelial neoplasia (CIN) stages 2 and 3 among Indian HIV-1-positve 
Table 2-2. Standard incidence ratio (SIR) for HPV-induced cervical cancer among women living with HIV-1 world wide.

\begin{tabular}{lclc}
\hline Authors & Year & Location & $\begin{array}{c}\text { SIR } \\
\text { (per 100,000) }\end{array}$ \\
\hline Massad et al. [172] & 2017 & United States & 3.31 \\
Chen et al. [173] & 2015 & Taiwan & 4.01 \\
Abraham et al. [119] & 2013 & North America & 3 \\
Massad et al. [174] & 2010 & United States & 1.32 \\
Chaturvedi et al. [175] & 2009 & United States & 8.9 \\
Engels et al. [176] & 2006 & United States & 4.2 \\
Clifford et al. [177] & 2005 & Global & 8 \\
Frisch et al. [178] & 2000 & United States & 5.3 \\
\hline
\end{tabular}


women were type $16(58.5 \%)$, type 31 (22.6\%), and type 18 and 68a (11.3\%) [167]. Similarly, a study conducted in HIV-1-infected women with CIN (stages 2 and 3) living in Botswana, indicated the presence of either HPV 16 or 18 or both in $51 \%$ of the participants [168]. However, Clifford et al. (2016) indicated higher prevalence of HPV types $16(42.5 \%), 18(22.2 \%), 45$ (14.4\%), and 35 (7.1\%) in HIV-1-positive women with invasive cervical cancer [166]. Table 2-3. demonstrates data from 9 different studies showing HPV genotypes observed in HIV-1-positive women with different stages of cervical cancer, living at different geographic locations. According to the data tabulated in Table 2-3, infection with multiple types of HPV are involved in the progression of cervical cancer. Infection with HPV type 16 and 18 has been observed commonly in HIV-1-positive women with ICC. In case of the HIV-1 patients with CIN, infection with HPV type 16 and other high-risk HPV such as 31, 35, 45, 52, and 58 are also commonly observed.

\section{Effect of HPV on HIV-1 Pathogenesis}

Several studies have indicated higher risk of HIV-1 acquisition in HPV-infected patients compared to uninfected individuals [124-126]. HPV-infected women are 2.4times more susceptible to HIV-1 infection compared to uninfected women [126]. The actual mechanism involving HPV-mediated HIV-1 acquisition is still not clear. HPV 16 oncoprotein E7 downregulates E-cadherin, an adhesion molecule in the genital epithelial layer [128]. HPV could possibly increase HIV-1 acquisition by increasing the permeability of the genital linings through interference with E-cadherin. Persistent HPV infections lead to preinvasive lesions and invasive cervical cancer. Large number of $\mathrm{CD}^{+}$and $\mathrm{CD}^{+} \mathrm{T}$ cells have been observed in the stromal infiltrates from invasive cervical carcinoma as well as from preinvasive lesions [169]. Accumulation of $\mathrm{CD}^{+}$and $\mathrm{CD}^{+} \mathrm{T}$ cells, which are major targets of HIV-1 infection, could increase the risk of HIV1 acquisition. Some studies have also shown the association of non-persistent HPV infections with the increased risk of HIV-1 acquisition [127]. The immune response for HPV clearance could also aggravate HIV-1 acquisition.

High levels of HIV-1 RNA has also been detected in the plasma of HIV-1infected women with high risk HPV cervical infection [170]. In an in vitro study done by Gage et al. (2000), there was a significant increase in viral load in U1 monocytic cells after treatment with supernatant collected from the cultured cervical biopsies and cervical cancer cell lines [171]. The study also suggested that cytokines released in response to HPV infection, especially IL-6, may be responsible for increased HIV-1 replication. More than 10-fold increase in the level of the cytokines (IL-6, IL-8, TNF- $\alpha$, MIP-1 $\alpha$ ) has also been observed during HPV infection [130]. Several of these cytokines including IL6 , IL-8, and TNF- $\alpha$ are shown to increase the expression of HIV-1 in host immune cells [131-133].

Massive oxidative stress occurs during HPV infection. HPV 16 oncoproteins (E6 and E7) are known to cause chronic oxidative stress by a NADPH oxidase pathway [134]. The oxidative stress caused by these HPV oncoproteins is shown to cause genomic instability 
Table 2-3. HPV genotypes observed in HIV-1-postive women with cervical cancer.

\begin{tabular}{|c|c|c|c|c|}
\hline Authors & Year & Location & HPV types & $\begin{array}{l}\text { Stage of HPV } \\
\text { infection }\end{array}$ \\
\hline Badial et al. [165] & 2018 & Brazil & $\begin{array}{l}56(17 \%) \text { and } 16 \\
(15.3 \%)\end{array}$ & \\
\hline Ortiz et al. [179] & 2017 & $\begin{array}{l}\text { Puerto } \\
\text { Rico }\end{array}$ & $\begin{array}{l}52(17.6 \%), 58 \\
(11.0 \%), 59(11.0 \%) \text {, } \\
16(9.9 \%) \text { and } 18 \\
(9.9 \%)\end{array}$ & \\
\hline Menon et al. [180] & 2016 & Kenya & $16(37 \%)$ and $18(24 \%)$ & ICC \\
\hline Clifford et al. [181] & 2016 & Africa & $\begin{array}{l}16(42.5 \%), 18 \\
(22.2 \%), 45(14.4 \%) \\
\text { and } 35(7.1 \%)\end{array}$ & ICC \\
\hline Joshi et al. [167] & 2014 & India & $\begin{array}{l}16(58.5 \%), 31(22.6 \%) \\
\text { and } 18 \& 68 \mathrm{a}(11.3 \%)\end{array}$ & $\begin{array}{l}\text { CIN stage } 2 \\
\text { and } 3\end{array}$ \\
\hline McKenzie et al. [166] & 2014 & $\begin{array}{l}\text { United } \\
\text { States }\end{array}$ & $\begin{array}{l}16(45 \%), 35(40 \%), \\
45(40 \%)\end{array}$ & $\begin{array}{l}\text { Cervical } \\
\text { dysplasia }\end{array}$ \\
\hline Marchetti et al. [182] & 2013 & Milan & $\begin{array}{l}16(19 \%), 31 \& 52 \\
(11 \%) \text { and } 18(4 \%)\end{array}$ & \\
\hline $\begin{array}{l}\text { Ramogola-masire et } \\
\text { al. [168] }\end{array}$ & 2011 & Bostwana & 16 and/or $18(51 \%)$ & $\begin{array}{l}\text { CIN stage } 2 \\
\text { and } 3\end{array}$ \\
\hline $\begin{array}{l}\text { Sahasrabudhhe et al. } \\
\text { [183] }\end{array}$ & 2007 & Zambia & $\begin{array}{l}52(37.2 \%), 58 \\
(24.1 \%), 53(20.7 \%), \\
16(17.2 \%) \text { and } 18 \\
(13.1 \%)\end{array}$ & $\begin{array}{l}\text { high-grade } \\
\text { squamous } \\
\text { intraepithelial } \\
\text { lesions }\end{array}$ \\
\hline
\end{tabular}


and DNA damage in infected cells. The oxidative DNA damage caused by HPV may enhance integration of HIV-1 DNA into the host DNA. Similarly, HPV 16 and 18 protein E2, which controls the HPV transcription, also contribute to ROS generation in mitochondria [184]. HPV 16 and 18 proteins E6, E1, and E2 are known to decrease the level and activity of several antioxidant enzymes and molecules like superoxide dismutase 2 (SOD2), catalase, and glutathione (GSH) [185, 186]. Significant increase in thioredoxin reductase and GST has also been reported in HPV 16 -infected dysplastic and neoplastic tissue samples [187]. Both, increase or decrease in the level of antioxidants is an indicative of the ongoing oxidative stress in the cells.

Cytokines like IL-6, IL-8, and TNF- $\alpha$ have the ability to stimulate nuclear transcription factors that regulate the transcription of HIV-1 DNA. TNF- $\alpha$ increases HIV1 expression by facilitating the nuclear translocation of $\mathrm{NF}-\kappa \mathrm{B}$, a nuclear transcription factor that binds to HIV-1 LTR and activate HIV-1 transcription [188]. IL-6 independently increases expression of HIV-1 proteins, facilitates viral transcription by enhancing RT activity, and in synergy with TNF- $\alpha$ it also helps to enhance the viral transcription [189]. Similarly, ROS such as superoxide and hydrogen peroxide are also known to activate NF- $\kappa B$ and cause reactivation of latent HIV-1 [190, 191]. Schreck et al. (1991) demonstrated that treatment of micromolar concentrations of hydrogen peroxide in Jurkat $\mathrm{T}$ cells enhances the nuclear translocation of $\mathrm{NF}-\kappa \mathrm{B}$ as well as binding of NF- $\kappa$ B to the HIV-1 LTR, leading to activation of the latent HIV-1 [192]. Another study done by Piette et al. (1994) also suggested the role of ROS as secondary messengers in activation of latent HIV-1 in monocytes and lymphocytes through activation of signaling pathways regulating HIV-1 transcription [190].

\section{Effect of Antiretroviral Therapy on HPV}

Studies suggest that PLWHA on ART show more rapid HPV clearance and higher rate of regression of cervical dysplasia and invasive cervical cancer compared to their counterparts who are not on ART [193]. ART is beneficial in clearing most of the oncogenic HPV, however some studies have mentioned that ART is not effective enough to clear HPV 16 and 18, which are the major strains causing cervical cancer [194]. A study by Clifford et al. (2016) showed that HIV-1-positive women receiving ART for $>2$ years showed high regression of cervical intraepithelial neoplasia compared to those who never received ART [195]. Another study by Konopnicki et al. (2013) also reported that ART significantly controlled high risk HPV, and it lowered the risk of even HPV 16 and 18 [196]. These studies suggested a strong role of ART on HPV clearance and alleviation of cervical lesions through restoration of immune response. A meta-analysis done by Kelly et al. (2017) with 31 studies, also concluded that patients on ART, showing sustained HIV-1 suppression and stable high $\mathrm{CD}^{+}{ }^{+}$cell count, have reduced incidence and progression of cervical lesions and cancer [197]. However, another meta-analysis study by Cobucci et al. (2015) suggested the opposite, i.e. higher risk of cervical cancer with ART treatment [198]. Most of these studies were performed in early ART era, when ART was initiated at lower nadir $\mathrm{CD}^{+}$cell count. In these studies, ART could not have caused the complete immune restoration in the patients, which could be a possible reason 
for the persistent HPV infection and cervical cancer progression [197]. Unlike smoking, HPV coinfection in HIV-1-infected patients do not have an adverse effect on ART prevalence and efficacy. 


\section{CHAPTER 3. SPECIFIC AIMS OF THE STUDY}

\section{Scientific Premise}

Antiretroviral therapy has prolonged the life span of PLWHA. However, the quality of life of this population has not improved much, because of the presence of comorbidities. Since HIV-1 causes immunosuppression, PLWHA are highly prone to opportunistic infections by various pathogens [199]. Furthermore, high prevalence of smoking, alcohol, and substance drug abuse has also been reported in this population [32]. PLWHA with these comorbidities are observed to have higher progression of HIV-1 disease despite ART use, mainly because of their impact on ART adherence, HIV-1 acquisition, and multiplication. In this study, we will be focusing mainly on smoking and HPV coinfection as comorbid factors, because of their high prevalence and high impact on HIV-1 replication in PLWHA.

The prevalence of tobacco smoking is 2-times higher in PLWHA compared to the general population [36], and is known to exacerbate HIV-1 pathogenesis [153, 154]. In vivo and in vitro studies have demonstrated that tobacco smoking is associated with decreased immune responses [200], increased inflammation [201] and oxidative stress $[202,203]$, and increased occurrence of opportunistic infections [160, 204]. In particular, tobacco smoking is known to accelerate HIV-1 replication in monocytes and macrophages, which are viral sanctuary sites where low concentrations of ART drugs fail to eliminate the virus $[154,205]$. HIV-1-infected macrophages can also infiltrate the brain and spread the virus to perivascular macrophages, leading to the development of neuro-AIDS. Previous studies have demonstrated that cigarette smoke condensate induces HIV-1 replication in monocytic cells via a CYP-mediated oxidative stress pathway [154]. Similarly, other studies have also revealed that CYP enzymes metabolize polyaromatic hydrocarbons in cigarette smoke into toxic metabolites that enhance HIV-1 gene expression by forming DNA adducts [156].

On the other hand, higher risk and prevalence of HPV infections that lead to cervical cancer, has also been reported in HIV-1-positive women [163, 164]. Presence of HPV coinfection in HIV-1-positive women has also been shown to exacerbate HIV-1 pathogenesis [124-126]. HPV-infected women are 2.4-times more susceptible to HIV-1 infection compared to uninfected women [126]. However, it is not clear how smoking or HPV coinfection in PLWHA enhance HIV-1 replication and pathogenesis. In the present study we will investigate the molecular mechanism by which smoking or HPV-infection induce HIV-1 replication in monocytic cells, particularly focusing on CYP and oxidative stress pathway.

In this study, we have used three different types of cell lines: U937, U1 and Caski Cells. U937 cells are monocytic cells derived from the histolytic lymphoma of a 37-yearold male patient and U1 cells are U937 cells chronically infected with HIV-1. As U1 cells comprise of HIV-1 incorporated into their genome but the cells are not capable of producing a new virus, they represent an ideal model for cells latently infected with HIV- 
1. Further, monocytes/monocyte-derived macrophages are one of the major targets and reservoirs for HIV-1 infection [206]. Once infected, these cells can travel through bloodstream and spread the virus in other organ systems such as brain [207]. Therefore, it is imperative to study HIV-1 replication in monocytes and monocyte-derived macrophages. We used U1 monocyte-derived macrophages, instead of U1 monocytes for the experimental purpose because monocytes lack cellular machinery required for HIV-1 transcription [208, 209]. Monocytes express high levels of transcriptionally inactive NF$\kappa B$ p50 homodimers, which are replaced by transcriptionally active NF- $\mathrm{kB}$ p50/p65 or p50/RelB as they differentiate into macrophages after stimulation by PMA [208]. Monocytes also lack cellular cofactors for HIV-1 Tat-mediated transactivation of viral LTR promotor, which is critical for HIV-1 transcription. However, the cofactors essential for HIV-1 Tat transactivation are abundantly expressed in monocyte-derived macrophages [209]. To confirm the results from the U937 and U1 cells, we used infected/uninfected human primary monocytes and macrophages. We also used Caski cells which are cervical cancer cells with integrated HPV 16 and 18 genomes. Although, there are other HPV-infected cell lines (e.g. Siha, hela, C33A), we particularly employed Caski cells because these cells have higher p53 expression, lower antioxidant genes expression, and higher extent of oxidative stress compared to other cell lines [210].

\section{Hypothesis}

We propose that smoking and HPV infection independently increase HIV-1 replication in monocytic cells latently infected with HIV-1, via Cytochrome p450 (CYP)mediated oxidative stress pathways. Cigarette smoke constituents especially polyaryl hydrocarbons (PAH) enhance the expression of CYPs (CYP 1A1, 1A2, 1B1), which in turn metabolize PAHs into various water-soluble metabolites. During the metabolism of PAHs, CYPs generate large amount of reactive oxygen species (ROS). The ROS thus generated acts as secondary messengers for activating signaling pathways that regulate HIV-1 replication. On the other hand, HPV-infected cervical cancer cells also contribute to increased oxidative stress in monocytic cells latently infected with HIV-1, by encapsulating oxidative stress factors, especially CYPs into exosomes and transporting them into the HIV-1-infected monocytic cells. These exosomes release the CYPs into the infected monocytic cells, which aids the endogenous CYPs to generate more ROS. The excess of ROS leads to oxidative stress and HIV-1 replication via a similar mechanism as explained in case of smoking. Hypothesis of the project has been illustrated in Figure 3-1.

\section{Clinical Significance and Impact}

The proposed project will help to find the mechanism by which comorbidities such as, smoking and HPV coinfection enhance HIV-1 replication in monocytic cells latently infected with HIV-1. Through the elucidation of the mechanism, the project will increase scientific understanding and help improve clinical practice in HIV-1 patients with tobacco addiction or HPV coinfection, by providing knowledge of the contribution 


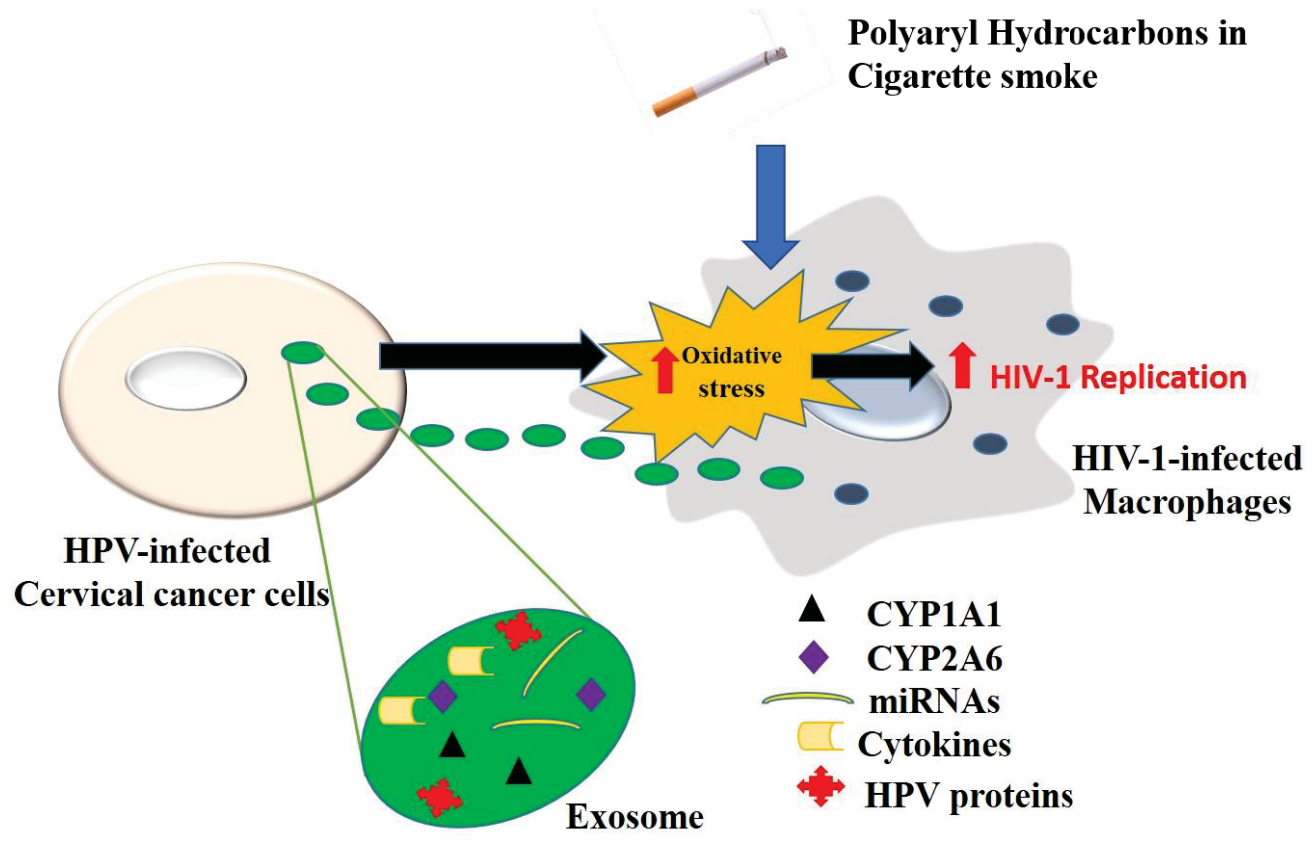

Figure 3-1. Hypothesis: Polyaryl hydrocarbons in cigarette smoke and HPVinfected cervical cancer cells exacerbate HIV-1 replication via CYP-mediated oxidative stress pathway. 
of monocytic and/or exosomal CYP enzymes in tobacco/HPV-mediated HIV-1 pathogenesis. The project would impact the treatment of HIV-1 in patients with these comorbidities, by providing a target for novel therapeutic interventions, adjustment of drug regimens, and potential application of exosomes as therapeutic carriers. This project would also provide new knowledge for exosomal biology thus impacting the understanding of other diseases and drugs of abuse.

\section{Objectives}

Our objectives are 1). To identify specific PAHs in cigarette smoke that cause oxidative stress and cytotoxicity in monocytic cells and show the potential role of CYPs in PAH-mediated cytotoxicity; 2). To demonstrate that PAHs cause HIV-1 replication in monocytic cells latently infected with HIV-1 via CYP-mediated oxidative stress pathway; 3 ). To show that exosomes from HPV-infected cervical cancer cells transfer oxidative stress factors, especially CYPs to the monocytic cells latently infected with HIV-1 and thereby induce HIV-1 replication via CYP-mediated oxidative stress pathway. Based on our objectives, we designed 3 aims for the study, which are described below:

\section{Aim 1: Study of Contribution of CYP Enzymes in PAH-enhanced Cytotoxicity in Monocytic Cells.}

We examined the acute (3-9 hours) and chronic (7 days) effects of five different carcinogenic PAHs such as Benzo(a)pyrene (BaP), Phenanthrene (Phe), Naphthalene $(\mathrm{NPh})$, Benzo(a)fluoranthene $(\mathrm{BeF})$, and Benzo(a)anthracene (BeA) on cytotoxicity in U937 monocytic cells. We also monitored ROS level, expression of CYPs (CYP1A1, CYP3A4) and antioxidant enzymes (AOEs: SOD1 and catalase) at mRNA and protein level and caspase-3 activity after PAH exposure and showed an association of the expression of CYPs and oxidative stress with PAH-induced cytotoxicity. Out of the five compounds tested, chronic exposure of BaP induced the highest level of cytotoxicity in U937 cells, which were associate with increased expression of CYP and oxidative stress, suggesting the potential role of CYPs in BaP-induced toxicity. We also confirmed these results in human primary macrophages.

\section{Aim 2: Study of Contribution of CYP Enzymes in BaP-enhanced HIV-1 Replication in Monocytic Cells Latently Infected with HIV-1.}

We examined if chronic exposure of BaP would increase HIV-1 viral load in U1 cells. As expected, we observed a significant increase in p24 levels in the supernatant of BaP-treated U1 cells, as measured by p24 ELISA. These results were also confirmed in $\mathrm{HIV}$-1-infected human primary macrophages. In order to see the involvement of CYPmediated oxidative stress in BaP-induced HIV-1 replication, we monitored ROS level and expression of CYPs (CYP1A1, CYP3A4) and AOEs (SOD1, Catalase) at mRNA and protein level. Furthermore, we established the role of CYPs and oxidative stress pathway 
in BaP-induced HIV-1 replication in U1 cells, by knocking-down the CYP1A1 gene using siRNA and treatment with selective CYP inhibitors and antioxidants. We also demonstrated that BaP-induced oxidative stress leads to the nuclear translocation of NF$\kappa B$ subunits (NF- $\kappa B$ p50 and p65), which was reduced by treatment with siRNA and antioxidants/CYP inhibitors. Treatment of specific NF- $\mathrm{kB}$ inhibitors (IKK-16 and SC540) significantly decreased HIV-1 replication in BaP-exposed U1 cells, indicating the specific role of NF- $\kappa \mathrm{B}$ pathway. Altogether, our results suggested that $\mathrm{BaP}$ enhances HIV-1 replication in U1 cells by activation of NF-KB through ROS generated by CYPmediated pathway.

\section{Aim 3: Study of Contribution of Exosomes Derived from HPV-infected Cervical Cancer Cells in Enhancing HIV-1 Replication in U1 cells.}

Initially, we examined whether supernatant from Caski cells can induce HIV-1 replication in U1 cells. We also monitored ROS level, total antioxidant capacity, cytotoxicity, DNA damage and expression of CYPs (CYP1A1, CYP2A6), and AOEs (SOD1, SOD2, catalase, PRDX6) after treatment of U1 cells with Caski cell culture supernatant (CCS). Our results suggested the association of CYPs and oxidative stress with CCS-induced HIV-1 replication. Next, we were interested to study if exosomes derived from CCS were responsible for the CCS-induced HIV-1 replication. We isolated exosomes from CCS using exosome isolation kit and characterized them by the methods described earlier [211]. We observed the presence of specific exosomal markers (CD63, CD81), CYPs (CYP1A1, CY2A6), AOEs (SOD1), and HPV-16 oncoprotein E6 in the CCS-derived exosomes (CCS-Exo). We also demonstrated that CCS-Exo diffused into $\mathrm{U} 1$ cells and induced oxidative stress and HIV-1 replication. By using specific CYP inhibitors (ellipticine and tryptamine) and antioxidant (resveratrol), we confirmed that both CCS and CCS-Exo induced HIV-1 replication in U1 cells via a CYP-mediated oxidative stress pathway. Furthermore, we also showed that treatment of chemo-dietary agents such as curcumin and cucurbitacin-D reduced HIV-1 replication in CCS and CCSExo-treated U1 cells. Overall, our results suggested the role of HPV infection on HIV-1 replication via exosome containing oxidative stress factors. 


\section{CHAPTER 4. EFFECT OF POLYARYL HYDROCARBONS ON CYTOTOXICITY IN MONOCYTIC CELLS*}

\section{Introduction}

According to the International Agency for Research on Cancer (IARC), there are around 5,300 chemicals identified in mainstream cigarette smoke, among which seventy are classified as carcinogens $[212,213]$. The IARC monograph program has listed several categories of chemical compounds that are potential carcinogens in cigarettes, including polyaryl hydrocarbons (PAHs), N-nitrosamines, aldehydes, phenols, volatile hydrocarbons, and other organic and inorganic compounds. Since the identification of cigarette constituents in 1950s, several studies have been conducted with regard to its carcinogenicity in different human organs. Tobacco-specific nitrosamines and PAHs are the most studied cigarette carcinogens. Tobacco-specific nitrosamines, 4(methylnitrosamino)-1-(3-pyridyl)-1-butanone (NNK) and N'-nitrosonornicotine (NNN) are reported to cause lung cancer and oral cavity cancer [214]. PAHs are associated with several cancers such as skin, lung, oral, and breast cancer [215-218]. Among the hundreds of PAHs present in the cigarette smoke, benzo(a)pyrene (BaP) is the most extensively studied PAH due to its known carcinogenic effects. Naphthalene (NPh) and phenanthrene (Phe) have relatively low carcinogenicity, but they contribute highly to the total PAH yield [219].

Cytochrome P450 (CYP) enzymes metabolize PAHs successively into epoxides and diol-epoxides [220]. These metabolites cause cellular damage either by forming DNA and protein adducts or by generating reactive oxygen species (ROS) [221, 222]. $\mathrm{BaP}$ is metabolized by CYP1A1 into a carcinogen, BaP-7,8-dihydrodiol-9,10-epoxide (BPDE), via a series of metabolic reactions. BPDE is reported to form DNA adducts which cause mutations in DNA, ultimately leading to carcinogenesis in lung and skin epithelial cells [223]. BaP is also reported to cause apoptosis in ovarian follicular cells via a CYP-mediated pathway [224]. Several reports in literature suggest the role of aryl hydrocarbon receptor-mediated CYP-induction and the subsequent oxidative stress in various forms of cancer and cardiac toxicity [225, 226]. Some studies reveal that BaP causes carcinogenic effects by inducing CYP1A1 expression through binding of p-53 to promoter region of CYP1A1 [227]. Rapid formation of [D10] r-1,t-2,3,c-4-tetrahydroxy1,2,3,4-tetrahydrophenanthrene ([D10] PheT), a carcinogenic diol metabolite of Phe, occurs in smokers [228]. Oxidative stress, DNA damage, and cell toxicity were observed following treatment with $\mathrm{NPh}$ in cultured J774A.1 macrophages [229].

* Reprinted with permission. Ranjit, S., Midde, NM., Sinha, N., Patters, BJ., Rahman, MA., Cory, TJ., Rao, PSS., Kumar, S. Effect of polyaryl hydrocarbons on cytotoxicity in monocytic cells: potential role of cytochromes $P 450$ and oxidative stress pathways. PLoS ONE, 2016. 11(9): e0163827.doi:10.1371/journal. pone.0163827 
Previous studies from our lab have shown that nicotine, a major constituent of tobacco, causes oxidative stress in U937-derived macrophages through a CYP2A6mediated nicotine metabolic pathway [230]. Our in vitro data was validated by an in vivo study in which HIV-1-positive smokers displayed a higher rate of nicotine metabolism by CYP2A6 than HIV-1-negative smokers [155]. HIV-1-positive smokers displayed a higher rate of nicotine metabolism by CYP2A6 than HIV-1-negative smokers [155]. The results from these studies were consistent with the findings from another study, in which there was an increase in viral load, cytokines, and oxidative stress, likely through CYP pathway, in the plasma and monocytes of HIV-1-infected smokers compared to HIV-1infected nonsmokers [153]. As the ultimate goal of the aforementioned studies was to explore the role of CYP enzymes in smoking-induced oxidative stress and HIV-1 pathogenesis, the experiments were conducted in monocytes/macrophages. Monocytes/ macrophages are one of the cellular targets for HIV-1 and they also serve as important viral reservoirs [231]. Smoking may enhance the infiltration of the infected monocytes/macrophages into the brain and further infect the microglia and astrocytes, ultimately leading to NeuroAIDS. However, it is still not clear whether other cigarette components besides nicotine are also responsible for CYP-mediated oxidative stress and HIV-1 replication in monocytes/macrophages. Therefore, the current study was designed to first examine the relative contribution of five $\mathrm{PAHs}$; $\mathrm{BaP}, \mathrm{NPh}, \mathrm{Phe}, \mathrm{BeA}$, and $\mathrm{BeF}$, on the regulation of CYP enzymes, oxidative stress, and cytotoxicity in U937 monocytic cells followed by primary macrophages.

\section{Materials and Methods}

\section{Cell Culture and Treatment}

U937 monocyte cells. The U937 monocytic cell line used for the study was obtained from ATCC (Manassas, VA). The cells were cultured in Roswell Park Memorial Institute (RPMI) 1640 media (Sigma Aldrich, St. Louis, MO), which included 1\% gentamycin (Mediatech Inc. Manassas, VA), L-glutamine (Fischer Scientific, PA), and sodium bicarbonate (Fischer Scientific, PA). To assess the acute effect of PAHs $(1 \mu \mathrm{M}$ $\mathrm{NPh}, 1 \mu \mathrm{M}$ Phe, and $100 \mathrm{nM} \mathrm{BaP}$ ) at 3, 6, and 9 hours, 0.8 million cells/well were seeded in a 12 -well plate. The cells were incubated overnight at $37^{\circ} \mathrm{C}$ in an incubator with $5 \%$ $\mathrm{CO}_{2}$ prior to treatment with PAHs. Following treatment, cells were collected the designated time points. To assess the chronic effect of PAHs (100 nM NPh, $100 \mathrm{nM}$ Phe, $100 \mathrm{nM} \mathrm{BeA}, 100 \mathrm{nM} \mathrm{BeF},(5,25$, and 100) nM BaP), 0.1 million cells were seeded per well in a 6-well plate. The cells were treated with PAHs after 30 minutes of incubation. The cells were treated every 12 hours for 7 days, with addition of $250 \mu$ of fresh media during each treatment to maintain the concentrations of PAHs. DMSO treated cells served as control for both the acute and chronic treatment paradigms.

Primary macrophages. Blood from interstate blood bank Inc. (Memphis, TN) was diluted with phosphate-buffered saline (PBS, Life Technologies, NY), layered on Ficoll (Mediatech Inc. Manassas, VA) and centrifuged at 400g for 30 minutes. The white 
ring of peripheral blood mononuclear cells (PBMCs) formed in between the plasma and Ficoll layers were carefully isolated. The PBMCs were washed with PBS several times to ensure the removal of Ficoll. The cells were incubated with ammonium-chloridepotassium (ACK) lysing buffer (Life Technologies, NY) at $4^{\circ} \mathrm{C}$ for 15 minutes to lyse and remove any red blood cells, if present. The clear pellets of PBMCs were then cultured in RPMI media with human serum and macrophage colony-stimulating factor for macrophage differentiation. The differentiated macrophages were treated with $\mathrm{BaP}$ (100 $\mathrm{nM}$ ) for 6 days, every 24 hours with an addition of $500 \mu \mathrm{l}$ fresh media after each treatment.

\section{Isolation of DNA, RNA, and Protein}

DNA, RNA, and protein were isolated from the lysed cells using Allprep DNA/RNA/Protein QIAGEN Kit (Valencia, CA) using the supplier's protocol. RNA and DNA were quantified using Nanodrop 2000c UV-Vis Spectrophotometer (Thermo Fischer Scientific, Rockford, IL) by measuring their absorbance at $260 \mathrm{~nm}$. The protein was quantified using the BCA protein assay kit (Thermo Fischer Scientific, Rockford, IL).

\section{Quantitative Reverse Transcriptase Polymerase Chain Reaction (RTPCR)}

Quantitative RTPCR was performed to measure the relative mRNA fold expression of the CYPs 1A1, 3A4 and the antioxidant enzymes (AOEs) superoxide dismutase 1 (SOD1) and catalase in U937 cells upon exposure to PAHs. Purified RNA (120 ng) was reverse transcribed to cDNA using a SimpliAmp Thermal Cycler (Applied Biosystems, Foster City, CA). The cDNA was amplified in a Step-One Plus Real-Time PCR System (Applied Biosystems, Foster City, CA) using TaqMan Gene Expression kit (Applied Biosystems, Foster City, CA). The following probes from Applied Bioscience were used for the Q-RTPCR reaction: CYP1A1 (Hs01054794_m1), CYP3A4 (Hs00430021_ml), SOD1 (Hs00533490_ml) and catalase (Hs00156308_ml). The 2- $\Delta \Delta \mathrm{Ct}$ method was used to calculate the relative mRNA fold expression of the genes, using glyceraldehyde 3-phosphate dehydrogenase (GAPDH) as an endogenous control.

\section{Western Blotting}

To determine the expression of proteins of interest, $30 \mu \mathrm{g}$ of proteins in 5\% SDS were separated on a polyacrylamide gel (4\% stacking, $10 \%$ resolving gel) at $150 \mathrm{~V}$ for 70 minutes. The proteins from the gel were transferred to a polyvinylidene fluoride membrane at $0.35 \mathrm{Amp}$ for 90 minutes. The transferred blots were blocked with $5-10 \mathrm{ml}$ of Li-Cor blocking buffer (LI-COR Biosciences, Lincon, NE) for 1 hour and incubated overnight with primary antibodies (GAPDH Rabbit Mab, 1:2000 dilution, Cell Signaling Technology, Danvers, MA; CYP1A1 rabbit Mab, 1:200 dilution, Abcam, Cambridge, MA; CYP3A4 Mouse Mab. 1:200 dilution, Santa Cruz Biotechnology. Inc. Dallas, TX; 
SOD1 Mouse Mab, 1:1500 dilution, Santa Cruz Biotechnology. Inc. Dallas, TX; Catalase Mouse Mab, 1:1200 dilution, Santa Cruz Biotechnology. Inc. Dallas, TX) at $4^{\circ} \mathrm{C}$. After subsequent washing, the blots were incubated with corresponding secondary antibodies (1:10000 dilution, Goat anti-Mouse Mab, LI-COR Biosciences, Lincon, NE; 1:10000 dilution, Goat anti-Rabbit Mab, LI-COR Biosciences, Lincon, NE) for 1 hour at room temperature. The blots were scanned with Li-Cor Scanner (LI-COR Biosciences, Lincon, $\mathrm{NE}$ ) and the densitometry data obtained from Image Studio Lite version 4.0 were used to calculate the fold expression of the proteins. GAPDH was used as an internal loading control to normalize the expression of sample proteins.

\section{Measurement of Reactive Oxygen Species (ROS) and Cell Viability}

ROS and cell viability were quantified by flow cytometry using the fluorescence dye 5-(and-6)-chloromethyl 2',7'- dichlorodihydrofluorescein diacetate (CM-H2DCFDA) (Life Technologies, Oregon, USA) and Ghost Dye (Tonbo Biosciences, San Diego, CA), respectively. The treated cells were thoroughly washed with PBS and resuspended in 1 $\mathrm{mL}$ of PBS containing 2\% FBS supplemented with 2-5 $\mu 1$ of CM-H2DCFDA and $1 \mu \mathrm{l}$ of the Ghost dye. The cells were then incubated at room temperature in the dark for 30 minutes and subsequently washed and resuspended in $300 \mu 1$ of PBS containing $2 \%$ FBS. Dichlorodihydrofluorescein (DCF) emission at $525 \pm 20 \mathrm{~nm}$, which is proportional to the ROS generated in the cells, and emission of Ghost dye at $780 \mathrm{~nm}$, which is proportional to the cell viability, were detected by flow cytometry (BD Biosciences, San Jose, CA). The data were analyzed by using the BD FACS software version 8 .

\section{XTT Assay}

Cell viability was also measured using the XTT assay kit (Cell Signaling Technology Inc., Danvers, MA). The PAH-treated cells (0.12 million) were suspended in $200 \mu \mathrm{l}$ of phenol red-free media. XTT detection solution was made by mixing electron coupling solution and XTT reagent in the ratio of 1:50. Fifty microliters of the XTT detection solution was added to each well of the 96 -well plate containing $200 \mu 1$ of the cell suspension and the plate was incubated at $37^{\circ} \mathrm{C}$ for three hours. The absorbance measured at $450 \mathrm{~nm}$ represented cell viability.

\section{Caspase-3 Activity Assay}

Caspase-3 activity was measured using Caspase-3 colorimetric assay kit (BioVision, Inc., Milpitas, CA). Cells (1-5 millions) obtained after the treatments were lysed and protein was extracted. About $100 \mu \mathrm{g}$ of the protein was diluted to a final volume of $50 \mu \mathrm{l}$ in cell lysis buffer. Each protein sample was added to $50 \mu 1$ of $2 \mathrm{X}$ reaction buffer (containing $10 \mathrm{mM} \mathrm{DTT}$ ) and $5 \mu 1$ of $4 \mathrm{mM}$ DEVD-pNA substrate and the reaction mixture was incubated at $37^{\circ} \mathrm{C}$ for 1 hour. Absorbance of the samples was measured at $405 \mathrm{~nm}$. 


\section{Statistical Analysis}

For the statistical analysis of the data obtained from RTPCR, Western blot, cell viability assay, ROS level, and caspase- 3 activity assay, mean \pm SEM was calculated and One-way ANOVA was applied to calculate $p$ values. A $p$ value of $\leq 0.05$ was considered significant.

\section{Results}

\section{Effect of Acute Treatment of BaP, NPh, and Phe on CYP and AOE Expression, ROS and Cell Viability in U937 Cells}

Initially, we monitored the effect of the acute exposure of $\mathrm{BaP}, \mathrm{NPh}$, and $\mathrm{Phe}$ in U937 cells. The cells were treated with BaP $(100 \mathrm{nM}), \mathrm{NPh}(1 \mu \mathrm{M})$, and Phe $(1 \mu \mathrm{M})$ for 3,6 , and 9 hours. There was no significant change in ROS and cell viability in cells treated with the PAHs for 3 and 6 hours (Figure 4-1A-B). However, a significant decrease $(\mathrm{p} \leq 0.05)$ of $\sim 10 \%$ in ROS was observed with all the three compounds after 9 hours of exposure (Figure 4-1A). The cell death at 9 hours was not particularly different from the control. The small decrease in ROS would not have been sufficient enough to cause a significant impact on cell viability. In general, there was minimal to no effect of acute exposure of $\mathrm{BaP}, \mathrm{NPh}$, and Phe on oxidative stress and cytotoxicity in U937 cells.

Furthermore, there was no significant effect of the acute exposure of the PAHs over the induction of CYPs (Figures 4-2 and 4-3) and AOEs (Figures 4-4 and 4-5). Only $\mathrm{BaP}$ exposure showed a significant ascending trend in the mRNA expression of CYP1A1 over time $(\sim 10$ fold increase at 6 hours to $\sim 30$ fold increase at 24 hours, $p \leq 0.05)$, as well as with increasing concentration (Figure 4-2A). However, there was no significant increase in the expression of CYP1A1 protein with either $100 \mathrm{nM}$ or $1 \mu \mathrm{M} \mathrm{BaP}$ treatment. None of the PAHs showed significant change in either the mRNA and protein expression levels of CYP3A4, except for the treatment with Phe $(1 \mu \mathrm{M})$, which showed $\sim 3$ fold increase in CYP3A4 expression at mRNA level at 12 hours $(\mathrm{p} \leq 0.05)$ (Figure 4-3C). At 24 hours, we observed a significant increase in the SOD1 mRNA expression with $(1 \mu \mathrm{M}) \mathrm{BaP}(\sim 3$ fold, $\mathrm{p} \leq 0.05)$ (Figure 4-4A). None of these compounds showed significant alteration in the protein expression levels of SOD1. Similarly, no significant change in the mRNA and protein expression levels of catalase was observed with any of the acute PAH treatments (Figure 4-5).

\section{Effect of Chronic Treatment of BaP, NPh, and Phe on ROS and Cell Viability}

The cells were treated with three different concentrations of $\mathrm{BaP}(5 \mathrm{nM}, 25 \mathrm{nM}$, and $100 \mathrm{nM}$ ), $100 \mathrm{nM} \mathrm{NPh}$, and $100 \mathrm{nM}$ Phe for seven days to examine the chronic effect of the PAHs on ROS generation and cell viability. A significant concentration-dependent 


\section{A. ROS Level}

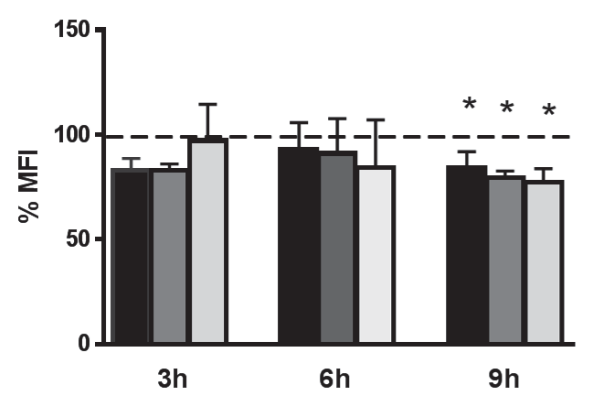

B. Cell Viability

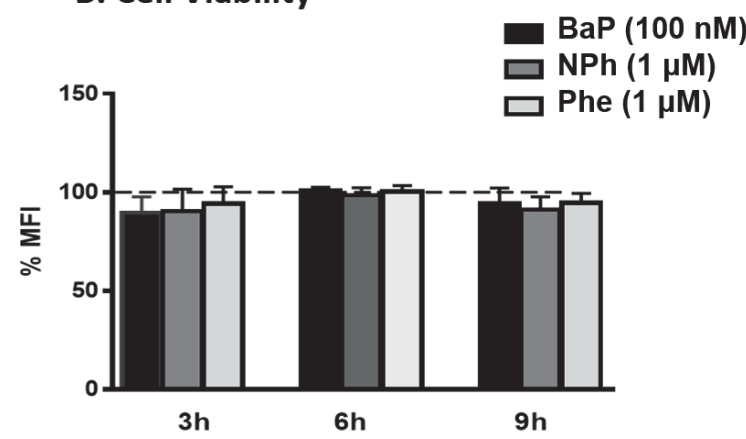

Figure 4-1. Effect of acute treatment of BaP, NPh, and Phe on ROS level (A) and cell viability (B) in $\mathrm{U} 937$ cells.

The U937 cells were treated with $100 \mathrm{nM} \mathrm{BaP}, 1 \mu \mathrm{M} \mathrm{NPh}$, and $1 \mu \mathrm{M}$ Phe for 3, 6, and 9 hours. ROS level and cell viability were measured using flow cytometry. Measured values at every time point were normalized to control that was set at $100 \%$. X-axis and Y-axis correspond to time points and \% of mean fluorescent intensity (\%MFI), respectively. The data are presented as a mean \pm SEM of three independent experiments. $*$ represents $\mathrm{p} \leq 0.05$ compared with the control group. 


\section{CYP1A1}

A

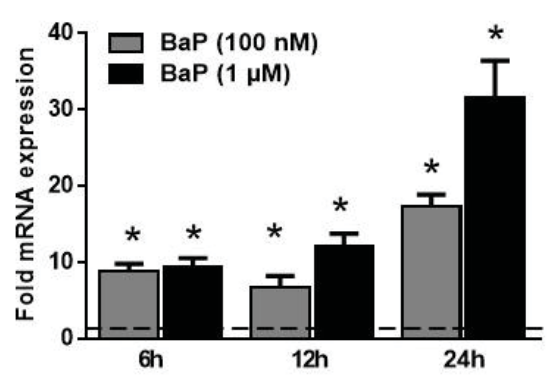

CYP1A1 GAPDH
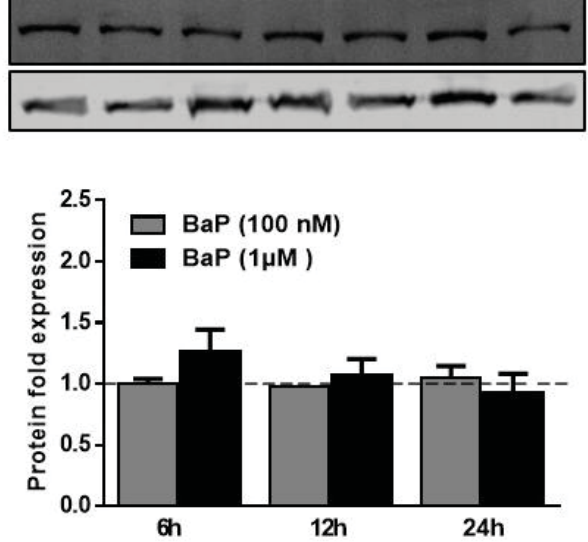

B
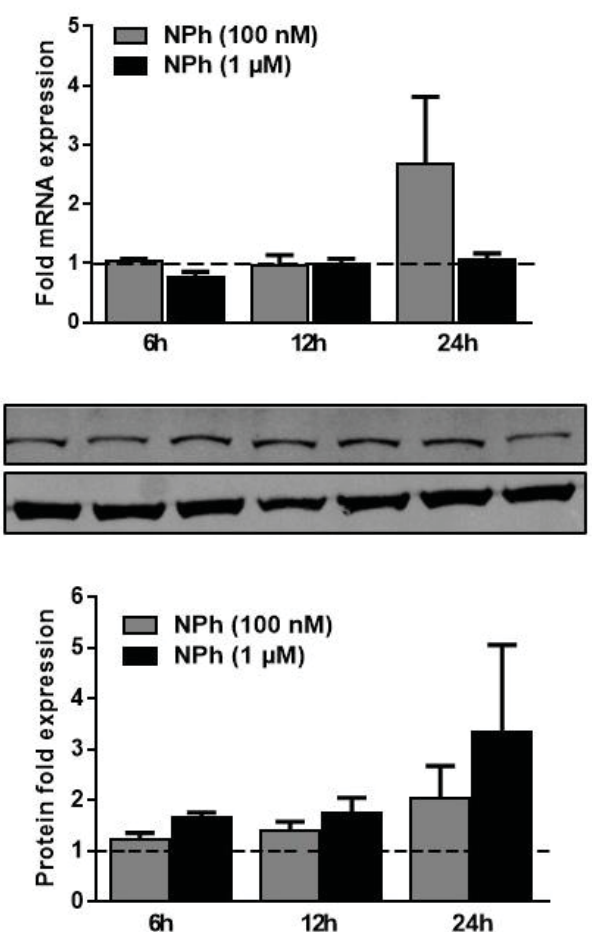

C
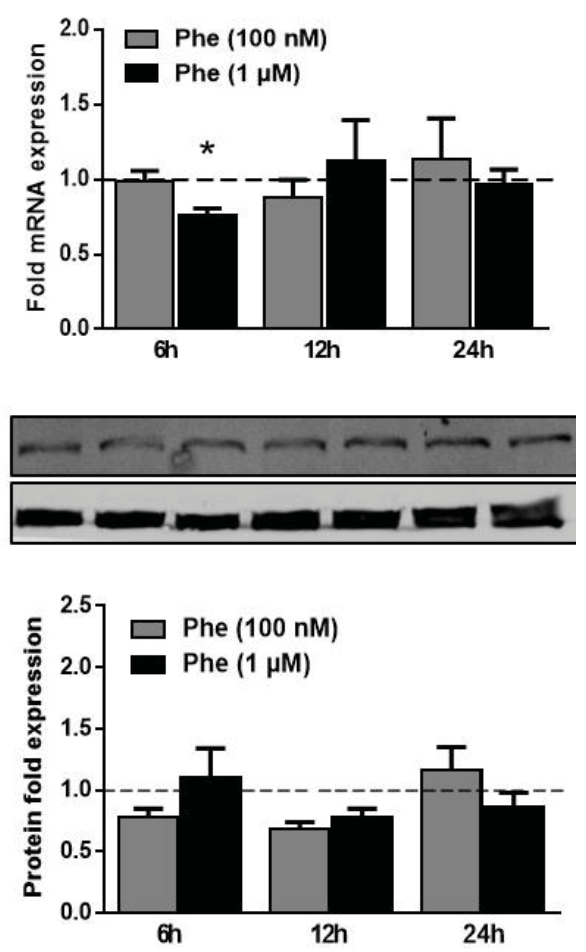

Figure 4-2. Effect of acute treatment of BaP (A), NPh (B), and Phe (C) on mRNA and protein expression of CYP1A1 in $\mathbf{U} 937$ cells.

The U937 cells were treated with $100 \mathrm{nM}$ and $1 \mu \mathrm{M}$ of $\mathrm{BaP}, \mathrm{NPh}$, and Phe for 6,12 and 24 hours. The mRNA fold expressions were calculated using qRT-PCR and the protein fold expressions were measured by Western blots, and normalized with control (DMSO treated cells) whose expression was set at 1-fold. GAPDH was used as an endogenous control. Blots are representative of at least three independent experiments. The data are presented as a mean $\pm \mathrm{SEM}$ of three independent experiments. * represents $\mathrm{p} \leq 0.05$, compared with the control group. 


\section{CYP3A4}

A

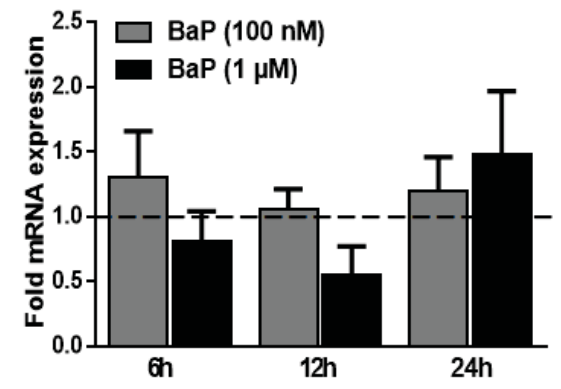

CYP3A4 GAPDH

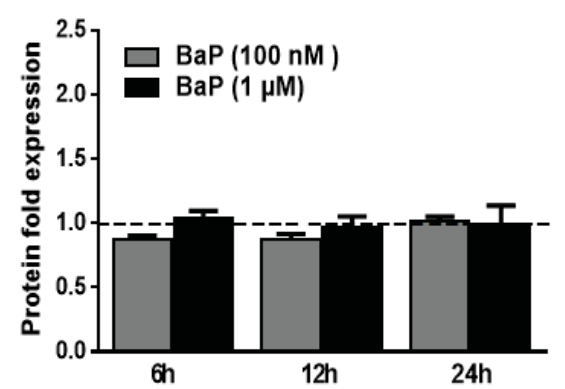

B
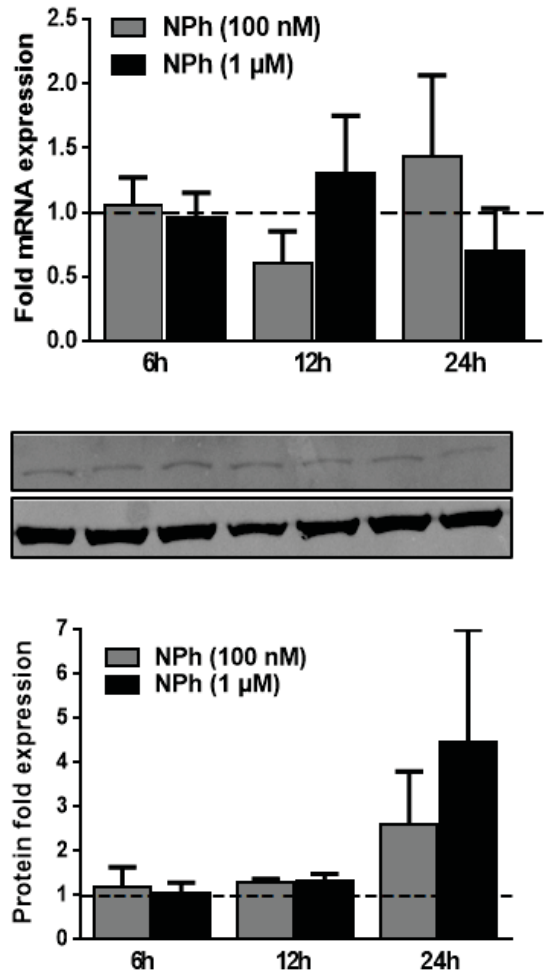

C
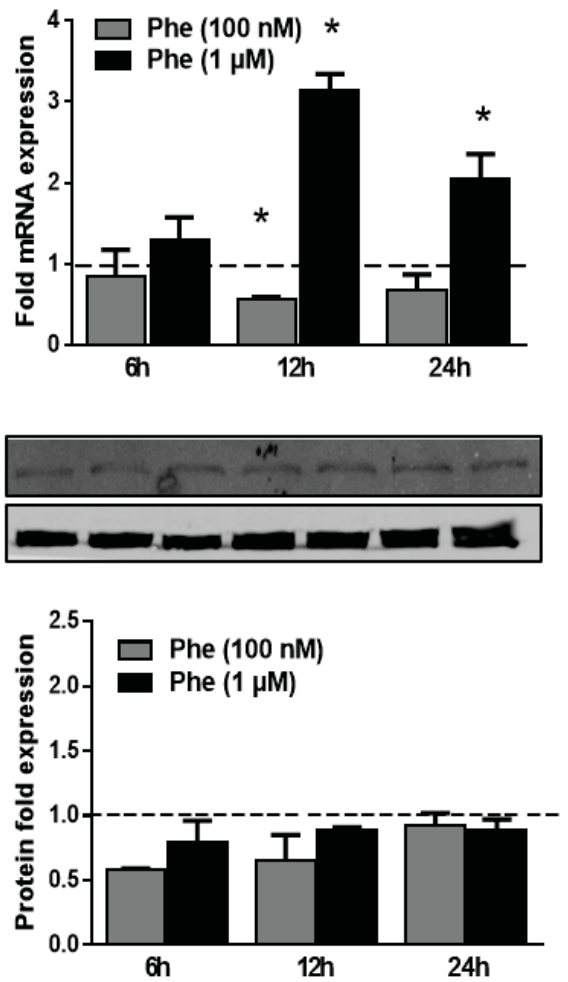

Figure 4-3. Effect of acute treatment of BaP (A), NPh (B), and Phe (C) on mRNA and protein expression of CYP3A4 in $\mathbf{U 9 3 7}$ cells.

The U937 cells were treated with $100 \mathrm{nM}$ and $1 \mu \mathrm{M}$ of BaP, NPh, and Phe for 6, 12 and 24 hours. The mRNA fold expressions were calculated using qRT-PCR and the protein fold expressions were quantified by Western blots, and normalized with control (DMSO treated cells) whose expression was set at 1-fold. GAPDH was used as an endogenous control. Blots are representative of at least three independent experiments. The data are presented as a mean \pm SEM of three independent experiments. * represents $\mathrm{p} \leq 0.05$, compared with the control group. 


\section{SOD1}

A

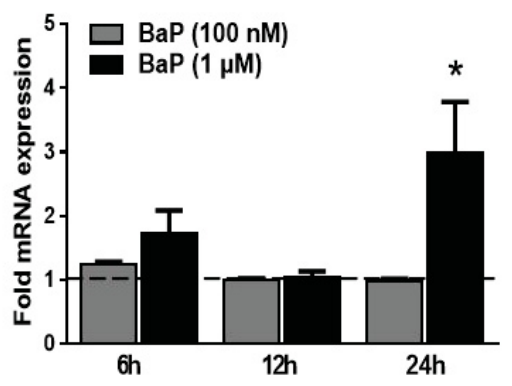

SOD1 GAPDH
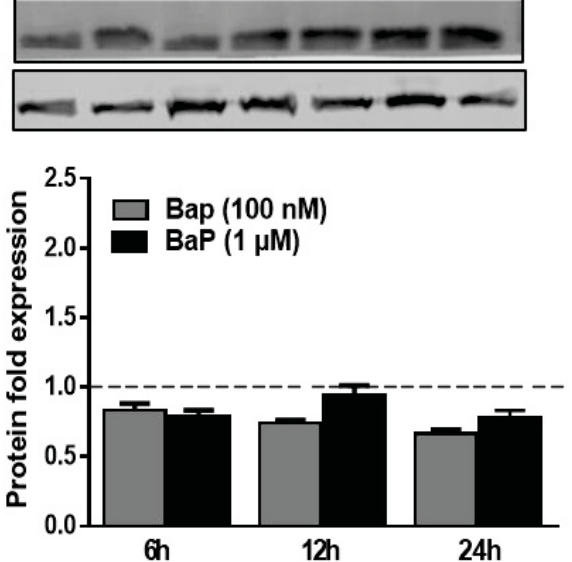

B
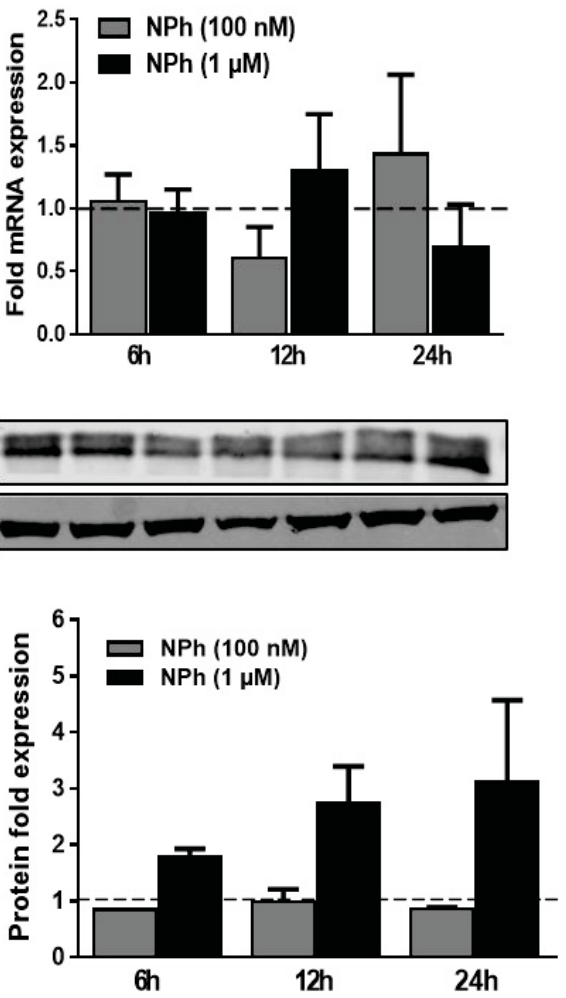

C
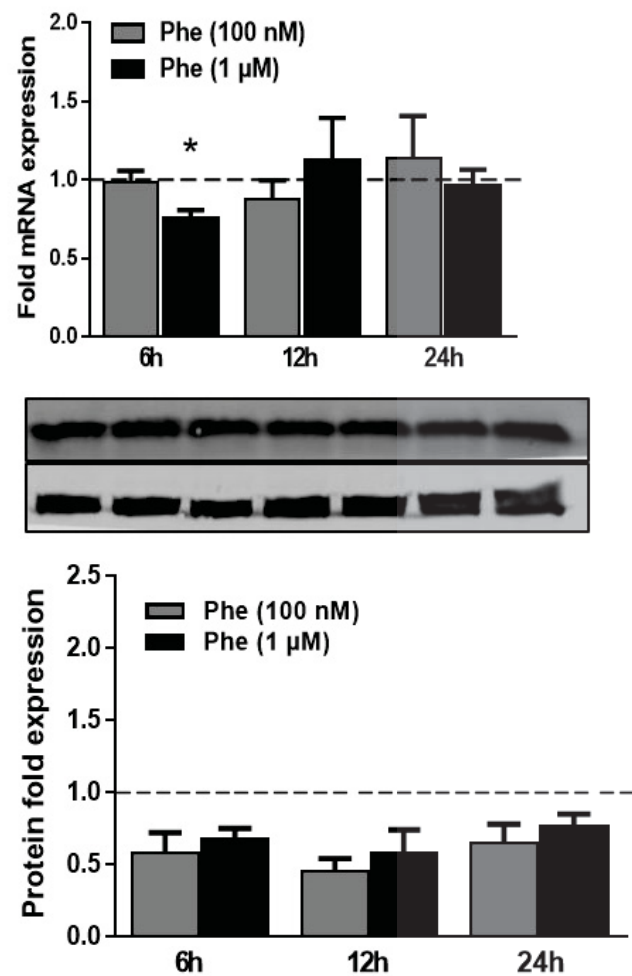

Figure 4-4. Effect of acute treatment of BaP (A), NPh (B), and Phe (C) on mRNA and protein expression of SOD1 in U937 cells.

The U937 cells were treated with $100 \mathrm{nM}$ and $1 \mu \mathrm{M}$ of $\mathrm{BaP}, \mathrm{NPh}$, and Phe for 6,12 and 24 hours. The mRNA fold expressions were calculated using qRT-PCR and the protein fold expressions were quantified by Western blots, and normalized with control (DMSO treated cells) whose expression was set at 1-fold. GAPDH was used as an endogenous control. Blots are representative of at least three independent experiments. The data are presented as a mean \pm SEM of three independent experiments. * represents $\mathrm{p} \leq 0.05$, compared with the control group. 
Catalase

A

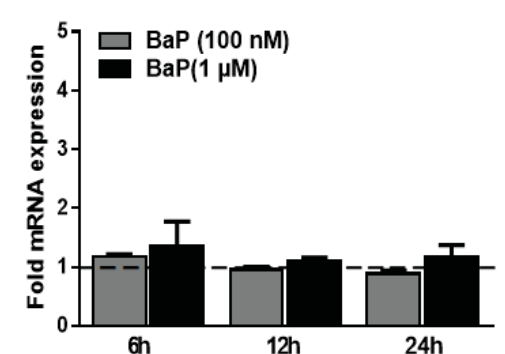

Catalase GAPDH
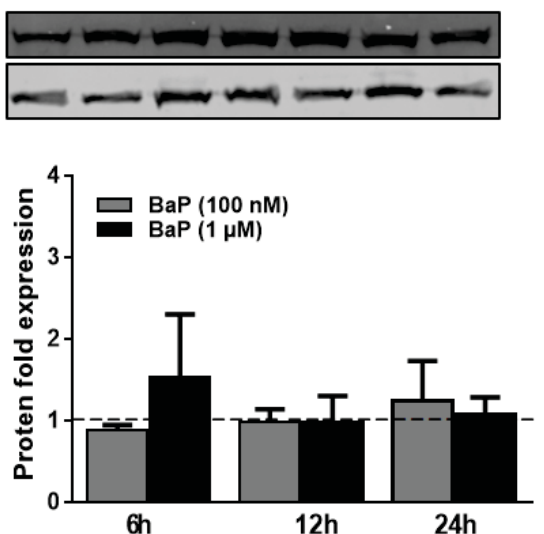

B
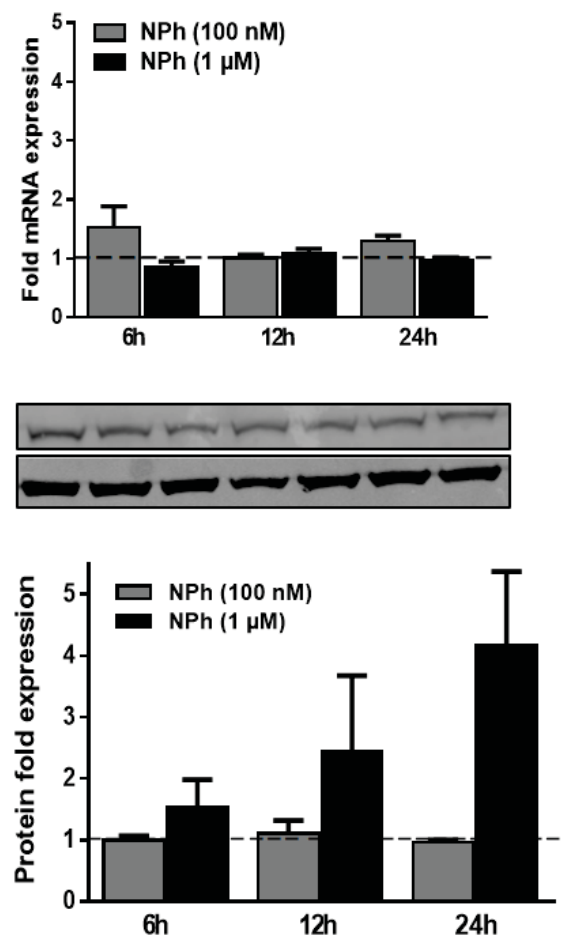

$\mathrm{C}$
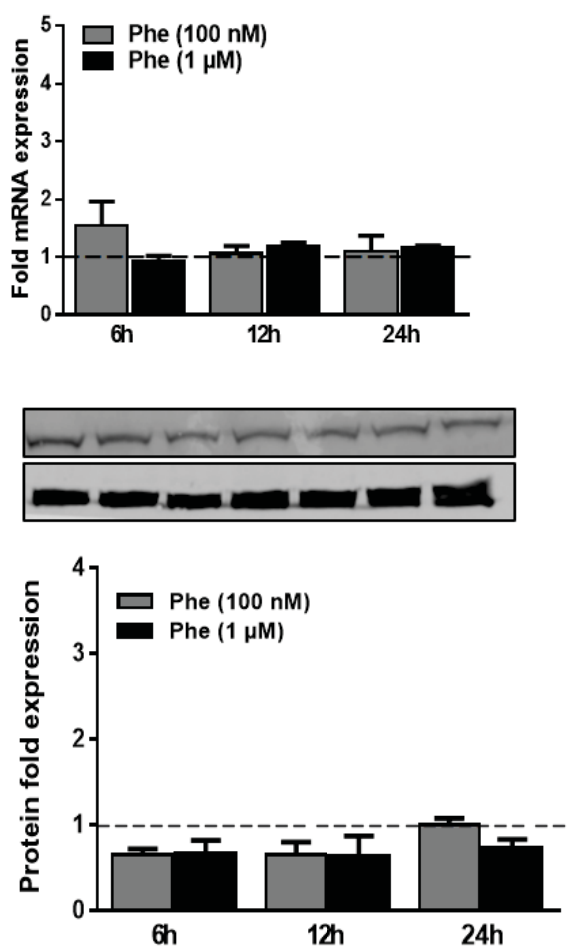

Figure 4-5. Effect of acute treatment of $\mathrm{BaP}(\mathrm{A}), \mathrm{NPh}(\mathrm{B})$, and Phe (C) on mRNA and protein expression of catalase in $\mathbf{U} 937$ cells.

The U937 cells were treated with $100 \mathrm{nM}$ and $1 \mu \mathrm{M}$ of BaP, NPh, and Phe for 6,12 and 24 hours. The mRNA fold expressions were calculated using qRT-PCR and the protein fold expressions were quantified by Western blots, and normalized with control (DMSO treated cells) whose expression was set at 1-fold. GAPDH was used as an endogenous control. Blots are representative of at least three independent experiments. The data are presented as a mean $\pm \mathrm{SEM}$ of three independent experiments. * represents $\mathrm{p} \leq 0.05$, compared with the control 
increase in ROS was observed with the chronic treatment of $25 \mathrm{nM} \mathrm{BaP}(\sim 1.5$ fold, $\mathrm{p} \leq 0.05)$ and $100 \mathrm{nM} \mathrm{BaP}(\sim 2.5$ fold, $\mathrm{p} \leq 0.05)$ (Figure 4-6A-B). However, there was no significant alteration in the ROS levels with $5 \mathrm{nM}$ BaP. Similarly, no significant change in ROS was observed with $\mathrm{NPh}$ and Phe treatments. A statistically significant $(\mathrm{p} \leq 0.05)$ decrease in cell viability $(\sim 60 \%)$ was observed with $100 \mathrm{nM}$ BaP treatment. Lower concentrations ( $5 \mathrm{nM}$ and $25 \mathrm{nM}$ ) of $\mathrm{BaP}$, as well as, the $100 \mathrm{nM} \mathrm{NPh}$ and Phe treatments, however, did not have any effect on cell toxicity (Figure 4-7B). Similar decrease in cell viability with $100 \mathrm{nM} \mathrm{BaP}$ treatment was observed using XTT cell viability assay ( $\sim 40 \%$ decrease in cell viability, Figure 4-7C). Figure 4-7A show the graphical representation of cell viability, measured via flow cytometry.

\section{Effect of Chronic Treatment of BaP, NPh, and Phe on Caspase-3 Activity}

In order to delineate the mechanism of cytotoxicity after chronic treatment of PAHs (BaP, NPh, and Phe), we monitored the caspase-3 activity. We observed a significant increase in caspase- 3 activity with $100 \mathrm{nM} \mathrm{BaP}$ treatment ( $\sim 2$ fold, $\mathrm{p} \leq 0.05$, Figure 4-6C). However, there was no effect on caspase-3 activity with lesser concentrations of $\mathrm{BaP}(5 \mathrm{nM}$ and $25 \mathrm{nM})$ or $100 \mathrm{nM}$ of $\mathrm{NPh}$ or Phe.

\section{Effect of Chronic Treatment of BaP, NPh, and Phe on the Expression of CYPs at mRNA and Protein Levels}

PAHs are metabolized by various CYP enzymes, mainly CYPs 1A1, 1B1, and to a lesser extent by CYPs 1A2, 2C9, 3A4, and 2C19 into reactive metabolites and produce ROS that cause DNA damage [232]. Therefore, to examine the effect of these compounds on expressions of CYPs 1A1 and 3A4 that are prominently present in U937 cells, we treated the cells separately with $100 \mathrm{nM}$ of each compound for seven days and measured the mRNA and protein levels of CYP1A1 and CYP3A4 (Figure 4-8). With BaP treatment, we observed $\sim 15$ folds increase $(\mathrm{p} \leq 0.05)$ in the CYP1A1 mRNA expression (Figure 4-8A) and $\sim 3$ folds increase in CYP3A4 mRNA expression (Figure 4-8B). However, the CYP1A1 and 3A4 protein expression levels quantified from the Western blots did not correspond to the respective changes in mRNA expression levels. NPh and Phe did not show much change in the induction of either CYPs. Interestingly, there was $\sim 2.5$ fold $(\mathrm{p} \leq 0.05)$ increase in the protein expression of CYP1A1 in the cells treated with $\mathrm{NPh}$ (Figure 4-8A, lower panel).

\section{Effect of Chronic Treatment of BaP, NPh, and Phe on the Expression of AOEs at the mRNA and Protein Levels}

PAHs are likely to induce expression of AOEs to combat PAH-induced oxidative stress in U937 cells. To determine whether these compounds alter the expression of AOEs, we measured the mRNA and protein levels of the major AOEs SOD1 and catalase (Figure 4-9). Chronic (seven days) exposure of $100 \mathrm{nM} \mathrm{BaP}$ upregulated the mRNA 


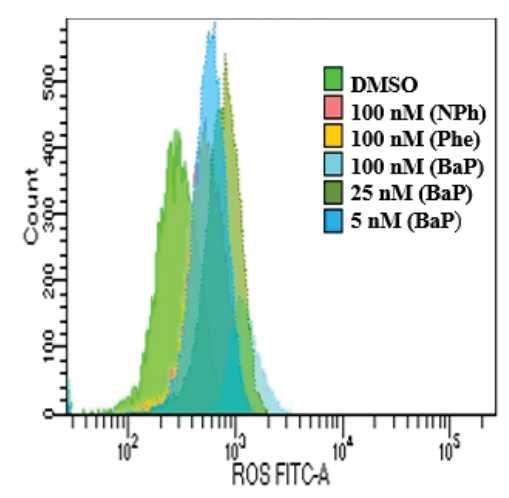

A

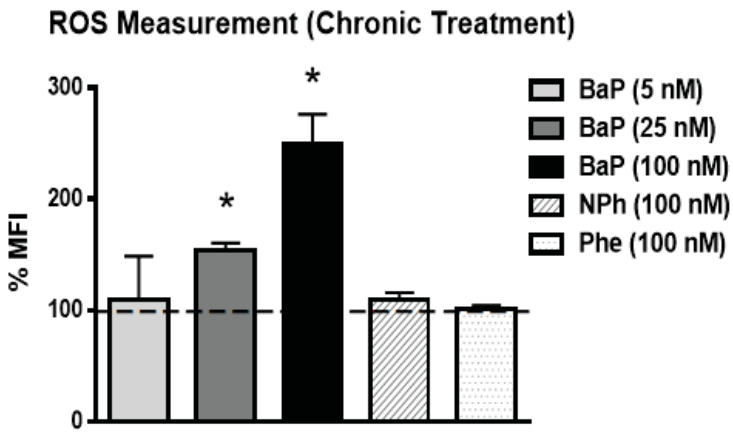

B
Caspase-3 Activity (Chronic Treatment)

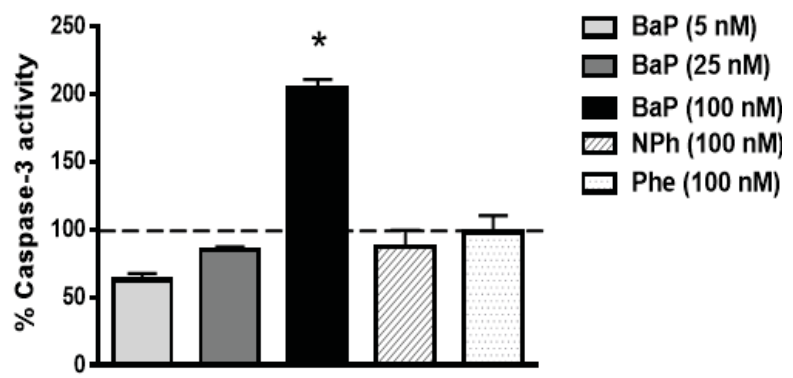

C

Figure 4-6. Effect of chronic (7 days) treatment of BaP, NPh, and Phe on reactive oxygen species (ROS) level (A-B) and caspase- 3 activity $(C)$ in $U 937$ cells.

The U937 cells were treated with $(5,25$ and 100) nM BaP, $100 \mathrm{nM} \mathrm{NPh}$, and $100 \mathrm{nM}$ Phe for seven days. ROS level and caspase- 3 activity were calculated and normalized with control that was set as $100 \%$. The data is presented as mean \pm SEM of the three independent experiments. * represents $\mathrm{p} \leq 0.05$, compared with the control group. 

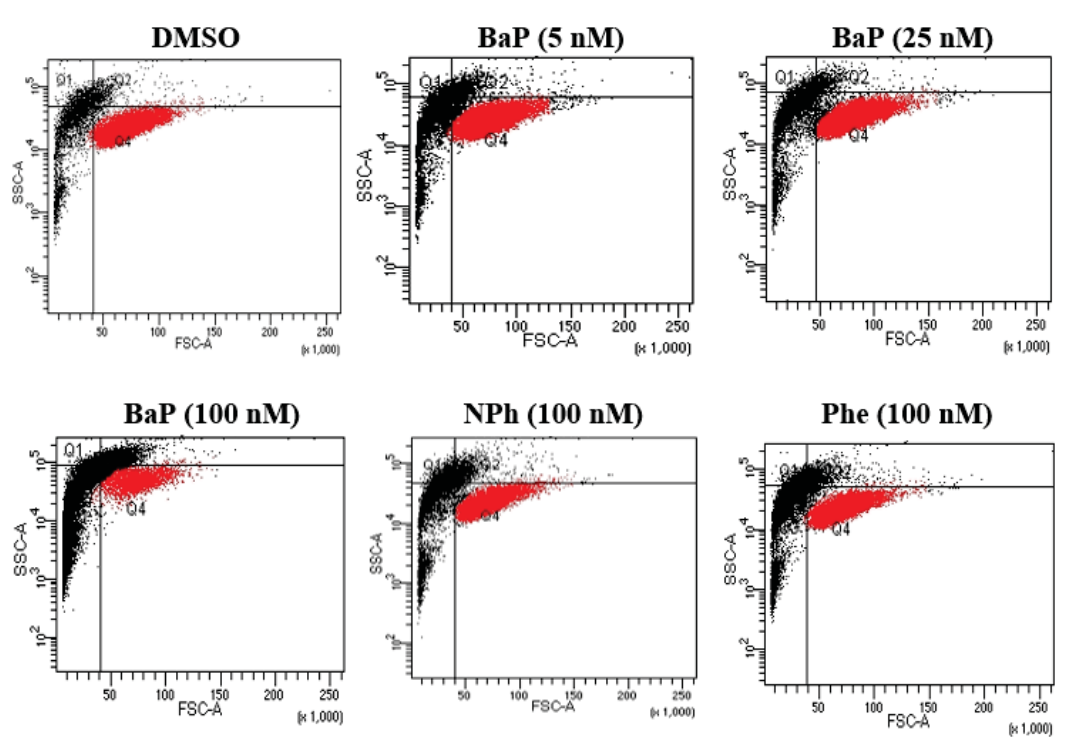

B

Cell Viability by Flow Cytometry

(Chronic Treatment)

$\square \operatorname{BaP}(5 \mathrm{nM})$

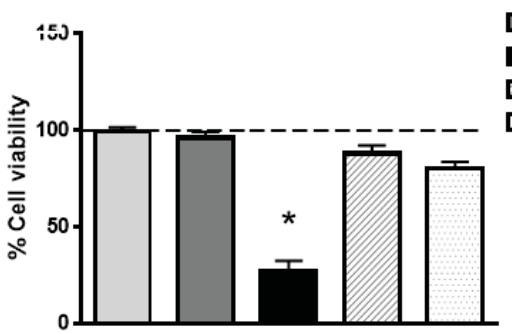

C

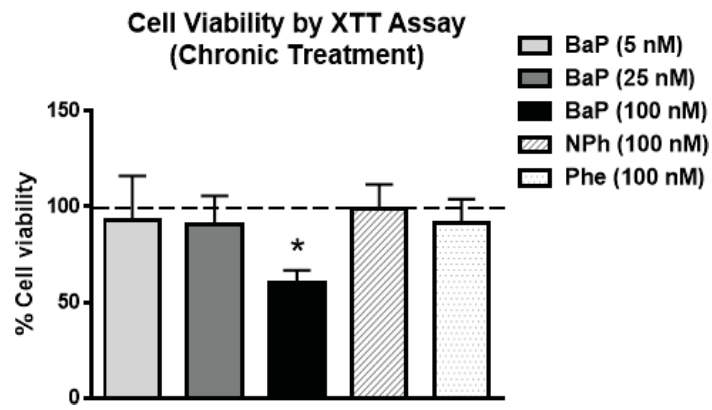

Figure 4-7. Cell viability upon chronic treatment by BaP, NPh, and Phe measured by flow cytometry (A-B) and XTT assay (C).

The U937 cells were treated with $(5,25$ and 100) $\mathrm{nM} \mathrm{BaP,} 100 \mathrm{nM} \mathrm{NPh}$, and $100 \mathrm{nM}$ Phe for seven days. Cell viability was calculated using flow cytometry and XTT assay, and normalized with control that was set as $100 \%$. The data is presented as mean \pm SEM of the three independent experiments. * Represents $\mathrm{p} \leq 0.05$, compared with the control group. 
A
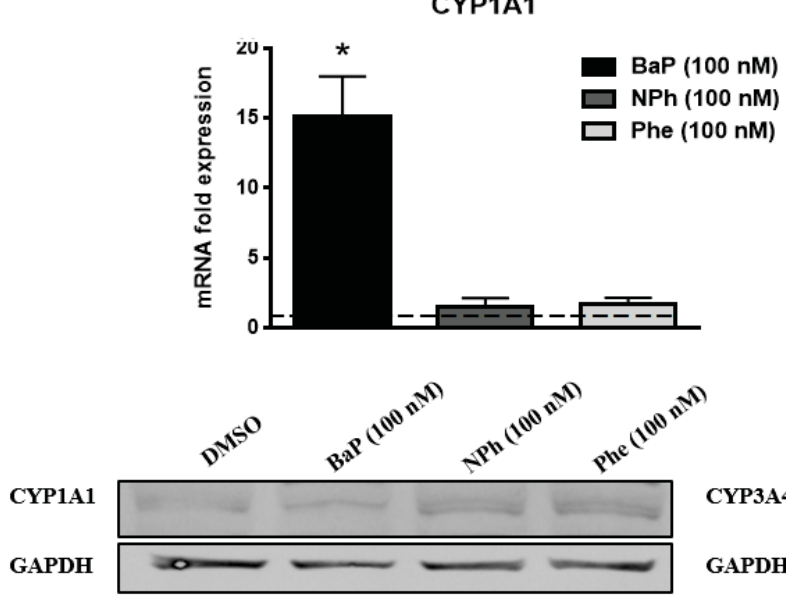

CYP1A1

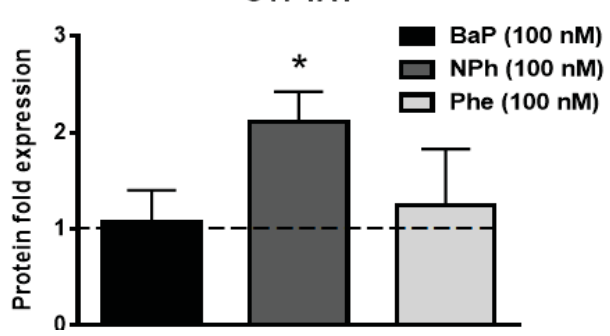

CYP3A4
B

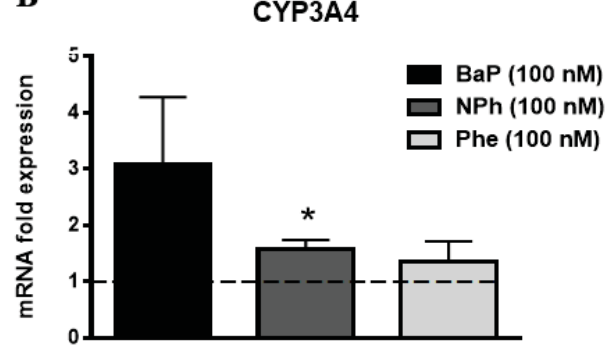

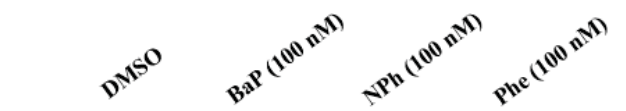

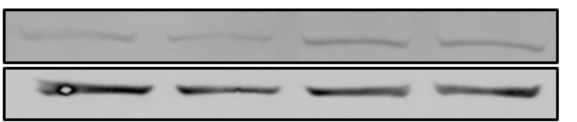

CYP3A4

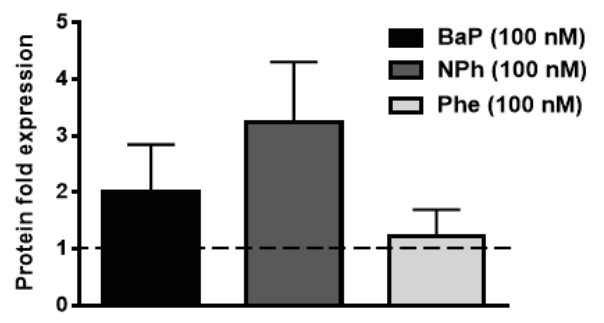

Figure 4-8. Effect of chronic (7 days) treatment of BaP, NPh, and Phe on mRNA and protein expression of CYP1A1 (A) and CYP3A4 (B) in U937 cells.

The U937 cells were treated with $100 \mathrm{nM} \mathrm{BaP,} 100 \mathrm{nM} \mathrm{NPh}$, and $100 \mathrm{nM}$ Phe for seven days. The mRNA fold expressions were calculated using qRT-PCR and normalized with control (DMSO treated cells) whose expression was set at 1-fold. GAPDH was used as an endogenous control. The protein fold expressions were quantified by Western blot and normalized with control that was set to 1-fold at every time point. Blots are representative of at least three independent experiments. The data are presented as a mean $\pm \mathrm{SEM}$ of three independent experiments. *represents $\mathrm{p} \leq 0.05$, compared with the control group. 

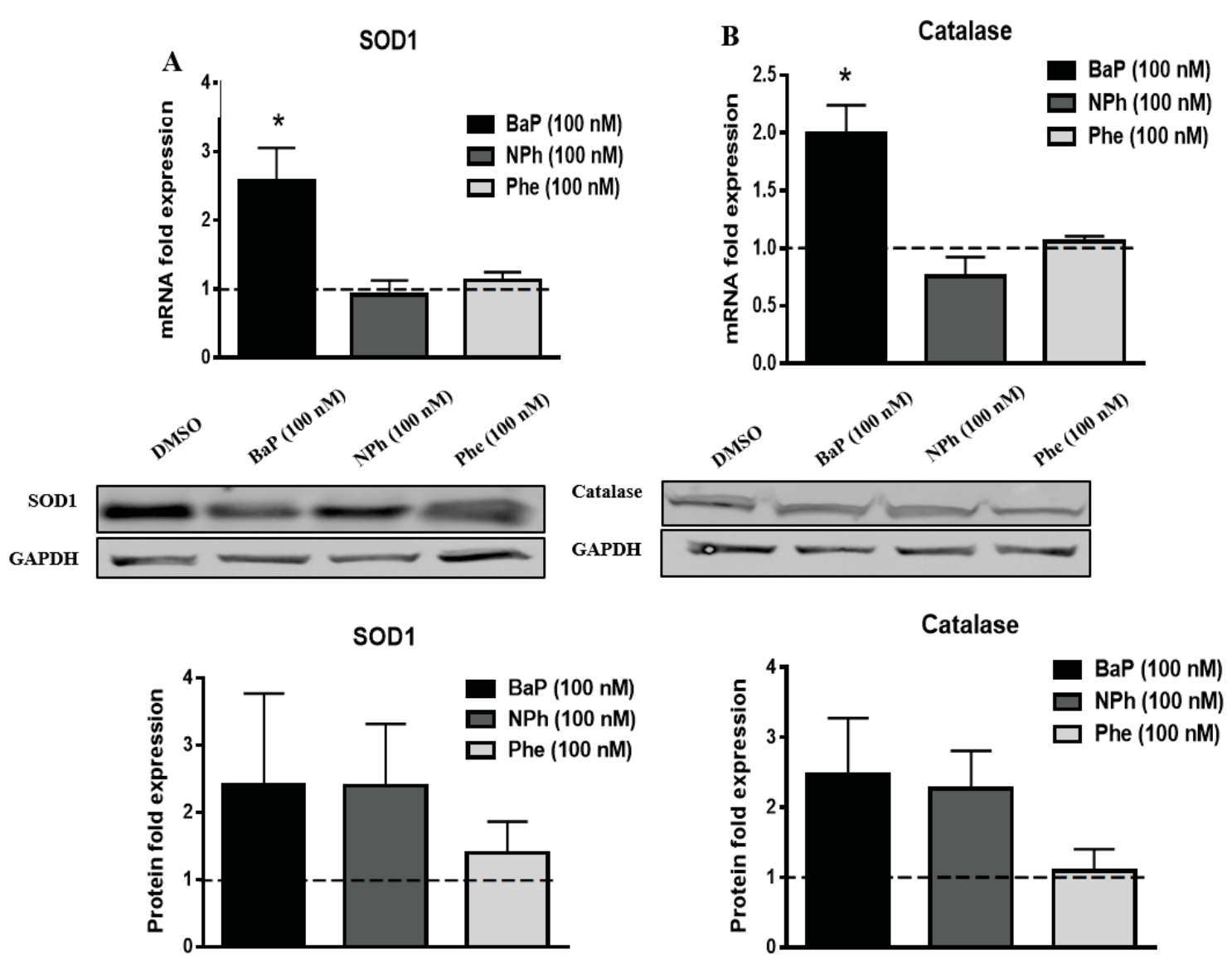

Figure 4-9. Effect of chronic (7 days) treatment of BaP, NPh, and Phe on mRNA and protein expression of AOEs SOD1 (A) and catalase (B) in U937 cells.

The U937 cells were treated with $100 \mathrm{nM} \mathrm{BaP,} 100 \mathrm{nM} \mathrm{NPh}$, and $100 \mathrm{nM}$ Phe for seven days. The mRNA fold expressions were calculated using qRT-PCR and normalized with control (DMSO treated cells) whose expression was set at 1-fold. GAPDH was used as an endogenous control. The protein fold expressions were quantified by Western blot and normalized with control (DMSO treated cells) whose expression was set at 1 fold. Blots are representative of at least three independent experiments. The data are presented as a mean \pm SEM of three independent experiments. *represents $\mathrm{p} \leq 0.05$, compared with the control group. 
expression of both SOD1 ( 2.5 folds, $p \leq 0.05$, Figure 4-9A) and catalase ( $\sim 2$ folds, $\mathrm{p} \leq 0.05$, Figure 4-9B). The corresponding protein expression levels of both AOEs were also upregulated by similar proportions: SOD1 ( $\sim 2.5$ fold $)$ and catalase $(\sim 2.5$ fold $)$ but the data were not statistically significant (Figure 4-9A-B, lower panel). Further, treatment with NPh or Phe did not significantly alter the mRNA or protein expression of AOEs.

\section{Effect of Chronic Treatment of BeA and BeF on ROS, Cell Viability, and Caspase-3 Activity}

In addition to three PAHs (BaP, NPh, and Phe), BeA and $\mathrm{BeF}$ are other PAHs that have carcinogenic potential. Therefore, we also studied these compounds with regard to oxidative stress and cytotoxicity. The cells were treated with $100 \mathrm{nM}$ of BeA or BeF for seven days and were examined for ROS level and cell viability. Cell viability data from XTT assay showed approximately $10-13 \%$ cell death when treated with $100 \mathrm{nM}$ of BeA or BeF for seven days (Figure 4-10A). A significant decrease in ROS level was observed with the chronic treatment of BeA $100 \mathrm{nM}(\sim 35 \%, \mathrm{p} \leq 0.05)$ as well as BeF $100 \mathrm{nM}$ $(\sim 23 \%, p \leq 0.05)$ (Figure 4-10B). There was no significant change in the caspase-3 activity with chronic treatment of both the compounds (Figure 4-10C). Overall, treatment with $\mathrm{BeA}$ and $\mathrm{BeF}$ had relatively less effect on the oxidative stress and cell viability of $\mathrm{U} 937$ compared to $\mathrm{BaP}$.

\section{Effect of Chronic Treatment of BaP on CYP Expression, ROS and Cell Viability in Primary Macrophages}

Of the five compounds, only exposure with $\mathrm{BaP}(100 \mathrm{nM})$ resulted in significant increased expression of CYPs and AOEs, ROS and cell death in U937 cells. So, we exposed the primary macrophages with $100 \mathrm{nM} \mathrm{BaP}$ to verify our observations in U937 cells. When we treated the primary macrophages with BaP $100 \mathrm{nM}$ for seven days, we observed $\sim 25 \%$ cell death accompanied by $\sim 30 \%$ increase in ROS and $\sim 10$ and $\sim 3$ fold $(\mathrm{p} \leq 0.05)$ increase in CYP 1A1 and 3A4 respectively (Figure 4-11A-D). These results confirm that $\mathrm{BaP}$ exposure leads to cytotoxicity in monocytes/macrophages via CYPmediated oxidative stress pathway.

\section{Discussion}

Carcinogenic effects of smoking have been widely studied in various organs, especially lungs and liver [233, 234]. Several studies have also reported CYP-mediated PAH toxicity in different organs $[26,27]$. However, relatively less information is available on the effects of smoking constituents on the blood cells such as monocytes and lymphocytes. A recent study conducted in our lab has shown that nicotine causes oxidative stress in monocytic cells U937 cells through a CYP-mediated pathway [230]. 

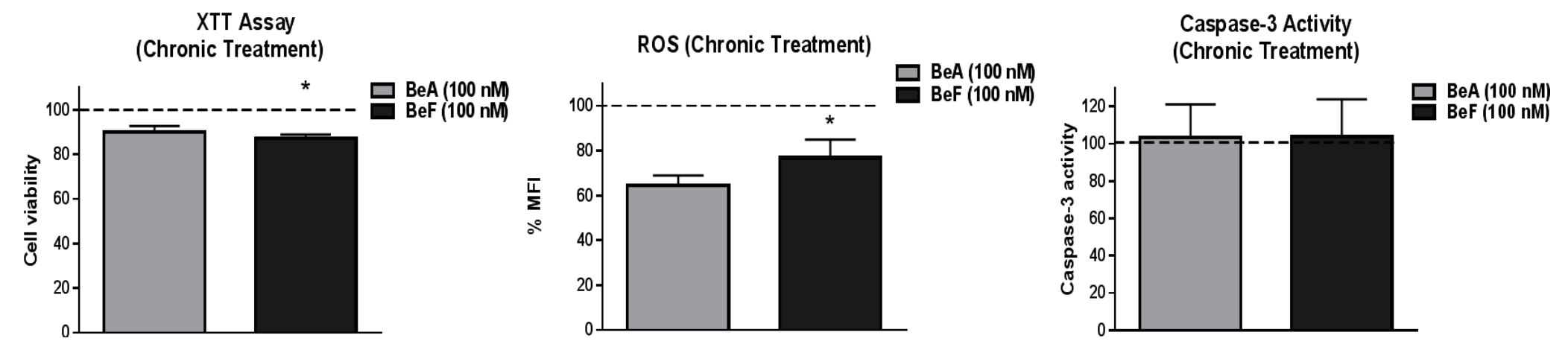

Figure 4-10. Cell viability (A), ROS level (B) and caspase-3 activity (C) upon chronic treatment by BeA and BeF in U937 cells.

The U937 cells were treated with $100 \mathrm{nM}$ BeA and $100 \mathrm{nM}$ BeF for seven days. ROS level, cell viability, and caspase-3 activity were measured and normalized with control that was set as $100 \%$. The data is presented as mean \pm SEM of the three independent experiments. * represents $\mathrm{p} \leq 0.05$, compared with the control group. 

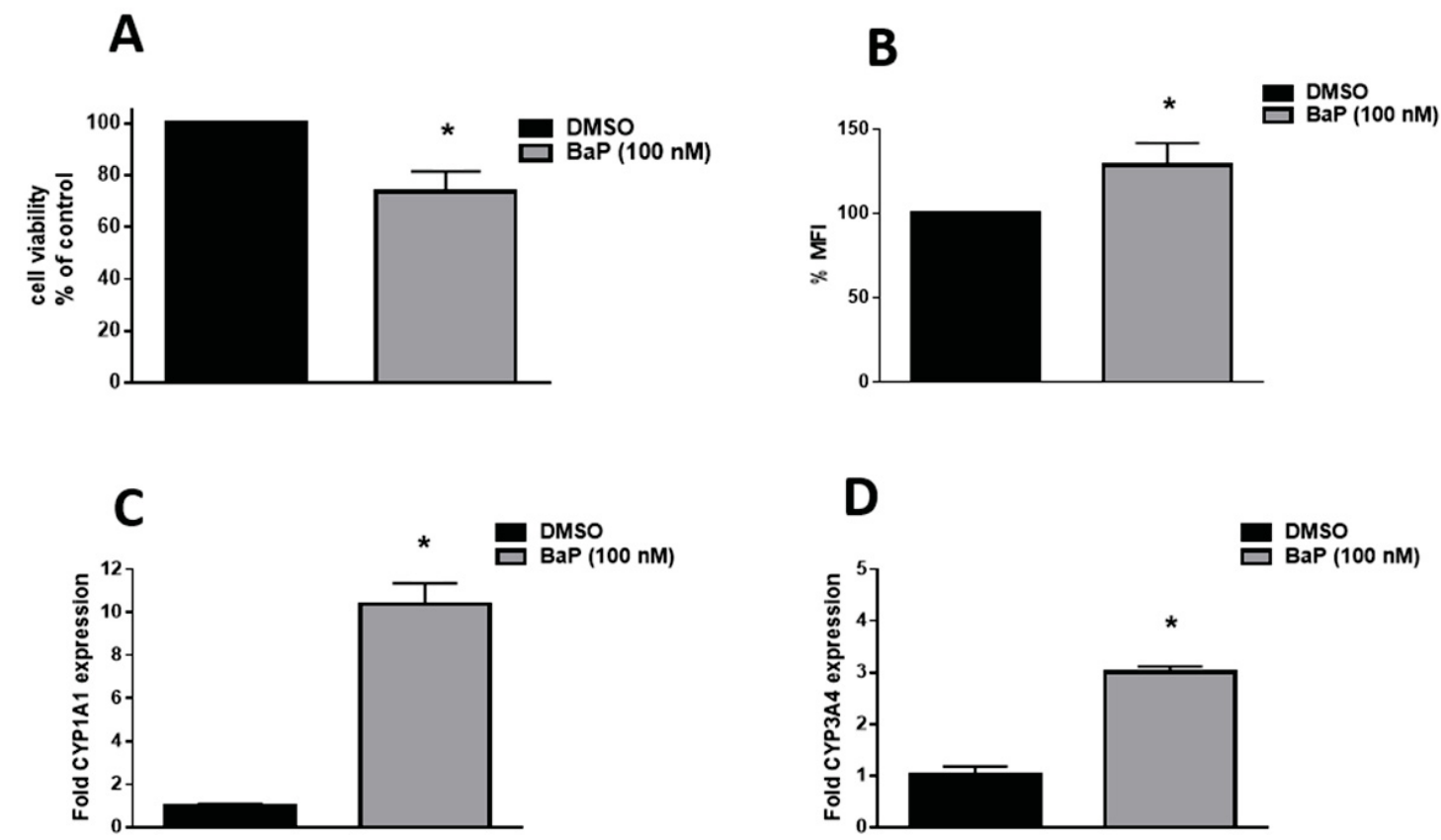

Figure 4-11. Cell viability (A), ROS level (B) and mRNA expression level of CYP1A1 (C) and CYP3A4 (D) upon chronic treatment by BaP in primary macrophages.

The differentiated macrophages obtained from healthy donors were treated with $\mathrm{BaP}$ (100 $\mathrm{nM}$ ) for 7 days. Cell viability and ROS were calculated and normalized with control that was set as $100 \%$. The mRNA expression of treated cells were normalized with control (DMSO treated cells) whose expression was set at 1 -fold. The results for cell viability were confirmed using 3 different donors. Due to inadequate number of primary cells, ROS and CYPs expression level were examined in only one donor. The data is presented as mean $\pm \mathrm{SEM}$ of the three replicates for ROS and two replicates for CYP expression levels. * represents $\mathrm{p} \leq 0.05$, compared with the control group. 
However, there is no report on the effect of PAHs and relative contribution of different PAHs on oxidative stress and cytotoxicity, and underlying mechanism in the monocytic cells. The present study is the first report of relative contribution of five PAHs (BaP, $\mathrm{NPh}, \mathrm{Phe}, \mathrm{BeA}$, and $\mathrm{BeF}$ ) on the expression of CYPs and AOEs, induction of ROS, and cytotoxicity in U937 monocytic cells. The results from this study support the existing literatures that among the tested PAHs, $\mathrm{BaP}$ is the most harmful compound, which causes oxidative stress and subsequent cytotoxicity, at least in part through CYP pathway.

Zhu et al. (2014) have shown that $\mathrm{BaP}(5 \mu \mathrm{M})$ significantly increases the expressions of CYP1A1 and CYP1B1, ROS level, and cytotoxicity in lung epithelial cells (BEAS-2B cells) after 24 hour treatment [235]. In this paper, we examined the effect of both acute (6-24 hours) and chronic (7 days) exposure of PAHs in U937 cells. We initially treated the cells with $100 \mathrm{nM}$ and $1 \mu \mathrm{M}$ of each of the PAHs for acute study. These concentrations are very near to the physiological concentrations of PAHs that caused toxicity in different cell lines [235, 236]. For the chronic treatment, the lower concentration $(100 \mathrm{nM})$ was preferred because higher concentration $(1 \mu \mathrm{M})$ resulted in immense cell death when exposed for a prolonged period. There are very few reports that account for the toxicity of chronic BaP exposure in human cells. The reports of chronic $\mathrm{BaP}$ exposure in animal model suggest its association with neurotoxicity [237], DNA damage [238], carcinogenicity, and cytotoxicity [239]. Most of the studies of BaP toxicity have been conducted at higher concentration for an acute period. In this context, our experimental design to study the acute as well as chronic effects of near physiological concentrations of PAHs on monocytic cells is pragmatic because it closely simulates the effects of tobacco on these cells in vivo.

We have used U937 cells derived from histiocytic lymphoma tissues, which have functionally deficient p53 tumor suppressor gene due to gene mutation [240]. The phenomenon of apoptosis is apparently ceased in these cell lines, causing massive cell proliferation. However, apoptosis may occur in U937 cells when triggered by various stress factors such as ROS, via a p53-independent mechanism. There are also reports that associate $\mathrm{p} 53$ with $\mathrm{BaP}$-induced CYP1A1 expression, which occur via $\mathrm{p} 53$ binding to a $\mathrm{p} 53 \mathrm{RE}$ in the CYP1A1 regulatory region [241]. Some studies suggest that p53 upregulates the gene associated with antioxidant activity and thereby prevent the genome from oxidative damage by ROS [242]. Due to the lack of functional p53 in U937 cells, induction of CYP1A1 and antioxidants by BaP occur via a p53 independent pathway, probably via Nf-kB-mediated pathway. Another possible pathway for induction of CYPs could be via binding of $\mathrm{BaP}$ to arylhydrocarbon receptor (AhR) and the subsequent gene activation by constitution of the Arnt protein-BaP complex with XRE responsive element [243].

With acute treatment of three $\mathrm{PAHs} \mathrm{BaP}, \mathrm{NPh}$, and Phe, we did not observe any significant change in the expressions of CYPs and AOEs, ROS level, or cell viability. So, we exposed the cells for seven days with $100 \mathrm{nM}$ of each of the compounds to monitor their chronic effect. Out of five PAHs (BaP, NPh, Phe, BeA, and BeF), only BaP (100 $\mathrm{nM}$ ) significantly increased the expressions of CYPs and AOEs, generation of ROS, and cytotoxicity in $\mathrm{U} 937$ cells. The prolonged exposure of $\mathrm{BaP}$ to the cells probably caused 
the accumulation of ROS, via CYP-pathway. Elevated ROS then contributed to the oxidative stress and cytotoxicity. An increased level of AOE expression with chronic BaP treatment is also consistent with the finding that there was an increase in the ROS level.

$\mathrm{BaP}$ is considered a prototype compound for PAH carcinogenicity. IARC has listed $\mathrm{BaP}$ as a group I human carcinogen, while $\mathrm{NPh}$, Phe, $\mathrm{BeA}$, and $\mathrm{BeF}$ have been classified as compounds possibly carcinogenic to humans (IARC 2004). BaP-induced carcinogenesis has been studied extensively for more than five decades. Previous studies reveal that CYP metabolites of $\mathrm{BaP}$ form DNA adducts that inactivate the tumor suppressor p53 in human epithelial cells and bronchial epithelial cells [233]. Kucab et al. (2015) has also suggested the role of $\mathrm{BaP}$ and its metabolites in p53 mutagenesis, which is a common pathway observed in almost half of human cancers [244]. However, the role of $\mathrm{BaP}$ and other PAHs with respect to oxidative stress and cytotoxicity in monocytic cells is not known. In this context, evaluation of the role of PAHs and their relative contributions to the expressions of CYPs, induction of oxidative stress, and cytotoxicity further validates the literature that $\mathrm{BaP}$ is toxic to many cells including monocytic cells.

BaP induces the expression of several CYPs including 1A1 and 1B1 in different cells [245-247]. Since the basal expression of CYP1B1 in U937 cells is very low [248], we examined the effect of PAHs on the expression of CYP1A1 only. Cytotoxicity induced by CYP1A1 metabolites of $\mathrm{BaP}$ has been reported in different cellular systems: lung cells [249], porcine urinary bladder epithelial cells [250], and bone marrow cells [251]. CYP1A1 metabolizes BaP into BPDE which forms DNA adducts leading to genotoxicity and carcinogenesis. Some studies also suggest that ROS generated from BaP via CYP pathway causes oxidative stress that lead to cytotoxicity. In the present study, CYP1A1 mRNA expression was consistently upregulated ( $\sim 15$ fold) following the acute and chronic treatment of $\mathrm{BaP}(100 \mathrm{nM})$ in U937 cells. However, the CYP1A1 protein levels were not consistent with the mRNA expression levels. Similar contradiction was reported in earlier studies [252], the reason for which is not clear. However, it is possible that CYP1A1 is relatively less stable upon extraction or there may be post-translational modification of the protein. The metabolites obtained from the CYP1A1-mediated oxidation of BaP may be responsible for the elevated ROS level and subsequent oxidative stress and cytotoxicity. Further investigation is required to confirm our hypothesis that CYP1A1 metabolizes BaP into ROS-generating metabolites that cause cytotoxicity in U937 cells. This can be done either by knocking down CYP1A1 gene or by using a selective inhibitor of CYP1A1 and treating with BaP to determine if ROS generation and cytotoxicity still occur. The chronic exposure to $\mathrm{NPh}(100 \mathrm{nM})$ showed $\sim 2$ fold increase in CY1A1 protein expression but there was no significant effect on AOE expression, ROS, cell viability and caspase- 3 activity. Although, we observed increase in CYP1A1 protein expression to some extent, the induced protein may not have been sufficient enough to metabolize the compound.

CYP3A4 is a major drug metabolizing enzyme that metabolizes about $50 \%$ of the xenobiotics and is present in relatively high abundance compared to other CYPs, not only in hepatic cells, but also in U937 monocytic cells [252]. BaP-induced CYP3A4 upregulation at mRNA level has been observed in HepG2 liver cells and HEK-293 
kidney cells [253]. Kumagai et. al also suggested that BaP enhances CYP3A4 gene expression via PXR activation in liver cells [254]. Furthermore, they suggested the possibility that the metabolites obtained through CYP1A1 metabolism could act as CYP3A4 inducers. It is important to study the expression level of CYP3A4 in context of BaP metabolism and toxicity. CYP3A4 does not metabolize the PAH parent compounds but it does convert dihydrodiols into diol epoxides. However, the rate of conversion is slower than that observed through CYP1A1 metabolism [232]. In the present study, we did not observe any significant change in CYP3A4 expression at mRNA and protein levels after acute or chronic treatment with BaP. Since the overall CYP3A4 expression was low, CYP1A1 metabolites also did not seem to contribute to CYP3A4 induction.

Cells are equipped with an antioxidant defense mechanism to counteract the oxidative stress resulting from elevated ROS [255]. Oxidative stress is alleviated either by endogenous antioxidants such as reduced glutathione (GSH) or by adaptive defense mechanism through induction of genes encoding AOEs [256]. The induction of genes encoding AOEs are regulated by nuclear factor erythroid 2- related factor (Nrf-2) signaling pathway [257]. Any perturbations in the AOE defense system that decrease the AOE expression may aid elevation in ROS level. Higher incidence of oxidative stressinduced carcinogenesis is reported in Nrf-2 knockout mice that are treated with BaP. Furthermore, if the level of ROS exceeds the threshold, the AOEs may not be able to overcome the resulting oxidative insult $[153,258]$. SODs and catalase are major AOEs in majority of the cell lines. In case of acute treatment of $\mathrm{BaP}$, we observed a slight decrease in ROS which may be attributed to the protective activity of the endogenous AOEs. We anticipated higher levels of ROS and AOEs with chronic treatment of the PAHs, but we observed the expected result only with BaP-treated cells. The elevation in gene transcription of SOD1 and catalase over the course of seven-day treatment with BaP may not be sufficient enough to alleviate the high ROS level. Rather, an increase in the expression level of these AOEs are the indication that there is oxidative stress in the system.

In addition to $\mathrm{BaP}-$ mediated mutagenesis and carcinogenesis through DNA adduct formation, there are also reports of apoptosis induced by BaP metabolites in different human cell lines: Daudi B cells [259], H460 lung cancer cells [260], hepatoma HepG2 cells [261] and endometrial cancer RL95-2 cells [262], the latter two being directly associated with CYP-mediated metabolism of BaP. ROS triggers apoptosis by enhancing the permeability of the mitochondrial outer membrane and thereby leaking out pro-apoptotic proteins [263]. Pro-apoptotic proteins aid in the activation of caspases, cysteine proteases that cause cellular degradation. We observed apoptosis in U937 cells via caspase- 3 dependent pathway, with chronic treatment of $\mathrm{BaP}$. Our data suggests the involvement of CYP in the metabolism of $\mathrm{BaP}$ and generation of ROS with chronic treatment of BaP. 


\section{Conclusions}

Therefore, we conclude that BaP causes apoptosis in U937 cells through a caspase-3-dependent pathway. BaP-induced cytotoxicity, in turn, is mediated by ROS that is likely to be generated through CYP-mediated metabolism of BaP. Our previous in vitro and ex vivo studies have suggested the role of nicotine metabolism via CYP2A6 in generating oxidative stress and HIV-1 replication in monocytic cells. The current study has established the fact that, in addition to nicotine, $\mathrm{BaP}$ is the major PAHs that is responsible for inducing oxidative stress, apoptosis, and cytotoxicity, perhaps through the CYP pathway in monocytic cells. The confirmation of increase in CYP expression, ROS generation and cytotoxicity mediated by chronic treatment of $\mathrm{BaP}$ in primary macrophages has further strengthened our findings from U937 monocytic cells. We speculate that BaP-induced oxidative stress via CYP metabolism would enhance HIV-1 replication. In fact, our study with cigarette smoke condensate (CSC), which contains nicotine and PAHs, has demonstrated an increase in HIV-1 replication in primary human monocyte-derived macrophages [153]. This is also based on the fact that $\mathrm{BaP}$ induced greater levels of ROS ( $>150 \%$ ) compared to nicotine (15-20\%) [230]. Taken together these findings indicate that $\mathrm{BaP}$ is likely the most active compound in cigarette smoke, and may generate oxidative stress leading to cytotoxicity and increased HIV-1 replication in monocytic cells. However, this has yet to be fully demonstrated using HIV-infected U937 and human primary monocytic cells. 


\section{CHAPTER 5. BENZO(a)PYRENE ENHANCES HIV-1 REPLICATION IN MONOCYTIC CELLS *}

\section{Introduction}

The association of cigarette smoking and HIV-1 pathogenesis has been demonstrated by multiple studies in the past two decades [153, 154, 157, 159, 264, 265]. Smoking increases HIV-1 infectivity and viral load, and it lowers the CD $4^{+} \mathrm{T}$ cell counts in HIV-1 patients, with a subsequent increase in immunosuppression [135, 153]. Smoking also decreases the response to antiretroviral therapy (ART) by approximately $40 \%$ in HIV-1 patients [160], which further accentuates the hazards of smoking on HIV-1 pathogenesis. However, little is known about the mechanisms underlying smokinginduced HIV-1 replication. A recent study has shown that cigarette smoke condensate (CSC) induces CYP expression and oxidative stress in HIV-1-infected monocyte-derived macrophages, and the findings are consistent with increased oxidative stress, nicotine metabolism and HIV-1 replication in HIV-infected individuals who smoke [154].

Another study has revealed that the toxic metabolites released through the CYP-mediated metabolism of cigarette smoke constituents enhance HIV-1 gene expression through DNA adduct formation [156]. Aqueous tobacco smoke extract is also known to enhance the upregulation of genes that enhance HIV-1 infection, but downregulate the expression of other genes that promote cell survival and antigen presentation [157].

Of the 5300 compounds that are present in CSC, polyaryl hydrocarbons (PAHs) are a class of carcinogenic compounds that are implicated by several studies for their potential to induce oxidative stress [266-268]. Benzo(a)pyrene (BaP) is a prototype PAH, which has been widely studied for its carcinogenicity, genotoxicity, and mutagenicity [269-272]. BaP is also known to induce CYP enzymes, especially CYP1A isoforms, which can have a direct impact on the biological disposition of various drugs [253, 273]. $\mathrm{BaP}$ is metabolically activated by CYP $1 \mathrm{~A} 1 / 1 \mathrm{~B} 1$ enzymes into epoxide intermediates, which are further metabolized by CYPs or epoxide hydrolase into carcinogenic diol products $[220,274]$. As a result of CYP-mediated BaP metabolism, excessive reactive oxygen species (ROS) are generated, leading to oxidative stress [275, 276]. Oxidative stress further leads to oxidative DNA damage, lipid peroxidation, and the oxidation of several proteins, ultimately causing cytotoxicity and cell death [277, 278]. Recently, we have demonstrated that exposure of $\mathrm{BaP}$ causes the induction of CYPs and a subsequent increase in oxidative stress and cytotoxicity in U937 monocytic cells [273]. Oxidative stress is implicated in enhanced replication of HIV-1 via the activation of redox sensitive nuclear transcription factor Kappa- B (NF- $\mathrm{B})$ [192, 279-281]. Various

\footnotetext{
* Reprinted with permission. Ranjit, S., Sinha, N., Kodidela, S., Kumar, S. Benzo(a)pyrene in Cigarette smoke enhances HIV-1 replication through NF- $\kappa B$ activation via CYP-mediated oxidative stress pathway. Scientific Reports, 2018. 8:10394 | DOI:10.1038/s41598-018-28500-zpone.0163827
} 
stress factors regulate the NF- $\kappa \mathrm{B}$ pathway resulting in the transcription of over hundreds of genes that regulate inflammation, immune response, cell proliferation, growth, and survival [282-284]. NF- $\mathrm{BB}$ is activated by a number of triggers such as viral proteins and drugs of abuse, leading to the expression of various cytokines, chemokines, and CYPs [282, 285-287]. Interestingly, most of the stress factors use ROS as a secondary messenger to modulate NF- $\kappa \mathrm{B}$ activity [288]. In an inactive state, NF- $\kappa \mathrm{B}$ proteins are localized in the cytoplasm by forming a complex with inhibitors of NF- $\kappa$ B proteins (IKB) proteins. ROS triggers the activation of the IKB kinase complex that facilitates the ubiquitination of IKB proteins, thereby releasing the NF- $\kappa B$ proteins into the nucleus [289]. Within the nucleus, the activated NF- $\kappa \mathrm{B}$ proteins induce the transcription of HIV-1 structural genes by binding to the enhancer region of long terminal repeat (LTR) on HIV1 DNA, that contains NF- $\mathrm{BB}$ binding sites [290]. Several reports have emphasized the role of ROS in the activation of NF- $\kappa \mathrm{B}$ and its subsequent impact on HIV-1 gene transcription [281, 291]. However, whether smoking/tobacco mediated oxidative stress via CYP pathways causes the nuclear trafficking of NF- $\kappa B$ and resultant HIV-1 replication, is yet to be examined. In the current study, we examined the potential role of CYP-mediated oxidative stress and subsequent HIV-1 replication via the NF- $\kappa$ B pathway by an important tobacco constituent, BaP, in HIV-1-infected macrophages. We used macrophages in this study because they are a secondary target of HIV-1 infection and a major viral reservoir where it is difficult to effectively suppress the virus with antiretroviral agents [292, 293]. Moreover, HIV-1-infected macrophages cross the bloodbrain-barrier (BBB) and infect CNS cells such as perivascular macrophages, microglia, and to some extent astrocytes, which eventually cause HIV-1-associated neurocognitive disorders [294, 295].

\section{Materials and Methods}

\section{Cell Culture and Treatment}

U1 cells. U1 cells, which are U937 cells chronically infected with HIV-1, were obtained from the NIH AIDS Reagent Program (Germantown, MD). The cells were cultured in Roswell Park Memorial Institute (RPMI) 1640 media containing 10\% Fetal bovine serum (FBS) and penicillin. To differentiate the cells into macrophages, 0.8 million cells were seeded in $1.5 \mathrm{ml}$ of media containing $100 \mathrm{nM}$ phorbol 12-myristate 13acetate (PMA) in each well of a 6-well plate. After 3 days, the media containing PMA and non-adherent cells was removed and the differentiated cells were washed with phosphate buffer saline (PBS). The cells were topped with fresh $1 \mathrm{ml}$ media and treated with $\mathrm{BaP}(1 \mu \mathrm{M})$ every 24 hours for 3 days for acute treatment. An additional $0.5 \mathrm{ml}$ of media was added and $\mathrm{BaP}$ concentration was maintained constant at every treatment. In order to monitor the chronic (7 days) effect of $\mathrm{BaP}$, we initially treated U1 cells with $\mathrm{BaP}$ (10-100 $\mathrm{nM})$ for 7 days and later differentiated them into macrophages.

Human primary macrophages infected with HIV-1. Human primary macrophages were derived from the whole blood of healthy individuals using a method described in Chapter 4 . The macrophages were treated with polybrene $(2 \mu \mathrm{g} / \mathrm{ml})$ and IL-2 
(interleukin-2, $10 \mathrm{ng} / \mu \mathrm{l})$ and infected with HIV-Ada strain $(20 \mathrm{ng} / 1 \mathrm{million}$ cells $/ \mathrm{ml}$ media). The infected cells were then treated with $\mathrm{BaP}(100 \mathrm{nM})$ every 24 hours for 7 days.

\section{Viral Load}

The viral load of HIV-1 was determined by assessing the level of p24 antigen in the supernatant collected from the treated U1 cells and primary macrophages. We used the HIV-1 p24 Antigen ELISA kit (Zeptometrix Corporation, Buffalo, NY) for this purpose. The kit is comprised of monoclonal antibody- coated microwells, which specifically bind the HIV-1 p24 antigen in the added samples. The captured antigen was incubated with biotin conjugated human anti-HIV-1 antibody at $37^{\circ} \mathrm{C}$ for $1 \mathrm{hr}$, followed by incubation with enzyme, streptavidin-peroxidase and tetramethylbenzidine substrate at $37^{\circ} \mathrm{C}$ and room temperature/dark, respectively, for 30 minutes each. The reaction of the enzyme with the substrate developed a blue color, the absorbance of which was measured at $450 \mathrm{~nm}$ to determine the p24 level. The optical density of the samples was compared against the standard curve.

\section{Isolation of RNA and Protein}

RNA was isolated using RNeasy Mini kit (250) (QIAGEN, Germantown, MD), following the manufacturer's protocol. The extracted RNA was quantified using Nanodrop 2000c Spectrophotometer (Thermo Fisher Scientific) at $260 \mathrm{~nm}$. To isolate the protein from the treated cells, $100 \mu 1$ of RIPA buffer was added to the cell pellet. The cell suspension was sonicated for 30 seconds with pulse set at 4 and centrifuged at $13000 \mathrm{rpm}$ for 5 minutes. The supernatant containing the protein was collected and protein quantification was done by using the BCA protein assay kit (Thermo Fischer Scientific).

\section{Quantitative Reverse Transcriptase Polymerase Chain Reaction (RTPCR)}

The relative mRNA fold expression level of CYPs (CYP1A1 and CYP3A4) and AOEs (Catalase, SOD1) after chronic BaP treatment was monitored using quantitative RTPCR as described in Chapter 4.

\section{Western Blotting}

We determined the protein expression level of CYPs (CYP1A1 and CYP3A4) and AOEs (Catalase, SOD1) after chronic BaP treatment in U1 cells using Western blotting as described in Chapter 4. In addition to CYPs and AOEs, we also monitored the expression of NFKB subunits, p50 and p65 in the cytoplasmic and nuclear fractions of protein. We used $\beta$-lamin as a loading control for the nuclear protein fractions. We used 


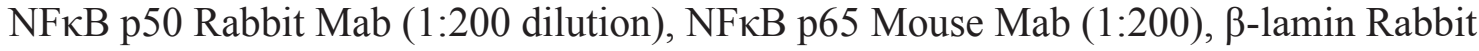
Mab (1:400 dilution) from Santa Cruz Biotechnology. Inc. Dallas, TX.

\section{Measurement of ROS Level}

We quantified ROS generated after acute (3 days) treatment of $\mathrm{BaP}$ on U1 cells using the NovoCyte flow cytometer (ACEA, Biosciences Inc., San Diego, CA). We followed the method as described in Chapter 4 for the measurement of ROS level in the cells. The data were analyzed by using the NovoExpress software.

\section{7-Ethoxy-Resorufin-O-Deethylase (EROD) Assay}

We used the EROD assay to determine the activity of CYP1A1 after the chronic treatment of BaP. Briefly, the treated cells were washed with PBS and suspended in 900 $\mu 10.1 \mathrm{M}$ Hepes buffer, $\mathrm{pH}$ 7.4. The cell suspension was then sonicated for 10 seconds, 3 times, with pulse on and off. The cell extract $(90 \mu 1)$ thus obtained was added with $1 \mu 1$ of substrate, 7-ethoxyresorufin $(100 \mu \mathrm{M})$ and incubated at $37^{\circ} \mathrm{C}$ for 10 minutes. Later, $10 \mu \mathrm{l}$ NADPH $(10 \mathrm{mM})$ was added to the reaction mixture and incubated for another 15 minutes. The reaction was stopped by using $75 \mu$ fluorescamine solution in acetonitrile $(150 \mu \mathrm{g} / \mathrm{ml})$. When the cell extracts were incubated with the substrate and NADPH, the CYP1A1 enzyme present in the cells reacted with the substrate resulting in the formation of a fluorescence product, resorufin. The fluorescence thus generated was measured at excitation/emission wavelengths of 535/590 $\mathrm{nm}$. The CYP1A1 activity was calculated by measuring the amount of fluorescence generated per unit time. The quantity of resorufin in the samples was calculated by comparing it with the standard calibration curve prepared by using 0-100 pmol of resorufin standard.

\section{Apoptotic DNA Damage}

We used the Apoptag® Iso Dual Florescence Apoptosis Detection kit (Millipore Sigma, Burlington, Massachusetts) to determine apoptotic DNA damage after BaP treatment in U1 cells. The assay was carried out using the manufacturer's protocol.

\section{Statistical Analysis}

All the data are presented as Mean \pm SEM of at least three independent experiments. The mean value obtained for the control group was normalized to $100 \%$ or 1 -fold, to which the treated cells were compared as a \% or fold of control, respectively. Student's t-test or one- or two-way ANOVA were used to calculate the statistical differences $(\mathrm{p} \leq 0.05)$ between the control and the treated groups, where applicable. All the statistical analyses were performed using GraphPad Prism 7 (San Diego, CA). 


\section{Results}

\section{BaP Induces HIV-1 Replication in U1 Cells and HIV-1-infected Human Primary Macrophages}

Chronic exposure of BaP (100 nM for 7 days) showed an approximately 4-fold increase in HIV-1 replication in U1 cells (Figure 5-1A). However, a 10-fold lower concentration of $\mathrm{BaP}(10 \mathrm{nM})$ did not have any significant effect on the viral replication. We also confirmed this result in HIV-1-infected human primary macrophages, in which, $\mathrm{BaP}(100 \mathrm{nM})$ showed an approximately 2-fold increase in HIV-1 replication

(Figure 5-1B). Furthermore, we examined apoptotic DNA fragmentation in HIV-1infected human primary macrophages after 3 days exposure of $\mathrm{BaP}(100 \mathrm{nM})$. The fluorescent images revealed an increased apoptotic DNA fragmentation with DNase Type I ends in cells treated with $\mathrm{BaP}(100 \mathrm{nM})$ compared to that of the control (Figure 5-1C). DNA fragmentation with DNase Type II ends were not visible in both the control and the treated cells. The results suggest that $\mathrm{BaP}$ induces DNA fragmentation during the early phase of apoptosis, within the nucleus of the treated cells. Upon validating the results of the U1 cells in human primary macrophages, we performed the subsequent experiments that examined the underlying mechanism in U1 cells.

\section{BaP Induces the Expression of CYP1A1}

The expression of CYP1A1 and CYP3A4, which are the major BaP-metabolizing CYPs, were examined in U1 cells exposed to $\mathrm{BaP}(100 \mathrm{nM})$ for 7 days. The chronic exposure of BaP showed an approximately 30-fold increase in the mRNA expression of CYP1A1 in U1 cells (Figure 5-2A), but failed to show any significant expression of CYP1A1 at the protein level (Figure 5-2B). The chronic exposure of BaP (100 nM) increased the enzymatic activity of CYP1A1 by approximately 2-fold (Figure 5-2C). On the other hand, we did not observe any significant change in the expression of CYP3A4 at both the mRNA and protein levels (Figure 5-2D-E).

\section{BaP Does Not Alter the Expression of AOEs}

The induction of CYPs could metabolize $\mathrm{BaP}$ and increase ROS. However, the ROS could subsequently be neutralized by the induction of antioxidant enzymes (AOEs). Therefore, we examined the induction of two of the most important and general AOEs; superoxide dismutase 1 (SOD1) and catalase by $100 \mathrm{nM} \mathrm{BaP}$. Our results demonstrated no significant change in the mRNA and protein expression levels of both SOD1 and catalase (Figure 5-3A-D). 


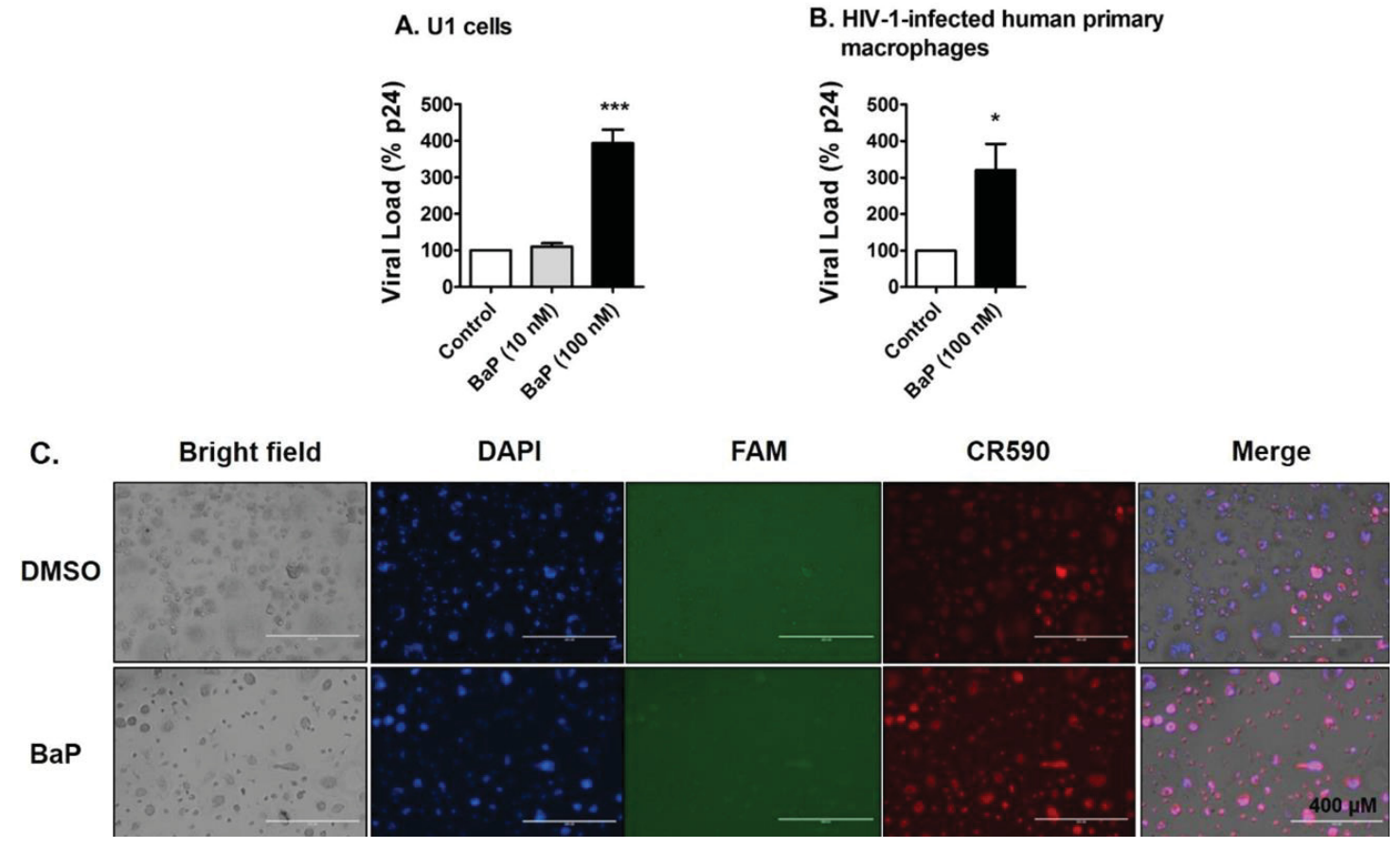

B. HIV-1-infected human primary macrophages

Figure 5-1. Chronic treatment of BaP induces HIV-1 replication and apoptotic DNA damage in HIV-1-infected macrophages.

(A). The U1 cells were treated with $10 \mathrm{nM}$ and $100 \mathrm{nM} \mathrm{BaP}$ for seven days. After the $\mathrm{BaP}$ treatment, the $\mathrm{U} 1$ cells were stimulated with $100 \mathrm{nM}$ of Phorbol 12-myristate 13acetate (PMA) to produce HIV-1. Supernatants were collected after two days of differentiation, which were used for the p24 ELISA assay to assess the viral load. The chronic (7 days) treatment of $\mathrm{BaP}(100 \mathrm{nM})$ significantly increased the viral replication in U1 cells, while the 10 -fold lower concentration did not have any significant effect. The data is displayed as mean $\pm \operatorname{SEM}(\mathrm{n}=6)$, calculated as a percentage of the control. (B). HIV-1-infected human primary macrophages were treated with $\mathrm{BaP}(100 \mathrm{nM})$ for 3 days. The supernatant was collected thereafter and used for the p24 ELISA assay to assess the viral load. The acute (3 days) treatment of $\mathrm{BaP}(100 \mathrm{nM})$ significantly increased the viral replication in HIV-1-infected macrophages. The data is displayed as mean $\pm \operatorname{SEM}(n=4)$, calculated as percentage of the control. The statistical significance was calculated at $\mathrm{p} \leq$ $0.05(*)$, where $* * *$ represents $\mathrm{p} \leq 0.0005$, compared with the control group. $(\mathbf{C})$. The apoptotic DNA damage assay was performed on the treated cells. DAPI, FAM and CR590 stained nucleus (blue), apoptotic DNA damage with DNase Type II ends (green) and Type I ends (red) respectively. A higher signal for CR590 is visible in the fluorescent images, indicating apoptotic DNA fragmentation with DNase Type I ends in the infected human primary macrophages after $\mathrm{BaP}(100 \mathrm{nM})$ exposure for 3 days. DNA fragmentation with DNase Type II ends (green) was not visible in either the control or the treated cells. Therefore, the images indicate that $\mathrm{BaP}(100 \mathrm{nM})$ induces DNA fragmentation during the early phase of apoptosis in the HIV-1-infected human primary macrophage 
A.

CYP1A1

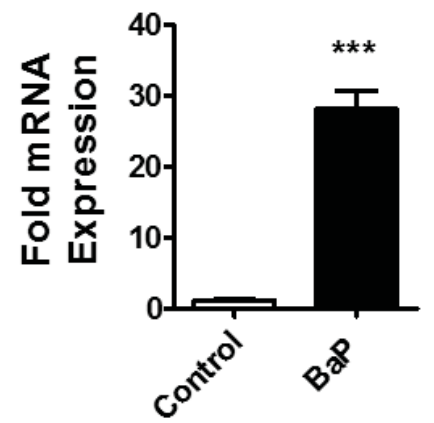

B.
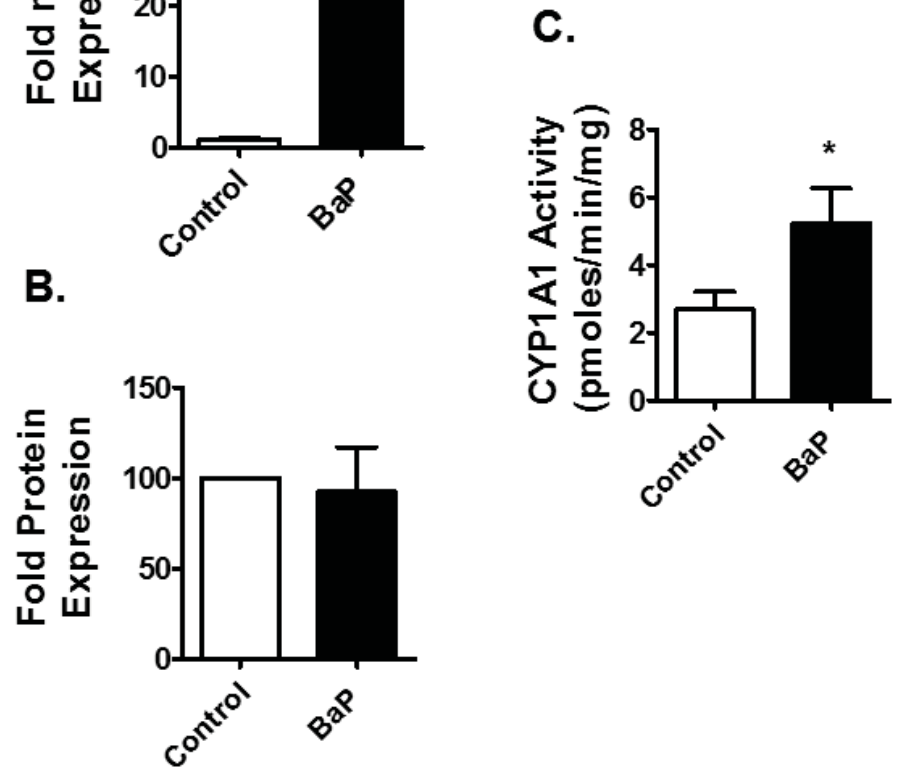

D.

CYP3A4

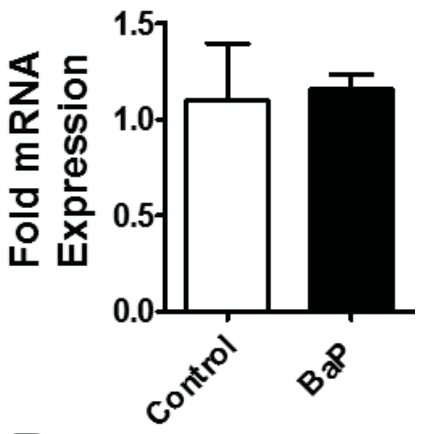

E.
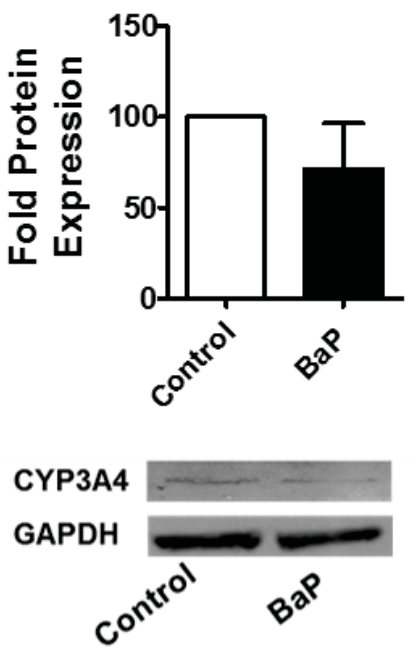

Figure 5-2. Chronic treatment of BaP induces expression of CYPs in U1 cells. The U1 cells were treated with $100 \mathrm{nM} \mathrm{BaP}$ for seven days. We measured the mRNA expression $(\mathbf{A}, \mathbf{D})$ and protein expression $(\mathbf{B}, \mathbf{E})$ of CYPs $(1 \mathrm{~A} 1,3 \mathrm{~A} 4)$ using RTPCR and western blotting, respectively. Chronic exposure of $\mathrm{BaP}(100 \mathrm{nM})$ significantly induced the expression of CYP1A1 at the mRNA level, but not at the protein level. Therefore, we measured activity of CYP1A1 using the EROD assay $(\mathbf{C})$. Chronic BaP treatment increased CYP1A1 activity by approximately 2 -fold. However, there was no significant change in the expression of CYP3A4 at both the mRNA and protein levels. The data are displayed as mean \pm SEM of at least three independent experiments $(n \geq 3)$. The mRNA/protein expression of the treated cells are normalized to control cells, whose expression was set at 1-fold. GAPDH was used as an endogenous control and loading control for RTPCR and western blotting, respectively. The statistical significance was calculated at $\mathrm{p} \leq 0.05(*)$ compared with the control group. The blots are representative of at least three independent experiments. 
A.

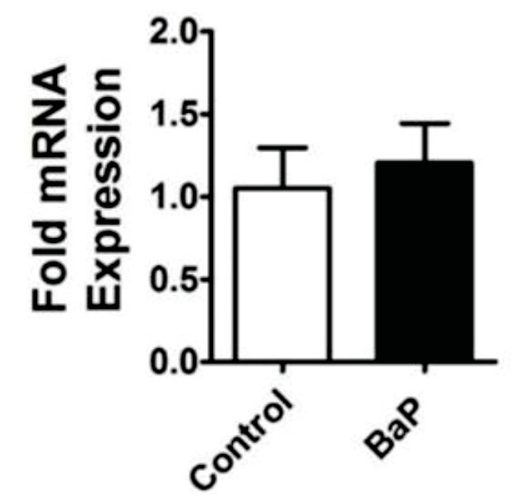

B.

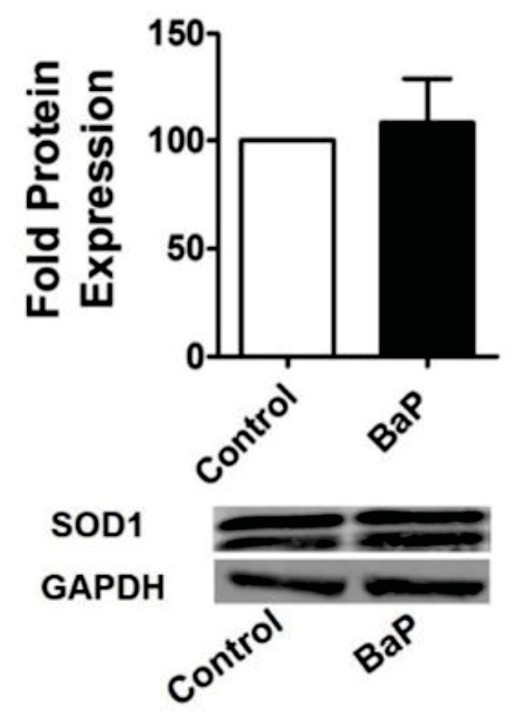

D. Catalase

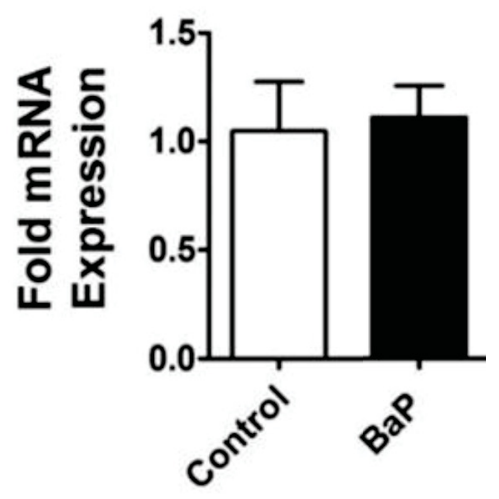

C.

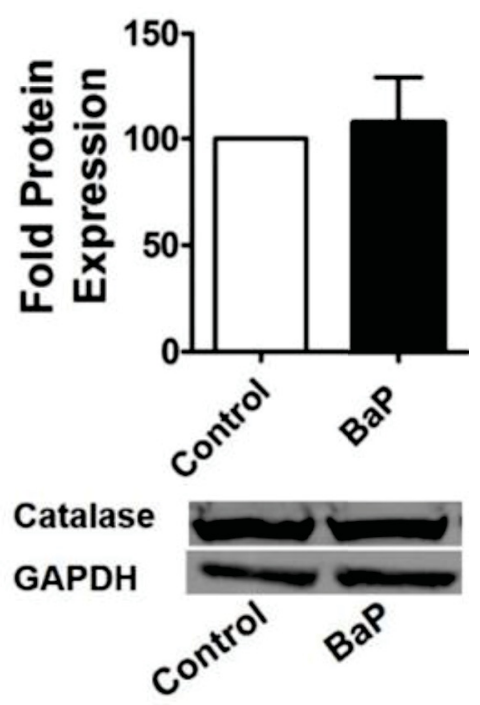

Figure 5-3. Chronic treatments of BaP have no significant effect on the expression of AOEs in $\mathrm{U} 1$ cells.

The U1 cells were treated with $100 \mathrm{nM} \mathrm{BaP}$ for seven days. We measured the mRNA expression (A, D) and protein expression (B, E) of AOEs (SOD1 and Catalase) using RTPCR and western blotting, respectively. There was no significant change in the expression of SOD1 and catalase at both the mRNA and protein levels after chronic treatment of $\mathrm{BaP}(100 \mathrm{nM})$. The data are displayed as mean $\pm \mathrm{SEM}$ of at least three independent experiments $(n \geq 3)$. The mRNA/protein expression of treated cells are normalized to control cells, whose expression was set at 1-fold. GAPDH was used as an endogenous control and loading control for RTPCR and western blotting, respectively. The statistical significance was calculated at $\mathrm{p} \leq 0.05(*)$ compared with the control group. The blots are representative of at least three independent experiments. 


\section{Role of CYP1A1 on BaP-induced ROS Generation}

Treatment of $\mathrm{BaP}(1 \mu \mathrm{M})$ in $\mathrm{U} 1$ cells for 3 days increased the generation of ROS by $30 \%$ (Figure 5-4A-B). In this and the subsequent experiments we used $1 \mu \mathrm{M}$ BaP for 3 days to induce oxidative stress and/or HIV-1 replication, because chemical inhibitors and antioxidants are toxic to the cells when treated for 7 days. Further, we pre-treated the $\mathrm{U} 1$ cells with different antioxidants or $\mathrm{CYP}$ inhibitors prior to $\mathrm{BaP}$ exposure to determine whether these compounds reduce the BaP-induced ROS generation. We used vitamin $\mathrm{C}$ $(100 \mu \mathrm{M})$, vitamin $\mathrm{E}(100 \mu \mathrm{M})$, resveratrol $(50 \mu \mathrm{M})$, a resveratrol analog, pinostilbene $(2$ $\mu \mathrm{M})$ as antioxidants, and a selective CYP1A1 inhibitor, ellipticine $(1 \mu \mathrm{M})$ for the study at the concentrations previously shown to be effective [296, 297]. Vitamin C and ellipticine significantly reduced the BaP-induced production of ROS (Figure 5-4A-B). However, there was no significant change in $\mathrm{BaP}$-induced $\mathrm{ROS}$ after treatment with vitamin $\mathrm{E}$, resveratrol, and pinostilbene. Figure 5-4C shows the graphical representation of the results obtained in Figure 5-4A-B.

\section{Role of CYP1A1 and Oxidative Stress Pathways on BaP-induced HIV-1 Replication}

The $\mathrm{U} 1$ cells treated with $\mathrm{BaP}(1 \mu \mathrm{M})$ for 3 days showed approximately $70 \%$ increase in HIV-1 replication (Figure 5-5A). Treatment with antioxidants, vitamin $\mathrm{C}$ and $\mathrm{E}(100 \mu \mathrm{M}$ each $)$ and, resveratrol $(50 \mu \mathrm{M})$ rescued the viral replication caused by BaP (1 $\mu \mathrm{M})$ (Figure 5-5A). In addition, treatment with the CYP1A1 inhibitor, ellipticine $(1 \mu \mathrm{M})$ also exhibited a reduction in viral load in BaP-treated U1 cells (Figure 5-5B). To further validate our results, we knocked down the CYP1A1 gene in the U1 cells, using a siRNA silencing technique prior to $\mathrm{BaP}$ treatment. The viral load significantly decreased after silencing the CYP1A1 gene (Figure 5-5C), which further confirms a role of CYP1A1 on BaP-induced HIV-1 replication.

\section{Nuclear Translocation of NF-кB Subunits upon BaP Exposure}

There was no prominent change in the expression level of NF- $\kappa \mathrm{B}$ p50 and p65 in the cytoplasmic fraction after chronic (Figure 5-6A, left panel) and acute (Figure 5-6B, left panel) treatment of $\mathrm{BaP}(100 \mathrm{nM})$ and $\mathrm{BaP}(1 \mu \mathrm{M})$, respectively. Interestingly, there was an increase in the expression of both the NF- $\kappa$ B proteins, especially $p 65$, in the nuclear fraction after acute and chronic $\mathrm{BaP}$ exposures compared to the control (Figure 5-6A-B, right panel).

\section{Role of NF-кB Pathway in BaP-induced HIV-1 Replication}

Treatment of BaP $(1 \mu \mathrm{M})$-exposed U1 cells with NF- $\kappa \mathrm{B}$ inhibitors such as IKK16 (Figure 5-7A) or SC-514 (Figure 5-7B) for 3 days, significantly reduced HIV-1 replication in $\mathrm{BaP}$-exposed $\mathrm{U} 1$ cells, suggesting that the viral replication occurred via the $\mathrm{NF}-\kappa \mathrm{B}$ signaling pathway. We also monitored the translocation of $\mathrm{NF}-\kappa \mathrm{B}$ p 65 , the major 
A.

B.
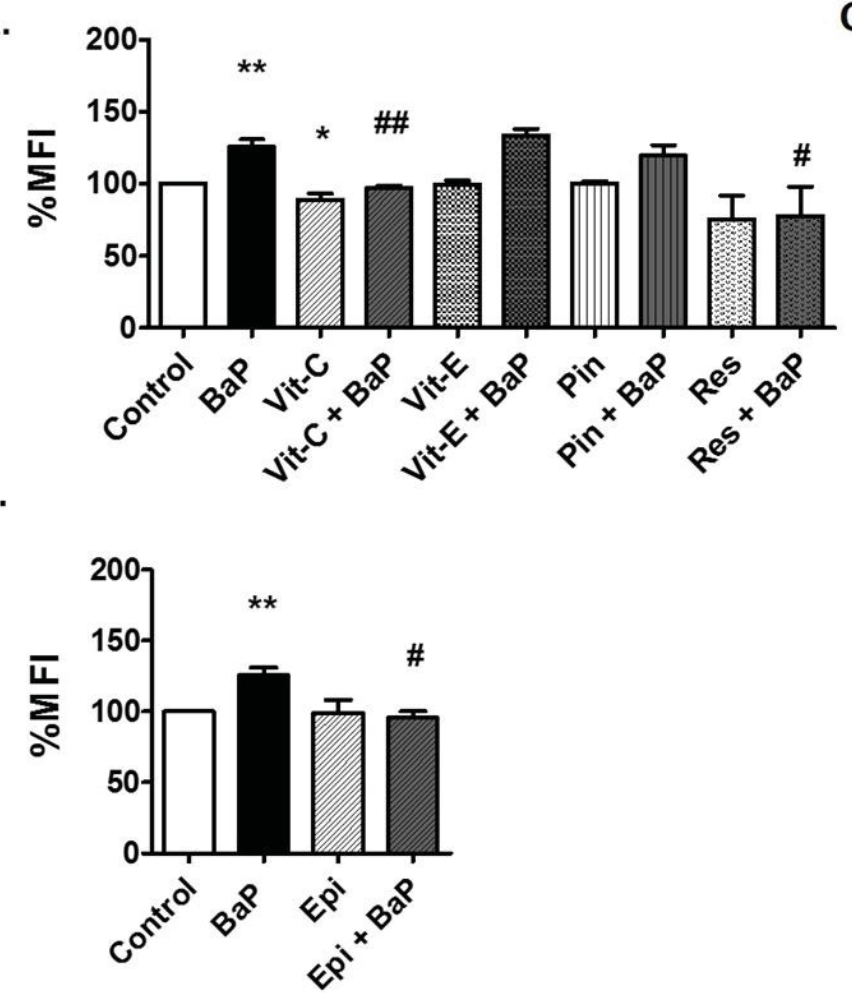

C.
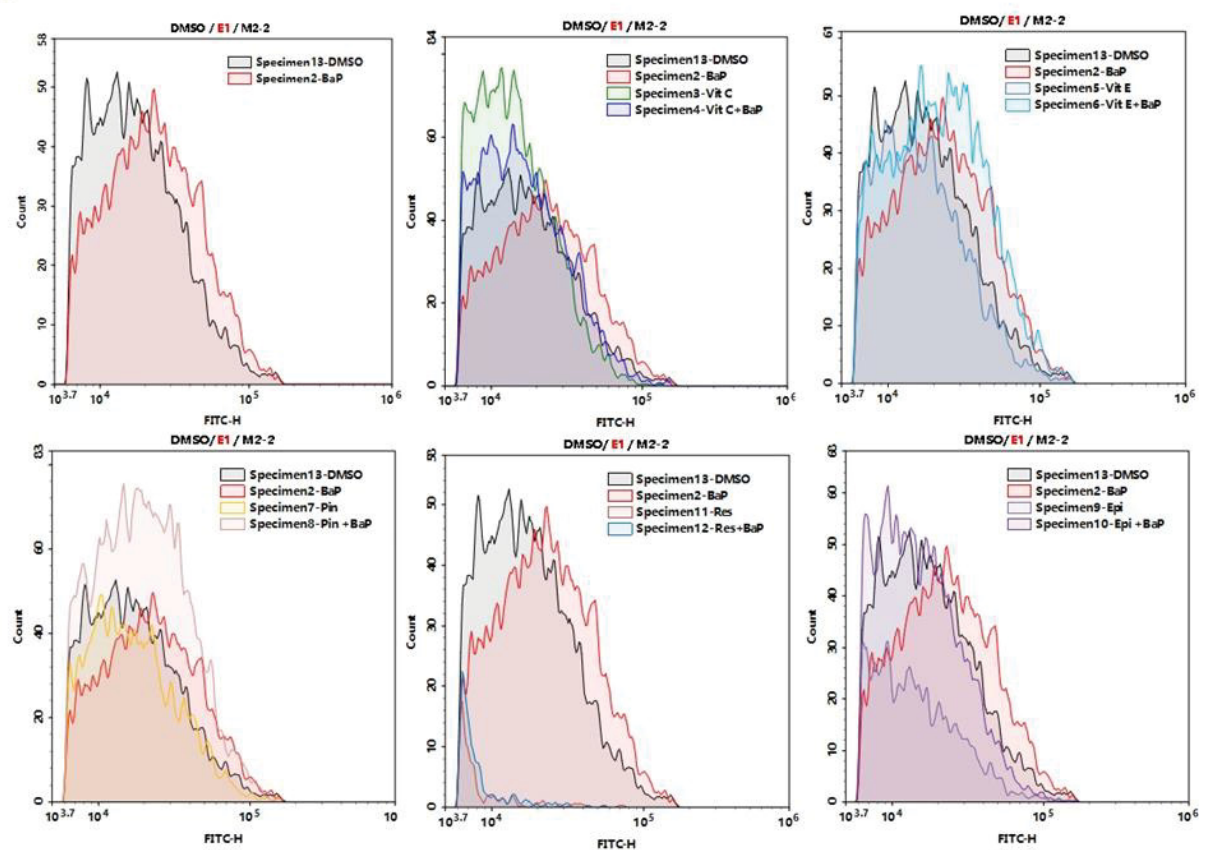

Figure 5-4. Treatment of antioxidants and CYP1A1 inhibitors reduce ROS in U1 cells due to BaP exposure.

U1 cells were concomitantly treated with $\mathrm{BaP}(1 \mu \mathrm{M})$ and antioxidants [vitamin $\mathrm{C}(100 \mu \mathrm{M})$, vitamin $\mathrm{E}(100 \mu \mathrm{M})$, pinostilbene $(2 \mu \mathrm{M})$, and resveratrol $(50 \mu \mathrm{M})$ ] $(\mathbf{A})$ or a CYP1A1 inhibitor [ellipticine $(1 \mu \mathrm{M})$ ] $(\mathbf{B})$. Treated cells were stained with CMDCFDA dye and the fluorescence emitted was measured using flow cytometry at excitation/ emission of 495/519 nm.

Treatment of $\mathrm{BaP}(1 \mu \mathrm{M})$ significantly increased ROS in $\mathrm{U} 1$ cells, which was rescued by the treatment of vitamin $\mathrm{C}$, and ellipticine. The data were obtained from the mean of at least three independent experiments. $*$ and $* *$ Represents $\mathrm{p} \leq 0.05$ and $p \leq 0.005$ respectively, compared with the control group while \# and \#\# represent $p \leq 0.05$ and $p \leq 0.005$, respectively, compared to the BaP-treated groups. Figure $\mathbf{C}$. shows the graphical representation of mean fluorescence intensity due to the treatmen 

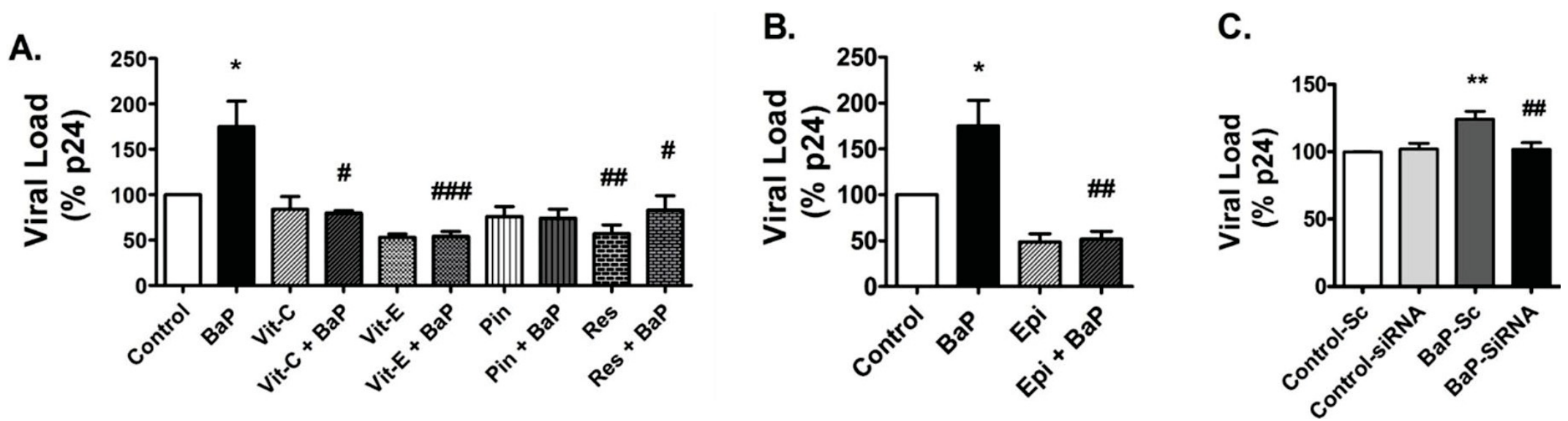

Figure 5-5. Treatment of antioxidants and CYP1A1 inhibitors reduce HIV-1 replication in U1 cells due to BaP exposure.

U1 cells were concomitantly treated with $\mathrm{BaP}(1 \mu \mathrm{M})$ and antioxidants [vitamin $\mathrm{C}(100 \mu \mathrm{M})$ and vitamin $\mathrm{E}(100 \mu \mathrm{M})$, pinostilbene $(2 \mu \mathrm{M})$, and resveratrol $(50 \mu \mathrm{M})](\mathbf{A})$ or CYP1A1 inhibitor epiliptine $(1 \mu \mathrm{M})]$ (B) for 3 days. Prior to BaP treatment, the CYP1A1 gene was knocked down in the U1 cells using siRNA specific to CYP1A1 $(\mathbf{C})$. The cells were then treated with $\mathrm{BaP}(100 \mathrm{nM})$ for 3 days. After the treatment, supernatants were collected to determine the viral load using the p24 ELISA assay. HIV-1 replication significantly increased with 3-days exposure of $\mathrm{BaP}(1 \mu \mathrm{M})$, which was rescued by all the antioxidants (vitamin $\mathrm{C}$ and $\mathrm{E}$, and resveratrol) as well as the CYP1A1 inhibitor, epilliptine. The knock-down of the CYP1A1 gene also rescued HIV-1 replication in BaP-exposed U1 cells. The data were obtained from the mean of at least three independent experiments. ${ }^{*}$ and $* *$ Represents $\mathrm{p} \leq 0.05$ and $\mathrm{p} \leq 0.005$ compared with the control group while \#, \#\# and \#\#\# represents $\mathrm{p} \leq 0.05, \mathrm{p} \leq 0.005$ and $\mathrm{p} \leq 0.0005$, respectively, compared to the BaP-treated groups. 
A.

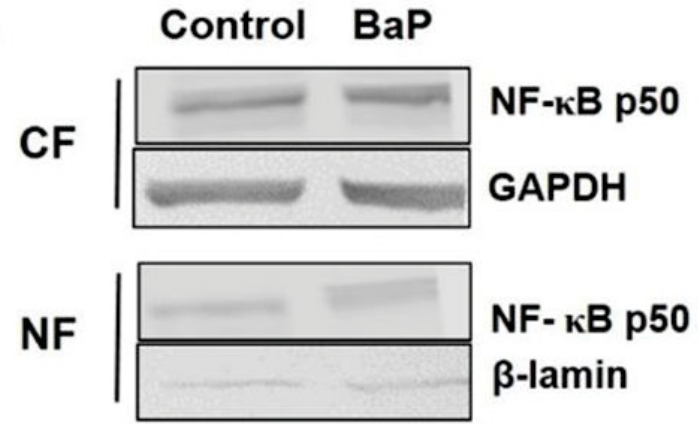

B.

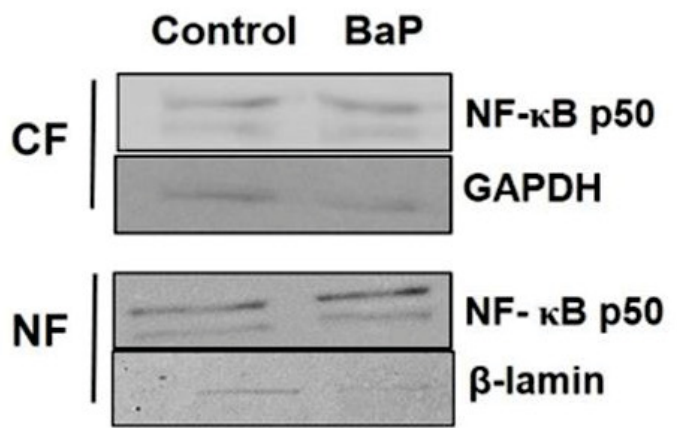

Control BaP

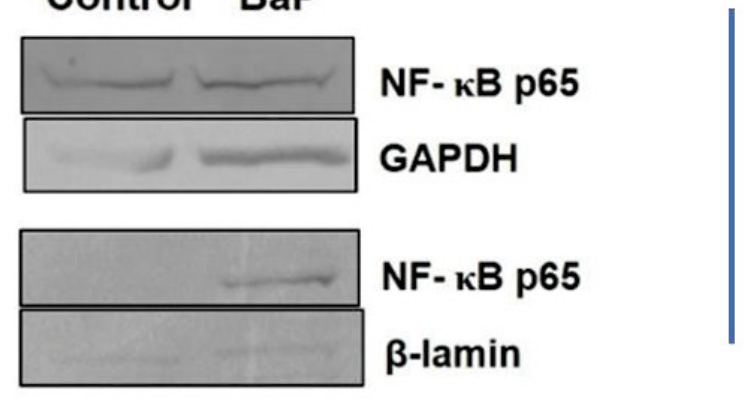

Chronic exposure

Control BaP

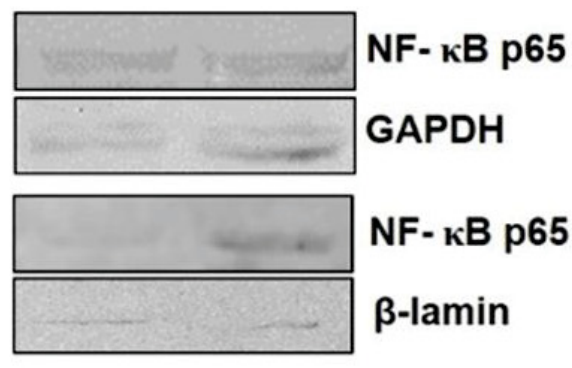

\section{Acute exposure}

Figure 5-6. Translocation of NF-кB subunits from cytoplasm to nucleus upon BaP exposure.

U1 cells were treated with $\mathrm{BaP} 100 \mathrm{~nm}(\mathbf{A})$ and $1 \mu \mathrm{M}$ (B) for 7 days and 3 days, respectively. After the treatment, proteins from the cytoplasm and nucleus were extracted from the cells. Western blot was run to determine the expression of the NF- $\mathrm{B}$ p50 and p65 subunits in the proteins in cytosolic fraction (CF) and nuclear fraction (NF). GAPDH and $\beta$-lamin were used as loading controls for the cytoplasmic and nuclear proteins, respectively. The blots are representative of at least three independent experiments. There is not much difference in the expression of NF- $\kappa$ B p50 and p 65 between the control and the BaP-treated cells. However, there is a clear increase in the expression of both the subunits in the nuclear fraction of acutely or chronically BaP-treated cells compared to the control group. 
A.

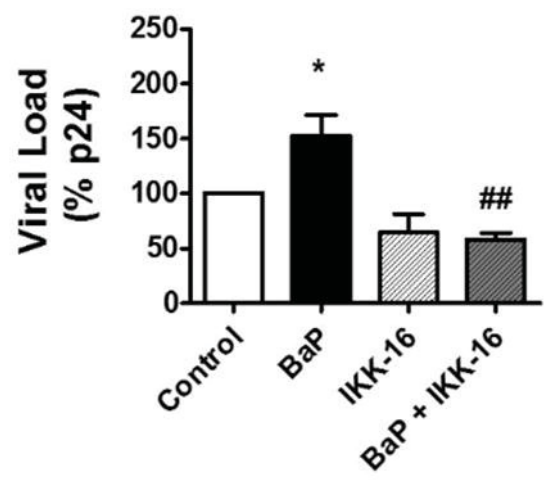

B.

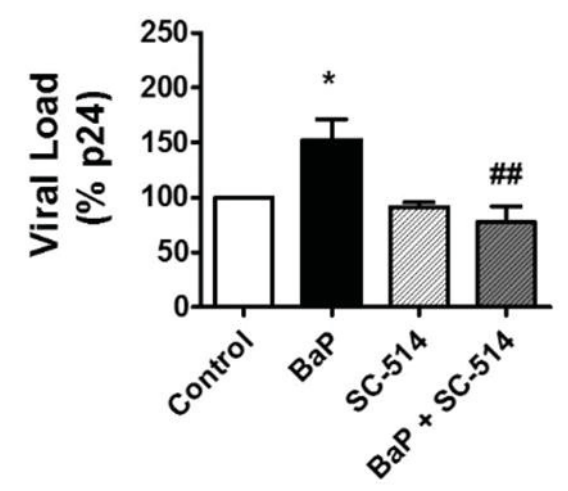

c.

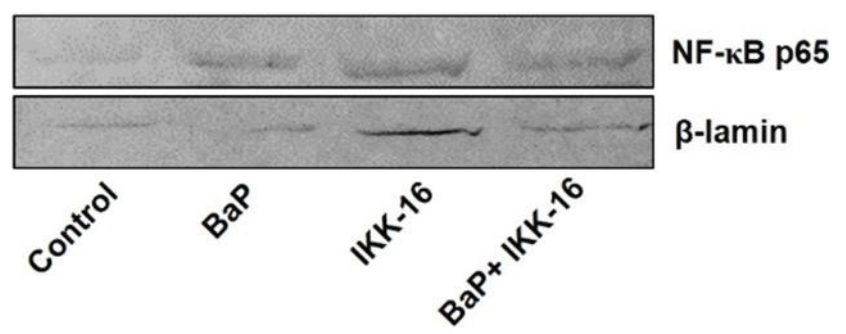

D.

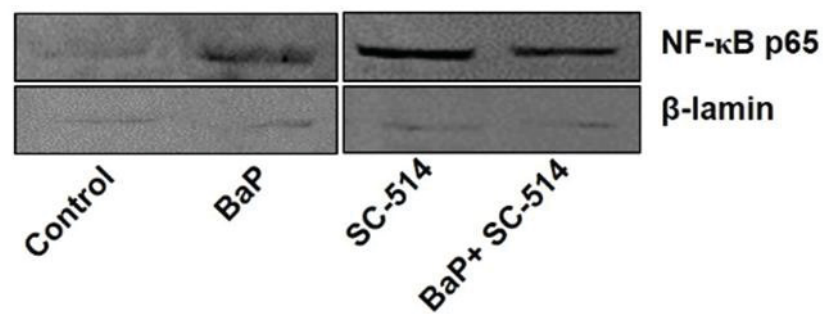

E.

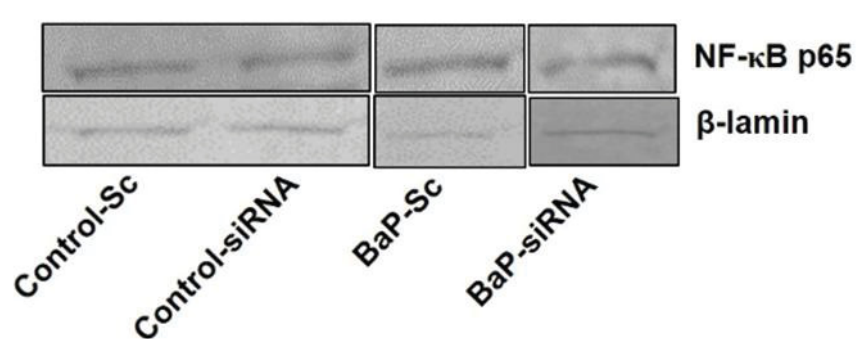

Figure 5-7. Treatment of NFK-B inhibitors reduce HIV-1 replication in U1 cells due to BaP exposure.

U1 cells were concomitantly treated with $\mathrm{BaP}(1 \mu \mathrm{M})$ and NFK-B inhibitors, IKK-16 (400 nM) (A), and SC-515 (10 $\mu \mathrm{M})(\mathbf{B})$ for 3 days. After the treatment, supernatants were collected to determine the viral load using the p24 ELISA assay. HIV-1 replication due to $\mathrm{BaP}(1 \mu \mathrm{M})$ exposure was significantly rescued by NFk-B inhibitors, IKK-16 (400 nM) and SC-514 (10 $\mu \mathrm{M}) . *$ Represents $\mathrm{p} \leq 0.05$ compared with the control group while \#\# represents $\mathrm{p} \leq 0.005$ compared to the BaP-treated groups. Western blots were run using the nuclear fraction proteins obtained from the BaP-exposed cells treated with IKK-16 (C), SC-524 (D) or siRNA CYP1A1 (E) to determine the expression of NFא-B p65 subunits. The blots indicate that treatment

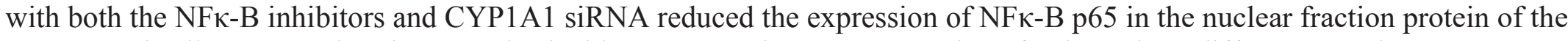
$\mathrm{BaP}$-treated cells compared to the control. The blots presented are representative of at least three different experiments. 
DNA-binding subunit of the NF- $\kappa \mathrm{B}$ protein into the nucleus, after treatment with NF- $\kappa \mathrm{B}$ inhibitors, IKK-16 (Figure 5-7C) and SC-514 (Figure 5-7D) and after siRNA silencing of CYP1A1 (Figure 5-7E). The results showed that the knock-down of CYP1A1 in BaPtreated cells reduces the translocation of the NF- $\kappa$ B p 65 unit into the nucleus. As expected, the expression of the NF- $\mathrm{NB}$ p 65 protein was also observed to be lower in the nucleus of BaP-treated cells, after treatment with IKK-16 and SC-514

\section{Discussion}

Several reports suggest that cigarette smoke exposure is associated with increased HIV-1 replication and infectivity $[140,157,211]$. However, the mechanism of smoking induced HIV-1 replication is poorly understood, except for the fact that oxidative stress is a possible mechanism for enhanced viral load $[153,154]$. The current report reveals a novel mechanism for BaP-mediated HIV-1 replication in monocyte-derived macrophages in vitro. In this study, we have demonstrated that oxidative stress generated by the CYP1A1-mediated metabolism of BaP, triggers the redox-sensitive transcription factor, NF- $\kappa \mathrm{B}$ that leads to the amplification of HIV-1. This is the first evidence of the involvement of a novel CYP-mediated oxidative stress pathway in tobacco-mediated HIV-1 replication via NF- $\kappa \mathrm{B}$ in macrophages (Figure 5-8)

Recently, we have shown that the exposure of CSC increases HIV-1 replication, oxidative stress, and induction of CYP enzymes in U937 and/or U1 macrophages [154]. We have also shown the involvement of CYP2A6 in nicotine metabolism and oxidative stress in U937 cells [230]. Further, our study using ex vivo samples from HIV-1-infected individuals who smoke also demonstrated an increase in HIV-1 replication, oxidation stress, and nicotine metabolism [153]. Taken together, these studies suggest an association of CYP-mediated oxidative stress with HIV-1 replication in tobacco smokers. Comparison of oxidative stress levels after nicotine and CSC exposure revealed that the induction of ROS by CSC is much higher than ROS induction by nicotine [230]. Therefore, we recently studied the effect of $\mathrm{BaP}$, an important $\mathrm{PAH}$ component of CSC, in U937 cells and observed that BaP increases CYP1A1 expression, ROS levels, and cytotoxicity [273]. The excessive ROS production by BaP likely disturbed the redox homeostasis, causing oxidative stress, which resulted in cytotoxicity in U937 cells [298].

In the present study, we examined whether BaP induces HIV-1 replication in HIV-1-infected macrophages via the CYP-induced oxidative stress pathway. To demonstrate this, first we studied the effect of $\mathrm{BaP}$ in HIV-1 replication in U1 cells and our findings suggested that chronic exposure of BaP (100 nM) increases HIV-1 replication in these cells. We have also confirmed this result in HIV-1-infected human primary macrophages. Furthermore, BaP exposure induced DNA fragmentation during the early phase of apoptosis. Secondly, the expression of CYP1A1 and CYP3A4, which are the major BaP-metabolizing CYPs, were examined in U1 cells after BaP (100 nM) exposure for 7 days. The results showed an increase in the mRNA expression of CYP1A1 by $\mathrm{BaP}$ which is consistent with the previous study findings [220, 273, 299, 300]. BaP induces the expression of CYPs in cells by activating a nuclear receptor, aryl 


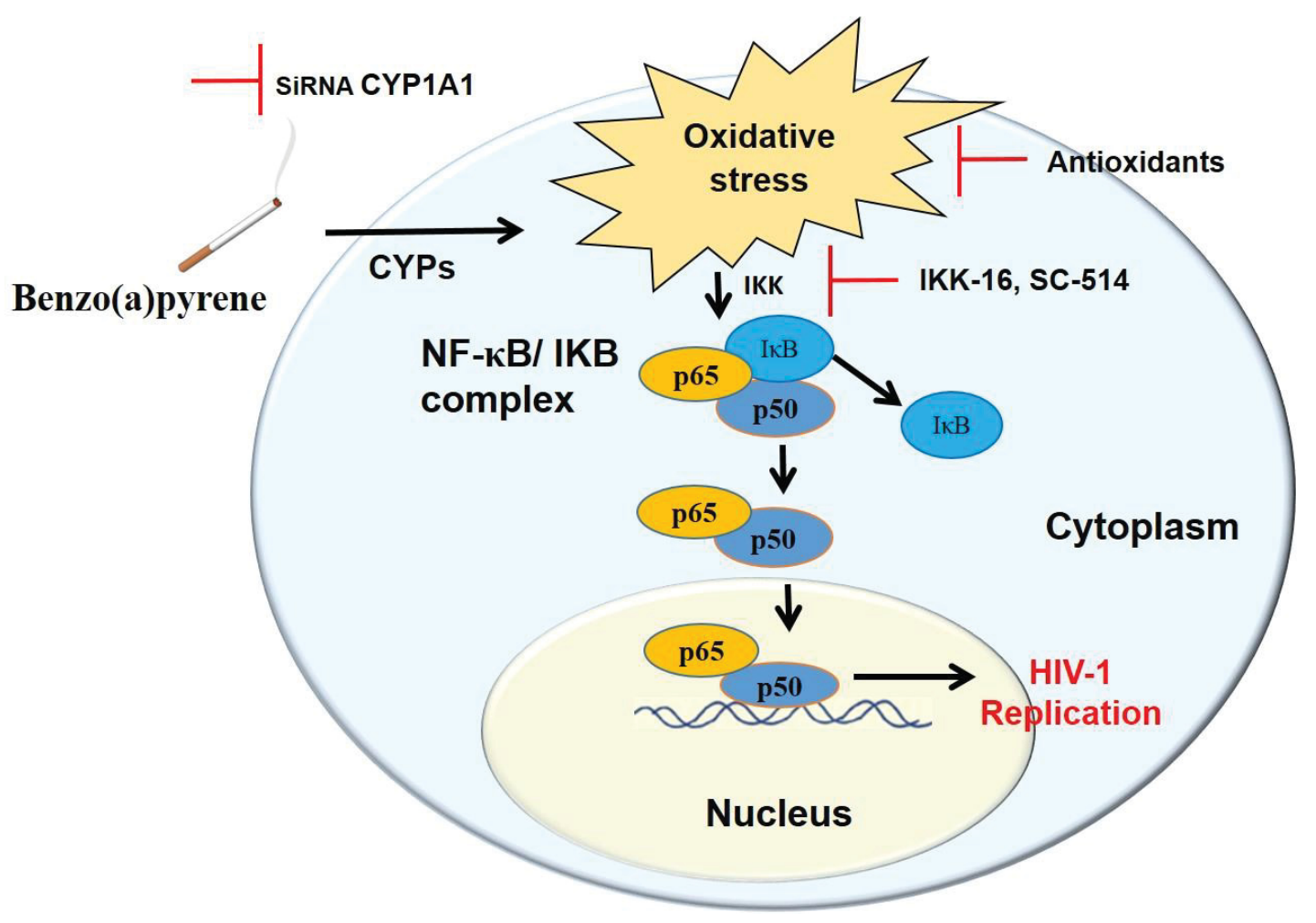

Figure 5-8. Schematic diagram showing the mechanism of BaP-induced -1-1 replication in macrophages.

Cigarette smoke constituents such as benzo(a)pyrene (BaP) induces expression of CYP1A1 in HIV-1-infected cells. CYP1A1 in turn metabolizes BaP into various BaPmetabolites, during which large amounts of reactive oxygen species (ROS) are generated. The resulting oxidative stress induces HIV-1 replication in the cells via a NF- $\mathrm{BB}$ pathway. In an inactive state, NF- $\kappa \mathrm{B}$ (p65 and p50 subunits) forms a complex with an inhibitor of NF- $\kappa$ B proteins (I $\kappa \mathrm{B})$, which inhibits its translocation from the cytoplasm to the nucleus. Oxidative stress due to BaP triggers the activation of IкB kinase (IKK) that facilitates the ubiquitination of $\mathrm{I} \kappa \mathrm{B}$ proteins, thereby releasing the NF- $\kappa \mathrm{B}$ subunits into the nucleus. Within the nucleus, the NF- $\mathrm{BB}$ proteins bind to specific DNA regions and trigger the expression of HIV-1 structural genes. Knock-down of CYP1A1 by siRNA specific to CYP1A1 and inhibition of the NF- $\kappa$ B pathway using specific NF- $\kappa B$ inhibitors such as IKK-16 and SC-514 significantly reduced HIV-1 replication in HIV-1infected macrophages. 
hydrocarbon receptor (AHR) [251]. The binding of BaP dissociates AHR from the AHRheat shock protein 90 complex, which facilitates AHR translocation into the nucleus. Inside the nucleus, AHR binds to DNA with the help of AHR nuclear translocator (ARNT) and activates the transcription of CYP1A1, CYP1A2 and CYP1B1 [301, 302]. This is a well-known pathway and therefore a similar mechanism likely occurs in inducing CYP1A1 by BaP in macrophages presented in this study. However, as seen before, we did not observe any significant expression of CYP1A1 at the protein level, perhaps due to post translational modifications of the protein or instability of the protein after extraction. Therefore, we determined its enzymatic activity using the Erod assay [303]. The chronic exposure of $\mathrm{BaP}(100 \mathrm{nM})$ increased the enzymatic activity of CYP1A1 by approximately 2 -fold. On the other hand, we did not observe any significant change in the expression of CYP3A4 at both the mRNA and protein levels. However, $\mathrm{BaP}$ induced the expression of CYP3A4 in human primary monocytes [273]. This discrepancy could be due to the use of two different, uninfected human primary monocytes and HIV-1-infected (U1), monocytic cell lines.

Thirdly, it is well known that CYPs generate ROS while metabolizing a wide range of substrates via coupling and auto oxidation reactions [304], and our results are in agreement with previous reports [276, 305], showing that acute treatment of $\mathrm{BaP}(1 \mu \mathrm{M})$ in U1 cells for 3 days increase the ROS levels. Further, we treated the U1 cells with different antioxidants or CYP inhibitors prior to $\mathrm{BaP}$ exposure to determine whether these compounds reduced the BaP-induced ROS generation. We chose a shorter duration of time and a higher $\mathrm{BaP}$ concentration to induce ROS in this experiment, because chronic treatment of antioxidants or CYP inhibitors along with $\mathrm{BaP}$ causes cytotoxicity. We used vitamin $\mathrm{C}$, vitamin $\mathrm{E}$, resveratrol, a resveratrol analog, pinostilbene as antioxidants, and a selective CYP1A1 inhibitor ellipticine to see if these antioxidants can block the BaPinduced oxidative stress. In addition to their antioxidant effects, resveratrol and pinostilbene are also known to inhibit CYP1A1 [297]. Vitamin C and ellipticine significantly reduced the $\mathrm{BaP}$-induced production of ROS. The results suggest that $\mathrm{BaP}$ induced ROS occurs via CYP1A1-mediated metabolic activation of BaP.

Cells express AOEs to neutralize the excessive accumulation of ROS generated by various stress factors $[278,306]$. $\mathrm{BaP}$ is known to induce the expression of AOEs by the activation of the nuclear Nrf2 (nuclear factor erythroid 2-related factor) pathway [307]. BaP downregulates the activity of the Nrf2 inhibitory protein, kelch-like ECHassociated protein 1(Keap1), which prevents the proteasomal degradation of Nrf2 and promotes its translocation to the nucleus, where it binds to the enhancer ARE in DNA, leading to the transcription of the AOEs [307, 308]. Therefore, we examined the induction of two of the most important and general AOEs; superoxide dismutase 1 (SOD1) and catalase in $\mathrm{U} 1$ cells after $\mathrm{BaP}$ exposure. There was no significant change in the mRNA and protein expression levels of both SOD1 and catalase. Although the basal AOEs may have played their part in scavenging ROS, the persistent increase in oxidative stress after BaP exposure suggests that their antioxidant capacity was not sufficient. Furthermore, these results suggest that the inability of $\mathrm{BaP}$ to enhance AOE expression could cause an increased generation of CYP-induced ROS by BaP. 
Next, we checked whether oxidative stress induced through the CYP1A1mediated metabolism of BaP increased HIV-1 replication in HIV-1-infected macrophages. The treatment of $\mathrm{U} 1$ cells with $\mathrm{BaP}$ showed an approximately $70 \%$ increase in HIV-1 replication, which was significantly reduced by the treatment of antioxidants such as vitamin $\mathrm{C}$ and $\mathrm{E}$, and resveratrol. In addition, treatment with the CYP1A1 inhibitor, ellipticine, also exhibited a reduction in the viral load of BaP-treated $\mathrm{U} 1$ cells. Taken together, these results suggest that BaP induces HIV-1 replication in U1 cells via the CYP-mediated oxidative stress pathway. We knocked down the CYP1A1 gene in the U1 cells, using the siRNA silencing technique prior to BaP treatment to further confirm our results. The reduction in HIV-1 replication after siRNA silencing of the CYP1A1 gene, further strengthens the role of CYP1A1 in BaP-induced HIV-1 replication.

Upon demonstrating that $\mathrm{BaP}$ enhances $\mathrm{HIV}-1$ replication in $\mathrm{U} 1$ cells via the CYP-mediated oxidative stress pathway, our next goal was to identify the molecular mechanism of oxidative stress-induced viral replication upon $\mathrm{BaP}$ exposure. Several studies have suggested that the NF- $\kappa$ B pathway triggered by ROS has a role in HIV-1 replication $[192,280]$. Therefore, we monitored the translocation of NF- $\kappa B$ subunits from the cytoplasm into the nucleus after chronic treatment of BaP in U1 cells. Since the differentiated macrophages specifically express the transcriptionally active NF- $\kappa \mathrm{B}$ p65p50 heterodimer [309], and this heterodimer is specifically expressed during NF- $\kappa \mathrm{B}-$ mediated HIV-1 transcription [310], we monitored the protein expression of these NF- $\mathrm{B}$ subunits in both the cytoplasm and the nucleus. We observed that $\mathrm{BaP}$ increases the expression of both of the NF- $\mathrm{BB}$ proteins, especially p65, in the nuclear fraction after acute and chronic $\mathrm{BaP}$ exposure. Based on these findings, we suggest that ROS generated through CYP1A1-mediated metabolism of $\mathrm{BaP}$ could trigger the NF- $\kappa \mathrm{B}$ pathway, which eventually perpetuates viral transcription. Furthermore, we confirmed the involvement of the NF- $\kappa \mathrm{B}$ pathway in $\mathrm{BaP}$-induced HIV-1 replication by treating BaP-exposed U1 cells with NF- $\kappa$ B inhibitors such as IKK-16 and SC-514. Both of the compounds inhibit NF$\kappa \mathrm{B}$ activity by selectively acting on IKK- $\beta$, an isoform of IKK protein $[311,312]$. In addition, SC-514 decreases the import of p65 into the nucleus and expedites the export of p65 from the nucleus, as well as inhibiting the phosphorylation and transactivation of p65 [313]. Treatment of IKK-16 or SC-514 significantly reduced HIV-1 replication in BaPexposed U1 cells, which provides strong evidence that the viral replication occurred via the NF- $\kappa \mathrm{B}$ signaling pathway. To further verify our results, we monitored the

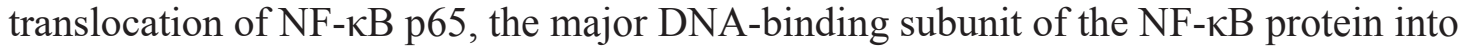
the nucleus, after treating the cells with the NF- $\mathrm{BB}$ inhibitors, IKK-16 and SC-514 and after siRNA silencing of CYP1A1. The knock-down of CYP1A1 in BaP-treated cells reduced the translocation of the NF- $\mathrm{B}$ p 65 unit into the nucleus, which shows an association of CYP1A1 with NF- $\kappa B$-mediated viral replication. As expected, the expression of the NF- $\kappa \mathrm{B}$ p65 protein was also observed to be lower in the nucleus of BaP-treated cells, after treatment with IKK-16 and SC-514, which further confirms that $\mathrm{BaP}-$ mediated HIV-1 replication occurs via the NF- $\kappa \mathrm{B}$ signaling pathway. Our findings reconfirm the role of ROS in the activation of NF- $\kappa B$ and its subsequent impact on HIV-1 gene transcription $[147,314]$. 
In addition to the ROS generated via the CYP-mediated metabolism of $\mathrm{BaP}$, there are other factors such as HIV-1 proteins whose contribution to ROS production cannot be ruled out. HIV-1 proteins such as tat, gp120, Nef, and Vpr are known to cause oxidative stress in the infected cells $[150,151,286,315,316]$. These HIV-1 proteins generate ROS via different mechanisms: tat via upregulation of spermine oxidases [317] or by activating the NADPH oxidase pathways [318]; gp120 via upregulation of CYP2E1, NADPH oxidases [286], and proline oxidase [319]; Vpr via interaction with adenine nucleotide translocator or NADPH oxidases 70; and Nef via direct interaction with NADPH oxidases [320]. Moreover, some of these viral proteins, such as tat and nef, are also known to enhance HIV-1 replication by interacting directly or indirectly on the LTR of the viral DNA [321].

\section{Conclusion}

In conclusion, the present study suggests NF- $\kappa \mathrm{B}$ activation through ROS generated via CYP1A1-mediated activation/metabolism of $\mathrm{BaP}$ as a novel pathway to explain smoking-mediated HIV-1 replication in monocytes-derived macrophages. This study has clinical relevance because the outcomes obtained from this study provide potential targets such as CYPs, oxidative stress, and NF- $\kappa \mathrm{B}$ signaling pathways, for developing novel interventions to improve treatment strategies for HIV-1-infected smokers. We have demonstrated that antioxidants such as vitamin $\mathrm{C}$ and $\mathrm{E}$, resveratrol, and pinostilbene, and CYP1A1 inhibitors namely ellipticine are capable of neutralizing the oxidative stress induced by $\mathrm{BaP}$ and subsequent viral load. These antioxidants and CYP1A1 inhibitors, which are chemodietary agents, have the potential to effectively control the viral replication in HIV-1-infected individuals who smoke tobacco. Furthermore, reduction of the viral load in these cells by targeting CYPs and the oxidative stress pathway may be beneficial in treating HIV-1-infected CNS cells. 


\section{CHAPTER 6. EXOSOMES FROM HUMAN PAPILLOMA VIRUS-INFECTED CERVICAL CANCER CELLS ENHANCE HIV-1 REPLICATION IN MACROPHAGES}

\section{Introduction}

HIV-1-infected women have a high prevalence of human papilloma virus (HPV), particularly type 16 and 18 infection that causes cervical cancer [322, 323]. Epidemiological data suggests that the incidence of cervical cancer in HIV-1-infected women is approximately 5- to 10-times higher compared to the uninfected women [324]. Cervical cancer has also been categorized into AIDS-defining cancer because of its specific higher prevalence in HIV-1-infected patients [325]. HIV-1 increases HPV infectivity by disrupting the tight junctions surrounding the epithelial cells. HIV-1 further enhances the ability of HPV to develop precancerous lesions and ultimately cervical cancer by promoting immunosuppression and upregulation of HPV oncogene [324]. However, whether HPV infection can have a reciprocal effect on HIV-1 pathogenesis is largely unknown. Therefore, there is a need to examine the biological interactions among HPV and HIV-1 to find a better preventive and treatment strategies to reduce HIV-1 pathogenesis in HIV-1/HPV coinfected patients

Evidences from previous reports show that cervical cancer cells constantly undergo oxidative stress $[210,326,327]$. The clinical samples from the cervical cancer patients have revealed higher ROS level, higher oxidative damage, and lower level of antioxidants, compared to the samples from healthy individuals [328-330]. In vitro experiments have also suggested a high level of ROS, profound downregulation in the genes associated with antioxidant proteins such as superoxide dismutase 1 (SOD1), SOD2, SOD3, peroxiredoxin 1 (PRDX1), PRDX2, glutathione S-synthetase (GSS), and glutathione peroxidase 6 (GPX6), and high presence of Poly [ADP-ribose] polymerase 1 (PARP1), a marker of oxidative stress-induced DNA damage in cervical carcinomaderived Caski cells [210]. HPV oncoproteins E6 and E7 stimulate the degradation of tumor-suppressor p53 protein [331], causing uncontrolled cell growth. p53 protein, which primarily has a function in apoptosis and genomic stability, is also reported to have an antioxidant role [332]. Downregulation of p53 suppresses its antioxidant potential and renders the cells vulnerable to oxidative damage. Furthermore, the expression of HPV E6 and E7 oncoproteins alone is sufficient to cause oxidative stress. Marullo et al. (2015) have demonstrated that HPV E6 and E7 proteins generate chronic oxidative response in the host cells via NADPH oxidase 2 (NOX2) activation [134]. E6 expression also downregulates SOD2 and glutathione peroxidase [134]. The suppression of antioxidant activity of these antioxidants could be another mechanism by which HPV generates ROS in the host cells.

Similar to other cells, HPV-infected cervical cells also secrete small nanosized extracellular vesicles (30-150 $\mathrm{nm}$ in size) called exosomes [333, 334]. Exosomes originate from the invagination of the lumen of the early endosomes. As the endosomes reach the later phase of their development, these invaginations bud off forming hundreds 
of intraluminal vesicles. These vesicles are released into the extracellular matrix of the cells through fusion into the cell membrane [335]. Exosomes carry wide variety of cellular proteins, lipids, mRNA, miRNA, and DNA from their originating cells to other cells via blood circulation. The role of exosomes in intracellular communication and transport has been well supported by many studies [336]. In HPV-infected cells, the virus hijacks the host exosomal system, thereby modulating both the contents and amount of exosomes released from HPV-infected cervical cells [334, 337]. The exosomes from the HPV-infected cervical cells are known to transport various cellular factors to the recipient cells $[337,338]$. It is possible that HPV-infected cells may be releasing oxidative stress factors into exosomes and transferring them to other cells via blood stream.

Oxidative stress is known to enhance the frequency of integration and replication of HPV genome [327, 339, 340]. ROS enhance HPV DNA integration by making a space for the viral genome to integrate into the host DNA through DNA damage in the host cells [339]. Oxidative DNA damage and the HPV oncoproteins E6 and E7 enhance the viral amplification by activating the viral transcriptional and replication proteins, E1 and E2 [327]. Oxidative stress-induced viral replication has also been observed in HIV-1 infection $[149,154]$. We recently demonstrated that oxidative stress generated via CYPmediated metabolism of benzo(a)pyrene $(\mathrm{BaP})$ triggered $\mathrm{HIV}-1$ replication in monocytes and macrophages [341]. We showed that ROS thus generated, activated the nuclear transcription factor NF- $\mathrm{B}$, thereby inducing transcription of the HIV-1. In the current study, we have introduced exosomes as a mediator for intracellular communication between HPV-infected cervical cancer cells and macrophages latently infected with HIV1. Our findings demonstrate that cervical cancer cells release exosomes containing oxidative stress factors into HIV-1-infected macrophages, where they induce HIV-1 replication.

\section{Materials and Methods}

\section{Cell Culture and Treatment}

Caski cells. Caski cells are human cervical cancer cell lines containing about 600 copies of HPV-16 genome per cell as well as sequences related to HPV-18 (ATCC). We cultured the Caski cells in Roswell Park Memorial Institute (RPMI) 1640 media containing 10\% Fetal bovine serum (FBS) and penicillin. We seeded about 2.5 million cells in a T-75 flask, cultured them for 3 days and collected the culture supernatant on the 4th day. For exosome isolation, we cultured the Caski cells in an RPMI media containing $10 \%$ exosome-free FBS and penicillin.

U1 cells. We cultured U1 cells as described in Chapter 5 and treated them with CCS and CCS-Exo for 3-4 days. 


\section{Exosome Isolation and Characterization}

We isolated exosomes from Caski cell culture media using ultracentrifugation method and Invitrogen-Total Exosome Isolation (from cell culture media) kit (Life Technologies, NY). For ultracentrifugation method, we first centrifuged at a lower speed $300 \mathrm{~g}$ followed by $2,000 \mathrm{~g}$ for 10 minutes each, which removed live cells and dead cells respectively. Further we centrifuged at $10,000 \mathrm{~g}$ for 30 minutes to remove cellular debris and microvesicles. Finally, we obtained exosomal pellet after centrifugation at 100,000$200,000 \mathrm{~g}$ for 70 minutes [342]. We followed the manufacturer's protocol to isolate exosomes using Invitrogen-Total Exosome Isolation kit. The cell culture supernatant was centrifuged at $2000 \mathrm{~g}$ for 30 minutes to remove any cellular debris present. Total exosome isolation reagent was added to the cell culture supernatant in a 1:2 ratio and the mixture was incubated overnight at $2^{\circ}-8{ }^{\circ} \mathrm{C}$. Next day, the mixture was centrifuged at $10,000 \mathrm{~g}$, $2^{\circ}-8{ }^{\circ} \mathrm{C}$ for 1 hour, to obtain the exosomal pellets. Although ultracentrifugation method yields exosomes of high purity, it is tedious and consumes $>10$-times media. The exosomes we obtained from Invitrogen-Total Exosome Isolation kit had purity almost equivalent to that obtained from the ultracentrifugation and the method takes less effort and reagent. Therefore, we used Invitrogen-Total Exosome Isolation kit for isolating exosomes for all our experiments. Western blotting was performed using antibodies against specific exosomal markers such as CD63 and CD81 to confirm the identity of exosomes. The exosomes were further characterized by measuring their size using zeta sizer as described previously.

\section{Viral Load}

We collected the supernatant from U1 cells treated with Caski cell supernatant or Caski cell-derived exosomes. HIV-1 viral p24 antigen level was measured in the U1 cell culture supernatant to access the viral load, using HIV-1 p24 Antigen ELISA kit (Zeptometrix Corporation, Buffalo, NY). We followed the manufacturer's protocol to perform the ELISA. The principle behind the p24 ELISA assay has been explained in Chapter 5.

\section{Measurement of ROS}

ROS was measured in U1 cells after CCS or CCS-Exo treatment. ROS was measured in the treated cells using the method described in Chapter 4. We used NovoCyte flow cytometer (ACEA, Biosciences Inc., San Diego, CA) to measure ROS and NovoExpress software to analyze the data. The background fluorescence signal due to unlabeled cells was reduced and only the live healthy patch of cells was gated to calculate the mean fluorescence intensity of the dye. 


\section{Total Antioxidant Capacity}

We measured antioxidant capacity of the CCS or CCS-exo treated U1 cells using Total Antioxidant Capacity Assay Kit (Bio Vision, Milpitas, CA). The assay was performed according to the manufacturer's protocol and as described previously [211].

\section{Cytotoxicity}

We used Pierce TM LDH cytotoxicity assay kit (Thermo Scientific, Rockford, IL) to measure the cytotoxicity in U1 cells after the exposure of Caski cell exosomes. We followed the manufacturer's protocol to conduct the assay. We performed the assay on culture supernatant obtained after the treatment. Dead cells release LDH enzymes from the cytosol into the culture supernatant through their damaged plasma membrane. The measure of LDH in the culture supernatant is proportional to the cytotoxicity suffered by the cells. The $\mathrm{LDH}$ kit is comprised of $\mathrm{LDH}$ reaction mixture containing lactate, $\mathrm{NAD}^{+}$, diaphrose, and terazolium salt. A catalytic reaction between the LDH in the medium and the reaction mixture generates a red color product, formazan, whose absorbance at 490 $\mathrm{nm}$ is directly proportional to the amount of $\mathrm{LDH}$.

\section{Apoptotic DNA Damage}

We observed the DNA damage of the CCS or CCS-Exo treated U1 cells under fluorescent microscope using the Apoptag ${ }^{\circledR}$ Iso Dual Fluorescence Apoptosis Detection kit (Millipore Sigma, Burlington, Massachusetts). We followed the manufacturer's protocol to perform the assay.

\section{RNA and Protein Isolation}

We used the method described in Chapter 5 to isolate RNA and protein from the cells.

\section{Quantitative Reverse Transcriptase Polymerase Chain Reaction (RTPCR)}

We used RTPCR to calculate the relative mRNA fold expression level of CYPs and AOEs in U1 cells after treatment of Caski supernatant/Caski-derived exosomes. The mRNA expression was measured following the method described as earlier in Chapter 4. We used the following specific TaqMan TM probes (CYP1A1 (Hs01054794_m1), CYP2A6 (Hs00430021_m1), SOD1 (Hs00533490_m1), SOD2 (Hs00167309_m1), catalase (Hs00156308_m1), and PRDX6 (Hs00705355_s1)) and GAPDH as an endogenous control. 


\section{Western Blotting}

We used Western blotting to identify the presence of CYPs, antioxidants, and HPV proteins in Caski-derived exosomes and to calculate the relative protein fold expression level of CYPs and AOEs in U1 cells upon treatment with Caski supernatant/Caski-derived exosomes. We performed western blotting as described in Chapter 4. We used the following primary antibodies: GAPDH Rabbit Mab, 1:2000 dilution, Cell Signaling Technology, Danvers, MA; CYP1A1 rabbit Mab, 1:200 dilution; CYP2A6 Mouse Mab, 1:200 dilution, Abcam, Cambridge, MA; SOD1 Mouse Mab, 1:1500 dilution; SOD2 Mouse Mab, 1:500 dilution; Catalase Mouse Mab, 1:1200 dilution, Santa Cruz Biotechnology. Inc., Dallas, TX; PRDX6 Rabbit Mab, 1:500 dilution, LifeSpan Biosciences, Inc., Seattle, WA; CD63, Rabbit Pab, 1:200 dilution, Proteintech Group, Inc Rosemont, IL). GAPDH and CD63 were used as loading controls for cellular and exosomal proteins, respectively.

\section{Exosome Uptake}

The CCS-Exo were labelled with GFP protein using Exo-GlowTM Exosome Labeling Kits (System Biosciences, CA) and as described previously [211]. Exosomal pellet containing $\sim 42 \mu \mathrm{g}$ of protein in $500 \mu \mathrm{l}$ of $1 \mathrm{X}$ PBS was incubated with $50 \mu \mathrm{l}$ of $10 \mathrm{X}$ Exo-Green dye at $37^{\circ} \mathrm{C}$ for 30 minutes. The labelling reaction was stopped by adding $100 \mu \mathrm{l}$ of ExoQuick-TC, provided along with the kit. The labelled CCS-Exo were exposed to U1 cells for 6 hours and their uptake was monitored under fluorescent microscope and flow cytometry.

\section{Statistical Analysis}

Statistical analyses were performed using Graphpad Prism 7.0 (La Jolla, CA). All data are presented as Mean \pm SEM of 3-5 independent experiments. Student's t-test or one-way ANOVA were used to calculate the statistical differences between the control and the treated groups, where appropriate. Significant difference was considered at $\mathrm{p}<0.05$.

\section{Results}

\section{Cell Culture Supernatant from Caski Cells Enhances Oxidative Stress and Viral Load in U1 Cells}

The exposure of CCS for 4 days significantly ( $\mathrm{p}$-value $=0.0084, \mathrm{n}=5$ ) increased the viral load in U1 cells by approximately 1.7-fold compared to the control (untreated cells) (Figure 6-1A). ROS acts as a secondary messenger for inducing HIV-1 replication in cells latently infected with HIV-1 [281]. To determine whether the viral replication is 
Figure 6-1. Caski cell culture supernatant (CCS) increases HIV-1 replication and oxidative stress in $\mathbf{U} 1$ macrophages.

(A). U1 macrophages were treated with $250 \mu 1$ of CCS every 24 hours for 4 days. p24

ELISA was performed on the supernatant obtained from the treatment to measure the viral load. To minimize high standard deviation of the mean due to variability in absorbance values in different experiments, we converted the control absorbance values to $100 \%$ and normalized the values of treated groups to $\%$ of the control. (B). ROS was measured in U1 macrophages after the 4 days exposure of CCS. The treated cells were incubated with $\mathrm{H}_{2}$ DCFDA, the fluorescence of which was monitored at maximum excitation and emission spectra of $495 \mathrm{~nm}$ and $529 \mathrm{~nm}$ respectively, using flow cytometer. (C). Figure C shows the graphical representation of ROS increase in CCStreated U1 cells (red graph) versus control cells (grey graph). X-axis represents mean fluorescence intensity (MFI), showing ROS level. (D). Total antioxidant capacity of the cells were measured in CCS-treated cells using Total antioxidant capacity assay kit. The values on $\mathrm{Y}$-axis represents the total amount of reduced $\mathrm{Cu}^{+}$in $\mathrm{nmol} / \mu \mathrm{l}$, which quantitatively gives the measure of antioxidant capacity of the cells. (E). Cytotoxicity after CCS exposure was measured using Pierce TM LDH cytotoxicity assay kit. The values on $\mathrm{Y}$-axis represent the absorbance values of formazan dye at $490 \mathrm{~nm}$, which gives the measure of cytotoxicity. The mean absorbance is obtained by subtracting the background absorbance at $680 \mathrm{~nm}$. All the data were obtained from the mean of at least three independent experiments with the error bars representing standard error of mean. Significant difference was considered at $\mathrm{p}<0.05$. $* * * * * *$ represents $\mathrm{p}<0.05, \mathrm{p}<0.005$ and $p<0.0005$ respectively. (F). Apoptotic DNA damage was examined using Apoptag ${ }^{\circledR}$ Iso Dual Fluorescence Apoptosis Detection kit. DAPI, FAM and CR590 dyes were used to stain the nucleus, DNase II and I-type of DNA breaks respectively. 

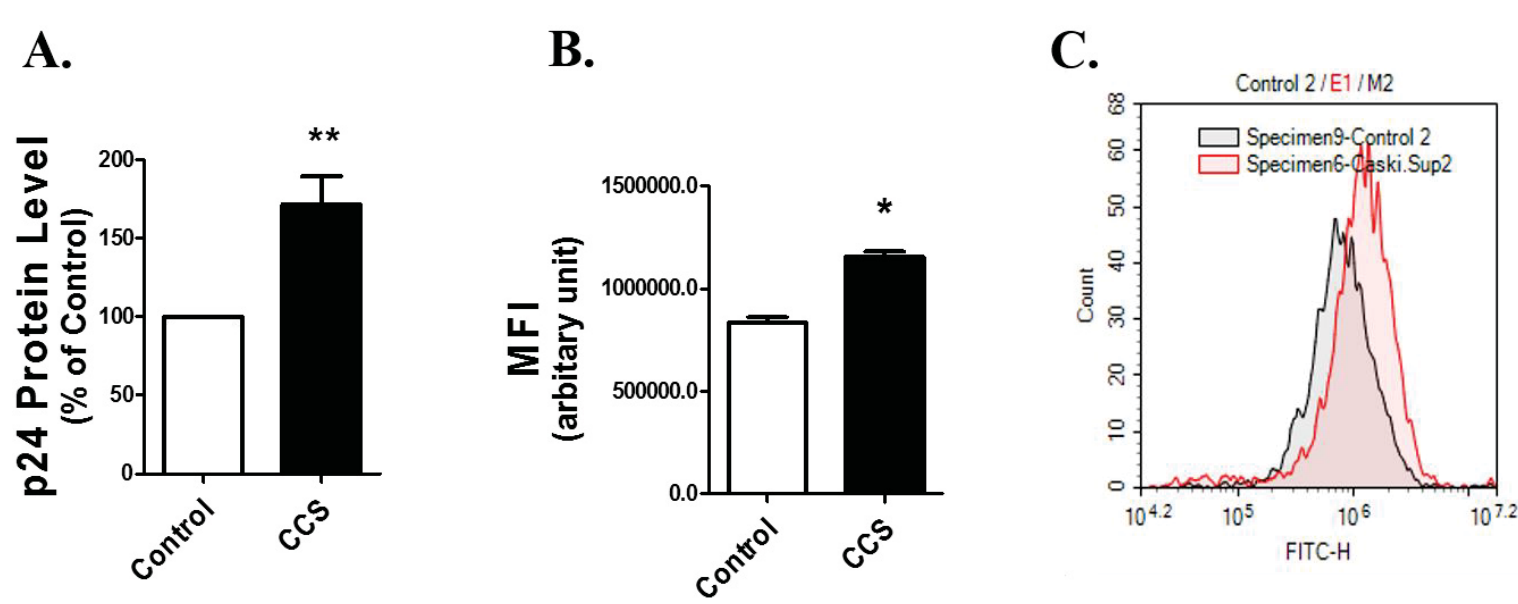

D.

E.

F.
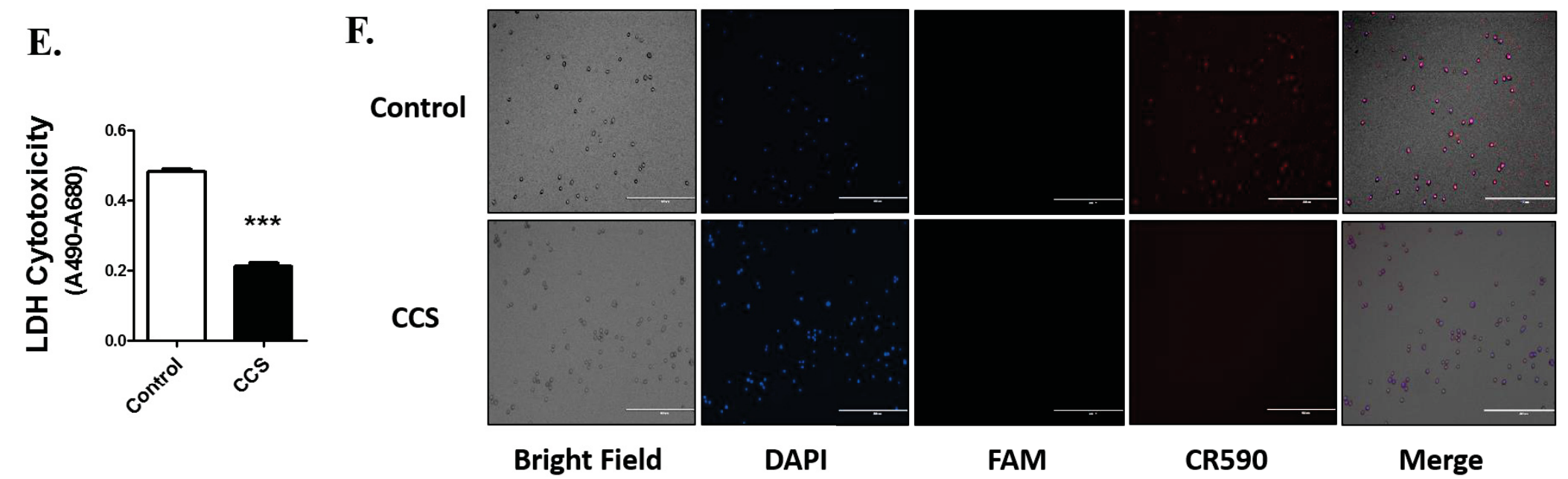
associated with oxidative stress, we measured the levels of ROS in the CCS-treated U1 cells. Our results showed that 4 days exposure of CCS induces ROS by $\sim 1.25$-fold (pvalue $=0.0169, \mathrm{n}=3$ ) in U1 cells (Figure 6-1B). Figure 6-1C shows the graphical representation of the ROS measurements shown in Figure 6-1B. The graph on

Figure 6-1C is also consistent with the result in Figure 6-1B. During the oxidative stress, cells employ antioxidant enzymes and proteins to neutralize the excess ROS, which may eventually wear away the total antioxidant capacity of the cells. Therefore, we monitored antioxidant capacity of U1 cells after 4 days of CCS treatment using total antioxidant capacity (TAC) assay kit. The TAC assay kit comprises of $\mathrm{Cu}^{2+}$, which is reduced by antioxidant molecules/proteins in the cell supernatant into $\mathrm{Cu}^{+}$. Although not significant, the data presented in Figure 6-1D shows a trend in decrease in the total antioxidant capacity of the CCS-treated cells compared to the control.

Next, we were interested to examine whether the excessive ROS induces cellular toxicity and DNA damage after CCS treatment. The cytotoxicity depicted in Figure 6-1E was performed using LDH assay. Interestingly, the 4 days treatment of CCS significantly ( $p$-value $<0.0001, n=6$ ) decreased the cytotoxicity in U1 cells by $\sim 60 \%$ (Figure 6-1E). To further confirm this result, we monitored apoptotic DNA fragmentation in the CSCtreated U1 cells. Figure 6-1F shows the fluorescent images of control and CCS-treated U1 cells labelled with DAPI, FAM, and CR590 dyes, which were used to stain the nucleus, DNA fragmentation with DNase Type II, and Type I ends, respectively. DNA fragmentation with Type I ends, commonly observed in most cells, occurs within the nucleus, which is an indicative of apoptosis by self-driven cell disassembly. In contrast, DNA fragmentation with Type II ends occurs in lysosomes of the phagocytes, where they eliminate the remains of apoptotic bodies [343]. The merged panel of Figure 6-1F shows reduced fluorescence intensity for CR590 in CCS-treated U1 cells compared to the control, which suggests that treatment of CCS protects U1 cells from DNase I type of DNA break. We did not observe any signal with FAM dye in both the control and treated cells, which suggests the absence of DNase II type of DNA break.

\section{Cell Culture Supernatant from Caski Cells Induces CYP Expression in U1 Cells}

CYP enzymes are known to generate ROS as byproducts, while metabolizing various endogenous and exogenous substances within the cells. Therefore, we monitored the mRNA and protein expression of CYPs (CYP 1A1 and 2A6) in U1 cells after 4 days of exposure of CCS. We particularly examined the expression of CYP $1 \mathrm{~A} 1$ and 2A6, because these CYPs are significantly expressed in U1 cells. The 4 days exposure of CCS significantly increased the expression of CYP1A1 at the mRNA level ( $\mathrm{p}$-value $=0.0367$, $\mathrm{n}=3$ ) (Figure 6-2A) and but not at the protein level (Figure 6-2B). The treatment also increased the protein expression of CYP2A6 in U1 cells significantly ( $p$ - value $=0.0647$, $\mathrm{n}=3$ ) (Figure 6-2D). However, there was no significant change in the expression of CYP2A6 at the mRNA level. 
A.
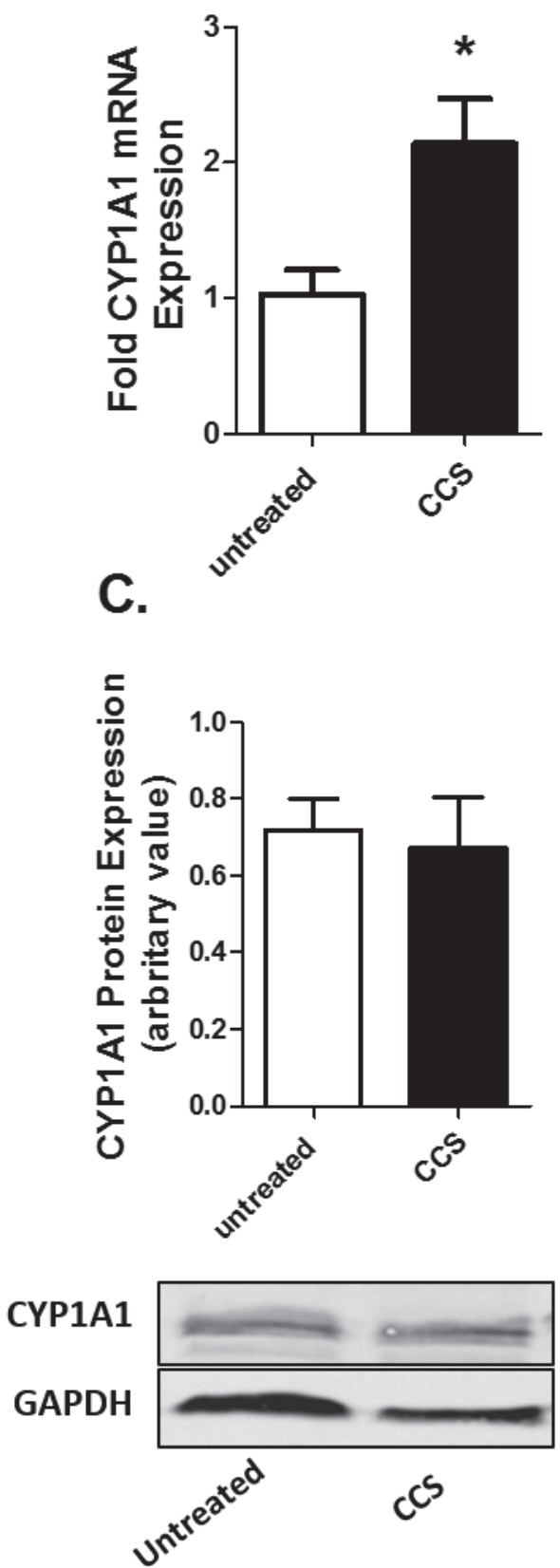

B.

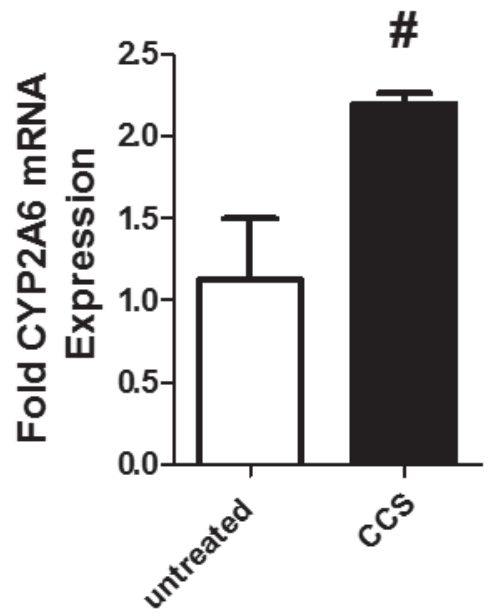

D.
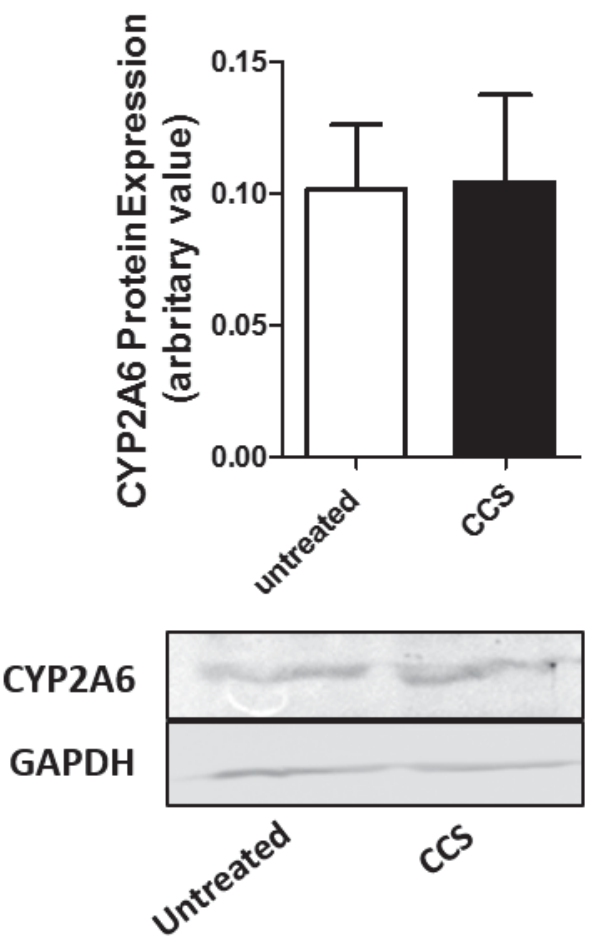

Figure 6-2. Caski cell culture supernatant (CCS) enhances CYP expression in U1 macrophages.

U1 macrophages were treated with $250 \mu 1$ of CCS every 24 hours for 4 days. Expression of CYP1A1 and CYP2A6 at mRNA (A and B) and protein (C and D) level were monitored in the treated cells. Data were obtained from the mean of at least three independent experiments with the error bars representing standard error of mean. Significant difference was considered at $\mathrm{p}<0.05$, which is represented by $*$. 


\section{Cell Culture Supernatant from Caski Cells Have No Significant Effect on AOEs Expression in U1 Cells}

AOE expression is expected to rise at the time of oxidative stress as a cellular response to combat the resulting oxidative damage. Therefore, we monitored the mRNA and protein expressions of major AOEs (SOD1, SOD2, catalase, and PRDX6) in U1 cells after 4 days exposure of CCS. The 4 days exposure of CCS did not have any significant effect on the expression of the AOEs at both the mRNA as well as protein level (Figure 6-3).

\section{U1 Cells Uptake CCS-derived Exosomes Containing Oxidative Stress Factors}

Since exosomes are one of the major factors that are secreted in the media from a variety of cells and are gaining attention in intercellular communication, we isolated and characterized exosomes secreted from the Caski cells. Recently, we reported isolation and characterizations of exosomes from the media of monocytic cells [211], and in this study we essentially used similar techniques. We characterized these exosomes with CD63 and CD81 (commonly used exosomal marker) in the CCS-derived exosomes (CCS-Exo) (Figure 6-4A) and size ( 100 nm) (data not shown). Our western blot images also showed the expression of CYP enzymes (CYP 1A1, 1B1 and 2A6), SOD1 and HPV 16 oncoprotein E6 in CCS-derived exosomes (Figure 6-4A). To show that U1 cells uptake these exosomes, we first labelled the exosomes with GFP and monitored its uptake by U1 cells under fluorescent microscope and flow cytometer after 6 hours. The merged panel of Figure 6-4B shows higher GFP fluorescence in CCS-Exo treated U1 cells, compared to the untreated cells, indicating the exosome uptake by U1 cells. We further verified this result by measuring the GFP fluorescence using flow cytometer. In Figure 6-4C, we can see that the red graph (CCS-Exo treated U1 cells) shifts towards the far right compared to the grey graph (untreated U1 cells), indicating higher mean fluorescence intensity for the former and hence the uptake of CCS-Exo by U1 cells. Our observation is consistent with our previous study, where we observed uptake of U937 monocytes-derived exosomes by U1 cells in 6 hours [211].

\section{CCS-derived Exosomes Enhance Oxidative Stress and Viral Load in U1 Cells}

To examine if the exosomes in the CCS were responsible for the increase in viral load in U1 cells, we treated the CCS-Exo to the U1 cells. U1 cells were treated with exosomes isolated from $1 \mathrm{ml}$ of CCS (equivalent to $\sim 42 \mu \mathrm{g}$ protein) and cultured for 4 days. Exposure of the CCS-Exo for 4 days significantly ( $p$-value $=0.0007, n=6$ ) increased the viral load by approximately 1.3-fold (Figure 6-5A). CCS-Exo treatment significantly ( $\mathrm{p}$-value $=0.0236, \mathrm{n}=3$ ) increased the ROS level in U1 cells (Figure 6-5BC). Although the data was not statistically significant, we observed a decreasing trend in the total antioxidant capacity of the U1 cells after CCS-Exo treatment (Figure 6-5D). The increase in ROS and decrease in total antioxidant capacity both are indicative of 
Figure 6-3. Caski cell culture supernatant (CCS) alters antioxidant enzyme (AOE) expression in U1 macrophages.

U1 macrophages were treated with $250 \mu$ of CCS every 24 hours for 4 days. Expression of SOD1, SOD2, catalase and PRDX6 were monitored at mRNA (A-D) and protein (EH) levels in the treated cells. Data were obtained from the mean of at least three independent experiments with the error bars representing standard error of mean. Significant difference was considered at $\mathrm{p}<0.05$, which is represented by $*$ 
A.

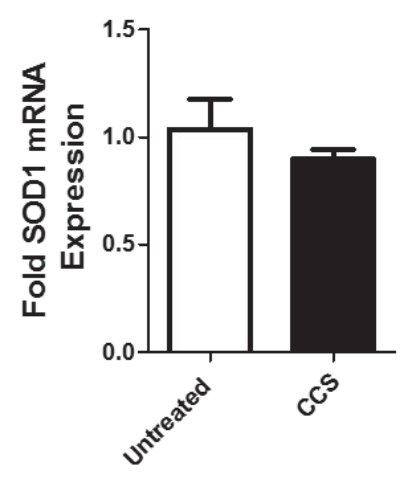

E.
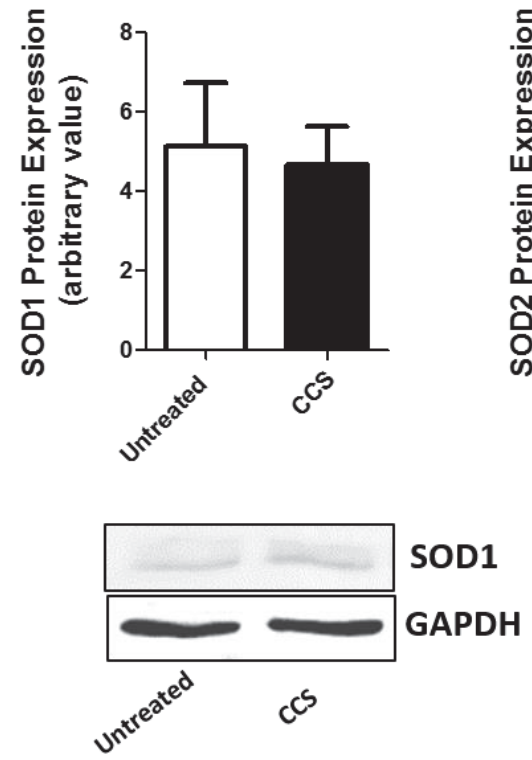

B.

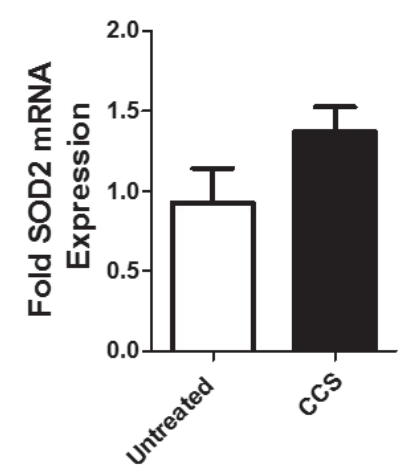

F.

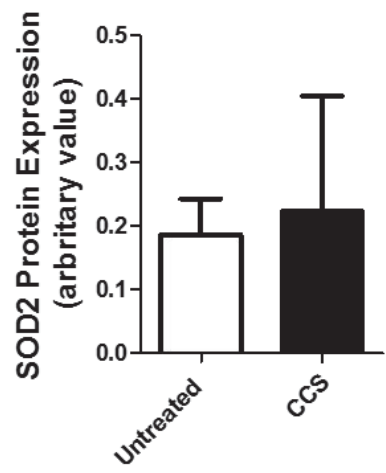

c.

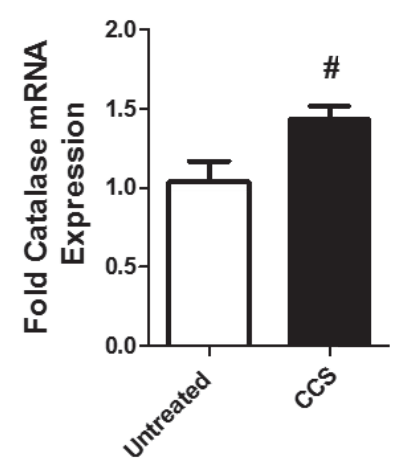

G.

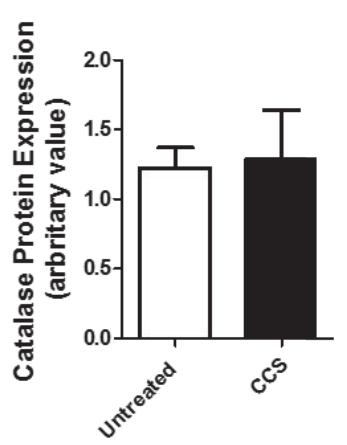

D.

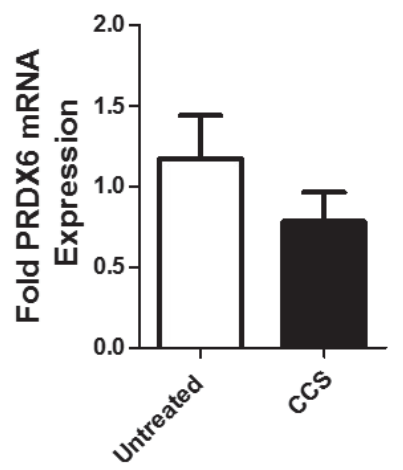

H.

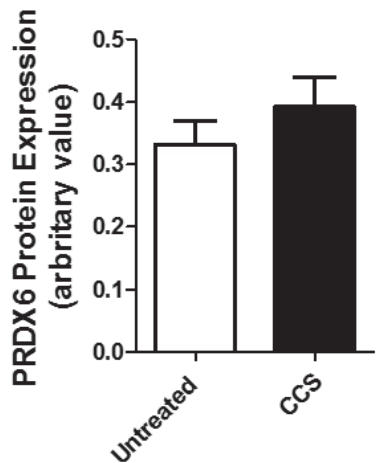

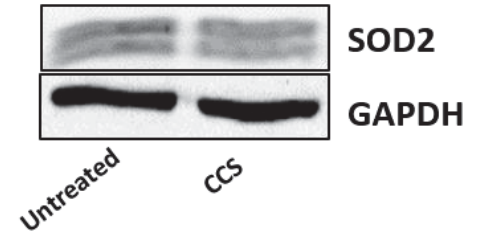
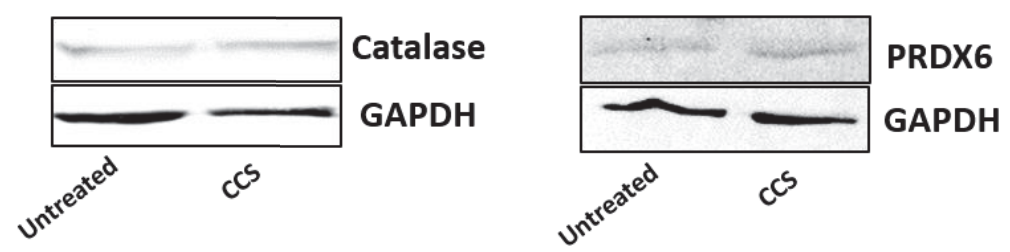
A.

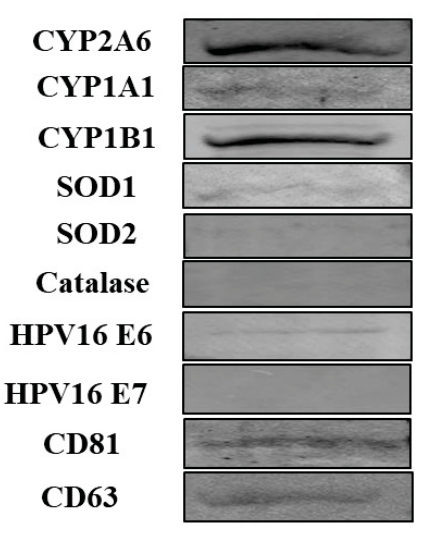

B.

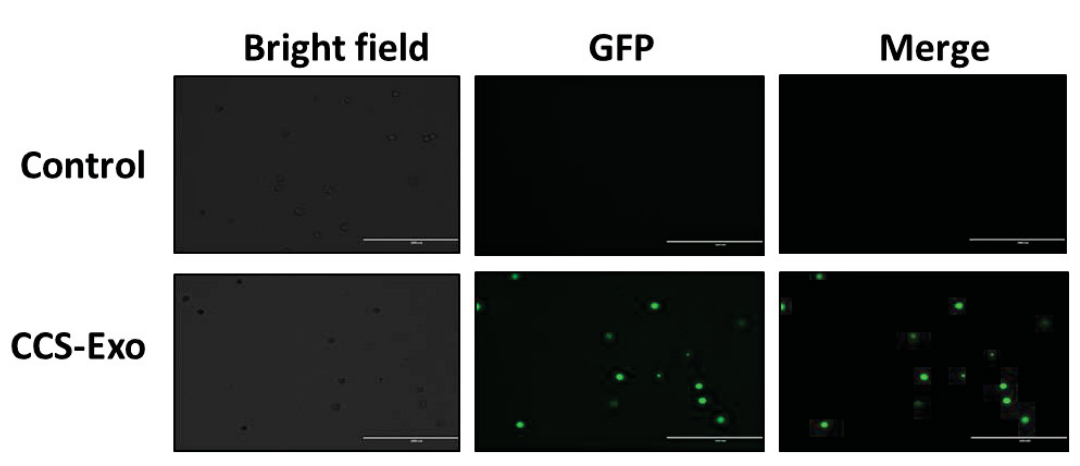

C.

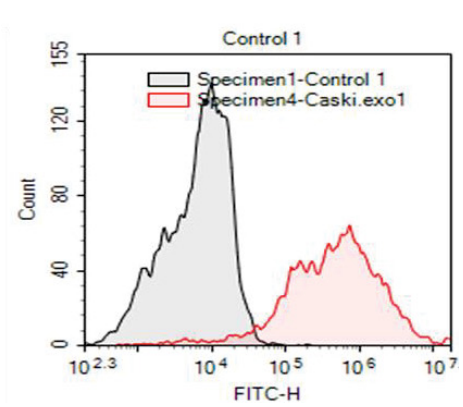

Figure 6-4. U1 macrophages uptake CCS-derived exosomes (CCS-Exo) that contain oxidative stress factors.

(A). The exosomal protein obtained from CCS-derived exosomes (CCS-Exo) were examined for the expression of exosomal marker, CD63, CYPs (1A1, 1B1, 2A6, 3A4) and AOEs (SOD1, SOD2, catalase, PRDX6) and HPV 16 oncoproteins (E6). Data not shown for the proteins which were absent. (B). To monitor the exosomes uptake by U1 cells, we labeled the CCS-Exo with GFP and treated the labelled exosomes to U1 cells. After 6 hours of incubation, we monitored the fluorescent intensity of GFP under fluorescent microscope. (C). The GFP fluorescence was further quantified using flow cytometry. 
Figure 6-5. CCS-derived exosomes (CCS-Exo) increases HIV-1 replication and oxidative stress in $\mathrm{U} 1$ macrophages.

(A). U1 macrophages were exposed with exosomes derived from $1 \mathrm{ml}$ of CCS $(\sim 42 \mu \mathrm{g}$ of exosomal protein) for 4 days. p24 ELISA was performed on the supernatant obtained from the treatment to measure the viral load. To minimize high standard deviation of the mean due to variability in absorbance values from different experiments, we converted the control absorbance values to $100 \%$ and normalized the values of treated groups to $\%$ of the control. (B). ROS was measured in U1 macrophages after the 4 days exposure of CCS-Exo. The treated cells were incubated with $\mathrm{H}_{2}$ DCFDA, the fluorescence of which was monitored at maximum excitation and emission spectra of $495 \mathrm{~nm}$ and $529 \mathrm{~nm}$ respectively, using flow cytometer. (C). Figure $\mathrm{C}$ shows the graphical representation of ROS increase in CCS-Exo-treated U1 cells (red graph) versus control cells (grey graph). $\mathrm{X}$-axis represents mean fluorescence intensity (MFI), showing ROS level. (D). Total antioxidant capacity of the cells was measured in CCS-treated cells using Total antioxidant capacity assay kit. The values on Y-axis represents the total amount of reduced $\mathrm{Cu}^{+}$in $\mathrm{nmol} / \mu \mathrm{l}$, which quantitatively gives the measure of antioxidant capacity of the cells. (E). Cytotoxicity after CCS exposure was measured using Pierce TM LDH cytotoxicity assay kit. The values on Y-axis represent the absorbance values of formazan dye at $490 \mathrm{~nm}$, which gives the measure of cytotoxicity. The mean absorbance is obtained by subtracting the background absorbance at $680 \mathrm{~nm}$. All the data were obtained from the mean of at least three independent experiments with the error bars representing standard error of mean. Significant difference was considered at $\mathrm{p}<0.05$. * $* * * * * *$ represents $\mathrm{p}<0.05, \mathrm{p}<0.005$ and $\mathrm{p}<0.0005$ respectively. (F). Apoptotic DNA damage was examined using Apoptag® Iso Dual Fluorescence Apoptosis Detection kit. DAPI, FAM and CR590 dyes were used to stain the nucleus, DNase II and I-type of DNA breaks respectively. 

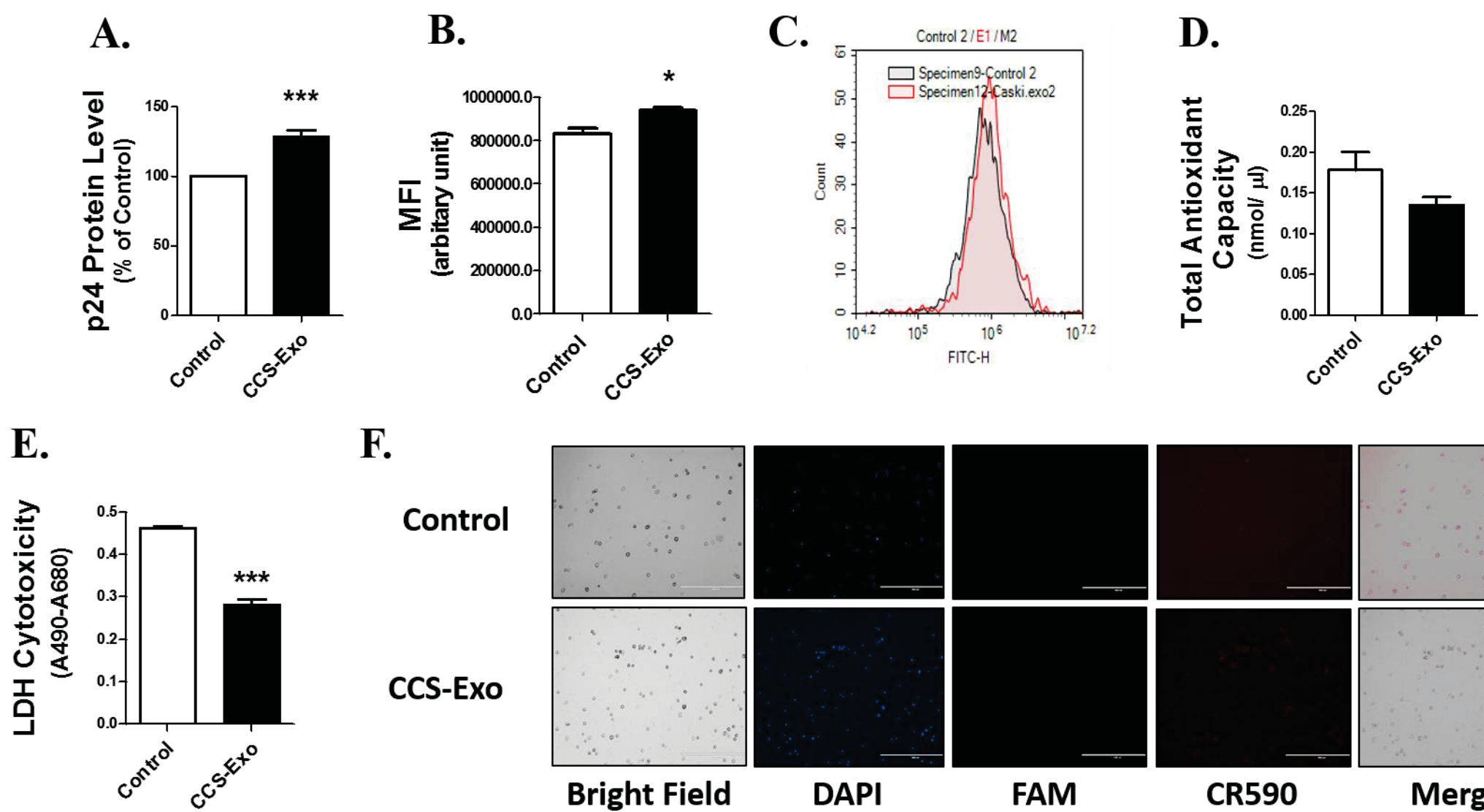

F.
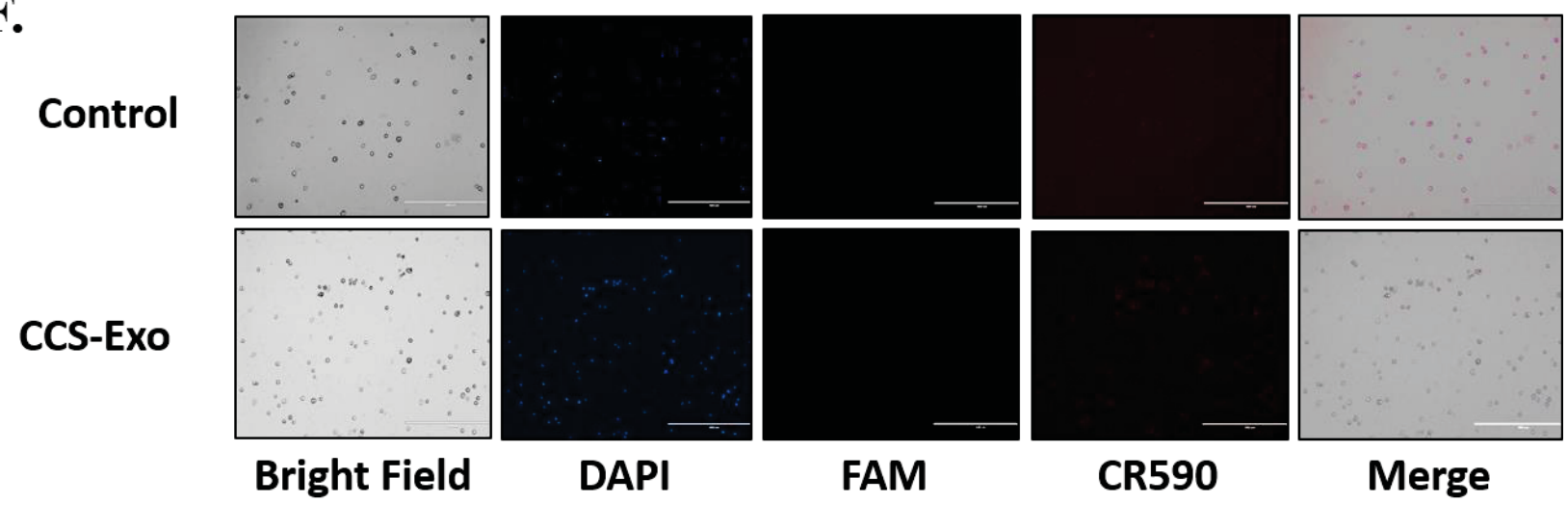
oxidative stress. Next, we monitored the cytotoxicity and DNA damage in CCS- Exo treated U1 cells. Interestingly, we observed $\sim 40 \%$ decrease ( $p$-value $<0.0001, n=6$ ) in cytotoxicity (Figure 6-5E) in the treated U1 cells. Similarly, the merged panel of Figure 6-5F shows lower CR590 fluorescence for the treated cells than for the control, indicating lower extent of DNA damage in the treated cells. These results are in agreement with the results from CCS-treated U1 cells.

\section{Treatment of Antioxidants, CYP-inhibitors, and Chemodietary Agents Reduce Viral Load in Caski Exosomes-treated U1 Cells}

To confirm that HIV-1 replication is occurring via a CYP-mediated oxidative stress pathway, we treated the CCS-Exo exposed U1 cells with an antioxidant, resveratrol, CYP1A1 inhibitor, ellipticine, and CYP2A6 inhibitor, tryptamine. These inhibitors are relatively selective for the individual CYPs. U1 cells were exposed to exosomes isolated from $1 \mathrm{ml}$ of CCS (comprising of $\sim 42 \mu \mathrm{g}$ of exosomal protein) for 4 days. To the CCS-Exo-treated U1 cells resveratrol $(25 \mu \mathrm{M})$, ellipticine (Epi, $1 \mu \mathrm{M})$, and Tryptamine (Tryp, $20 \mu \mathrm{M}$ ) were added every 24 hours for 4 days. $500 \mu$ l of exosome-free media was added after each treatment to ensure the healthy growth of the cells. Treatment of resveratrol ( $\mathrm{p}$-value $<0.0001, \mathrm{n}=3$ ), ellipticine ( $\mathrm{p}$-value $<0.0001, \mathrm{n}=3$ ), or tryptamine ( $\mathrm{p}$-value $<0.0001, \mathrm{n}=3$ ) significantly reduced the viral load in CCS-Exo exposed U1 cells, suggesting the role of CYP 1A1 and 2A6, and oxidative stress on viral load increase in U1 cells (Figure 6-6A-B).

Chemodietary agents such as curcumin and cucurbitacin-D are known to reduce cancer progression [344-346], as well as HIV-1 replication [347, 348] through their antioxidant potential. We treated Curcumin $(20 \mu \mathrm{M})$ and Cucurbitacin-D $(0.1 \mu \mathrm{M})$ to the CCS or CCS-Exo-treated U1 cells for 4 days, every 24 hours, with an assumption that they will combat the oxidative stress caused by CCS or CCS-Exo and hence reduce the viral replication in U1 cells. As expected, treatment of curcumin ( $p$-value $<0.0001, n=7$ ) and cucurbitacin-D ( $\mathrm{p}$-value $<0.001, \mathrm{n}=5$ ) significantly reduced the viral load in CCStreated cells (Figure 6-6C). We also observed significant decrease in viral load in CCSExo-treated U1 cells with curcumin ( $\mathrm{p}$-value $<0.01, \mathrm{n}=3$ ) or Cucurbitacin-D ( $\mathrm{p}$-value $<0.001, \mathrm{n}=3$ ) treatment (Figure 6-6D).

\section{Discussion}

There is a high risk and prevalence of HPV co-infection in HIV-1-infected individuals, which causes severe forms of cervical cancer. HIV-1 modulates the host cell microenvironment for HPV invasion, through destruction of tight junctions between the cells and immunosuppression [324]. However, it is unknown if there exists a cross-talk between HPV and HIV-1-infected cells. The current study highlights exosomes as a means of intracellular communication between the two cells. Here, we demonstrate for the first time that HPV-infected cervical cancer cells transfer oxidative stress factors 

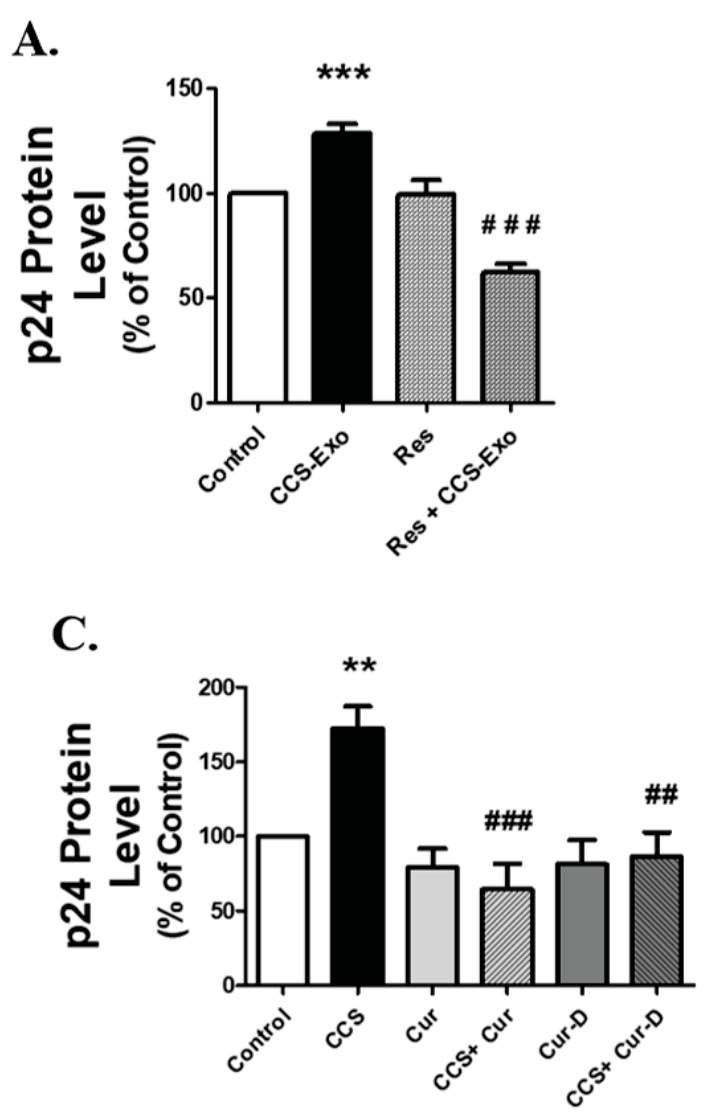

B.
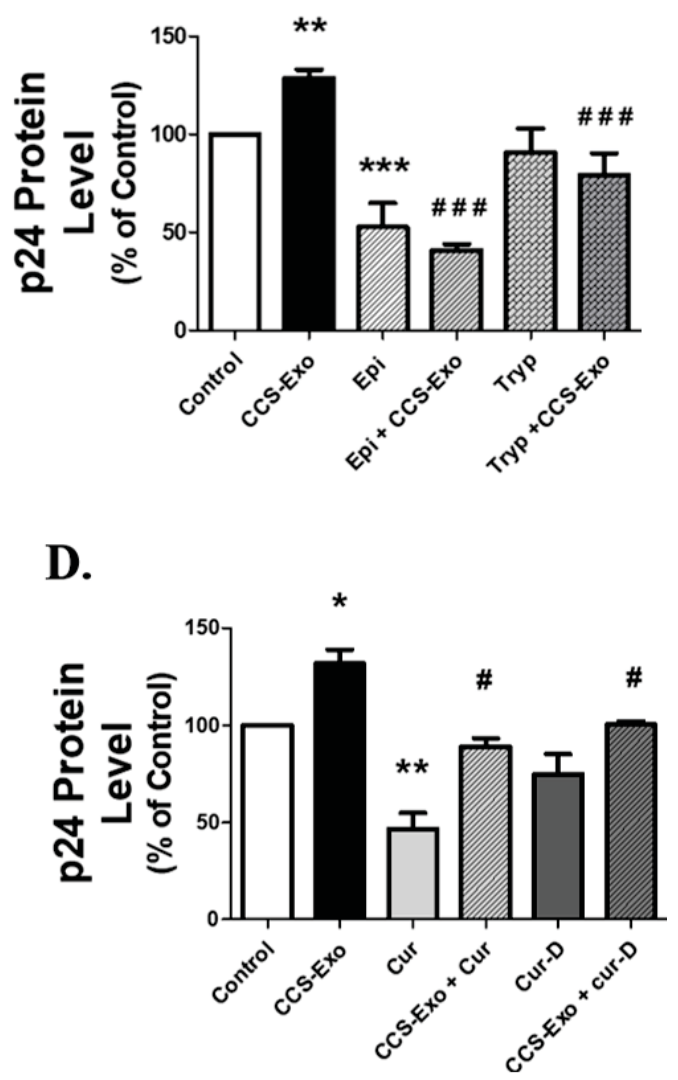

Figure 6-6. Treatment of antioxidant, CYP inhibitors, and chemodietary agents reduce CCS or CCS-derived exosomes (CCS-Exo)-induced HIV-1 replication in U1 macrophages.

To confirm the role of CYP-induced oxidative stress in CCS-Exo-treated U1 cells, we treated U1 macrophages with antioxidant, resveratrol (A. Res, $25 \mu \mathrm{M})$, CYP1A1 inhibitor, ellipticine (B. Epi $1 \mu \mathrm{M})$ and CYP2A6 inhibitor, Tryptamine (B. Tryp, $20 \mu \mathrm{M})$. We also treated the U1 cells exposed to CCS (C) or CCS-Exo (D) with curcumin (cur, 20 $\mu \mathrm{M})$ and cucurbitacin-D (Cur-D, $0.1 \mu \mathrm{M})$. All the data were obtained from the mean of at least three independent experiments with the error bars representing standard error of mean. Significant difference was considered at $\mathrm{p}<0.05$. *, ****** represents $\mathrm{p}<0.05$, $\mathrm{p}<0.005$ and $\mathrm{p}<0.0005$ respectively, when compared to the control. \#, \#\#, \#\#\# represents $\mathrm{p}<0.05, \mathrm{p}<0.005$ and $\mathrm{p}<0.0005$ respectively, when compared CCS or CCS-Exo groups. 
(such as CYPs and HPV proteins) via exosomes to the recipient HIV-1-infeceted monocytic cells to induce HIV-1 replication. The exosomal CYPs (CYP 1A1 and 2A6) exert an additive effect on the basal CYPs of the recipient cells. The cumulative CYPs promote the metabolism of endogenous substances, thereby generating massive ROS, which eventually exacerbate the HIV-1 replication in the recipient cells (Figure 6-7). Our study also suggests a treatment strategy for HIV-1-infected patients who are also infected with HPV by using chemodietary agents.

In the current study, we have shown the interaction between HPV and HIV-1 infected cells using Caski cells and U1 cells, which are widely used cell models for HPV and latent HIV-1 infection. To determine the interaction between the Caski cells and U1 cells, we initially exposed the U1 cells with Caski cell culture supernatant (CCS). Exposure of CCS to U1 cells significantly increased oxidative stress and HIV-1 replication in U1 cells. Initially, we made an assumption that Caski cells released oxidative stress factors into the cell culture media, which when transferred to the U1 cells, induced oxidative stress and viral replication in U1 cells. Later, we were interested to investigate if Caski cells were communicating with the U1 cells via exosomes in the CCS. Therefore, we isolated exosomes from CCS and exposed them to U1 cells. Interestingly, exposure of CCS-derived exosomes (CCS-Exo) also revealed higher viral load and ROS level.

We mostly used the exosome isolation kit method for isolating exosomes from the CCS. Initially, we tried both ultracentrifugation and exosome isolation kit method to isolate exosomes. As we did not observe a significant difference in the yield and purity of the exosomes isolated from these methods, we opted to use the kit method because it is easy to use, consumes less time and is cost-effective. The presence of specific exosome markers such as CD63 and CD81 in the exosomal pellet obtained from CCS justifies its identity as exosomes. Moreover, in our previous work we have used extensive characterization methods to confirm the identity of exosomes from cell culture media [211]. Our results from transmission electron microscope and zetasizer have shown that the extracellular vesicles we isolated have size around $100 \mathrm{~nm}$, which is about the size of the exosomes; we also observed acetylcholinesterase activity in the isolated exosomes and the presence of several exosomal marker proteins such as alix, CD63, CD81 and Tsg101 in the exosomes.

ROS can activate cells latently infected with HIV-1 to produce new viruses via activation of nuclear transcription factor, NF- $\kappa \mathrm{B}$ [281, 341, 349]. ROS induces translocation of cytosolic NF- $\kappa \mathrm{B}$ subunits into the nucleus, where it binds to the core enhancer region of HIV-1 long terminal repeat (LTR) to induce HIV-1 transcription. ROS generated through CCS/CCS-derived exosomes could induce HIV-1 replication in the U1 cells via similar mechanism. Furthermore, redox state of the monocytes/macrophages are also reported to regulate the expression of $\mathrm{C}-\mathrm{C}$ chemokine receptor type 5 (CCR5) and C-X-C chemokine receptor type 4 (CXCR4), which are major coreceptors for HIV-1 entry into monocytes/macrophages [350, 351]. ROS elevation through CCS/CCS-derived exosomes could facilitate the viral entry and hence increased the viral load within the U1 cells via overexpression of these chemokine 


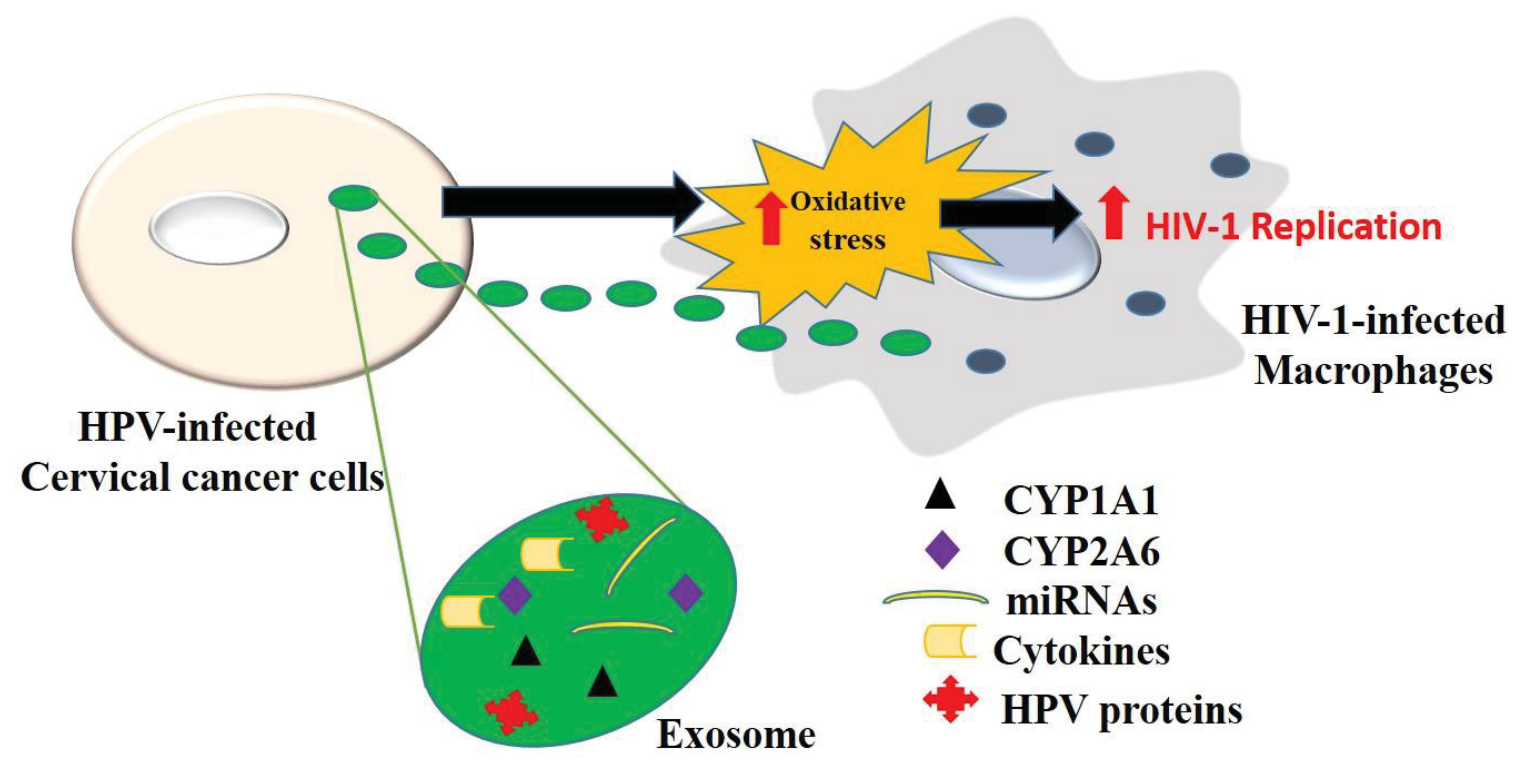

Figure 6-7. Proposed model for HPV-HIV-1 interaction via exosomes.

Exosomes from cervical cancer cells carry oxidative stress factors that exacerbates HIV-1 replication in HIV-1-infected macrophages. As a result of constant oxidative stress, cervical cancer cells package oxidative stress factors like CYP enzymes (CYP 1A1, 2A6, 1B1), miRNAs, cytokines, HPV oncoproteins etc. into exosomes and transfer them to cells, e.g. macrophages at distant sites. The exosomes then release the oxidative stress factors in recipient cells, which further enhances ROS production in the cells. The ROS thus generated contributes as a secondary messenger to trigger HIV-1 in macrophages. 
receptors. Treatment of antioxidants such as glutathione, glutathione ester, and N-acetylL-cysteine has been reported to suppress HIV-1 expression in U1 cells, which strongly supports the involvement of oxidative stress in HIV-1 expression in macrophages [352]. Our findings are consistent with the previous reports and they further elaborate CCS/CCS-derived exosomes as a novel source of ROS for HIV-1 replication in macrophages.

We observed that exposure of CCS/CCS-derived exosome decreased the cell death and DNA damage in U1 cells, despite the oxidative stress. Under the stressed condition, the cells initially attempt to protect themselves from the insult by activating signaling pathways that promote cell survival. In case they are unable to overcome the insult, they activate death signaling pathways [353]. In the current context, treatment of $\mathrm{CCS} / \mathrm{CCS}$-derived exosome is likely to activate cell survival pathways such as antioxidant defense system, heat shock and unfolded protein response, and DNA damage repair to rescue the U1 cells from the oxidative insult [353]. Furthermore, most of the cancer cells, including cervical cancer are known to overexpress and release antiapoptotic factors like survivin, which arrest apoptosis by inhibiting caspase activation $[354,355]$. It could be possible that protective mechanism is mediated by anti-apoptotic factors like survivin in the CCS-derived exosomes. In addition, HIV-1 protein Nef, is known to protect HIV-1-infected host cells by inhibiting apoptosis signal-regulating kinase 1 (ASK1) thus preventing Fas and TNF- $\alpha$-mediated apoptotic cell death [356]. HIV-1 could be promoting the cell survival in the treated U1 cells, so as to frame a favorable environment for its multiplication.

Next, we were interested in finding what oxidative stress factors are present in $\mathrm{CCS} / \mathrm{CCS}$-derived exosomes, and what mechanistic pathway they trigger to induce oxidative stress and consequently HIV-1 replication in U1 cells. Overexpression of CYPs are observed in several cancers, including cervical cancer [357] and CYPs are known to generate excessive ROS as a byproduct during the phase-I metabolism of various endogenous and exogenous substrates [304]. Taking this fact into consideration, we examined the effect of CCS in the expression of CYPs in U1 cells. We particularly examined the expression of CYP1A1 and CYP2A6 because these enzymes are expressed in monocytic cells. In addition to metabolize many endogenous compounds, CYP1A1 and CYP2A6 are the major enzymes for metabolizing polyaryl hydrocarbons and nicotine present in the cigarette smoke respectively $[155,273]$. As expected, we observed significant increase in the protein expression of both the CYPs, 1A1 and 2A6 in U1 cells after CCS exposure. The concurrent upregulation of CYPs and elevation in ROS levels signified that the oxidative stress in the U1 cells could be occurring via a CYP-mediated pathway. We did not observe any change in the expression of antioxidant enzymes after CCS exposure. However, there was a decrease in the total antioxidant capacity of the cells after CCS or CCS-mediated exosome treatment.

In this study, we observed increase in the expression of CYPs at the mRNA level but not at the protein level. We have observed this kind of discrepancy in our previous studies as well [154]. As mRNA is translated into protein, it is theoretically believed that, there is a correlation between the expression of gene at the mRNA and the protein level 
but in practical, it is not necessary that mRNA expression changes would reflect similar changes in the protein expression [358-360]. The reason for decrease in expression of the proteins, despite their increased mRNA expression could be due to various transcriptional and post-translational modifications, differential stability of mRNAs vs. proteins, or due to interference by different miRNAs.

Next, we verified the presence of CYPs 1A1, 2A6 and 1B1 in the CCS-derived exosomes, which further strengthens our assumption about involvement of CYP-pathway in CCS-induced oxidative stress. To further confirm the role of CYPs in oxidative stressinduced HIV-1 replication, we treated the U1 cells exposed to CCS-derived exosomes with resveratrol (an antioxidant), ellipticine (CYP1A1 inhibitor) and Tryptamine (CYP2A6 inhibitor). Treatment of these antioxidant and CYP inhibitors significantly reduced the viral load in the treated U1 cells. (role of CYP from CCS-derived exosomes using siRNA) Overall, our findings suggested that Caski cells transfer CYPs to the U1 cells via exosomes. Upon reaching the U1 cells, these exosomes release the CYPs into the cytosol where they induce oxidative stress and subsequently HIV-1 replication. Our observations are in agreement with our previous reports, where we have observed higher expression of CYPs, oxidative stress, and HIV-1 viral load in the plasma samples of HIV1 smokers, which shows the association of CYP-mediated oxidative stress pathway in HIV-1 replication [153]. Recently, we have confirmed this association in vitro in U1 cells where we demonstrated, CYP1A1 metabolizes benzo(a)pyrene (a harmful carcinogen in cigarette smoke), causing massive production of ROS and subsequently trigger HIV-1 replication in the U1 cells [341].

Apart from CYPs, we also observed the presence of other oxidative stressinducing factors such as HPV type16 oncoprotein E6 in the CCS-derived exosomes. HPV16 E6 and E7 oncoproteins are known to cause chronic oxidative stress in HPVinfected and uninfected cells via NOX2 activation [134]. Besides, CCS-derived exosomes may also contain various cytokines and miRNAs that may trigger HIV-1 replication in U1 macrophages. Cytokines such as, TNF- $\alpha$ triggers HIV-1 gene transcription through activation of NF- $\mathrm{kB}$; IL-6 increases the expression of viral proteins and RT; IL-6 together with TNF- $\alpha$ have a synergistic effect on HIV-1 replication [189]. Micro RNA (miRNA) 34a and miRNA 181 are reported to enhance HIV-1 viral load by inhibiting the cellular restriction factors (e.g., p21, TASK, and SAMHD1), which inhibits different stages of HIV-1 life cycle [361, 362]. Since, these miRNAs are also expressed in cervical cancer cells, it is possible that they are being transported to the host cells via exosomes, where they contribute to HIV-1 replication.

Curcumin and cucurbitacin-D are shown to inhibit cancer growth in cervix by inducing apoptosis and arresting cell cycle [344-346]. In addition to the anti-cancer property, curcumin and cucurbitacin have also shown to suppress HIV-1 pathogenesis through its antioxidant potential [347, 348]. Curcumin is shown to reduce HIV-1 transcription by inhibiting the HIV-1 protein, Tat-mediated LTR promotor transactivation [347]. Considering their potential antioxidant and antiviral properties, we examined the preventive effect of these agents to HIV-1 replication induced by CCS/CCS-derived exosomes. As expected, treatment of both the compounds significantly reduced HIV-1 
viral load induced by CCS or CCS-derived exosomes in U1 cells. Curcumin and cucurbitacin-D are known to scavenge hydroxyl and superoxide ions at higher concentration [363]. It is therefore possible that these compounds are inhibiting the viral replication by lowering the ROS level. Furthermore, the use of curcumin and Cucurbitacin-D as antioxidants to block the viral replication is clinically safer as these natural antioxidants are less carcinogenic compared to synthetic antioxidants, such as butylatedhydroxy toluene (BHT) and butylatedhydroxy anisole (BHA). Furthermore, the use of these chemodietary agents in HIV-1 patients with cervical cancer is more beneficial because of their dual effect on reducing cancer progression and HIV-1 replication.

\section{Conclusion}

In conclusion, we have demonstrated that cervical cancer cells exacerbate HIV-1 replication in monocytic cells via transferring oxidative stress factors such as CYPs and HPV oncoproteins through exosomes. We have also shown that the viral replication undergoes via a CYP-mediated oxidative stress pathway and it can be reduced by treatment of chemodietary agents like curcumin and Cucurbitacin-D. The present study therefore, provides scientific rationale for the development of novel therapeutic treatment for HIV-1 infected women with cervical cancer. 


\section{CHAPTER 7. CONCLUSIONS AND FUTURE DIRECTIONS}

With the introduction of ART since 1995, the number of AIDS-related deaths have significantly decreased and consequently, the number of PLWHA has increased [364]. With the increase in life-span, numerous pathological complications including cancers of various organs and tissues, neurological disorders and various opportunistic infections have been observed in PLWHA. ART has been able to control plasma HIV-1 levels and improve $\mathrm{CD}^{+}$cell counts to large extent, however, complete eradication of HIV-1 has not been achieved. One of the major hindrances to cure HIV-1 is the rapid formation of latent HIV-1 reservoirs in memory T cells, monocytes, and macrophages. The presence of these viral reservoirs is observed even in PLWHA on ART for lifelong. The latent HIV-1 in these reservoirs are activated to produce new viruses in presence of stress factors like cytokines and [133]infections [365] and exposure to various drugs of abuse [366]. Most of these stress factors use ROS as a secondary messenger to stimulate the nuclear transcription factors, NF- $\kappa \mathrm{B}$ and NFAT, which enhance the transcription of latent HIV-1 genome [149].

There is a high prevalence of drug abuse, especially tobacco smoking among PLWHA. Several components in cigarette smoke such as nicotine and polyaryl hydrocarbons are known to be metabolized by CYP enzymes through phase I metabolism. During the metabolism, CYPs generate ROS as intermediate products through various coupling and auto oxidation reactions. Therefore in project I, we initially proposed that ROS generated through CYP-mediated metabolism of cigarette smoke constituents, especially BaP, triggers HIV-1 replication in viral reservoirs such as monocytes and macrophages. Furthermore, PLWHA also suffer from coinfection by various pathogens due to immunosuppression by HIV-1. Among the various coinfections, high-risk HPV coinfection by genotype 16 and 18 that causes cervical cancer is highly prevalent in PLWHA. Infections by pathogens such as syphilis [367], mycobacterium tuberculosis [365], and hepatitis [368] have been shown to induce HIV-1 replication. Therefore in project II, we proposed that HPV may also trigger latent HIV-1 reactivation in infected monocytes/macrophages via a mechanism similar to that proposed in project I. Here, we hypothesized that, HPV-infected cells transport exosomes containing oxidative stress factors, especially CYPs to monocytes/macrophages latently infected with HIV-1. The CYPs from the exosomes are released into the infected macrophages and monocytes where they add to the effect of the cellular CYPs to mediate oxidative stress and ultimately HIV-1 replication.

As expected, our results indicated that, out of the five compounds tested, chronic exposure (7 days) of BaP significantly increased oxidative stress and cytotoxicity in U937 monocytic cells and human primary monocytic cells. Acute exposure of BaP did not cause any significant increase in oxidative stress and cytotoxicity in U937 cells, probably because of the protective effect from the basal antioxidant enzymes. Chronic exposure of $\mathrm{BaP}$ also induced the mRNA expression of CYPs (CYP1A1 and CYP3A4) and AOEs (SOD1 and catalase) in the monocytic cells, which indicated the possible involvement of CYPs and oxidative stress pathway in BaP-induced cytotoxicity. Further, 
the increased caspase- 3 activity after chronic $\mathrm{BaP}$ exposure suggested that $\mathrm{BaP}$ may be causing cytotoxicity possibly via a caspase-3-dependent apoptotic pathway. Therefore, we demonstrated that chronic treatment of $\mathrm{BaP}$ induces cytotoxicity in monocytes, possibly through generation of ROS via a CYP pathway.

We also demonstrated that BaP induced oxidative stress and HIV-1 replication in U1 cells as well as HIV-1-infected human primary macrophages. Treatment of antioxidants and CYP1A1 inhibitor significantly decreased the oxidative stress and viral replication induced by $\mathrm{BaP}$, indicating that $\mathrm{BaP}$-induced $\mathrm{HIV}-1$ replication occurs via a CYP-mediated oxidative stress pathway. Further, we also showed that ROS generated via CYP metabolism of $\mathrm{BaP}$ facilitates the nuclear translocation of NF- $\kappa \mathrm{B}$ subunits, leading to enhanced HIV-1 transcription. We also confirmed the role of NF- $\kappa$ B pathway using specific NF- $\kappa \mathrm{B}$ inhibitors. Our results give an insight of a novel mechanism for smokinginduced HIV-1 replication, that CYP-mediated metabolism of $\mathrm{BaP}$ generates ROS, which triggers $\mathrm{NF}-\kappa \mathrm{B}$ pathway to enhance the transcription of HIV-1 genome.

Next, we observed that, exosomes derived from HPV-infected caski cells package oxidative stress factors (like CYPs, HPV oncoprotein and antioxidant enzymes) and diffuse into the U1 cells. We also observed that the treatment with these exosomes enhance oxidative stress and HIV-1 replication in U1 cells. Antioxidants and specific CYP inhibitors significantly decreased exosomes-induced HIV-1 replication in U1 cells. This confirms, at least in part, the role of oxidative stress and CYPs in the exosomemediated HIV-1 replication. Our results demonstrate that HPV-infected cells induce HIV-1 replication in monocytic cells latently infected with HIV-1 via exosomes containing CYPs. These CYPs along with the cellular CYPs may be generating ROS during the metabolism of various endogenous and exogenous substrates within the cells, thereby resulting oxidative stress and ultimately HIV-1 replication.

Overall, our study illustrates that comorbidities like smoking or HPV coinfection contribute to HIV-1 replication and pathogenesis in monocytic cells, at least in part, via a CYP-mediated oxidative stress pathway. This study is clinically relevant as it provides the basis for potentially novel therapeutic targets like CYPs and oxidative stress. Since there are no guidelines to titrate ART regimens in smokers, the outcomes from the present study will also help in optimizing ART regimens for HIV-1-positive smokers. Identification of the role of CYP enzymes in tobacco/HPV-mediated HIV-1 pathogenesis would not only help to adjust the ART regimen, but would also help to design a better treatment strategy for HIV-1-positive patients with tobacco addiction or HPVcoinfection. CYP enzymes are an important target for drug development to combat many disease conditions, such as cancer (CYP1A1, 1A2, 1B1, 2A13) [369, 370], cardiovascular disease (CYP1A, 1A2, 1B1) [371], inflammation and pain (CYP4A) [372], hypertension [373], pulmonary disease (CYP1B1) [374], and HIV-1 (CYP3A4) [153]. In the present study, we demonstrated that antioxidants (resveratrol, vitamin $\mathrm{E}$ and C) and CYP inhibitors (ellipticine and tryptamine) reduce HIV-1 viral load in vitro in $\mathrm{BaP}$ or HPV-exposed U1 cells. These compounds can further be optimized and formulated into potent and clinically safe drug molecules to alleviate disease progression in PLWHA who are either smokers or have HPV coinfection. Furthermore, we have 
shown that chemo-dietary agents such as curcumin and cucurbitacin-D reduce HPVinduced HIV-1 replication in monocytic cells. Curcumin and cucurbitacin-D have been shown to have a significant impact on alleviating cervical cancer [344]. The identification of these compounds provides a novel insight for the design and development of new drugs, or their use as adjuvant therapy to alleviate both HIV-1 and HPV infection in PLWHA.

Moreover, these antioxidants, CYP inhibitors, and chemo-dietary agents can be synthetically loaded into exosomes and delivered to the target tissues. Exosomes are nonimmunogenic and biocompatible; they also have target specificity, a long circulating halflife, and no associated toxicological problems. These features make exosomes a promising drug delivery system, compared to synthetically constructed nano-carriers [375]. Recently, exosomes derived from brain cells have been shown to be an effective drug carrier to deliver anticancer drugs across the BBB for the treatment of brain cancer in a zebrafish model [376]. In another study, dendritic cells were engineered to produce exosomes that expressed exosomal membrane proteins fused to the neuron-specific RVG peptide. The RVG-targeted exosomes were capable of delivering siRNA targeted to BACE1, a therapeutic target of Alzheimer's disease, to brain tissues of mice, resulting in successful BACE1 knockdown [377]. Furthermore, exosomes loaded with antioxidant agents such as curcumin have also been reported to have a relatively improved distribution in brain tissues and reduce loss of neurons, glia, and brain endothelia after ischemia-reperfusion injury [378]. The exosomes loaded with antioxidants or CYP inhibitors or chemo-dietary agents can also be therapeutically beneficial to reduce the oxidative stress-induced HIV-1 replication in monocytic cells of HIV-1-infected smokers as well as HIV-1 patients with HPV co-infection.

HIV-1-infected monocytes and macrophages circulating in the bloodstream can spread HIV-1 infection in the CNS once they cross the BBB [379]. These cells further activate astrocytes and microglia to produce various inflammatory chemokines and cytokines. The release of cytokines, chemokines, viral particles, and ROS causes oxidative stress and inflammation within the brain, ultimately leading to HIV-1associated neurological disorders (HAND) [380]. Currently available ART drugs have very low bioavailability at the brain tissues as their diffusion to the CNS is limited by BBB [381]. The viral reservoirs and the infected cells within the brain are therefore protected from ART, which further makes it difficult to attain the complete eradication of HIV-1. As exosomes can easily cross the BBB, the delivery of antioxidant/ART-loaded exosomes can be an efficient therapeutic strategy to reduce the viral load in brain cells by lowering ROS level. The use of exosomes as a therapeutic carrier to deliver ART in the brain can also help to reduce HAND, which has been a rising problem among PLWHA in the ART era.

In addition to smoking, individuals infected with HIV-1 often use various other drugs of abuse such as cocaine, methamphetamine, marijuana, and alcohol, which result in similar oxidative damage. The concomitant use of these drugs is likely to further exacerbate oxidative stress and viral load in PLWHA. Thus our strategy discussed above that contain CYP inhibitors/antioxidants/chemo-dietary agents loaded-exosomes may 
serve to alleviate oxidative damage due to abuse of these poly-drugs in different cells and tissues. Furthermore, these exosomes may also have therapeutic benefits in other neuropathological conditions associated with oxidative stress such as Alzheimer's disease, Parkinson's disease, and multiple sclerosis.

The International Agency for research on Cancer (IARC) has classified smoking as one of the risk factors for cervical cancer [212]. Several studies indicated that cigarette smoking enhances the progression of HPV infection into cervical lesions and cancer [381]. A meta-analysis study conducted by the International Collaboration of Epidemiological Studies of Cervical Cancer on 23 different studies concluded that the relative risk is 1.6 times higher for a current smoker to develop HPV infection into squamous cell carcinoma, compared to a non-smoker [382]. Cigarette smoke constituents such as nicotine and benzo(a)pyrene are found to be present in the mucus of cervical cancer patients [204]. Tobacco has also been shown to enhance the transcription of HPV oncoproteins E6 and E7 that reduce p53 levels and activity, enhance DNA damage and mutation, thereby leading to cervical cancer [383]. Nicotine, $\mathrm{BaP}$, and their metabolites are known to form DNA and protein adducts that induce carcinogenesis in cervix [384]. Moreover, BaP has also been shown to increase HPV synthesis in cervical cancer cells [385]. In the present study, we have shown how smoking and HPV independently impact HIV-1 replication in monocytic cells. As smoking enhances HPV pathogenesis and progression to cervical cancer, these factors together may further have an additive or synergistic effect on the HIV-1 replication in monocytic cells. Therefore, in future, our lab will be investigating the combined effect of smoking and HPV coinfection on HIV-1 replication in monocytic cells.

Numerous CYPs are upregulated in cervical cancer cells including the CYPs that metabolize cigarette smoke constituents, especially polyaryl hydrocarbons (CYP1A1/1A2/1B1) [386]. Exposure of polyaryl hydrocarbons, especially BaP, may enhance oxidative stress in cervical cancer cells via CYP pathway. The packaging of oxidative stress factors into the exosomes, as well as the release of the exosomes may be altered after $\mathrm{BaP}$ exposure into the cervical cancer cells. In the current project, we have only demonstrated the role of CYPs in the exosomes. We will be investigating other oxidative stress factors in the exosomes that are responsible for reactivating latent HIV-1 in monocytic cells, using proteomic and microarray analysis. Based on their known contribution in reactivating latent HIV-1, we speculate that cytokines (IL-6, TNF- $\alpha$ ) and miRNAs $(34 a, 181)$ may have a role in activating HIV-1 replication in monocytic cells. We also observed the presence of HPV oncoprotein E6 in the exosomes derived from HPV-infected cervical cancer cells, which adds the possibility that these oncoproteins may also have a role in generating oxidative stress in the recipient cells. We propose that the exosomes derived from cervical cancer cells, exposed to $\mathrm{BaP}$ may have a greater potential to reactivate latent HIV-1 in monocytic cells than the exosomes from cervical cancer cells alone. Overall, this new project will demonstrate how smoking and HPV coinfection together impact reactivation of latent HIV-1 in viral reservoirs such as monocytes. 


\section{LIST OF REFERENCES}

1. AVERT. Global information and education on HIV and AIDS. 2018; Available from: https://www.avert.org/global-hiv-and-aids-statistics\#footnote1 kgohmwz.

2. $\quad$ Prevention, C.f.D.C.a. HIV in the United States: At A Glance. 2018; Available from: https://www.cdc.gov/hiv/statistics/overview/ataglance.html.

3. $\quad$ Robbins, H.A., et al., Excess cancers among HIV-infected people in the United States. J Natl Cancer Inst, 2015. 107(4).

4. Kahn, J.A., et al., Prevalence and risk factors for oral DNA tumor viruses in HIVinfected youth. J Med Virol, 2016. 88(11): p. 1944-52.

5. Sacktor, N., et al., Prevalence of HIV-associated neurocognitive disorders in the Multicenter AIDS Cohort Study. Neurology, 2016. 86(4): p. 334-40.

6. German Advisory Committee Blood, S.A.o.P.T.b.B., Human Immunodeficiency Virus (HIV). Transfus Med Hemother, 2016. 43(3): p. 203-22.

7. Chan, D.C. and P.S. Kim, HIV entry and its inhibition. Cell, 1998. 93(5): p. 6814.

8. Kwon, D.S., et al., DC-SIGN-mediated internalization of HIV is required for trans-enhancement of T cell infection. Immunity, 2002. 16(1): p. 135-44.

9. Gobeil, L.A., R. Lodge, and M.J. Tremblay, Differential HIV-1 endocytosis and susceptibility to virus infection in human macrophages correlate with cell activation status. J Virol, 2012. 86(19): p. 10399-407.

10. Le Grice, S.F., Human immunodeficiency virus reverse transcriptase: 25 years of research, drug discovery, and promise. J Biol Chem, 2012. 287(49): p. 40850-7.

11. McDonald, D., et al., Visualization of the intracellular behavior of HIV in living cells. J Cell Biol, 2002. 159(3): p. 441-52.

12. Rohdewohld, H., et al., Retrovirus integration and chromatin structure: Moloney murine leukemia proviral integration sites map near DNase I-hypersensitive sites. J Virol, 1987. 61(2): p. 336-43.

13. Greenway, A.L., et al., Human immunodeficiency virus type 1 Nef binds to tumor suppressor 553 and protects cells against p53-mediated apoptosis. J Virol, 2002. 76(6): p. 2692-702.

14. Zheng, Y.H., et al., Nef increases infectivity of HIV via lipid rafts. Curr Biol, 2001. 11(11): p. 875-9.

15. Goncalves, J. and M. Santa-Marta, HIV-1 Vif and APOBEC3G: multiple roads to one goal. Retrovirology, 2004. 1: p. 28.

16. Garrus, J.E., et al., Tsg101 and the vacuolar protein sorting pathway are essential for HIV-1 budding. Cell, 2001. 107(1): p. 55-65.

17. Wilk, T., et al., Organization of immature human immunodeficiency virus type 1. J Virol, 2001. 75(2): p. 759-71.

18. Swanstrom, R. and J.W. Wills, Synthesis, Assembly, and Processing of Viral Proteins, in Retroviruses, J.M. Coffin, S.H. Hughes, and H.E. Varmus, Editors. 1997: Cold Spring Harbor (NY).

19. Kumar, A., W. Abbas, and G. Herbein, HIV-1 latency in monocytes/macrophages. Viruses, 2014. 6(4): p. 1837-60. 
20. Kelly, J., et al., Human macrophages support persistent transcription from unintegrated HIV-1 DNA. Virology, 2008. 372(2): p. 300-12.

21. Wang, X., et al., Cellular microRNA expression correlates with susceptibility of monocytes/macrophages to HIV-1 infection. Blood, 2009. 113(3): p. 671-4.

22. Deeks, S.G., et al., HIV infection. Nature Reviews Disease Primers, 2015. 1: p. 15035.

23. Services, D.o.H.a.H. Guidelines for the use of antiretrovirals agents in adults and adolescents living with HIV. 2018; Available from: https://aidsinfo.nih.gov/contentfiles/lvguidelines/adultandadolescentgl.pdf.

24. Services, U.D.o.H.a.H. HIV Treatment. 2018; Available from: https://aidsinfo.nih.gov/understanding-hiv-aids/fact-sheets/21/58/fda-approvedhiv-medicines.

25. Arts, E.J. and D.J. Hazuda, HIV-1 antiretroviral drug therapy. Cold Spring Harb Perspect Med, 2012. 2(4): p. a007161.

26. Briz, V., E. Poveda, and V. Soriano, HIV entry inhibitors: mechanisms of action and resistance pathways. J Antimicrob Chemother, 2006. 57(4): p. 619-27.

27. Bruno, C.J. and J.M. Jacobson, Ibalizumab: an anti-CD4 monoclonal antibody for the treatment of HIV-1 infection. J Antimicrob Chemother, 2010. 65(9): p. 183941.

28. Xu, L., et al., Cobicistat (GS-9350): A Potent and Selective Inhibitor of Human CYP3A as a Novel Pharmacoenhancer. ACS Med Chem Lett, 2010. 1(5): p. 20913.

29. Kumar, S., et al., Alcohol consumption effect on antiretroviral therapy and HIV-1 pathogenesis: role of cytochrome P450 isozymes. Expert Opin Drug Metab Toxicol, 2012. 8(11): p. 1363-75.

30. Abuse, N.I.o.D. Drug and Alcohol Use - A Significant Risk Factor for HIV. 2018; Available from: https://www.drugabuse.gov/related-topics/trendsstatistics/infographics/drug-alcohol-use-significant-risk-factor-hiv.

31. Rosen, M.I., et al., Association between use of specific drugs and antiretroviral adherence: findings from MACH 14. AIDS Behav, 2013. 17(1): p. 142-7.

32. Gamarel, K.E., et al., Prevalence and correlates of substance use among youth living with HIV in clinical settings. Drug Alcohol Depend, 2016. 169: p. 11-18.

33. Kapadia, F., et al., The role of substance abuse in HIV disease progression: reconciling differences from laboratory and epidemiologic investigations. Clin Infect Dis, 2005. 41(7): p. 1027-34.

34. Kumar, S., et al., Drug-drug interactions between anti-retroviral therapies and drugs of abuse in HIV systems. Expert Opin Drug Metab Toxicol, 2015. 11(3): p. 343-55.

35. Tucker, J.S., et al., Substance use and mental health correlates of nonadherence to antiretroviral medications in a sample of patients with human immunodeficiency virus infection. Am J Med, 2003. 114(7): p. 573-80.

36. Mdodo, R., et al., Cigarette smoking prevalence among adults with HIV compared with the general adult population in the United States: cross-sectional surveys. Ann Intern Med, 2015. 162(5): p. 335-44. 
37. Shirley, D.K., R.J. Kaner, and M.J. Glesby, Effects of smoking on non-AIDSrelated morbidity in HIV-infected patients. Clin Infect Dis, 2013. 57(2): p. 27582.

38. Rahmanian, S., et al., Cigarette smoking in the HIV-infected population. Proc Am Thorac Soc, 2011. 8(3): p. 313-9.

39. Ockene, I.S. and N.H. Miller, Cigarette smoking, cardiovascular disease, and stroke: a statement for healthcare professionals from the American Heart Association. American Heart Association Task Force on Risk Reduction. Circulation, 1997. 96(9): p. 3243-7.

40. Lortet-Tieulent, J., et al., Cigarette smoking-attributable burden of cancer by race and ethnicity in the United States. Cancer Causes Control, 2017. 28(9): p. 981984.

41. Helleberg, M., et al., Mortality attributable to smoking among HIV-1-infected individuals: a nationwide, population-based cohort study. Clin Infect Dis, 2013. 56(5): p. 727-34.

42. da Silva, C.M., et al., Alcohol use disorders among people living with HIV/AIDS in Southern Brazil: prevalence, risk factors and biological markers outcomes. BMC Infect Dis, 2017. 17(1): p. 263.

43. Galvan, F.H., et al., The prevalence of alcohol consumption and heavy drinking among people with HIV in the United States: results from the HIV Cost and Services Utilization Study. J Stud Alcohol, 2002. 63(2): p. 179-86.

44. Chander, G., B. Lau, and R.D. Moore, Hazardous alcohol use: a risk factor for non-adherence and lack of suppression in HIV infection. J Acquir Immune Defic Syndr, 2006. 43(4): p. 411-7.

45. Chander, G., Addressing alcohol use in HIV-infected persons. Top Antivir Med, 2011. 19(4): p. 143-7.

46. Suwa G. Goar, M.D.A., Michael T. Agbir \& Edward Dochalson, Prevalence and socio-demographic correlates of alcohol use disorders among HIV patients African Journal of Drug \& Alcohol Studies. 2011.

47. Cook, R.L., et al., Alcohol consumption patterns and HIV viral suppression among persons receiving HIV care in Florida: an observational study. Addict Sci Clin Pract, 2017. 12(1): p. 22.

48. Haorah, J., et al., Mechanism of alcohol-induced oxidative stress and neuronal injury. Free Radic Biol Med, 2008. 45(11): p. 1542-50.

49. Hoek, J.B. and J.G. Pastorino, Ethanol, oxidative stress, and cytokine-induced liver cell injury. Alcohol, 2002. 27(1): p. 63-8.

50. Jin, M., et al., Regulation of cytochrome P450 2el expression by ethanol: role of oxidative stress-mediated pkc/jnk/sp1 pathway. Cell Death Dis, 2013. 4: p. e554.

51. Smothers, C.T., J.J. Mrotek, and D.M. Lovinger, Chronic ethanol exposure leads to a selective enhancement of $N$-methyl-D-aspartate receptor function in cultured hippocampal neurons. J Pharmacol Exp Ther, 1997. 283(3): p. 1214-22.

52. Brailoiu, E., et al., Acute exposure to ethanol potentiates human immunodeficiency virus type 1 Tat-induced $\mathrm{Ca}(2+)$ overload and neuronal death in cultured rat cortical neurons. J Neurovirol, 2006. 12(1): p. 17-24. 
53. Acheampong, E., et al., Ethanol strongly potentiates apoptosis induced by HIV-1 proteins in primary human brain microvascular endothelial cells. Virology, 2002. 304(2): p. 222-34.

54. Green, J.E., R.V. Saveanu, and R.A. Bornstein, The effect of previous alcohol abuse on cognitive function in HIV infection. Am J Psychiatry, 2004. 161(2): p. 249-54.

55. Persidsky, Y., et al., HIV-1 infection and alcohol abuse: neurocognitive impairment, mechanisms of neurodegeneration and therapeutic interventions. Brain Behav Immun, 2011. 25 Suppl 1: p. S61-70.

56. Crane, H.M., et al., Prevalence and Factors Associated with Hazardous Alcohol Use Among Persons Living with HIV Across the US in the Current Era of Antiretroviral Treatment. AIDS Behav, 2017. 21(7): p. 1914-1925.

57. Okafor, C.N., et al., Marijuana use and viral suppression in persons receiving medical care for HIV-infection. Am J Drug Alcohol Abuse, 2017. 43(1): p. 103110.

58. de Jong, B.C., et al., Marijuana use and its association with adherence to antiretroviral therapy among HIV-infected persons with moderate to severe nausea. J Acquir Immune Defic Syndr, 2005. 38(1): p. 43-6.

59. Mimiaga, M.J., et al., Substance use among HIV-infected patients engaged in primary care in the United States: findings from the Centers for AIDS Research Network of Integrated Clinical Systems cohort. Am J Public Health, 2013. 103(8): p. 1457-67.

60. Fogarty, A., et al., Marijuana as therapy for people living with HIV/AIDS: social and health aspects. AIDS Care, 2007. 19(2): p. 295-301.

61. Bonn-Miller, M.O., et al., Cannabis use and HIV antiretroviral therapy adherence and HIV-related symptoms. J Behav Med, 2014. 37(1): p. 1-10.

62. Wilson, K.J., A. Doxanakis, and C.K. Fairley, Predictors for non-adherence to antiretroviral therapy. Sex Health, 2004. 1(4): p. 251-7.

63. Corless, I.B., et al., Marijuana effectiveness as an HIV self-care strategy. Clin Nurs Res, 2009. 18(2): p. 172-93.

64. Slawson, G., et al., High-intensity cannabis use and adherence to antiretroviral therapy among people who use illicit drugs in a Canadian setting. AIDS Behav, 2015. 19(1): p. 120-7.

65. Allshouse, A.A., et al., The Impact of Marijuana Use on the Successful Aging of HIV-Infected Adults. J Acquir Immune Defic Syndr, 2015. 69(2): p. 187-92.

66. Thames, A.D., et al., Combined effects of HIV and marijuana use on neurocognitive functioning and immune status. AIDS Care, 2016. 28(5): p. 62832.

67. Chang, L., et al., Combined and independent effects of chronic marijuana use and HIV on brain metabolites. J Neuroimmune Pharmacol, 2006. 1(1): p. 65-76.

68. Skalski, L.M., et al., The Impact of Marijuana Use on Memory in HIV-Infected Patients: A Comprehensive Review of the HIV and Marijuana Literatures. Curr Drug Abuse Rev, 2016. 9(2): p. 126-141.

69. Skalski, L.M., et al., Memory Impairment in HIV-Infected Individuals with Early and Late Initiation of Regular Marijuana Use. AIDS Behav, 2018. 22(5): p. 15961605. 
70. Morgan, E., et al., Marijuana Use as a Sex-Drug is Associated with HIV Risk Among Black MSM and Their Network. AIDS Behav, 2016. 20(3): p. 600-7.

71. Abrams, D.I., et al., Short-term effects of cannabinoids in patients with HIV-1 infection: a randomized, placebo-controlled clinical trial. Ann Intern Med, 2003. 139(4): p. 258-66.

72. Molina, P.E., et al., Cannabinoid administration attenuates the progression of simian immunodeficiency virus. AIDS Res Hum Retroviruses, 2011. 27(6): p. 585-92.

73. Amedee, A.M., et al., Chronic Delta(9)-tetrahydrocannabinol administration may not attenuate simian immunodeficiency virus disease progression in female rhesus macaques. AIDS Res Hum Retroviruses, 2014. 30(12): p. 1216-25.

74. Jacquet, J.M., et al., Psychoactive substances, alcohol and tobacco consumption in HIV-infected outpatients. AIDS, 2018. 32(9): p. 1165-1171.

75. Grabovac, I., et al., Prevalence and Associations of Illicit Drug and Polydrug Use in People Living with HIV in Vienna. Scientific Reports, 2018. 8(1): p. 8046.

76. Arnsten, J.H., et al., Impact of active drug use on antiretroviral therapy adherence and viral suppression in HIV-infected drug users. J Gen Intern Med, 2002. 17(5): p. 377-81.

77. Lucas, G.M., et al., Detrimental effects of continued illicit drug use on the treatment of HIV-1 infection. J Acquir Immune Defic Syndr, 2001. 27(3): p. 2519.

78. Baum, M.K., et al., Crack-cocaine use accelerates HIV disease progression in a cohort of HIV-positive drug users. J Acquir Immune Defic Syndr, 2009. 50(1): p. 93-9.

79. Nair, M.P., et al., Effect of cocaine on chemokine and CCR-5 gene expression by mononuclear cells from normal donors and HIV-1 infected patients. Adv Exp Med Biol, 2001. 493: p. 235-40.

80. Nair, M.P., et al., Cocaine differentially modulates chemokine production by mononuclear cells from normal donors and human immunodeficiency virus type 1-infected patients. Clin Diagn Lab Immunol, 2000. 7(1): p. 96-100.

81. Addai, A.B., et al., Cocaine modulates HIV-1 integration in primary CD4+ $T$ cells: implications in HIV-1 pathogenesis in drug-abusing patients. J Leukoc Biol, 2015. 97(4): p. 779-90.

82. Napuri, J., et al., Cocaine enhances HIV-1 infectivity in monocyte derived dendritic cells by suppressing microRNA-155. PLoS One, 2013. 8(12): p. e83682.

83. Pandhare, J., et al., Cocaine enhances HIV-1-induced CD4(+) T-cell apoptosis: implications in disease progression in cocaine-abusing HIV-1 patients. Am J Pathol, 2014. 184(4): p. 927-936.

84. Buch, S., et al., Cocaine and HIV-1 interplay in CNS: cellular and molecular mechanisms. Curr HIV Res, 2012. 10(5): p. 425-8.

85. Reynolds, J.L., et al., Proteomic analysis of the effects of cocaine on the enhancement of HIV-1 replication in normal human astrocytes (NHA). Brain Res, 2006. 1123(1): p. 226-36.

86. Yao, H., et al., Cocaine and human immunodeficiency virus type 1 gpl20 mediate neurotoxicity through overlapping signaling pathways. J Neurovirol, 2009. 15(2): p. 164-75. 
87. Dahal, S., et al., Interactive effects of cocaine on HIV infection: implication in HIV-associated neurocognitive disorder and neuroAIDS. Front Microbiol, 2015. 6: p. 931.

88. Health, N.I.o. Address HIV-Associated Comorbidities, Coinfections, and Complications. 2018; Available from: https://www.oar.nih.gov/hiv-policy-andresearch/research-priorities-overview/address-hiv-associated-comorbiditiescoinfections-complications.

89. Organizations, W.H. Tuberculosis and HIV. 2018; Available from: http://www.who.int/hiv/topics/tb/about tb/en/.

90. Organizations, W.H. TB/HIV facts 2012-2013. 2018; Available from: http://www.who.int/hiv/topics/tb/tbhiv facts 2013/en/.

91. Winter, J.R.A., A. L.; Gupta, R. K.; Stagg, H. R.; Delpech, V.; Abubakar, I., Tuberculosis infection and disease in people living with HIV in countries with low tuberculosis incidence. International Union Against Tuberculosis and Lung Disease, 2018. 22(713-722).

92. Lewinsohn, D.A., et al., Mycobacterium tuberculosis-specific CD8+T cells preferentially recognize heavily infected cells. Am J Respir Crit Care Med, 2003. 168(11): p. 1346-52.

93. Patel, N.R., et al., HIV impairs TNF-alpha mediated macrophage apoptotic response to Mycobacterium tuberculosis. J Immunol, 2007. 179(10): p. 6973-80.

94. Kumawat, K., et al., Exogenous Nef is an inhibitor of Mycobacterium tuberculosis-induced tumor necrosis factor-alpha production and macrophage apoptosis. J Biol Chem, 2010. 285(17): p. 12629-37.

95. Lawn, S.D., et al., Anatomically compartmentalized human immunodeficiency virus replication in HLA-DR+ cells and CD14+ macrophages at the site of pleural tuberculosis coinfection. J Infect Dis, 2001. 184(9): p. 1127-33.

96. Zhang, Y., et al., Mycobacterium tuberculosis enhances human immunodeficiency virus-1 replication by transcriptional activation at the long terminal repeat. J Clin Invest, 1995. 95(5): p. 2324-31.

97. Hoshino, Y., et al., Maximal HIV-1 replication in alveolar macrophages during tuberculosis requires both lymphocyte contact and cytokines. J Exp Med, 2002. 195(4): p. 495-505.

98. Ranjbar, S., et al., Regulation of Mycobacterium tuberculosis-dependent HIV-1 transcription reveals a new role for NFAT5 in the toll-like receptor pathway. PLoS Pathog, 2012. 8(4): p. e1002620.

99. Honda, Y., et al., Type I interferon induces inhibitory 16-kD CCAAT/ enhancer binding protein (C/EBP)beta, repressing the HIV-1 long terminal repeat in macrophages: pulmonary tuberculosis alters C/EBP expression, enhancing HIV-1 replication. J Exp Med, 1998. 188(7): p. 1255-65.

100. Domingo-Gonzalez, R., et al., Cytokines and Chemokines in Mycobacterium tuberculosis Infection. Microbiol Spectr, 2016. 4(5).

101. Bell, L.C.K. and M. Noursadeghi, Pathogenesis of HIV-1 and Mycobacterium tuberculosis co-infection. Nature Reviews Microbiology, 2017. 16: p. 80.

102. Alter, M.J., Epidemiology of viral hepatitis and HIV co-infection. J Hepatol, 2006. 44(1 Suppl): p. S6-9. 
103. Prevention, C.f.D.C.a. HIV and Viral Hepatitis. 2014; Available from: https://www.cdc.gov/hiv/pdf/library factsheets hiv and viral hepatitis.pdf.

104. Kim, A.Y., et al., The magnitude and breadth of hepatitis $C$ virus-specific CD8+ $T$ cells depend on absolute CD4+T-cell count in individuals coinfected with HIV1. Blood, 2005. 105(3): p. 1170-8.

105. Harcourt, G., et al., Diminished frequency of hepatitis $C$ virus specific interferon gamma secreting CD4+ T cells in human immunodeficiency virus/hepatitis $C$ virus coinfected patients. Gut, 2006. 55(10): p. 1484-7.

106. Lin, W., et al., HIV increases HCV replication in a TGF-betal-dependent manner. Gastroenterology, 2008. 134(3): p. 803-11.

107. Wright, T.L., et al., Hepatitis $C$ in HIV-infected patients with and without AIDS: prevalence and relationship to patient survival. Hepatology, 1994. 20(5): p. 11525.

108. Macias, J., et al., Influence of hepatitis $C$ virus infection on the mortality of antiretroviral-treated patients with HIV disease. Eur J Clin Microbiol Infect Dis, 1998. 17(3): p. 167-70.

109. Dorrucci, M., et al., Coinfection of hepatitis $C$ virus with human immunodeficiency virus and progression to AIDS. Italian Seroconversion Study. J Infect Dis, 1995. 172(6): p. 1503-8.

110. Sulkowski, M.S., et al., Hepatitis $C$ and progression of HIV disease. JAMA, 2002. 288(2): p. 199-206.

111. Konopnicki, D., et al., Hepatitis B and HIV: prevalence, AIDS progression, response to highly active antiretroviral therapy and increased mortality in the EuroSIDA cohort. AIDS, 2005. 19(6): p. 593-601.

112. Greub, G., et al., Clinical progression, survival, and immune recovery during antiretroviral therapy in patients with HIV-1 and hepatitis $C$ virus coinfection: the Swiss HIV Cohort Study. Lancet, 2000. 356(9244): p. 1800-5.

113. Klein, M.B., R.G. Lalonde, and S. Suissa, The impact of hepatitis $C$ virus coinfection on HIV progression before and after highly active antiretroviral therapy. J Acquir Immune Defic Syndr, 2003. 33(3): p. 365-72.

114. Wit, F.W.N.M., et al., Incidence of and Risk Factors for Severe Hepatotoxicity Associated with Antiretroviral Combination Therapy. The Journal of Infectious Diseases, 2002. 186(1): p. 23-31.

115. Qadri, I., H.F. Maguire, and A. Siddiqui, Hepatitis B virus transactivator protein X interacts with the TATA-binding protein. Proc Natl Acad Sci U S A, 1995. 92(4): p. 1003-7.

116. Ng'andwe, C., et al., The distribution of sexually-transmitted Human Papillomaviruses in HIV positive and negative patients in Zambia, Africa. BMC Infect Dis, 2007. 7: p. 77.

117. Whitham, H.K., et al., A Comparison of the Natural History of HPV Infection and Cervical Abnormalities among HIV-Positive and HIV-Negative Women in Senegal, Africa. Cancer Epidemiol Biomarkers Prev, 2017. 26(6): p. 886-894.

118. Petry, K.U., et al., Cellular immunodeficiency enhances the progression of human papillomavirus-associated cervical lesions. Int J Cancer, 1994. 57(6): p. 836-40. 
119. Abraham, A.G., et al., Invasive cervical cancer risk among HIV-infected women: a North American multicohort collaboration prospective study. J Acquir Immune Defic Syndr, 2013. 62(4): p. 405-13.

120. Heard, I., et al., Increased risk of cervical disease among human immunodeficiency virus-infected women with severe immunosuppression and high human papillomavirus load(1). Obstet Gynecol, 2000. 96(3): p. 403-9.

121. Tugizov, S.M., et al., HIV-associated disruption of mucosal epithelium facilitates paracellular penetration by human papillomavirus. Virology, 2013. 446(1-2): p. 378-88.

122. Theiler, R.N., et al., High-risk human papillomavirus reactivation in human immunodeficiency virus-infected women: risk factors for cervical viral shedding. Obstet Gynecol, 2010. 115(6): p. 1150-8.

123. Vernon, S.D., et al., The HIV-1 tat protein enhances E2-dependent human papillomavirus 16 transcription. Virus Res, 1993. 27(2): p. 133-45.

124. Houlihan, C.F., et al., Human papillomavirus infection and increased risk of HIV acquisition. A systematic review and meta-analysis. AIDS, 2012. 26(17): p. 221122.

125. Low, A.J., et al., Genital warts and infection with human immunodeficiency virus in high-risk women in Burkina Faso: a longitudinal study. BMC Infect Dis, 2011. 11: p. 20 .

126. Averbach, S.H., et al., The association between cervical human papillomavirus infection and HIV acquisition among women in Zimbabwe. AIDS, 2010. 24(7): p. 1035-42.

127. Smith-McCune, K.K., et al., Type-specific cervico-vaginal human papillomavirus infection increases risk of HIV acquisition independent of other sexually transmitted infections. PLoS One, 2010. 5(4): p. e10094.

128. Laurson, J., et al., Epigenetic repression of E-cadherin by human papillomavirus 16 E7 protein. Carcinogenesis, 2010. 31(5): p. 918-26.

129. Williamson, A.L., The Interaction between Human Immunodeficiency Virus and Human Papillomaviruses in Heterosexuals in Africa. J Clin Med, 2015. 4(4): p. 579-92.

130. Kemp, T.J., et al., Elevated systemic levels of inflammatory cytokines in older women with persistent cervical human papillomavirus infection. Cancer Epidemiol Biomarkers Prev, 2010. 19(8): p. 1954-9.

131. Spear, G.T., et al., Positive association between HIV RNA and IL-6 in the genital tract of Rwandan women. AIDS Res Hum Retroviruses, 2008. 24(7): p. 973-6.

132. Lane, B.R., et al., Interleukin-8 stimulates human immunodeficiency virus type 1 replication and is a potential new target for antiretroviral therapy. J Virol, 2001. 75(17): p. 8195-202.

133. Chun, T.W., et al., Induction of HIV-1 replication in latently infected CD4+T cells using a combination of cytokines. J Exp Med, 1998. 188(1): p. 83-91.

134. Marullo, R., et al., HPV16 E6 and E7 proteins induce a chronic oxidative stress response via NOX2 that causes genomic instability and increased susceptibility to DNA damage in head and neck cancer cells. Carcinogenesis, 2015. 36(11): p. 1397-406. 
135. Hile, S.J., et al., Recent Tobacco Smoking is Associated with Poor HIV Medical Outcomes Among HIV-Infected Individuals in New York. AIDS Behav, 2016. 20(8): p. 1722-9.

136. Bekele, T., et al., Trends and Correlates of Cigarette Smoking and Its Impacts on Health-Related Quality of Life Among People Living with HIV: Findings from the Ontario HIV Treatment Network Cohort Study, 2008-2014. AIDS Patient Care STDS, 2017. 31(2): p. 49-59.

137. Akhtar-Khaleel, W.Z., et al., Long-Term Cigarette Smoking Trajectories Among HIV-Seropositive and Seronegative MSM in the Multicenter AIDS Cohort Study. AIDS Behav, 2016. 20(8): p. 1713-21.

138. Akhtar-Khaleel, W.Z., et al., Trends and Predictors of Cigarette Smoking Among HIV Seropositive and Seronegative Men: The Multicenter Aids Cohort Study. AIDS Behav, 2016. 20(3): p. 622-32.

139. Hirshfield, S., et al., Drug Use, Sexual Risk, and Syndemic Production Among Men Who Have Sex With Men Who Engage in Group Sexual Encounters. Am J Public Health, 2015. 105(9): p. 1849-58.

140. Marshall, M.M., M.C. McCormack, and G.D. Kirk, Effect of cigarette smoking on HIV acquisition, progression, and mortality. AIDS Educ Prev, 2009. 21(3 Suppl): p. 28-39.

141. Jones, S.L. and G.F. Gebhart, Characterization of coeruleospinal inhibition of the nociceptive tail-flick reflex in the rat: mediation by spinal alpha 2-adrenoceptors. Brain Res, 1986. 364(2): p. 315-30.

142. Pacek, L.R., et al., Current cigarette smoking among HIV-positive current and former drug users: associations with individual and social characteristics. AIDS Behav, 2014. 18(7): p. 1368-77.

143. Brath, H., et al., Prevalence and Correlates of Smoking and Readiness to Quit Smoking in People Living with HIV in Austria and Germany. PLoS One, 2016. 11(2): p. e0150553.

144. Pollack, T.M., et al., Cigarette smoking is associated with high HIV viral load among adults presenting for antiretroviral therapy in Vietnam. PLoS One, 2017. 12(3): p. e0173534.

145. Mazzone, P., et al., Pathophysiological impact of cigarette smoke exposure on the cerebrovascular system with a focus on the blood-brain barrier: expanding the awareness of smoking toxicity in an underappreciated area. Int J Environ Res Public Health, 2010. 7(12): p. 4111-26.

146. Bronner Murrison, L., et al., Tobacco Smoking and Tuberculosis among Men Living with HIV in Johannesburg, South Africa: A Case-Control Study. PLoS One, 2016. 11(11): p. e0167133.

147. Morgan, M.J. and Z.G. Liu, Crosstalk of reactive oxygen species and NF-kappaB signaling. Cell Res, 2011. 21(1): p. 103-15.

148. Braunersreuther, V., F. Mach, and S. Steffens, The specific role of chemokines in atherosclerosis. Thromb Haemost, 2007. 97(5): p. 714-21.

149. Ivanov, A.V., et al., Oxidative Stress during HIV Infection: Mechanisms and Consequences. Oxid Med Cell Longev, 2016. 2016: p. 8910396.

150. Price, T.O., et al., HIV-1 viral proteins gp120 and Tat induce oxidative stress in brain endothelial cells. Brain Res, 2005. 1045(1-2): p. 57-63. 
151. Deshmane, S.L., et al., Activation of the oxidative stress pathway by HIV-1 Vpr leads to induction of hypoxia-inducible factor lalpha expression. J Biol Chem, 2009. 284(17): p. 11364-73.

152. Morris, A., et al., HIV and chronic obstructive pulmonary disease: is it worse and why? Proc Am Thorac Soc, 2011. 8(3): p. 320-5.

153. Ande, A., et al., Effect of mild-to-moderate smoking on viral load, cytokines, oxidative stress, and cytochrome P450 enzymes in HIV-infected individuals. PLoS One, 2015. 10(4): p. e0122402.

154. Rao, P., et al., Effects of Cigarette Smoke Condensate on Oxidative Stress, Apoptotic Cell Death, and HIV Replication in Human Monocytic Cells. PLoS One, 2016. 11(5): p. e0155791.

155. Earla, R., et al., Enhanced nicotine metabolism in HIV-1-positive smokers compared with HIV-negative smokers: simultaneous determination of nicotine and its four metabolites in their plasma using a simple and sensitive electrospray ionization liquid chromatography-tandem mass spectrometry technique. Drug Metab Dispos, 2014. 42(2): p. 282-93.

156. Feldman, D.N., et al., CYPIA1 genotype modifies the impact of smoking on effectiveness of HAART among women. AIDS Educ Prev, 2009. 21(3 Suppl): $\mathrm{p}$. 81-93.

157. Zhao, L., et al., Mechanisms and genes involved in enhancement of HIV infectivity by tobacco smoke. Toxicology, 2010. 278(2): p. 242-8.

158. Feldman, J.G., et al., Association of cigarette smoking with HIV prognosis among women in the HAART era: a report from the women's interagency HIV study. Am J Public Health, 2006. 96(6): p. 1060-5.

159. Ande, A., et al., Tobacco smoking effect on HIV-1 pathogenesis: role of cytochrome P450 isozymes. Expert Opin Drug Metab Toxicol, 2013. 9(11): p. 1453-64.

160. Miguez-Burbano, M.J., et al., Impact of tobacco use on the development of opportunistic respiratory infections in HIV seropositive patients on antiretroviral therapy. Addict Biol, 2003. 8(1): p. 39-43.

161. Walubo, A., The role of cytochrome P450 in antiretroviral drug interactions. Expert Opin Drug Metab Toxicol, 2007. 3(4): p. 583-98.

162. Pal, D., et al., Efflux transporters- and cytochrome P-450-mediated interactions between drugs of abuse and antiretrovirals. Life Sci, 2011. 88(21-22): p. 959-71.

163. Tartaglia, E., et al., Prevalence of HPV infection among HIV-positive and HIVnegative women in Central/Eastern Italy: Strategies of prevention. Oncol Lett, 2017. 14(6): p. 7629-7635.

164. Veldhuijzen, N.J., et al., The epidemiology of human papillomavirus infection in $H I V$-positive and HIV-negative high-risk women in Kigali, Rwanda. BMC Infect Dis, 2011. 11: p. 333.

165. Badial, R.M., et al., Detection and genotyping of human papillomavirus (HPV) in $H I V$-infected women and its relationship with HPV/HIV co-infection. Medicine (Baltimore), 2018. 97(14): p. e9545.

166. McKenzie, N.D., et al., HPV in HIV-Infected Women: Implications for Primary Prevention. Front Oncol, 2014. 4: p. 179. 
167. Joshi, S., et al., Human papillomavirus infection among human immunodeficiency virus-infected women in Maharashtra, India. Vaccine, 2014. 32(9): p. 1079-85.

168. Ramogola-Masire, D., et al., Subtype distribution of human papillomavirus in $H I V$-infected women with cervical intraepithelial neoplasia stages 2 and 3 in Botswana. Int J Gynecol Pathol, 2011. 30(6): p. 591-6.

169. Edwards, R.P., et al., T lymphocytes infiltrating advanced grades of cervical neoplasia. CD8-positive cells are recruited to invasion. Cancer, 1995. 76(8): $\mathrm{p}$. 1411-5.

170. Luque, A.E., L.M. Demeter, and R.C. Reichman, Association of human papillomavirus infection and disease with magnitude of human immunodeficiency virus type 1 (HIV-1) RNA plasma level among women with HIV-1 infection. J Infect Dis, 1999. 179(6): p. 1405-9.

171. Gage, J.R., et al., Effects of human papillomavirus-associated cells on human immunodeficiency virus gene expression. Obstet Gynecol, 2000. 96(6): p. 879-85.

172. Massad, L.S., et al., Cervical cancer incidence after up to 20 years of observation among women with HIV. Int J Cancer, 2017. 141(8): p. 1561-1565.

173. Chen, C.H., et al., Risk of cancer among HIV-infected patients from a populationbased nested case-control study: implications for cancer prevention. BMC Cancer, 2015. 15: p. 133.

174. Massad, L.S., et al., Long-term incidence of cervical cancer in women with human immunodeficiency virus. Cancer, 2009. 115(3): p. 524-30.

175. Chaturvedi, A.K., et al., Risk of human papillomavirus-associated cancers among persons with AIDS. J Natl Cancer Inst, 2009. 101(16): p. 1120-30.

176. Engels, E.A., et al., Trends in cancer risk among people with AIDS in the United States 1980-2002. AIDS, 2006. 20(12): p. 1645-54.

177. Clifford, G.M., et al., Human papillomavirus types among women infected with HIV: a meta-analysis. AIDS, 2006. 20(18): p. 2337-44.

178. Frisch, M., R.J. Biggar, and J.J. Goedert, Human papillomavirus-associated cancers in patients with human immunodeficiency virus infection and acquired immunodeficiency syndrome. J Natl Cancer Inst, 2000. 92(18): p. 1500-10.

179. Ortiz, A.P., et al., Prevalence and correlates of cervical HPV infection in a clinicbased sample of HIV-positive Hispanic women. Papillomavirus Res, 2017. 4: p. 39-44.

180. Menon, S., et al., Epidemiology of HPV Genotypes among HIV Positive Women in Kenya: A Systematic Review and Meta-Analysis. PLoS One, 2016. 11(10): p. e0163965.

181. Clifford, G.M., et al., Effect of HIV Infection on Human Papillomavirus Types Causing Invasive Cervical Cancer in Africa. J Acquir Immune Defic Syndr, 2016. 73(3): p. 332-339.

182. Marchetti, G., et al., HPV Infection in a Cohort of HIV-Positive Men and Women: Prevalence of Oncogenic Genotypes and Predictors of Mucosal Damage at Genital and Oral Sites. J Sex Transm Dis, 2013. 2013: p. 915169.

183. Sahasrabuddhe, V.V., et al., Prevalence and distribution of HPV genotypes among HIV-infected women in Zambia. Br J Cancer, 2007. 96(9): p. 1480-3. 
184. Lai, D., et al., Localization of HPV-18 E2 at mitochondrial membranes induces ROS release and modulates host cell metabolism. PLoS One, 2013. 8(9): p. e75625.

185. Cruz-Gregorio, A., et al., Human Papillomavirus Types 16 and 18 Earlyexpressed Proteins Differentially Modulate the Cellular Redox State and DNA Damage. Int J Biol Sci, 2018. 14(1): p. 21-35.

186. Williams, V.M., et al., Human papillomavirus type 16 E6* induces oxidative stress and DNA damage. J Virol, 2014. 88(12): p. 6751-61.

187. De Marco, F., et al., Oxidative stress in HPV-driven viral carcinogenesis: redox proteomics analysis of HPV-16 dysplastic and neoplastic tissues. PLoS One, 2012. 7(3): p. e34366.

188. Duh, E.J., et al., Tumor necrosis factor alpha activates human immunodeficiency virus type 1 through induction of nuclear factor binding to the NF-kappa B sites in the long terminal repeat. Proc Natl Acad Sci U S A, 1989. 86(15): p. 5974-8.

189. Poli, G., et al., Interleukin 6 induces human immunodeficiency virus expression in infected monocytic cells alone and in synergy with tumor necrosis factor alpha by transcriptional and post-transcriptional mechanisms. J Exp Med, 1990. 172(1): p. 151-8.

190. Piette, J. and S. Legrand-Poels, HIV-1 reactivation after an oxidative stress mediated by different reactive oxygen species. Chem Biol Interact, 1994. 91(2-3): p. 79-89.

191. Legrand-Poels, S., et al., Activation of human immunodeficiency virus type 1 by oxidative stress. AIDS Res Hum Retroviruses, 1990. 6(12): p. 1389-97.

192. Schreck, R., P. Rieber, and P.A. Baeuerle, Reactive oxygen intermediates as apparently widely used messengers in the activation of the NF-kappa B transcription factor and HIV-1. EMBO J, 1991. 10(8): p. 2247-58.

193. Bratcher, L.F. and V.V. Sahasrabuddhe, The impact of antiretroviral therapy on HPV and cervical intraepithelial neoplasia: current evidence and directions for future research. Infect Agent Cancer, 2010. 5: p. 8.

194. Blitz, S., et al., Evaluation of HIV and highly active antiretroviral therapy on the natural history of human papillomavirus infection and cervical cytopathologic findings in HIV-positive and high-risk HIV-negative women. J Infect Dis, 2013. 208(3): p. 454-62.

195. Clifford, G.M., et al., Immunodeficiency and the risk of cervical intraepithelial neoplasia 2/3 and cervical cancer: A nested case-control study in the Swiss HIV cohort study. Int J Cancer, 2016. 138(7): p. 1732-40.

196. Konopnicki, D., et al., Sustained viral suppression and higher CD4+ T-cell count reduces the risk of persistent cervical high-risk human papillomavirus infection in HIV-positive women. J Infect Dis, 2013. 207(11): p. 1723-9.

197. Kelly, H., et al., Association of antiretroviral therapy with high-risk human papillomavirus, cervical intraepithelial neoplasia, and invasive cervical cancer in women living with HIV: a systematic review and meta-analysis. Lancet HIV, 2018. 5(1): p. e45-e58.

198. Cobucci, R.N., et al., Assessing the impact of HAART on the incidence of defining and non-defining AIDS cancers among patients with HIV/AIDS: a systematic review. J Infect Public Health, 2015. 8(1): p. 1-10. 
199. Gisler, V., et al., AIDS defining opportunistic infections in patients with high CD4 counts in the combination antiretroviral therapy (cART) era: things ain't what they used to be. J Int AIDS Soc, 2014. 17(4 Suppl 3): p. 19621.

200. Qiu, F., et al., Impacts of cigarette smoking on immune responsiveness: Up and down or upside down? Oncotarget, 2017. 8(1): p. 268-284.

201. Lee, J., V. Taneja, and R. Vassallo, Cigarette smoking and inflammation: cellular and molecular mechanisms. J Dent Res, 2012. 91(2): p. 142-9.

202. Ozguner, F., A. Koyu, and G. Cesur, Active smoking causes oxidative stress and decreases blood melatonin levels. Toxicol Ind Health, 2005. 21(1-2): p. 21-6.

203. Kamceva, G., et al., Cigarette Smoking and Oxidative Stress in Patients with Coronary Artery Disease. Open Access Maced J Med Sci, 2016. 4(4): p. 636-640.

204. Xi, L.F., et al., Relationship between cigarette smoking and human papilloma virus types 16 and 18 DNA load. Cancer Epidemiol Biomarkers Prev, 2009. 18(12): p. 3490-6.

205. Cory, T.J., et al., Overcoming pharmacologic sanctuaries. Curr Opin HIV AIDS, 2013. 8(3): p. 190-5.

206. Kumar, A. and G. Herbein, The macrophage: a therapeutic target in HIV-I infection. Mol Cell Ther, 2014. 2: p. 10.

207. Kilareski, E.M., et al., Regulation of HIV-1 transcription in cells of the monocytemacrophage lineage. Retrovirology, 2009. 6: p. 118.

208. Lewin, S.R., et al., Constitutive expression of p50 homodimer in freshly isolated human monocytes decreases with in vitro and in vivo differentiation: a possible mechanism influencing human immunodeficiency virus replication in monocytes and mature macrophages. J Virol, 1997. 71(3): p. 2114-9.

209. Dong, C., C. Kwas, and L. Wu, Transcriptional restriction of human immunodeficiency virus type 1 gene expression in undifferentiated primary monocytes. J Virol, 2009. 83(8): p. 3518-27.

210. Filippova, M., et al., Cellular levels of oxidative stress affect the response of cervical cancer cells to chemotherapeutic agents. Biomed Res Int, 2014. 2014: p. 574659.

211. Haque, S., et al., Monocyte-derived exosomes upon exposure to cigarette smoke condensate alter their characteristics and show protective effect against cytotoxicity and HIV-1 replication. Scientific Reports, 2017. 7(1): p. 16120.

212. Humans, I.W.G.o.t.E.o.C.R.t., Tobacco smoke and involuntary smoking. IARC Monogr Eval Carcinog Risks Hum, 2004. 83: p. 1-1438.

213. Hoffmann, D., G. Rathkamp, and J. Rubin, Chemical studies on tobacco smoke. II. Comparison of the yields of several selected components in the smoke from five major Turkish tobacco varieties. Food Cosmet Toxicol, 1967. 5(1): p. 37-8.

214. Hecht, S.S., I. Stepanov, and S.G. Carmella, Exposure and Metabolic Activation Biomarkers of Carcinogenic Tobacco-Specific Nitrosamines. Acc Chem Res, 2016. 49(1): p. 106-14.

215. Wang, G.Z., et al., The chemokine CXCL13 in lung cancers associated with environmental polycyclic aromatic hydrocarbons pollution. Elife, 2015. 4.

216. Siddens, L.K., et al., Polycyclic aromatic hydrocarbons as skin carcinogens: comparison of benzo[a] pyrene, dibenzo[def,p] chrysene and three environmental mixtures in the FVB/N mouse. Toxicol Appl Pharmacol, 2012. 264(3): p. 377-86. 
217. Chuang, C.Y., et al., BPDE-like DNA adduct level in oral tissue may act as a risk biomarker of oral cancer. Arch Oral Biol, 2013. 58(1): p. 102-9.

218. McCarty, K.M., et al., PAH-DNA adducts, cigarette smoking, GST polymorphisms, and breast cancer risk. Environ Health Perspect, 2009. 117(4): p. 552-8.

219. Vu, A.T., et al., Polycyclic Aromatic Hydrocarbons in the Mainstream Smoke of Popular U.S. Cigarettes. Chem Res Toxicol, 2015. 28(8): p. 1616-26.

220. Shimada, T. and Y. Fujii-Kuriyama, Metabolic activation of polycyclic aromatic hydrocarbons to carcinogens by cytochromes P450 1A1 and 1B1. Cancer Sci, 2004. 95(1): p. 1-6.

221. Conney, A.H., Induction of microsomal enzymes by foreign chemicals and carcinogenesis by polycyclic aromatic hydrocarbons: G. H. A. Clowes Memorial Lecture. Cancer Res, 1982. 42(12): p. 4875-917.

222. Henkler, F., K. Stolpmann, and A. Luch, Exposure to polycyclic aromatic hydrocarbons: bulky DNA adducts and cellular responses. EXS, 2012. 101: p. 107-31.

223. Goldman, R., et al., Smoking increases carcinogenic polycyclic aromatic hydrocarbons in human lung tissue. Cancer Res, 2001. 61(17): p. 6367-71.

224. Sadeu, J.C. and W.G. Foster, The cigarette smoke constituent benzo[a]pyrene disrupts metabolic enzyme, and apoptosis pathway member gene expression in ovarian follicles. Reprod Toxicol, 2013. 40: p. 52-9.

225. Van Tiem, L.A. and R.T. Di Giulio, AHR2 knockdown prevents PAH-mediated cardiac toxicity and XRE- and ARE-associated gene induction in zebrafish (Danio rerio). Toxicol Appl Pharmacol, 2011. 254(3): p. 280-7.

226. Nebert, D.W., et al., Role of aryl hydrocarbon receptor-mediated induction of the CYPl enzymes in environmental toxicity and cancer. J Biol Chem, 2004. 279(23): p. 23847-50.

227. Wohak, L.E., et al., Carcinogenic polycyclic aromatic hydrocarbons induce CYP1A1 in human cells via a p53-dependent mechanism. Arch Toxicol, 2016. 90(2): p. 291-304.

228. Zhong, Y., et al., Metabolism of [D10]phenanthrene to tetraols in smokers for potential lung cancer susceptibility assessment: comparison of oral and inhalation routes of administration. J Pharmacol Exp Ther, 2011. 338(1): p. 35361.

229. Bagchi, M., et al., Protective effect of melatonin on naphthalene-induced oxidative stress and DNA damage in cultured macrophage J774A.1 cells. Mol Cell Biochem, 2001. 221(1-2): p. 49-55.

230. Jin, M., et al., A LC-MS/MS method for concurrent determination of nicotine metabolites and role of CYP2A6 in nicotine metabolism in U937 macrophages: implications in oxidative stress in HIV + smokers. J Neuroimmune Pharmacol, 2012. 7(1): p. 289-99.

231. Pierson, T., J. McArthur, and R.F. Siliciano, Reservoirs for HIV-1: mechanisms for viral persistence in the presence of antiviral immune responses and antiretroviral therapy. Annu Rev Immunol, 2000. 18: p. 665-708.

232. Shimada, T., et al., Metabolic activation of polycyclic aromatic hydrocarbons and other procarcinogens by cytochromes P450 1A1 and P450 1B1 allelic variants 
and other human cytochromes P450 in Salmonella typhimurium NM2009. Drug Metab Dispos, 2001. 29(9): p. 1176-82.

233. Jablonowska-Fudzinska, D., A. Marszalek, and L. Szylberg, [Tobacco smoking as a cofactor for the development of cervical cancer]. Przegl Lek, 2015. 72(3): p. 103-5.

234. Pfeifer, G.P., et al., Tobacco smoke carcinogens, DNA damage and p53 mutations in smoking-associated cancers. Oncogene, 2002. 21(48): p. 7435-51.

235. Zhu, W., et al., Curcumin and vitamin E protect against adverse effects of benzo[a]pyrene in lung epithelial cells. PLoS One, 2014. 9(3): p. e92992.

236. Hardin, J.A., F. Hinoshita, and D.H. Sherr, Mechanisms by which benzo[a]pyrene, an environmental carcinogen, suppresses B cell lymphopoiesis. Toxicol Appl Pharmacol, 1992. 117(2): p. 155-64.

237. Gao, D., et al., Chronic exposure to low benzo[a]pyrene level causes neurodegenerative disease-like syndromes in zebrafish (Danio rerio). Aquat Toxicol, 2015. 167: p. 200-8.

238. Einaudi, L., et al., In vivo exposure to benzo(a)pyrene induces significant DNA damage in mouse oocytes and cumulus cells. Hum Reprod, 2014. 29(3): p. 54854.

239. Santacroce, M.P., et al., Implications for chronic toxicity of benzo[a]pyrene in sea bream cultured hepatocytes: Cytotoxicity, inflammation, and cancerogenesis. Environ Toxicol, 2015. 30(9): p. 1045-62.

240. Sugimoto, K., et al., Frequent mutations in the p53 gene in human myeloid leukemia cell lines. Blood, 1992. 79(9): p. 2378-83.

241. Krais, A.M., et al., The impact of 553 on DNA damage and metabolic activation of the environmental carcinogen benzo[a]pyrene: effects in Trp53(+/+), Trp53(+/-) and Trp53(-/-) mice. Arch Toxicol, 2016. 90(4): p. 839-51.

242. Sablina, A.A., et al., The antioxidant function of the p53 tumor suppressor. Nat Med, 2005. 11(12): p. 1306-13.

243. Andrieux, L., et al., Aryl hydrocarbon receptor activation and cytochrome P450 $1 A$ induction by the mitogen-activated protein kinase inhibitor U0126 in hepatocytes. Mol Pharmacol, 2004. 65(4): p. 934-43.

244. Kucab, J.E., et al., TP53 mutations induced by BPDE in Xpa-WT and Xpa-Null human TP53 knock-in (Hupki) mouse embryo fibroblasts. Mutat Res, 2015. 773: p. 48-62.

245. Chang, H., et al., Preferential induction of CYP1A1 and CYP1B1 in CCSPpositive cells. Toxicol Sci, 2006. 89(1): p. 205-13.

246. Harrigan, J.A., et al., Tissue specific induction of cytochrome P450 (CYP) $1 \mathrm{Al}$ and $1 B 1$ in rat liver and lung following in vitro (tissue slice) and in vivo exposure to benzo(a)pyrene. Toxicol In Vitro, 2006. 20(4): p. 426-38.

247. Houser, W.H., A. Raha, and M. Vickers, Induction of CYP1A1 gene expression in H4-II-E rat hepatoma cells by benzo[e]pyrene. Mol Carcinog, 1992. 5(3): p. 2327.

248. Matsunawa, M., et al., Vitamin D receptor activation enhances benzo[a]pyrene metabolism via CYP1A1 expression in macrophages. Drug Metab Dispos, 2012. 40(11): p. 2059-66. 
249. Arlt, V.M., et al., Pulmonary Inflammation Impacts on CYP1A1-Mediated Respiratory Tract DNA Damage Induced by the Carcinogenic Air Pollutant Benzo[a]pyrene. Toxicol Sci, 2015. 146(2): p. 213-25.

250. Plottner, S., et al., Evaluation of time dependence and interindividual differences in benzo[a]pyrene-mediated CYP1A1 induction and genotoxicity in porcine urinary bladder cell cultures. J Toxicol Environ Health A, 2008. 71(13-14): p. 969-75.

251. Iqbal, J., et al., Smoke carcinogens cause bone loss through the aryl hydrocarbon receptor and induction of Cypl enzymes. Proc Natl Acad Sci U S A, 2013. 110(27): p. 11115-20.

252. Jin, M., et al., Effect of alcohol on drug efflux protein and drug metabolic enzymes in U937 macrophages. Alcohol Clin Exp Res, 2011. 35(1): p. 132-9.

253. Luckert, C., et al., Polycyclic aromatic hydrocarbons stimulate human CYP3A4 promoter activity via PXR. Toxicol Lett, 2013. 222(2): p. 180-8.

254. Kumagai, T., et al., Polycyclic aromatic hydrocarbons activate CYP3A4 gene transcription through human pregnane X receptor. Drug Metab Pharmacokinet, 2012. 27(2): p. 200-6.

255. Gupta, R.K., et al., Oxidative stress and antioxidants in disease and cancer: a review. Asian Pac J Cancer Prev, 2014. 15(11): p. 4405-9.

256. Karihtala, P. and Y. Soini, Reactive oxygen species and antioxidant mechanisms in human tissues and their relation to malignancies. APMIS, 2007. 115(2): p. 81103.

257. Li, N., et al., Nrf2 is a key transcription factor that regulates antioxidant defense in macrophages and epithelial cells: protecting against the proinflammatory and oxidizing effects of diesel exhaust chemicals. J Immunol, 2004. 173(5): p. 346781.

258. Aleksunes, L.M. and J.E. Manautou, Emerging role of Nrf2 in protecting against hepatic and gastrointestinal disease. Toxicol Pathol, 2007. 35(4): p. 459-73.

259. Salas, V.M. and S.W. Burchiel, Apoptosis in Daudi human B cells in response to benzo[a]pyrene and benzo[a]pyrene-7,8-dihydrodiol. Toxicol Appl Pharmacol, 1998. 151(2): p. 367-76.

260. Xiao, H., et al., Benzo[a]pyrene-7,8-diol-9,10-epoxide causes caspase-mediated apoptosis in H460 human lung cancer cell line. Cell Cycle, 2007. 6(22): p. 282634.

261. Chen, S., et al., The role of the Ah receptor and p38 in benzo[a]pyrene-7,8dihydrodiol and benzo[a]pyrene-7,8-dihydrodiol-9,10-epoxide-induced apoptosis. J Biol Chem, 2003. 278(21): p. 19526-33.

262. Kim, J.Y., et al., Benzo[a]pyrene induces apoptosis in RL95-2 human endometrial cancer cells by cytochrome P450 1A1 activation. Endocrinology, 2007. 148(10): p. 5112-22.

263. Circu, M.L. and T.Y. Aw, Reactive oxygen species, cellular redox systems, and apoptosis. Free Radic Biol Med, 2010. 48(6): p. 749-62.

264. Valiathan, R., et al., Tobacco smoking increases immune activation and impairs T-cell function in HIV infected patients on antiretrovirals: a cross-sectional pilot study. PLoS One, 2014. 9(5): p. e97698. 
265. Wojna, V., et al., Associations of cigarette smoking with viral immune and cognitive function in human immunodeficiency virus-seropositive women. $\mathrm{J}$ Neurovirol, 2007. 13(6): p. 561-8.

266. Rao, P.S. and S. Kumar, Polycyclic aromatic hydrocarbons and cytochrome P450 in HIV pathogenesis. Front Microbiol, 2015. 6: p. 550.

267. Ali, R., et al., Amelioration of Benzo[a]pyrene-induced oxidative stress and pulmonary toxicity by Naringenin in Wistar rats: A plausible role of $C O X-2$ and NF-kappaB. Hum Exp Toxicol, 2017. 36(4): p. 349-364.

268. Ji, K., et al., Benzo[a]pyrene induces oxidative stress and endothelial progenitor cell dysfunction via the activation of the NF-kappaB pathway. Int J Mol Med, 2013. 31(4): p. 922-30.

269. Liamin, M., et al., Benzo[a]pyrene-induced DNA damage associated with mutagenesis in primary human activated T lymphocytes. Biochem Pharmacol, 2017. 137: p. 113-124.

270. Xia, B., et al., Biotin-mediated epigenetic modifications: Potential defense against the carcinogenicity of benzo[a]pyrene. Toxicol Lett, 2016. 241: p. 216-24.

271. Li, E., et al., Macrophages promote benzopyrene-induced tumor transformation of human bronchial epithelial cells by activation of NF-kappaB and STAT3 signaling in a bionic airway chip culture and in animal models. Oncotarget, 2015. 6(11): p. 8900-13.

272. Phillips, T.D., et al., Mechanistic relationships between hepatic genotoxicity and carcinogenicity in male B6C3F1 mice treated with polycyclic aromatic hydrocarbon mixtures. Arch Toxicol, 2015. 89(6): p. 967-77.

273. Ranjit, S., et al., Effect of Polyaryl Hydrocarbons on Cytotoxicity in Monocytic Cells: Potential Role of Cytochromes P450 and Oxidative Stress Pathways. PLoS One, 2016. 11(9): p. e0163827.

274. Xue, W. and D. Warshawsky, Metabolic activation of polycyclic and heterocyclic aromatic hydrocarbons and DNA damage: a review. Toxicol Appl Pharmacol, 2005. 206(1): p. 73-93.

275. Briede, J.J., et al., In vitro and in vivo studies on oxygen free radical and DNA adduct formation in rat lung and liver during benzo[a]pyrene metabolism. Free Radic Res, 2004. 38(9): p. 995-1002.

276. Costa, C., et al., Exposure of human skin to benzo[a]pyrene: role of CYP1A1 and aryl hydrocarbon receptor in oxidative stress generation. Toxicology, 2010. 271(3): p. 83-6.

277. Ryter, S.W., et al., Mechanisms of cell death in oxidative stress. Antioxid Redox Signal, 2007. 9(1): p. 49-89.

278. Rahal, A., et al., Oxidative stress, prooxidants, and antioxidants: the interplay. Biomed Res Int, 2014. 2014: p. 761264.

279. Aquaro, S., et al., The contribution of peroxynitrite generation in HIV replication in human primary macrophages. Retrovirology, 2007. 4: p. 76.

280. Westendorp, M.O., et al., HIV-1 Tat potentiates TNF-induced NF-kappa B activation and cytotoxicity by altering the cellular redox state. EMBO J, 1995. 14(3): p. 546-54. 
281. Pyo, C.W., et al., Reactive oxygen species activate HIV long terminal repeat via post-translational control of NF-kappaB. Biochem Biophys Res Commun, 2008. 376(1): p. 180-5.

282. Oeckinghaus, A. and S. Ghosh, The NF-kappaB family of transcription factors and its regulation. Cold Spring Harb Perspect Biol, 2009. 1(4): p. a000034.

283. Hoffmann, A. and D. Baltimore, Circuitry of nuclear factor kappaB signaling. Immunol Rev, 2006. 210: p. 171-86.

284. Hayden, M.S., A.P. West, and S. Ghosh, NF-kappaB and the immune response. Oncogene, 2006. 25(51): p. 6758-80.

285. Nookala, A.R., et al., HIV-1 Tat-mediated induction of CCL5 in astrocytes involves NF-kappaB, AP-1, C/EBPalpha and C/EBPgamma transcription factors and JAK, PI3K/Akt and p38 MAPK signaling pathways. PLoS One, 2013. 8(11): p. e78855.

286. Shah, A., et al., HIV-1 gp120 induces expression of IL-6 through a nuclear factorkappa B-dependent mechanism: suppression by gp 120 specific small interfering RNA. PLoS One, 2011. 6(6): p. e21261.

287. Zordoky, B.N. and A.O. El-Kadi, Role of NF-kappaB in the regulation of cytochrome P450 enzymes. Curr Drug Metab, 2009. 10(2): p. 164-78.

288. Zhang, J., et al., ROS and ROS-Mediated Cellular Signaling. Oxid Med Cell Longev, 2016. 2016: p. 4350965.

289. Karin, M. and Y. Ben-Neriah, Phosphorylation meets ubiquitination: the control of NF-[kappa] B activity. Annu Rev Immunol, 2000. 18: p. 621-63.

290. Hiscott, J., H. Kwon, and P. Genin, Hostile takeovers: viral appropriation of the NF-kappaB pathway. J Clin Invest, 2001. 107(2): p. 143-51.

291. Israel, N., et al., Redox status of cells influences constitutive or induced NF-kappa $B$ translocation and HIV long terminal repeat activity in human T and monocytic cell lines. J Immunol, 1992. 149(10): p. 3386-93.

292. Koppensteiner, H., R. Brack-Werner, and M. Schindler, Macrophages and their relevance in Human Immunodeficiency Virus Type I infection. Retrovirology, 2012. 9: p. 82.

293. Sattentau, Q.J. and M. Stevenson, Macrophages and HIV-1: An Unhealthy Constellation. Cell Host Microbe, 2016. 19(3): p. 304-10.

294. Atluri, V.S., et al., Effect of human immunodeficiency virus on blood-brain barrier integrity and function: an update. Front Cell Neurosci, 2015. 9: p. 212.

295. Nabha, L., L. Duong, and J. Timpone, HIV-associated neurocognitive disorders: perspective on management strategies. Drugs, 2013. 73(9): p. 893-905.

296. Ciolino, H.P., P.J. Daschner, and G.C. Yeh, Resveratrol inhibits transcription of CYPIA1 in vitro by preventing activation of the aryl hydrocarbon receptor. Cancer Res, 1998. 58(24): p. 5707-12.

297. Chang, C.Y. and A. Puga, Constitutive activation of the aromatic hydrocarbon receptor. Mol Cell Biol, 1998. 18(1): p. 525-35.

298. Treitinger, A., et al., Decreased antioxidant defence in individuals infected by the human immunodeficiency virus. Eur J Clin Invest, 2000. 30(5): p. 454-9.

299. Wu, Z., et al., Z-Ligustilide inhibits benzo(a)pyrene-induced CYP1A1 upregulation in cultured human keratinocytes via ROS-dependent Nrf2 activation. Exp Dermatol, 2014. 23(4): p. 260-5. 
300. Bao, H., M. Vepakomma, and M.A. Sarkar, Benzo(a)pyrene exposure induces CYPIAl activity and expression in human endometrial cells. J Steroid Biochem Mol Biol, 2002. 81(1): p. 37-45.

301. Billiard, S.M., et al., The role of the aryl hydrocarbon receptor pathway in mediating synergistic developmental toxicity of polycyclic aromatic hydrocarbons to zebrafish. Toxicol Sci, 2006. 92(2): p. 526-36.

302. Scheel, J., et al., Variability of the human aryl hydrocarbon receptor nuclear translocator (ARNT) gene. J Hum Genet, 2002. 47(5): p. 217-24.

303. Mohammadi-Bardbori, A., Assay for quantitative determination of CYP1A1 enzyme activity using 7-Ethoxyresorufin as standard substrate (EROD assay). 2014.

304. Zangar, R.C., D.R. Davydov, and S. Verma, Mechanisms that regulate production of reactive oxygen species by cytochrome P450. Toxicol Appl Pharmacol, 2004. 199(3): p. 316-31.

305. Wu, J.P., et al., Involvement of oxidative stress and activation of aryl hydrocarbon receptor in elevation of CYPIA1 expression and activity in lung cells and tissues by arsenic: an in vitro and in vivo study. Toxicol Sci, 2009. 107(2): p. 385-93.

306. Birben, E., et al., Oxidative stress and antioxidant defense. World Allergy Organ J, 2012. 5(1): p. 9-19.

307. Nguyen, T., P. Nioi, and C.B. Pickett, The Nrf2-antioxidant response element signaling pathway and its activation by oxidative stress. J Biol Chem, 2009. 284(20): p. 13291-5.

308. Ma, Q., Role of nrf2 in oxidative stress and toxicity. Annu Rev Pharmacol Toxicol, 2013. 53: p. 401-26.

309. Pagliari, L.J., et al., Macrophages require constitutive NF-kappaB activation to maintain Al expression and mitochondrial homeostasis. Mol Cell Biol, 2000. 20(23): p. 8855-65.

310. Stroud, J.C., et al., Structural basis of HIV-1 activation by NF-kappaB--a higherorder complex of p50:RelA bound to the HIV-1 LTR. J Mol Biol, 2009. 393(1): p. 98-112.

311. Montorfano, I., et al., Oxidative stress mediates the conversion of endothelial cells into myofibroblasts via a TGF-betal and TGF-beta2-dependent pathway. Lab Invest, 2014. 94(10): p. 1068-82.

312. Liu, Q., et al., SC-514, a selective inhibitor of IKKbeta attenuates RANKLinduced osteoclastogenesis and NF-kappaB activation. Biochem Pharmacol, 2013. 86(12): p. 1775-83.

313. Kishore, N., et al., A selective IKK-2 inhibitor blocks NF-kappa B-dependent gene expression in interleukin-1 beta-stimulated synovial fibroblasts. J Biol Chem, 2003. 278(35): p. 32861-71.

314. Chan, J.K. and W.C. Greene, Dynamic roles for NF-kappaB in HTLV-I and HIV-1 retroviral pathogenesis. Immunol Rev, 2012. 246(1): p. 286-310.

315. El-Amine, R., et al., HIV-1 Tat protein induces DNA damage in human peripheral blood B-lymphocytes via mitochondrial ROS production. Redox Biol, 2018. 15: p. 97-108.

316. Wang, T., et al., Transfer of intracellular HIV Nef to endothelium causes endothelial dysfunction. PLoS One, 2014. 9(3): p. e91063. 
317. Capone, C., et al., A role for spermine oxidase as a mediator of reactive oxygen species production in HIV-Tat-induced neuronal toxicity. Free Radic Biol Med, 2013. 63: p. 99-107.

318. Wu, R.F., et al., HIV-1 Tat activates dual Nox pathways leading to independent activation of ERK and JNK MAP kinases. J Biol Chem, 2007. 282(52): p. 374129.

319. Pandhare, J., et al., A Novel Role of Proline Oxidase in HIV-1 Envelope Glycoprotein-induced Neuronal Autophagy. J Biol Chem, 2015. 290(42): p. 25439-51.

320. Olivetta, E., et al., HIV-1 Nef induces 447 (phox) phosphorylation leading to a rapid superoxide anion release from the U937 human monoblastic cell line. J Cell Biochem, 2009. 106(5): p. 812-22.

321. Murphy, K.M., et al., Effects of the tat and nef gene products of human immunodeficiency virus type 1 (HIV-1) on transcription controlled by the HIV-1 long terminal repeat and on cell growth in macrophages. J Virol, 1993. 67(12): p. 6956-64.

322. Clifford, G.M., S. Tully, and S. Franceschi, Carcinogenicity of Human Papillomavirus (HPV) Types in HIV-Positive Women: A Meta-Analysis From HPV Infection to Cervical Cancer. Clin Infect Dis, 2017. 64(9): p. 1228-1235.

323. Stuardo, V., et al., Human papillomavirus infection in HIV-1 infected women in Catalonia (Spain): implications for prevention of cervical cancer. PLoS One, 2012. 7(10): p. e47755.

324. Brickman, C. and J.M. Palefsky, Human papillomavirus in the HIV-infected host: epidemiology and pathogenesis in the antiretroviral era. Curr HIV/AIDS Rep, 2015. 12(1): p. 6-15.

325. Maiman, M., et al., Cervical cancer as an AIDS-defining illness. Obstetrics \& Gynecology, 1997. 89(1): p. 76-80.

326. Manju, V., V. Balasubramanian, and N. Nalini, Oxidative stress and tumor markers in cervical cancer patients. J Biochem Mol Biol Biophys, 2002. 6(6): p. 387-90.

327. De Marco, F., Oxidative stress and HPV carcinogenesis. Viruses, 2013. 5(2): p. 708-31.

328. Naidu, M.S., et al., Oxidative stress and antioxidant status in cervical cancer patients. Indian J Clin Biochem, 2007. 22(2): p. 140-4.

329. Manju, V., J. Kalaivani Sailaja, and N. Nalini, Circulating lipid peroxidation and antioxidant status in cervical cancer patients: a case-control study. Clin Biochem, 2002. 35(8): p. 621-5.

330. Goncalves, T.L., et al., Involvement of oxidative stress in the pre-malignant and malignant states of cervical cancer in women. Clin Biochem, 2005. 38(12): p. 1071-5.

331. Yim, E.K. and J.S. Park, The role of HPV E6 and E7 oncoproteins in HPVassociated cervical carcinogenesis. Cancer Res Treat, 2005. 37(6): p. 319-24.

332. Ding, B., et al., Role of 53 in antioxidant defense of HPV-positive cervical carcinoma cells following H2O2 exposure. J Cell Sci, 2007. 120(Pt 13): p. 228494. 
333. Liu, J., et al., Increased exosomal microRNA-21 and microRNA-146a levels in the cervicovaginal lavage specimens of patients with cervical cancer. Int J Mol Sci, 2014. 15(1): p. 758-73.

334. Honegger, A., et al., Silencing of human papillomavirus (HPV) E6/E7 oncogene expression affects both the contents and the amounts of extracellular microvesicles released from HPV-positive cancer cells. Int J Cancer, 2013. 133(7): p. 1631-42.

335. Théry, C., L. Zitvogel, and S. Amigorena, Exosomes: composition, biogenesis and function. Nature Reviews Immunology, 2002. 2: p. 569.

336. Tkach, M. and C. Thery, Communication by Extracellular Vesicles: Where We Are and Where We Need to Go. Cell, 2016. 164(6): p. 1226-1232.

337. Harden, M.E. and K. Munger, Human papillomavirus 16 E6 and E7 oncoprotein expression alters microRNA expression in extracellular vesicles. Virology, 2017. 508: p. 63-69.

338. Khan, S., et al., Extracellular, cell-permeable survivin inhibits apoptosis while promoting proliferative and metastatic potential. Br J Cancer, 2009. 100(7): $\mathrm{p}$. 1073-86.

339. Chen Wongworawat, Y., et al., Chronic oxidative stress increases the integration frequency of foreign DNA and human papillomavirus 16 in human keratinocytes. Am J Cancer Res, 2016. 6(4): p. 764-80.

340. Williams, V.M., et al., HPV-DNA integration and carcinogenesis: putative roles for inflammation and oxidative stress. Future Virol, 2011. 6(1): p. 45-57.

341. Ranjit, S., et al., Benzo(a)pyrene in Cigarette Smoke Enhances HIV-1 Replication through NF- $\kappa B$ Activation via CYP-Mediated Oxidative Stress Pathway. Scientific Reports, 2018. 8(1): p. 10394.

342. Szatanek, R., et al., Isolation of extracellular vesicles: Determining the correct approach (Review). Int J Mol Med, 2015. 36(1): p. 11-7.

343. Didenko, V.V., 5'OH DNA breaks in apoptosis and their labeling by topoisomerase-based approach. Methods Mol Biol, 2011. 682: p. 77-87.

344. Zaman, M.S., et al., Curcumin Nanoformulation for Cervical Cancer Treatment. Sci Rep, 2016. 6: p. 20051.

345. Sikander, M., et al., Cucurbitacin D exhibits potent anti-cancer activity in cervical cancer. Sci Rep, 2016. 6: p. 36594.

346. Yallapu, M.M., M. Jaggi, and S.C. Chauhan, Curcumin nanoformulations: a future nanomedicine for cancer. Drug Discov Today, 2012. 17(1-2): p. 71-80.

347. Ali, A. and A.C. Banerjea, Curcumin inhibits HIV-1 by promoting Tat protein degradation. Sci Rep, 2016. 6: p. 27539.

348. Kumari, N., et al., Inhibition of HIV-1 by curcumin A, a novel curcumin analog. Drug Des Devel Ther, 2015. 9: p. 5051-60.

349. Staal, F.J., et al., Intracellular thiols regulate activation of nuclear factor kappa B and transcription of human immunodeficiency virus. Proc Natl Acad Sci U S A, 1990. 87(24): p. 9943-7.

350. Saccani, A., et al., Redox regulation of chemokine receptor expression. Proc Natl Acad Sci U S A, 2000. 97(6): p. 2761-6. 
351. Tuttle, D.L., et al., Expression of CCR5 increases during monocyte differentiation and directly mediates macrophage susceptibility to infection by human immunodeficiency virus type 1. J Virol, 1998. 72(6): p. 4962-9.

352. Kalebic, T., et al., Suppression of human immunodeficiency virus expression in chronically infected monocytic cells by glutathione, glutathione ester, and Nacetylcysteine. Proc Natl Acad Sci U S A, 1991. 88(3): p. 986-90.

353. Fulda, S., et al., Cellular stress responses: cell survival and cell death. Int J Cell Biol, 2010. 2010: p. 214074.

354. Xue, Y., et al., Detection of survivin expression in cervical cancer cells using molecular beacon imaging: new strategy for the diagnosis of cervical cancer. Eur J Obstet Gynecol Reprod Biol, 2011. 159(1): p. 204-8.

355. Khan, S., et al., Survivin is released from cancer cells via exosomes. Apoptosis, 2011. 16(1): p. 1-12.

356. Geleziunas, R., et al., HIV-1 Nef inhibits ASK1-dependent death signalling providing a potential mechanism for protecting the infected host cell. Nature, 2001. 410(6830): p. 834-8.

357. Piotrowska, H., M. Kucinska, and M. Murias, Expression of CYP1A1, CYP1B1 and MnSOD in a panel of human cancer cell lines. Mol Cell Biochem, 2013. 383(1-2): p. 95-102.

358. Shebl, F.M., et al., Comparison of $m R N A$ and protein measures of cytokines following vaccination with human papillomavirus-16 L1 virus-like particles. Cancer Epidemiol Biomarkers Prev, 2010. 19(4): p. 978-81.

359. Mehra, A., K.H. Lee, and V. Hatzimanikatis, Insights into the relation between $m R N A$ and protein expression patterns: I. Theoretical considerations. Biotechnol Bioeng, 2003. 84(7): p. 822-33.

360. Sarro, S.M., et al., Quantification of CD20 mRNA and protein levels in chronic lymphocytic leukemia suggests a post-transcriptional defect. Leuk Res, 2010. 34(12): p. 1670-3.

361. Farberov, L., et al., MicroRNA-mediated regulation of p21 and TASK1 cellular restriction factors enhances HIV-1 infection. J Cell Sci, 2015. 128(8): p. 1607-16.

362. Jin, C., et al., MicroRNA-181 expression regulates specific post-transcriptional level of SAMHD1 expression in vitro. Biochem Biophys Res Commun, 2014. 452(3): p. 760-7.

363. ElizabethKunchandy, M.N.A.R., Oxygen radical scavenging activity of curcumin International Journal of Pharmaceutics, 1990. 58(3): p. 237-240.

364. health, P., Research and treatment. 2018.

365. Larson, E.C., et al., Mycobacterium tuberculosis reactivates latent $\mathrm{HIV}-1$ in $\mathrm{T}$ cells in vitro. PLoS One, 2017. 12(9): p. e0185162.

366. Wang, X., et al., Alcohol potentiates HIV-1 infection of human blood mononuclear phagocytes. Alcohol Clin Exp Res, 2002. 26(12): p. 1880-6.

367. Buchacz, K., et al., Syphilis increases HIV viral load and decreases CD4 cell counts in HIV-infected patients with new syphilis infections. AIDS, 2004. 18(15): p. 2075-9.

368. Swaminathan, G., et al., Hepatitis $C$ virus core protein enhances $H I V-1$ replication in human macrophages through TLR2, JNK, and MEK1/2-dependent upregulation of TNF-alpha and IL-6. FEBS Lett, 2014. 588(18): p. 3501-10. 
369. Oyama, T., et al., Expression of cytochrome P450 in tumor tissues and its association with cancer development. Front Biosci, 2004. 9: p. 1967-76.

370. Rodriguez-Antona, C. and M. Ingelman-Sundberg, Cytochrome P450 pharmacogenetics and cancer. Oncogene, 2006. 25(11): p. 1679-91.

371. Hunter, A.L., et al., Cytochrome 450 enzymes and cardiovascular disease. Can J Physiol Pharmacol, 2004. 82(12): p. 1053-60.

372. Stavropoulou, E., G.G. Pircalabioru, and E. Bezirtzoglou, The Role of Cytochromes P450 in Infection. Front Immunol, 2018. 9: p. 89.

373. Holla, V.R., et al., Alterations in the regulation of androgen-sensitive Cyp $4 a$ monooxygenases cause hypertension. Proc Natl Acad Sci U S A, 2001. 98(9): p. 5211-6.

374. Kamata, S., et al., Expression of cytochrome P450 mRNAs in Type II alveolar cells from subjects with chronic obstructive pulmonary disease. Pharmacol Res Perspect, 2018. 6(3): p. e00405.

375. Ha, D., N. Yang, and V. Nadithe, Exosomes as therapeutic drug carriers and delivery vehicles across biological membranes: current perspectives and future challenges. Acta Pharm Sin B, 2016. 6(4): p. 287-96.

376. Yang, T., et al., Exosome delivered anticancer drugs across the blood-brain barrier for brain cancer therapy in Danio rerio. Pharm Res, 2015. 32(6): p. 200314.

377. Alvarez-Erviti, L., et al., Delivery of siRNA to the mouse brain by systemic injection of targeted exosomes. Nat Biotechnol, 2011. 29(4): p. 341-5.

378. Kalani, A. and P. Chaturvedi, Curcumin-primed and curcumin-loaded exosomes: potential neural therapy. Neural Regen Res, 2017. 12(2): p. 205-206.

379. Ivey, N.S., A.G. MacLean, and A.A. Lackner, Acquired immunodeficiency syndrome and the blood-brain barrier. J Neurovirol, 2009. 15(2): p. 111-22.

380. Louboutin, J.P. and D. Strayer, Role of Oxidative Stress in HIV-1-Associated Neurocognitive Disorder and Protection by Gene Delivery of Antioxidant Enzymes. Antioxidants (Basel), 2014. 3(4): p. 770-97.

381. Varatharajan, L. and S.A. Thomas, The transport of anti-HIV drugs across bloodCNS interfaces: summary of current knowledge and recommendations for further research. Antiviral Res, 2009. 82(2): p. A99-109.

382. International Collaboration of Epidemiological Studies of Cervical, C., et al., Carcinoma of the cervix and tobacco smoking: collaborative reanalysis of individual data on 13,541 women with carcinoma of the cervix and 23,017 women without carcinoma of the cervix from 23 epidemiological studies. Int J Cancer, 2006. 118(6): p. 1481-95.

383. Wei, L., et al., Tobacco exposure results in increased E6 and E7 oncogene expression, DNA damage and mutation rates in cells maintaining episomal human papillomavirus 16 genomes. Carcinogenesis, 2014. 35(10): p. 2373-81.

384. Hecht, S.S., Tobacco carcinogens, their biomarkers and tobacco-induced cancer. Nat Rev Cancer, 2003. 3(10): p. 733-44.

385. Alam, S., et al., The cigarette smoke carcinogen benzo[a]pyrene enhances human papillomavirus synthesis. J Virol, 2008. 82(2): p. 1053-8. 
386. Farin, F.M., et al., Expression of cytochrome P450 and microsomal epoxide hydrolase in cervical and oral epithelial cells immortalized by human papillomavirus type 16 E6/E7 genes. Carcinogenesis, 1995. 16(6): p. 1391-401. 


\section{VITA}

Sabina Ranjit, daughter of Krishna P. Ranjitkar and Sanu M. Ranjit, was born in Banepa, Nepal in 1987. She graduated her elementary/Middle school from Vidhya Sagar School, Banepa, Nepal and high school and undergraduate degree in Pharmacy from Kathmandu University, Dhulikhel Nepal. After completion of her undergraduate program, she worked as a quality control officer for 18 months in Omnica Laboratories, a pharmaceutical company in Nepal. She then, joined Southern Illinois University Edwardsville in 2012, where she received her master's degree in Chemistry. Her passion towards science brought her to University of Tennessee Health Science Center, Memphis in 2014 to pursue her doctoral degree in Pharmaceutical Sciences. She will be graduating Dec, 2018. 